\title{
The current-phase relation in Josephson junctions
}

\author{
A. A. Golubov* \\ Faculty of Science and Technology, University of Twente, \\ P. O. Box 217, 7500 AE Enschede, The Netherlands \\ M. Yu. Kupriyanov ${ }^{\dagger}$ \\ Nuclear Physics Institute, Moscow State University, 119992 Moscow, Russia \\ E. Il'ichev ${ }^{\ddagger}$ \\ Department of Cryoelectronics, Institute of Physical High Technology, \\ D-07702 Jena, Germany
}

(Published 26 April 2004)

\begin{abstract}
This review provides a theoretical basis for understanding the current-phase relation (CФR) for the stationary (dc) Josephson effect in various types of superconducting junctions. The authors summarize recent theoretical developments with an emphasis on the fundamental physical mechanisms of the deviations of the CФR from the standard sinusoidal form. A new experimental tool for measuring the CФR is described and its practical applications are discussed. The method allows one to measure the electrical currents in Josephson junctions with a small coupling energy as compared to the thermal energy. A number of examples illustrate the importance of the C $\Phi \mathrm{R}$ measurements for both fundamental physics and applications.
\end{abstract}

\section{CONTENTS}

I. Introduction

II. dc Josephson Effect

A. General properties of the Josephson currentphase relation

B. Basic Josephson structures

III. Josephson Effect in Point Contacts

A. Aslamazov-Larkin model

B. Kulik-Omelyanchuk model, dirty limit (KO-1)

C. Kulik-Omelyanchuk model, clean limit (KO-2)

D. Point contact, the general case

IV. Josephson Junctions with Tunnel-Type Conductivity

A. Ideal tunnel junctions

B. Tunnel junctions with extended barriers

C. Tunneling via localized states

D. SIS structures with high transparency: Depairing by current

V. SNS Junctions

A. The limit of high temperature, $T \approx T_{c}$

1. Weak depairing in the electrodes (rigid boundary conditions)

2. Depairing in superconducting electrodes by the proximity effect

3. Depairing in superconducting electrodes by supercurrent

4. Nonlinear phase shift in the electrodes

5. Influence of the critical temperature of weak-link material on $I_{C} R_{N}$

B. Dirty limit, arbitrary temperature

1. Rigid boundary conditions

a. Structures made from one material $\left(T_{c}\right.$ $=T_{c}^{\prime}$ )

b. SS'S junctions $\left(T_{c} \neq T_{c}^{\prime}\right)$

428

429

\footnotetext{
*Electronic address: A.Golubov@tn.utwente.nl

${ }^{\dagger}$ Electronic address: mkupr@pn.sinp.msu.ru

‡Electronic address: ilichev@ipht-jena.de
}

2. SNS junction at arbitrary $T$ : Depairing due to the proximity effect

3. SS'S junction at arbitrary $T$ : Depairing by the supercurrent

C. Clean SNS junctions

VI. Double-Barrier SINIS Junctions

A. SINIS junctions, clean limi

1. The general case

2. Coherent regime (broad resonances)

3. Incoherent regime (narrow resonances)

B. SINIS junctions, dirty limit

1. The general case

2. Limit of high temperatures

3. Limit of low temperatures

4. Limit of small $\gamma_{\text {eff }}$

5. Limit of large $\gamma_{\text {eff }}$

VII. SFS Josephson Junctions

1. The formalism

2. Complex coherence length

3. Phase variation at the SF interface

B. Current-phase relation in SFS junctions: Simple geometry

1. $0-\pi$ transitions due to oscillating order parameter

2. $0-\pi$ transitions due to phase jumps at the $\mathrm{SF}$ interfaces

F. Double-barrier SIFIS junction

D. Measurements of small critical currents 
F. Current-phase relation in hybrid S-2DEG-S junctions

G. Current-phase relation anomalies in high- $T_{c}$ superconducting junctions

1. $d$-wave effects in high- $T_{c}$ superconductors

2. Asymmetric $45^{\circ}$ grain-boundary junction

3. Symmetric $45^{\circ}$ grain-boundary junction: Manifestation of midgap states in the current-phase relation

4. $c$-axis high- $T_{c}$ superconducting junctions

5. General remarks

IX. Summary and Outlook

Acknowledgments

Appendix: Microscopic Theory of Superconductivity

1. Green's functions

2. The quasiclassical approximation

a. Eilenberger equations

b. The dirty limit: Usadel equations

c. $\Phi$ parametrization

3. Ginzburg-Landau equations

4. Critical current in a diffusive point contact: Derivation of the KO-1 result

References

\section{INTRODUCTION}

The Josephson effect was discovered by Brian Josephson in 1962 (see also Josephson, 1964, 1965). The stationary Josephson effect was first observed experimentally by Anderson and Rowell (1963) and Rowell (1963), and the nonstationary Josephson effect was observed by Yanson et al. (1965). Since that time, there has been a continuously growing interest in the fundamental physics and applications of this effect. The achievements in Josephson-junction technology have made it possible to develop a variety of sensors for detecting ultralow magnetic fields and weak electromagnetic radiation; they have also enabled the fabrication, testing, and application of ultrafast digital rapid single flux quantum (RSFQ) circuits as well as the design of large-scale integrated circuits for signal processing and general purpose computing (Likharev and Semenov, 1991; Likharev, 1996, 2000; SEMATECH, 2001). In the present literature, there is no recent overview of this area of physics available to both experts and people entering the field.

Classical developments are summarized in the review by Likharev (1979) and several standard textbooks that deal with the Josephson effect (Barone and Paterno, 1982; Likharev, 1986; Schmidt, 1997), where thorough treatments are provided of the basic phenomena of the Josephson effect in tunnel junctions and weak links.

Several recent review articles have been devoted to applications of the Josephson effect, e.g., in high- $T_{c}$ superconductors and Josephson quantum bits. ${ }^{1}$ At the same time, recent progress in the physics of the Joseph-

\footnotetext{
${ }^{1}$ See, for example, Kupriyanov and Likharev, 1990; Braginski, 1992; Sigrist and Rice, 1995; van Harlingen, 1995; Delin and Kleinsasser, 1996; Kashiwaya and Tanaka, 2000; Tsuei and Kirtley, 2000; Löfwander et al., 2001; Makhlin et al., 2001; Hilgenkamp and Mannhart, 2002.
}

son effect justifies an overview of the fundamentals of the Josephson effect on a more general level.

The purpose of the present review is to provide a theoretical basis for the dependence of the supercurrent $I_{S}$ on the phase difference $\varphi$ and to discuss the forms this dependence takes in Josephson junctions of different types: superconductor-normal-superconductor (SNS), superconductor-insulator-superconductor (SIS), doublebarrier (SINIS), superconductor-ferromagnet-superconductor (SFS), and superconductor two-dimensional electron gas superconductor (S-2DEG-S) junctions, and superconductor-constriction-superconductor ( $\mathrm{ScS}$ ) point contacts. Unconventional symmetry in the order parameter of a high- $T_{c}$ superconductor, as manifested in the current-phase relation (CФR) will also be discussed.

Recently, a new experimental tool has been developed (Rifkin and Deaver, 1976; Il'ichev, Zakosarenko, IJsselstein, et al., 1998a; Il'ichev, Zakosarenko, Schultze, et al., 2000) and applied to the study of the C $\Phi R$ for a variety of Josephson junctions. We shall describe this method and its practical applications. It is important that the method has a resolution permitting the study of weak links with Josephson energies smaller than the thermal energy. We shall illustrate by a number of examples the importance of these experimental studies for fundamental physics and applications. The examples include measurements of small critical currents in Josephson junctions, a calibration of second oxidation in SINIS junctions, and studies of hybrid S-2DEG-S Josephson devices, CФR anomalies in high- $T_{c}$ superconducting junctions, physics of $\pi$ states in SFS junctions and qubit structures.

The main emphasis in this review is on the general nature of the Josephson effect and on fundamental physical mechanisms that control the CФR. At the same time, some details are provided regarding types of weak links and their fabrication. References to original experimental papers are given in the appropriate places.

\section{II. dc JOSEPHSON EFFECT}

Josephson (1962) predicted that a supercurrent $I_{S}$ could exist between two superconductors separated by a thin insulating layer and that its value would be proportional to the sine of the difference $\varphi=\chi_{1}-\chi_{2}$ of the phases of the superconductor order parameters $\Delta_{1} \exp \left\{i \chi_{1}\right\}$ and $\Delta_{2} \exp \left\{i \chi_{2}\right\}$,

$$
I_{S}(\varphi)=I_{C} \sin \varphi
$$

the so-called $d c$ Josephson effect. The maximum current $I_{C}$ in that CФR is the critical current. Further studies have shown (de Gennes, 1966; for a review see Likharev, 1979) that the effect extends beyond Josephson's predictions and can exist if superconductors are connected by a "weak link" of any physical nature (normal metal, semiconductor, superconductor with smaller critical temperature, geometrical constriction, etc.)

The CФR is an important characteristic of a Josephson junction. In only a few cases does the CФR reduce 


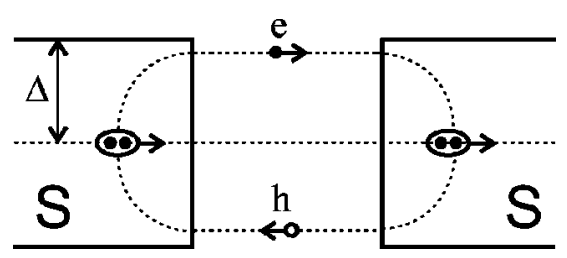

FIG. 1. Formation of Andreev levels in a Josephson junction. An electron $e$ and the Andreev-reflected hole $h$ are shown. A pair of correlated electrons is transferred from the left superconductor to the right one, creating a supercurrent flow across a junction.

to the familiar sinusoidal form of Eq. (1), which is ordinarily used to study the dynamics and ultimate performance of analogous and digital devices based on Josephson junctions (see van Duzer and Turner, 1981; Barone and Paterno, 1982; Likharev, 1986; Gallop, 1991; Orlando and Delin, 1991; Tinkham, 1996; Schmidt, 1997; Kadin, 1999).

The physics of the dc Josephson effect can be understood if we take into account that a quasiparticle located in the weak link cannot penetrate directly into a superconductor if its energy is smaller than the superconducting energy gap. However, another form of charge transport, the so-called Andreev reflection (Andreev, 1964), can occur. An electron with momentum $k$ impinging on one of the interfaces is converted into a hole moving in the opposite direction, thus generating a Cooper pair in a superconductor. This hole is consequently Andreev reflected at the second interface and is converted back to an electron, leading to the destruction of a Cooper pair (see Fig. 1). As a result of this cycle, a pair of correlated electrons is transferred from one superconductor to another, creating a supercurrent flow across a junction. Since Andreev reflection amplitudes depend on the corresponding phases $\chi_{1,2}$, the resulting current depends on the phase difference $\varphi$, thus leading to the dc Josephson effect.

Due to the electron-hole interference in the quantum well, formed by the pairing potentials of the superconducting electrodes, standing waves with quantized energy $E_{A B}$ appear in the weak-link region The corresponding quantum states are referred to as Andreev bound states. The physics of Andreev bound states in Josephson junctions has been studied extensively, starting with the pioneering work of Kulik (1969).

It follows from the microscopic theory of superconductivity (see review by Lambert and Raimondi, 1998; Belzig et al., 1999; Kopnin, 2001) that in stationary situations the supercurrent across a Josephson junction,

$$
I_{S}(\varphi) \propto \int_{-\infty}^{\infty} d E[1-2 f(E)] \operatorname{Im}\left\{I_{E}(\varphi)\right\},
$$

depends on the electron energy distribution function $f(E)$ and the spectral current $\operatorname{Im}\left\{I_{E}\right\}$. The spectral current incorporates information on the energy distribution of the Andreev bound state in a junction. $\operatorname{Im}\left\{I_{E}\right\}$ depends on the distance $d$, between the superconductors and the transport parameters of the junction's materials (resistivities $\rho_{1,2}$, Fermi velocities $v_{F 1,2}$, and interface parameters).

In structures where the momentum of an electron in the weak-link region is a good quantum number (socalled "clean" Josephson junctions), Andreev bound states form a regular structure in energy and $\operatorname{Im}\left\{I_{E}(\varphi)\right\}$ is peaked at the corresponding energies (Kulik, 1969; Ishii, 1970; Bardeen and Johnson, 1972; Bagwell, 1992; Schüssler and Kummel, 1993; Tang, Wang, and Zhang, 1996; Tang, Wang, and Zhu, 1996).

An increase of the degree of disorder in the weak link leads to a broadening and decrease of the amplitudes of the peaks. The disorder generates a distribution of the lengths of the electron trajectories in the weak link. Therefore $\operatorname{Im}\left\{I_{E}(\varphi)\right\}$ for a disordered junction is a weighted average of the ballistic result over the corresponding distribution. This makes the spectral current $\operatorname{Im}\left\{I_{E}(\varphi)\right\}$ a continuous function of energy. Other sources of the broadening of Andreev bound states are the Doppler shifts of Andreev bound-state energies due to current flow (see, for example, Fogelström et al., 1997) and many-body lifetime effects (Freericks et al., 2002).

The electron energy distribution function in Eq. (2) defines the population of Andreev bound states at a given temperature. Thus Eq. (2) shows that the whole supercurrent $I_{S}(\varphi)$ is the sum of the partial currents transported via Andreev bound states. Therefore one can modify the shape of $I_{S}(\varphi)$ in two ways: (a) by modifying the spectral current $\operatorname{Im}\left\{I_{E}(\varphi)\right\}$, changing the material parameters or the geometry of a junction; (b) by manipulating the occupation numbers of Andreev bound states, i.e., creating a nonequilibrium distribution function $f(E)$ in a weak link.

Nonequilibrium effects in weak links have been intensively studied by many authors. ${ }^{2}$ These effects occur either in the nonstationary regime, when $I>I_{C}$ and a voltage is generated across the junction (stimulation by current), under microwave irradiation, or in multiterminal structures, when the junction is in a stationary regime but voltage is applied via additional terminals.

In this review we shall restrict ourselves to the stationary Josephson effect in two-terminal weak links when nonequilibrium effects do not play a role. This corresponds to the above-mentioned case (a), when the CФR is determined by the spectral current $\operatorname{Im}\left\{I_{E}(\varphi)\right\}$, depend-

\footnotetext{
${ }^{2}$ See, for example, Zaitsev, 1976, 1993, 1995; Artemenko et al., 1979; Zaikin and Zharkov, 1981; Aslamazov and Lempitskii, 1982; Lempitskii, 1983; Zaikin 1983, 1988; Aslamazov and Volkov, 1986; van Wees et al., 1991; Shumeiko et al., 1993; Gorelik et al., 1995; Volkov, 1995; Chang and Bagwell, 1997; Samuelsson et al., 1997, 2000, 2001; Volkov and Takayanagi, 1997; Morpurgo et al., 1998; Tolga Ilhan et al., 1998; Wilhelm et al., 1998, 2000; Yip, 1998; Baselmans et al., 1999, 2001; Kutchinsky et al., 1999, 2000; Neurohr et al., 1999; Heikkilä et al., 2000, 2002; Seviour and Volkov, 2000; Sun et al., 2000; Baselmans, Heikkila, et al. 2002; Baselmans, van Wees, et al., 2002; Huang et al., 2002; Zhu et al., 2002; Brinkman et al., 2003.
} 
ing on material parameters, junction geometry, and current flow.

\section{A. General properties of the Josephson current-phase relation}

There are several properties of the C $\Phi R$ that are rather general and depend neither on the junction's materials and geometry nor on the theoretical model used to describe the processes in the junction.

(1) A change of phase of the order parameter of $2 \pi$ in any of the electrodes is not accompanied by a change in their physical state. Consequently this change must not influence the supercurrent across a junction, and $I_{S}(\varphi)$ should be a $2 \pi$ periodic function,

$$
I_{S}(\varphi)=I_{S}(\varphi+2 \pi) \text {. }
$$

(2) Changing the direction of a supercurrent flow across the junction must cause a change of the sign of the phase difference; therefore

$$
I_{S}(\varphi)=-I_{S}(-\varphi) \text {. }
$$

Note that Eq. (4) is violated in superconductors with broken time-reversal symmetry, leading to spontaneous currents. These effects have been discussed for Josephson junctions between superconductors with unconventional pairing symmetry (Geshkenbein et al., 1986, 1987; Yip, 1995; Fogelstrom et al., 1997; Tanaka and Kashiwaya, 1997b; Sigrist, 1998) and for superconductor-ferromagnet-superconductor (SFS) junctions (Krawiec et al., 2002). Discussion of spontaneous currents and their mechanisms is beyond the scope of this review and Eq. (4) is fulfilled in all considered cases.

(3) A dc supercurrent can flow only if there is a gradient of the order-parameter phase. Hence, in the absence of phase difference, $\varphi=0$, there should be zero supercurrent,

$I_{S}(2 \pi n)=0, \quad n=0, \pm 1, \pm 2, \ldots$

(4) It follows from (1) and (2) that the supercurrent should also be zero at $\varphi=\pi n$,

$I_{S}(\pi n)=0, \quad n=0, \pm 1, \pm 2, \ldots$;

therefore it is sufficient to consider the $I_{S}(\varphi)$ only in the interval $0<\varphi<\pi$.

As follows from Eqs. (1)-(4), $I_{S}(\varphi)$ can in general be decomposed into a Fourier series (see, for example, Tanaka and Kashiwaya, 1997b)

$$
I_{S}(\varphi)=\sum_{n \geqslant 1}\left\{I_{n} \sin (n \varphi)+J_{n} \cos (n \varphi)\right\},
$$

where $I_{n}$ and $J_{n}$ are coefficients to be determined. The $J_{n}$ vanish if time-reversal symmetry is not broken.

The free energy $E_{J}$ of a Josephson junction is generally given by an integral $(\hbar / 2 e) \int_{0}^{\varphi} I_{S}(\chi) d \chi$. If the CФR

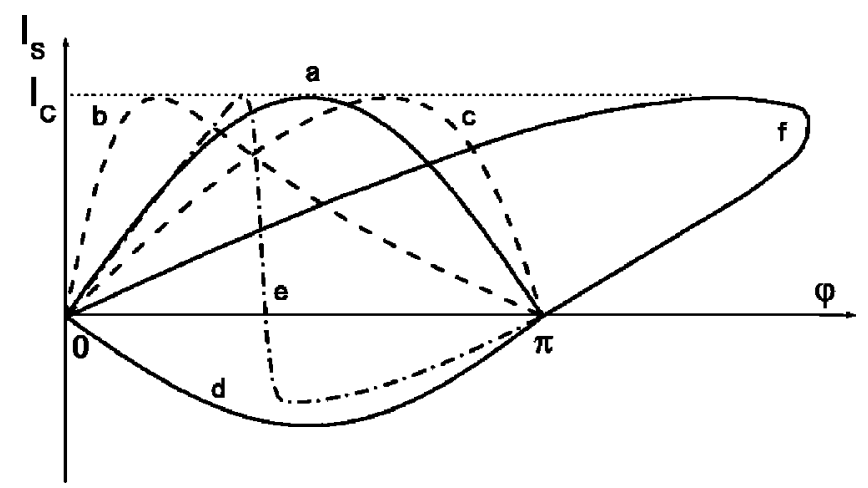

FIG. 2. Various types of current-phase relation (CФR): curve $a$, the standard sinusoidal $\mathrm{C} \Phi \mathrm{R}$; curves $b$ and $c$, deviations from the standard CФR, when the critical current is achieved at $\varphi_{\max } \leqslant \pi / 2$ and $\varphi_{\max } \geqslant \pi / 2$, respectively; curve $d$, a $\pi$ junction: curve $e$ has the CФR whose energy-phase relation has two minima at $\varphi=0$ and $\varphi=\pi$; curve $f$, multivalued $\mathrm{C} \Phi \mathrm{R}$ that does not correspond to a true Josephson effect and may have various causes (see the text).

is sinusoidal, $I_{S}(\varphi)=I_{C} \sin \varphi$ (Fig. 2, curve $a$ ), the dependence of $E_{J}$ on $\varphi$ has the standard form

$$
E_{J}(\varphi)=\frac{\hbar I_{C}}{2 e}(1-\cos \varphi)
$$

also well known as the $2 \pi$-periodic "washboard potential." The standard junction has an energy minimum at $\varphi=0$ when there is no current flowing across the junction. For junctions with a small capacitance, the charging energy becomes important and should be added to $E_{J}(\varphi)$ (Likharev, 1986; Tinkham, 1996).

Possible types of CФR in Josephson junctions are shown in Fig. 2. Curve $a$ is the standard sinusoidal CФR. The critical current $I_{C}=\max \left[I_{S}(\varphi)\right]$ can be achieved at both $\varphi_{\max } \leqslant \pi / 2$ and $\varphi_{\max } \geqslant \pi / 2$ (the curves $b$ and $c$, respectively).

An interesting special case is the junction with $I_{C}<0$ (Fig. 2, curve $d$ ), the so-called $\pi$ junction (Bulaevskii et al., 1977). According to Eq. (8), such a junction has an energy minimum at $\varphi=\pi$, i.e., it provides a phase shift of $\pi$ in the ground state.

A $\pi$ junction may be used as the phase inverter in superconducting digital circuits. The so-called complementary Josephson digital devices (e.g., the $\pi$ SQUID), were discussed by Terzioglu and Beasley (1998). Ioffe et al. (1999), Blais and Zagoskin (2000), and Zagoskin (2002) proposed $\pi$ junctions as candidates for engineering a quantum two-level system, or qubit, which is the basic element of a quantum computer. Blatter et al. (2001) suggested structures including arrays of conventional ( 0 ) and $\pi$ junctions for the realization of a "quiet" phase qubit. A topologically stable qubit, based on a triangular or more complicated $\pi$ junction array, was discussed by Blatter et al. (2001) as well.

In some special cases $I_{S}(\varphi)$ may cross the horizontal axis at a position in between $\varphi=0$ and $\varphi=\pi$, as shown in Fig. 2, curve $e$. The energy-phase relation in this case has two minima at $\varphi=0$ and $\varphi=\pi$. 
One might consider the possibility of a multivalued CФR with $I_{S}(\pi n) \neq 0$, as shown in Fig. 2, curve $f$. However, structures with a multivalued CФR cannot be regarded as real Josephson junctions. Such a currentphase relation may be realized either due to Abrikosov vortices (see Kupriyanov et al., 1975; Likharev, 1979) or phase-slip centers inside the junction (Ivlev and Kopnin, 1984; Martin-Rodero et al., 1994; Sols and Ferrer, 1994). The CФR in this case is controlled by completely different physical processes than in the standard Josephson junctions.

$I_{S}(\varphi)$ dependences of the type shown in Fig. 2 by curves $b$ and $c$ may be roughly described by the expression (see Likharev, 1976, 1979; Zubkov et al., 1981; Schüssler and Kümmel, 1993)

$$
I_{£}(\varphi)=I_{C} \sin \left[\varphi-£ I_{£}(\varphi)\right],
$$

by which a weak link is represented as an ideal Josephson junction with $I_{S}(\varphi) \propto \sin \varphi$ connected in series with a nonlinear inductance $£$. This inductance can be either positive (Fig. 2, curve $b$ ) or negative (Fig. 2, curve $c$ ) and is a consequence of the specific properties of a superconducting condensate. The presentation of the C $\Phi R$ in the form of Eq. (9) may be useful for the analysis of a mode of operation of a system containing several Josephson junctions with a nonsinusoidal CФR.

\section{B. Basic Josephson structures}

The Josephson effect may be observed in a variety of structures. To realize such structures it is enough to fabricate a "weak" place interrupting the supercurrent flow in a superconductor or suppress the ability of a superconductor to carry a current, e.g., by deposition of a normal metal on its top, by implantation of impurities within a restricted volume, or by changing the geometry of a sample. All of these possibilities have been extensively discussed in reviews and textbooks (see Likharev, 1979, 1986; van Duzer and Turner, 1981; Barone and Paterno, 1982; Gallop, 1991; Orlando and Delin, 1991; Tinkham, 1996; Schmidt, 1997; Kadin, 1999). Among them only a few configurations have importance for practical applications. These are point contacts, tunnel junctions, sandwiches, and variable-thickness bridges (having a normal metal, a semiconductor, or a weak ferromagnet as a weak-link material), and double-barrier structures.

We shall begin our analysis by considering the dc Josephson effect in point contacts in Sec. III. The point contact is a structure with strong supercurrent concentration and provides the simplest system in which the Josephson effect may be observed. A point contact may be fabricated by placing a superconducting tip on top of a bulk superconductor or by depositing a superconductor on top of a superconductor-dielectric bilayer with a submicrometer hole in the insulator which defines a small contact area. Contacts based on a two-dimensional electron gas are also close to this type of structure. Point contacts are well-defined systems in which the fundamental physics of the Josephson effect can be most eas- ily studied theoretically and verified experimentally, because all nonlinear, nonstationary, and nonequilibrium processes are localized within the weak link, while the electrodes may be considered as undisturbed (i.e., in equilibrium).

Fabrication and measurements of ultrasmall superconducting point contacts, in which the constriction size is reduced towards atomic dimensions, was reported by several groups in atomic break junctions and nanotubes (Muller et al., 1994; van der Post et al., 1994; Vleeming et al., 1994; Scheer et al., 1997, 2001; Ludoph et al., 2000; Buitelaar et al., 2002, 2003; Agrait et al., 2003). Such atomic contacts have proven a rich test bed for concepts from mesoscopic physics like multiple Andreev reflection, shot noise, conductance quantization and fluctuations, and dynamical Coulomb blockade.

In tunnel junctions, discussed in Sec. IV, the weak place is formed by a dielectric layer separating two superconducting electrodes. An ideal tunnel junction is characterized by a sinusoidal CФR. In many real tunnel junctions, such as those based on $\mathrm{NbAl} / \mathrm{AlO}_{x} / \mathrm{Nb}$ technology (Gurvitch et al., 1983), the base electrode has the form of a NbAl bilayer. The proximity effect may strongly influence the supercurrent in such junctions, while the CФR remains sinusoidal (see Golubov, Kupriyanov, and Lukichev, 1984; Golubov and Kupriyanov 1988, 1989; Golubov, Gurvitch, et al., 1993; Golubov, Houwman, et al., 1995). $\mathrm{AlO}_{x}$ forms a thin dielectric layer with a high potential barrier having an approximately trapezoidal barrier profile (Tolpygo, Cimpoiasu, et al., 2003). In order to increase the current density up to the values of $100-200 \mathrm{kA} / \mathrm{cm}^{2}$, required for fabrication of high- $J_{C}$, intrinsically shunted tunnel junctions (where $J_{C}$ is the critical current density), this barrier can be made extremely thin, of the order of a few atomic layers, by decreasing the degree of $\mathrm{Al}$ oxidation to a level at which the formation of a disordered region is expected rather than a high-quality $\mathrm{AlO}_{x}$ barrier. The properties of these junctions should be close to those of superconductor-correlated metal-superconductor structures studied theoretically by Nikolić et al. (2001) and Freericks et al. (2002). The transport properties of high$J_{C}$ tunnel junctions were analyzed by Naveh et al. (2000) and Rippard et al. (2002), who have shown that electronic transport across these structures may be dominated by resonant tunneling via localized states in the disordered region.

High-quality Nb-based tunnel junctions have also been fabricated with $\mathrm{AlN}_{x}$ barriers (see Iosad et al., 2003; Lapitskaya et al., 2003). In tunnel structures having $\mathrm{NbN}$ electrodes, either $\mathrm{TiN}_{x}$ (see, for example, Ishizaki et al., 2003; Takeda et al., 2003; Uzawa et al., 2003; Wang, Saito, et al., 2003) or $\mathrm{MgO}$ (see, for example, Johnson et al., 2003) barriers have been successfully used for fabrication of SIS mixers and RSFQ circuits.

There exists another type of tunnel junction consisting of homogeneous electrodes divided by a thick and relatively low potential barrier (as in high- $T_{c}$ junctions with PrBaCuO barriers; see, e.g., Gao et al., 1990). The situation in structures with extended barriers may be more 
complex due to the appearance of additional Andreev bound states (see Wendin and Shumeiko, 1996a, 1996b) or localized states inside the barrier. ${ }^{3}$ These effects as well as the influence of depairing processes in the electrodes on the shape of the CФR will be discussed in Sec. IV.

In Sec. V, SNS sandwiches and variable-thickness bridges are discussed. In these junctions the weak link is formed by a normal metal separating two superconducting electrodes. In the last few years, interest in SNS weak links has grown continuously. ${ }^{4}$ They have been considered as a reliable elementary base for the realization of a programmable voltage standard (see Benz and Burroughs, 1997; Hamilton et al., 1997; Hagedorn et al., 2001, 2002; Jeanneret et al., 2001; Dresselhaus et al., 2003) and have also been used for fabrication of smallscale RSFQ circuits (see van Duzer et al., 2002; Hagedorn et al., 2003).

In these junctions the shape of $I_{S}(\varphi)$ not only depends on temperature and the distance between electrodes, but also on the critical temperature of the interlayer and the transport parameters of both metals and the interfaces. As we shall see in Sec. V, one may expect in these junctions the realizations of the C $\Phi R$ presented in Fig. 2.

An increased concentration of localized states inside the barrier or a decrease of the barrier thickness under a fixed localized state concentration may lead, as discussed above, to the formation of superconductorcorrelated metal-superconductor structures, in which current flows via a material that is close to the metalinsulator transition. Structures of that kind were fabricated by Kaul et al. (2001), who used $\mathrm{TaN}_{x}$ as a barrier material sandwiched between superconducting $\mathrm{NbN}$ electrodes. $\mathrm{TaN}_{x}$ was identified as a promising barrier material due to tunability of its resistivity over a wide range by varying growth conditions (Willmott, 1972). The Ta-N system exhibits a remarkable variety of equilibrium and metastable phases. More than 11 have been reported, while very little is known about their relative stability (see Shin et al., 2002; Stampfl and Freeman, 2003). Stampfl and Freeman (2003) have proposed that $\mathrm{Ta}$ vacancies and/or regions of Ta-deficient structures are primarily responsible for the metal-insulator transition observed experimentally in $\mathrm{TaN}_{x}$ at $x \approx 0.6$. Proper-

\footnotetext{
${ }^{3}$ See Gubankov et al., 1985; Amatuni et al., 1987; Xu et al., 1990; Golubov et al., 1994; Vengrus et al., 1994; Dömmel et al., 1995; Kupriyanov and Tsai, 1995; Satoh et al., 1995, 1998; Wen et al., 1995; Verhoeven et al., 1996; Gerritsma et al., 1997; Horstmann et al., 1997, 1998; Verbist et al., 1997; Yoshida et al., 1997; Sun and Gao, 2000; Yoshida, 2000.

${ }^{4}$ See, for example, Imamura and Hasio, 1989; Benz, 1995; Benz and Burroughs, 1997; Sachse et al., 1997; Fritzsch et al., 1998, 1999; Whiteley et al., 1998; Lacquaniti et al., 1999, 2000, 2001, 2003; Niemeyer, 2000; Pöpel, Hagedorn, Buchholz, et al., 2000; Hadfield, Burnell, Booij, et al., 2001; Hadfield, Burnell, Kang, et al.. 2001; Hagedorn et al., 2001, 2002; Kaul and van Duzer, 2001; Ohta et al., 2001; Schubert et al., 2001; van Duzer et al., 2002.
}

ties of a Josephson junction with the barrier tuned through a metal-insulator transition were described theoretically by Freericks et al. (2001, 2002, 2003).

Section VI deals with SINIS double-barrier junctions. Here the weak link is formed by a normal metal separated by tunnel barriers from each of the superconducting electrodes ( $\mathrm{N}$ could also be a weak superconductor, $\mathrm{S}^{\prime}$, e.g., $\mathrm{Al}$ ). The barriers may be naturally formed at the NS interfaces during the fabrication or made artificially. Double-barrier junctions are unique devices, which combine the main advantages of weak links and tunnel junctions (see Kupriyanov and Lukichev, 1988a, 1988b). It is important to note that these structures can be fabricated, making use of a modification of the standard SIS junction fabrication process (Gurvitch et al., 1983). Their parameters are intrinsically homogeneous due to additional averaging of the irregularities of the interface transparency by the intermediate Al layer, which is characterized by a large coherence length. They are reproducible on the on-chip level. This fact has been successfully demonstrated by several technological groups. ${ }^{5}$ SINIS junctions are now considered prospective elements for superconducting electronics. We shall show below in Sec. VI that the shape of $I_{S}(\varphi)$ in these junctions strongly depends on the critical temperature of the $S^{\prime}$ material.

At relatively small critical current density $\left(\leqslant 1 \mathrm{kA} / \mathrm{cm}^{2}\right)$ SINIS structures can be fabricated with good reproducibility of their parameters. This has been demonstrated by Wender et al. (2003) and by Schulze et al. (2000), who fabricated, respectively, a 1-V 14-bit programmable voltage standard containing 8192 SINIS junctions and a $10-\mathrm{V}$ standard based on more than 70000 junctions. Practical realization of double-barrier junctions with high critical current density at the level of $10 \mathrm{kA} / \mathrm{cm}^{2}$ has faced difficulties in performing the second oxidation in a controllable way. Due to the different morphology of the first and second $\mathrm{Al}$ films in a $\mathrm{Nb} / \mathrm{Al} / \mathrm{AlO}_{x} / \mathrm{Al} / \mathrm{AlO}_{x} / \mathrm{Nb}$ structure, the same oxidation conditions result in increasing barrier asymmetry with decreasing interlayer thickness and oxygen exposure (see Cassel et al., 2001; Born et al., 2003; Tolpygo, Brinkman, et al., 2003). One possible technological solution of this problem, using $\mathrm{AuAl}_{2}$ instead of the second $\mathrm{Al}$ film, was suggested by Wang, Chang, et al. (2003).

In SFS Josephson junctions, discussed in Sec. VII, the weak link is formed by a ferromagnetic metal placed

\footnotetext{
${ }^{5}$ See Amin et al., 1992; Nevirkovets, 1995, 1997; Capogna and Blamire, 1996; Nevirkovets et al., 1996, 1999, 2000; Capogna et al., 1997; Maezawa and Shoji, 1997; Sugiyama et al., 1997, 1999; Balashov et al., 1998, 1999, 2000, 2001; Schulze et al., 1998, 2000; Behr et al., 1999; Buchholz and Kessel, 1999; Brinkman et al., 1999, 2001; Khabipov et al., 1999, 2002a, 2002b; Kupriyanov et al., 1999; Buchholz et al., 2001; Cassel et al., 2001; Kohlmann et al., 2001, 2002; Nevirkovets, Ketterson, and Rowell, 2001; Nevirkovets, Ketterson, and Siegel, 2001; Shaternik et al., 2001; Kieler et al., 2002; Nevirkovets and Ketterson, 2002; Yamamori et al., 2002; Tolpygo, Brinkman, et al., 2003.
} 
between the $\mathrm{S}$ electrodes. Until now, only a planar geometry has been realized in experiments by Ryazanov et al. (2000, 2001a, 2001b, 2002), Blum et al. (2002), Kontos et al. (2002), and Surgers et al. (2002). In these experiments the transition from the 0 state (curve $a$ Fig. 2) to the $\pi$ state (curve $d$ Fig. 2) was observed experimentally. Theory predicts that if the weak link in a Josephson junction is formed by a magnetic material, either metal or dielectric, the shape of the CФR may have the variety of forms shown in Fig. 2.

In Sec. VIII we discuss the experimental method for measuring the CФR and its applications. The range of validity of the method, measuring technique, and calibration are discussed. The applications include measurements of small critical current, the CФR in SINIS, S/2DEG/S, and high- $T_{c}$ superconducting junctions. Of particular interest are the CФR anomalies observed in high- $T_{c}$ junctions. High- $T_{c}$ superconductors are characterized by an unconventional $d$-wave symmetry of the order parameter as was proved by phase sensitive tests [see review papers by van Harlingen (1995) and by Tsuei and Kirtley (2000)]. Since the theory of the Josephson effect between $d$-wave superconductors has been extensively discussed in the review papers by Kashiwaya and Tanaka (2000) and Löfwander et al. (2001), we shall introduce the relevant theoretical concepts only in connection with the measurements in Sec. VIII.

The theoretical results discussed in this review have been obtained within the quasiclassical theory. This theory has proved to be very successful in describing superconductivity and the properties of weak links in conventional materials. On the other hand, some material-specific aspects, in particular correlation and self-energy effects in high- $T_{c}$ superconducting oxides and in materials close to metal-insulator transition, are outside the scope of the quasiclassical theory. The influence of these effects on the properties of Josephson junctions was discussed by Martin-Rodero et al. (1994, 1999), Levy Yeyati et al. (1995), Cuevas et al. (1996), Martin and Annett (1998, 1999), Nikolić et al. (2001, 2002), and Freericks et al. (2002).

\section{JOSEPHSON EFFECT IN POINT CONTACTS}

Point contacts are structures with strong supercurrent concentration. If the constriction size is smaller than the electronic mean free path, the contact is usually called a Sharvin point contact (Sharvin, 1965). Upon further reduction of the transverse size, making it comparable with the Fermi wavelength, the contact enters the quantum regime, in which conductance is quantized. This regime was realized in normally conducting structures with two-dimensional electron gas by van Wees et al. (1988) and in superconducting structures by Takayanagi and Kawakami (1985) and Takayanagi et al. (1995a, 1995b) (see the recent review by Schäpers, 2001). Another regime is that of the diffusive point contact, with a size larger than the electronic mean free path (the so-called Maxwell contact).
Superconducting constrictions serve as model systems for understanding the fundamental physics of the Josephson effect. In this section we discuss the classical models of Aslamazov and Larkin and Kulik and Omelyanchuk, their extensions, and the physics of Andreev bound states.

\section{A. Aslamazov-Larkin model}

The model suggested by Aslamazov and Larkin (1969) illustrates the basic principle of Josephson coupling: the coupling occurs due to an overlap of superconducting order parameters from both electrodes in the weak-link region. The model is applicable near the $T_{c}$ of the electrodes if the characteristic size of a weak link is sufficiently small, $L_{\text {eff }} \ll \xi(T)$, where $\xi(T)$ is the Ginzburg-Landau coherence length [see Eq. (A29)]. The details of the derivation for the supercurrent can be found in the review by Likharev (1979). The result is (Aslamazov and Larkin, 1969)

$$
I_{S}(\varphi)=\frac{\pi \Delta_{1} \Delta_{2}}{4 e R_{N} T} \sin \varphi,
$$

where $\Delta_{1,2}$ are the magnitudes of the pair potentials in the superconducting electrodes and $R_{N}$ is the normalstate resistance of the weak link. We write the Boltzmann constant as $k_{B}=1$ in Eq. (10) and elsewhere, unless stated otherwise.

Equation (10) is very general: it does not depend on the electronic mean free path in the weak link and is applicable to all types of weak links near $T_{c}$. According to Eq. (10), near $T_{c}$ the CФR is always sinusoidal, independent of the material of the weak link, which is characterized only by its resistance $R_{N}$.

The Aslamazov-Larkin model can be generalized to arbitrary temperatures in both the clean and dirty limits. At low temperature the C $\mathrm{C} \mathrm{R}$ is no longer universal, but depends on the electronic mean free path in the weak link.

\section{B. Kulik-Omelyanchuk model, dirty limit (KO-1)}

Kulik and Omelyanchuk (1975a) considered a point contact as a diffusive quasi-one-dimensional wire, connecting two superconductors, having length $d \ll \sqrt{\xi_{0} l}$ and transverse size $a \ll d$, where $\xi_{0}=\hbar v_{F} / 2 \pi T_{c}$ and $l \ll \xi_{0}$ is the electronic mean free path. In this case, the supercurrent can be calculated from the Usadel equations (A11)-(A14), in which nongradient terms are neglected (see the derivation in Appendix, Sec. 2). For a symmetric junction formed by identical superconductors $\left(\Delta_{1}\right.$ $=\Delta_{2}=\Delta$ ) the result is

$$
I_{S}(\varphi)=\frac{4 \pi T}{e R_{N}} \sum_{\omega>0} \frac{\Delta \cos (\varphi / 2)}{\delta} \arctan \frac{\Delta \sin (\varphi / 2)}{\delta},
$$

where $\delta=\sqrt{\Delta^{2} \cos ^{2}(\varphi / 2)+\omega^{2}}$ and $\omega=\pi T(2 n+1)$ are the Matsubara frequency. The C $\Phi \mathrm{R}$ at arbitrary temperature can be calculated numerically from Eq. (11). Examples of CФR's for this model, hereafter referred to 


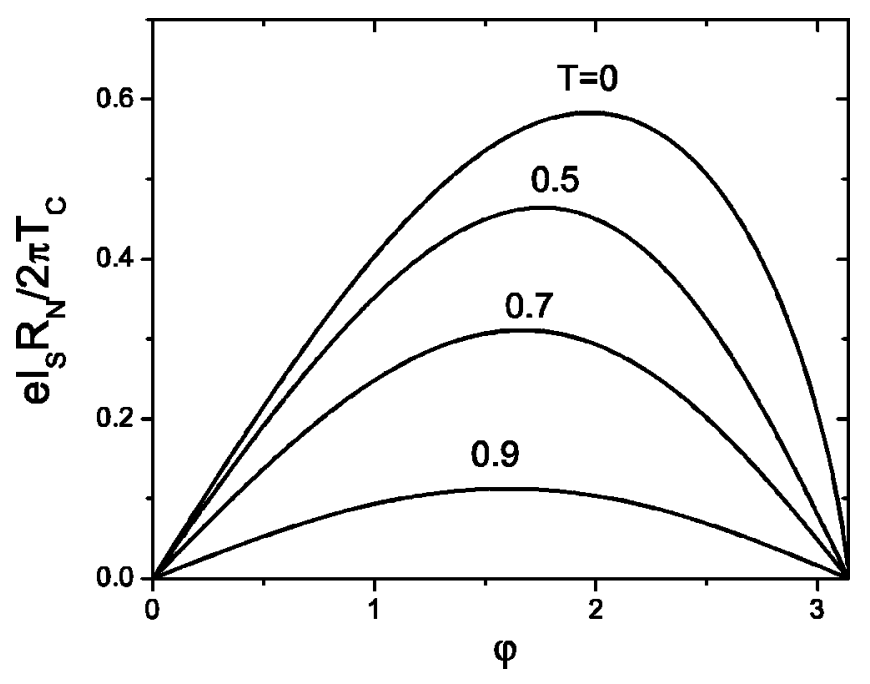

FIG. 3. Current-phase relations for a symmetric diffusive point contact - the Kulik-Omelyanchuk (KO-1) model-at various temperatures $T$, normalized to $T_{c}$.

as the KO-1 model, are shown in Fig. 3. The curves $I_{S}(\varphi)$ are nonsinusoidal at low temperatures and reduce to the Aslamazov-Larkin result for $T \rightarrow T_{c}$.

Equation (11) was generalized to the asymmetric case $\left(\Delta_{1} \neq \Delta_{2}\right)$ by Zubkov et al. (1981; see Appendix, Sec. 2). As the ratio $\Delta_{2} / \Delta_{1}$ increases, the CФR becomes closer to sinusoidal. For $\Delta_{2} / \Delta_{1} \gg 1$ the result is

$$
I_{S}(\varphi)=\frac{\Delta_{1}}{e R_{N}}\left[\ln \frac{2 \Delta_{2}}{\Delta_{1}[1+\cos (\varphi)]}\right] \sin \varphi
$$

i.e., there is a slow logarithmic crossover to $\sin \varphi$ dependence.

\section{Kulik-Omelyanchuk model, clean limit (KO-2)}

A clean point contact provides a model system for the study of fundamental aspects of the Josephson CФR. As we shall see below, the supercurrent per single channel is a building block for more complex structures. A clean point contact is a constriction with a size in both directions (transverse and along the current) smaller than the electronic mean free path. The contact may contain a single scattering center, e.g., a tunnel barrier of finite transparency, characterized by the transmission coefficient $D$, where $0 \leqslant D \leqslant 1$. A highly idealized model for a clean constriction is the aperture in a thin impenetrable screen.

Kulik and Omelyanchuk (1977) advanced a theory, hereafter referred to as the KO-2 model, to describe the Josephson effect in the fully ballistic case, $D=1$. The supercurrent was calculated in the framework of the Eilenberger equations [Eqs. (A6)-(A8) in Appendix, Sec. 2]. For $D=1$, the boundary conditions, Eqs. (A9) and (A10), provide continuous matching of the Green's functions along the trajectories crossing the constriction. The Eilenberger equations can then be straightforwardly solved (see also discussion by Likharev, 1979), with the supercurrent given by the simple expression

$$
I_{S}(\varphi)=\frac{\pi \Delta}{e R_{N}} \sin (\varphi / 2) \tanh \frac{\Delta \cos (\varphi / 2)}{2 T} .
$$

The only characteristic of the weak-link material in Eq. (13) is the Sharvin resistance $R_{N}^{-1}=e^{2} k_{F}^{2} S /\left(4 \pi^{2} \hbar\right)$, where $k_{F}$ is the Fermi wave vector and $S$ is the constriction area.

The conductance of a ballistic point contact is quantized in units of $2 e^{2} / h$ (see the review by Beenakker and van Houten, 1991a). The generalization of Eq. (13) to the quantum regime was provided by Beenakker and van Houten (1991b) and by Furusaki and Tsukada $(1990,1991)$. It was shown theoretically that the critical current of a smooth and impurity-free superconducting constriction increases stepwise as a function of its width, with the step height at zero temperature being equal to $e \Delta / \hbar$. This result is a natural extension of Eq. (13), where the quantum resistance $R_{N}^{-1}=e^{2} / \pi \hbar$. If $N$ channels are open, the general expression for the supercurrent is

$$
I_{S}(\varphi)=N \frac{e \Delta}{\hbar} \sin (\varphi / 2) \tanh \frac{\Delta \cos (\varphi / 2)}{2 T} .
$$

Supercurrent quantization with a variation of the junction width was observed by Takayanagi et al. (1995b) in ballistic S/2DEG/S structures.

\section{Point contact, the general case}

Haberkorn et al. (1978) generalized the KulikOmelyanchuk result to the case of arbitrary transparency of a tunnel barrier inserted in the constriction by directly solving the Gor'kov equations (A1). The result is

$$
\begin{aligned}
I_{S}(\varphi)= & \frac{\pi \Delta}{2 e R_{N}} \frac{\sin \varphi}{\sqrt{1-\bar{D} \sin ^{2} \frac{\varphi}{2}}} \\
& \times \tanh \left[\frac{\Delta}{2 T} \sqrt{1-\bar{D} \sin ^{2} \frac{\varphi}{2}}\right],
\end{aligned}
$$

where $\bar{D}$ is the angle-averaged transmission probability and the contact resistance $R_{N}$ is

$$
R_{N}^{-1}=\frac{e^{2} k_{F}^{2} S}{4 \pi^{2} \hbar} \bar{D}
$$

Equation (15) interpolates nicely between a clean ballistic constriction and a tunnel junction. This expression reduces to the Aslamazov-Larkin result near $T_{c}$ and to the Ambegaokar-Baratoff result for a tunnel junction for $\bar{D} \ll 1$, which was later derived by several authors using different approaches (see Zaitsev, 1984; Arnold, 1985; Furusaki and Tsukada, 1990; Beenakker and van Houten, 1991b; Bagwell, 1992).

The modifications of the C $\Phi \mathrm{R}$ with varying average transparency and temperature given by Eq. (15) are shown in Figs. 4 and 5, respectively. The strongest devia- 


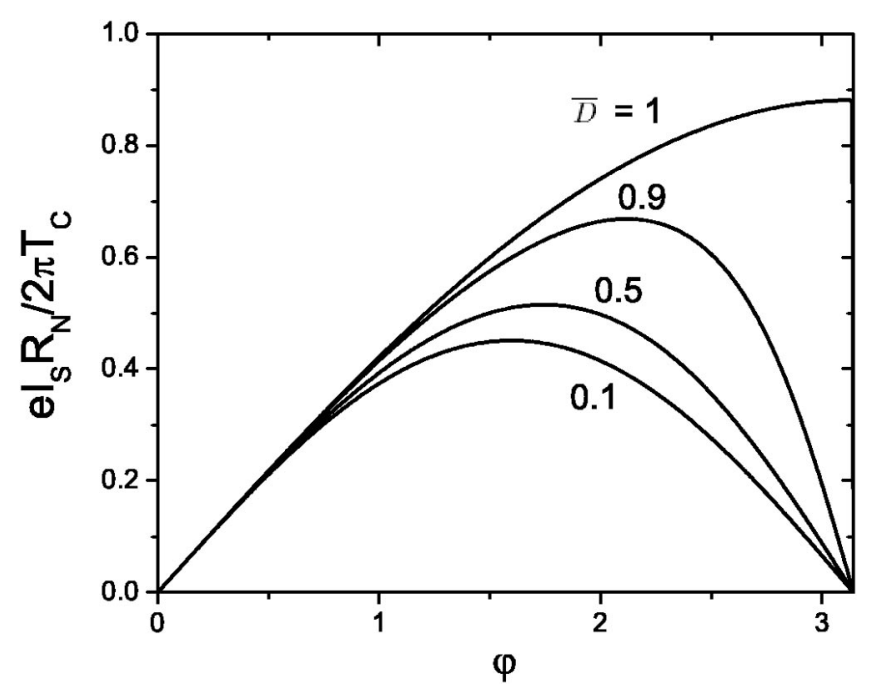

FIG. 4. Current-phase relations for a clean point contact at zero temperature and various values of the angle-averaged transmission probability $\bar{D}$.

tions from the sinusoidal form occur for ballistic contact with $\bar{D}=1$ at low $T$, when $I_{S}(\varphi) \propto \sin (\varphi / 2)$. There is a crossover to harmonic dependence with increasing temperature or decreasing $\bar{D}$. According to the above discussion, the CФR becomes sinusoidal for $\bar{D} \ll 1$ over the whole temperature range or near $T_{c}$ for arbitrary $\bar{D}$.

A multichannel generalization of Eqs. (15) and (14) was performed by Beenakker (1991; see also Böttcher and Kopp, 1997):

$$
I_{S}(\varphi)=\frac{e \Delta^{2}}{2 \hbar} \sin \varphi \sum_{n=1}^{N} \frac{D_{n}}{E_{n}} \tanh \left[\frac{E_{n}}{2 T}\right],
$$

where $D_{n}$ are the eigenvalues of an arbitrary transmission matrix that describes a disordered junction, $n$ is the number of channels, and $E_{n}=\Delta \sqrt{1-D_{n} \sin ^{2}(\varphi / 2)}$ is the energy of the Andreev bound state. Equation (17) is

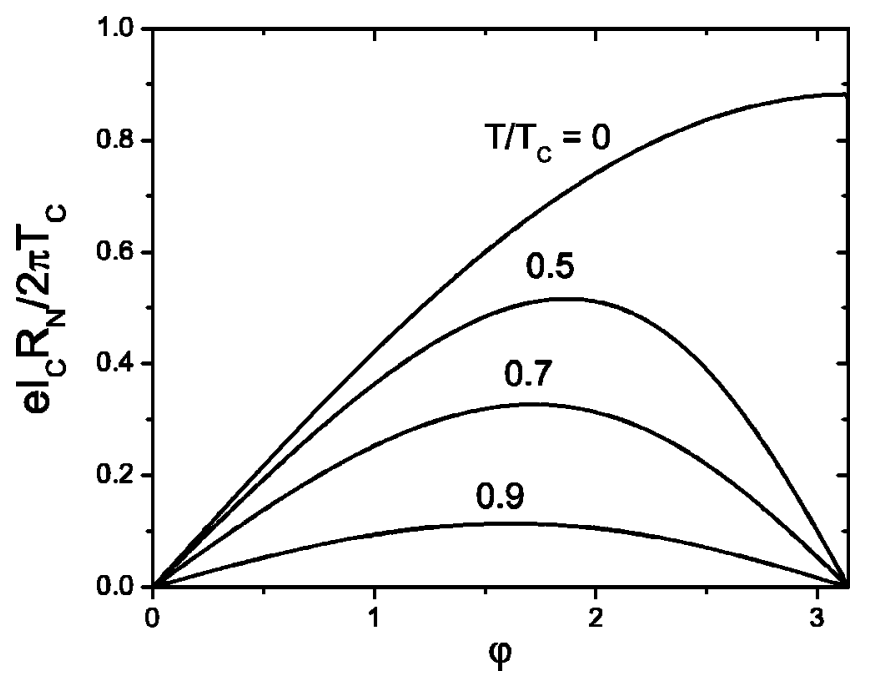

FIG. 5. Current-phase relations for a clean point contact for $D=1$ and various temperatures. valid for arbitrary degrees of disorder. If the number of channels $N$ is large, one can go from summation over $n$ to integration over transmissions $D$ according to $\sum_{n=1}^{N} \cdots=\int_{0}^{1} \rho(D) d D \cdots$, where $\rho(D)$ is the transmission eigenvalue density. In a diffusive point contact, when $l \ll \xi_{0}$, the eigenvalue density function is universal (Dorokhov, 1984; Nazarov, 1994a)

$$
\rho(D)=\left(\pi \hbar / 2 e^{2} R_{N}\right) D^{-1 / 2}(1-D)^{-1 / 2} .
$$

Integrating Eq. (15) with this function provides exactly the Kulik-Omelyanchuk expression for the supercurrent in the dirty limit, Eq. (11). Below, we shall discuss the applicability of this result to other types of short weak links. For a derivation based on the Eilenberger equations and further applications of nonstationary problems see Bardas and Averin (1997) and Zaitsev and Averin (1998).

The most general formula for a dc supercurrent through a classical point contact (large number of channels $N$ ) was derived by Zaitsev (1984). The theory of Zaitsev takes the asymmetry of the electrodes into account as well as possible non-BCS structure of the densities of states in the superconducting electrodes [e.g., due to the proximity effect or a magnetic field; see also Aminov et al. (1996) and Zaitsev and Averin (1998)]. As a model for the constriction, an aperture of small radius was considered in a thin impenetrable screen dividing two different superconductors. The angle-dependent transmission coefficient $D(\alpha)$ was introduced within the aperture, where $\alpha$ is the angle between the electronic trajectory and normal to the junction plane. Solving the Eilenberger equations [Eqs. (A6)-(A8) in the Appendix, Sec. 2] with the proper boundary conditions [Eqs. (A9) and (A10) in the Appendix, Sec. 2], Zaitsev (1984) derived the following general expression for the supercurrent:

$$
I_{S}(\varphi)=\frac{8 \pi T}{e R_{N}} \sum_{\omega>0}\left\langle\frac{f_{1} f_{2} \sin \varphi}{2+D\left(g_{1} g_{2}+f_{1} f_{2} \cos \varphi-1\right)}\right\rangle,
$$

where the angular brackets $\langle\cdots\rangle=\int_{0}^{1}(\cdots) x d x$ denote averaging over angle $\theta$ between electronic trajectories and the interface normal, $x=\cos \theta$. For a symmetric junction with bulk values for the Green's functions in both electrodes, $g_{1,2}=\omega / \sqrt{\omega^{2}+\Delta^{2}}$ and $f_{1,2}=\Delta / \sqrt{\omega^{2}+\Delta^{2}}$, the summation in Eq. (19) can be performed analytically and it reduces to the result of Haberkorn et al. (1978).

Equation (19) can be rewritten in the real energy representation of Eq. (2) with the equilibrium distribution function $f_{L}=\tanh (E / 2 T)$ and spectral supercurrent $\operatorname{Im} I_{S}(E)$ given by the analytical continuation of the expression in the angular brackets in Eq. (19) from Matsubara frequencies $i \omega=E+i 0$. For fixed $D$, the resulting expression has poles at energies

$$
E(\varphi)= \pm \Delta \sqrt{1-D \sin ^{2}(\varphi / 2)},
$$

corresponding to the Andreev bound state [see also Beenakker (1991) or Bagwell (1992), who derived this result from the Bogoliubov-de Gennes equations]. When we wish to distinguish the positive and negative 


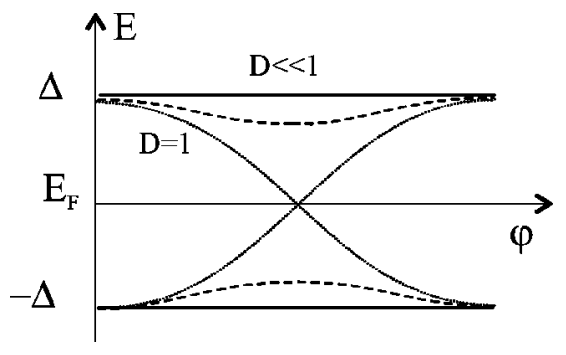

FIG. 6. Energy-phase relation for Andreev bound states in a short weak link. The energies of two bound states given by Eq. (20) are shown: solid lines, the tunneling limit (low transparency); dotted line, the ballistic regime $(D=1)$; dashed lines, the intermediate case.

poles, we use a subscript, $E_{ \pm}= \pm \Delta \sqrt{1-D \sin ^{2}(\varphi / 2)}$. The two signs describe two possible processes (one of them is shown in Fig. 1), which differ in the direction of propagation of the electrons and holes and correspond to currents flowing in opposite directions.

Coupling to an external magnetic field or supercurrent leads to a Doppler shift of the bound-state energies given by $\mathbf{v}_{F} \cdot \mathbf{p}_{S}$, where $\mathbf{v}_{F}$ is the Fermi velocity and $\mathbf{p}_{S}$ is the condensate momentum (see Fogelström et al., 1997, for details). In the problems considered in this review within the quasiclassical Green's-function technique, the Doppler shift is automatically taken into account and leads to depairing by the current.

Each bound state carries a current

$$
\begin{aligned}
I(\varphi) & =2 e f_{0}[E(\varphi)] \frac{d}{d \varphi} E(\varphi) \\
& =e f_{0}[E(\varphi)] D \frac{\Delta^{2}}{2 E(\varphi)} \sin (\varphi),
\end{aligned}
$$

where $f_{0}[E(\varphi)]$ is the Fermi occupation factor of the bound state. Therefore the factor $1-2 f=\tanh (E / 2 T)$ in Eq. (2) has a simple physical interpretation (Bagwell, 1992): it arises due to the difference in populations of two Andreev bound states, $\tanh (E / 2 T)=f_{0}\left(E_{+}\right)$ $-f_{0}\left(E_{-}\right)$, where $E=E_{+}=-E_{-}$. This can be viewed as a thermal average of the contributions of the two bound states (i.e., left- and right-moving electrons) to the supercurrent.

Figure 6 shows the Andreev bound state as a function of the phase difference $\varphi$ across a single-channel junction characterized by a single transmission coefficient $D$. In the ballistic regime $(D=1)$, two levels, corresponding to left-moving $\left(E_{+}\right)$and right-moving $\left(E_{-}\right)$electrons, cross at $\phi=\pi$, while the gap $2 \Delta \sqrt{1-D}$ opens for $D<1$.

As mentioned above, Eq. (19) is quite general and describes not only an ideal $\mathrm{ScS}$ junction, but also a junction with spatially inhomogeneous electrodes. Aminov et al. (1996) considered the practically interesting case of a SNcNS point contact, two NS bilayers coupled by a clean constriction with arbitrary transparency $D$. If the NS bilayers are in the dirty limit, the Green's functions $f_{1,2}$ and $g_{1,2}$ in Eq. (19) suitable for this problem are found from the Usadel equations [Eqs. (A11)-(A14) in
Appendix, Sec. 2]. For a symmetric SNcNS contact with thin normal layers, $d_{N} \ll \xi_{2}$, the supercurrent is given by the rather simple expression (Aminov et al., 1996)

$$
I_{S}(\varphi)=\frac{2 \pi T}{e R_{N}} \sum_{\omega>0} \frac{\Phi^{2}(\omega) \sin \varphi}{\omega^{2}+\left[1-D \sin ^{2}(\varphi / 2)\right] \Phi^{2}(\omega)} .
$$

Here the function $\Phi(\omega)=\Delta /\left[1+\left(\Delta^{2}\right.\right.$ $\left.\left.+\omega^{2}\right)^{1 / 2} \gamma_{B M} / \pi T_{c}\right]$, where $\gamma_{B M}=\gamma_{B} d / \xi_{2}$ with $\gamma_{B}$ given by Eq. (A19). Such a constriction model for SNcNS contacts was applied to interpret experimental data for some high- $T_{c}$ superconducting junctions and $\mathrm{S} / 2 \mathrm{DEG} / \mathrm{S}$ Josephson junctions by Aminov et al. (1996), Chrestin et al. (1997), Golubov et al. (1997), Golubov, Devyatov, et al. (1998), Schäpers (2001), and Grajcar, Ebel, et al. (2002).

At nonzero bias voltage, the mechanism of conduction in point contacts is the process of multiple Andreev reflection (Zaitsev, 1980; Klapwijk et al., 1982). A discussion of multiple Andreev reflection theory in short weak links is outside the scope of the present review. ${ }^{6}$

\section{JOSEPHSON JUNCTIONS WITH TUNNEL-TYPE CONDUCTIVITY}

The Josephson effect in a tunnel junction deals with the coherent transmission of Cooper pairs through a barrier which separates superconducting electrodes. The simplest case of an ideal tunnel junction, with the barrier modeled by a delta functional potential, was originally considered by Josephson (1962). Further developments in the theory include more complex situations, like extended barriers, resonant tunneling through localized states, and depairing by current.

\section{A. Ideal tunnel junctions}

Ambegaokar and Baratoff (1963) used the Green'sfunction method to generalize the calculation of Josephson (1962) to finite temperatures. They also explicitly pointed out that the Josephson effect occurs because the Cooper-pair amplitudes [the Gor'kov $F$ functions; see Eq. (A1)], which are nonlocally dependent on the superconducting pair potential $\Delta$, may overlap in the oxide layer even though $\Delta=0$ there.

The CФR in an SIS tunnel junction is sinusoidal and is given by the following expression (Ambegaokar and Baratoff, 1963):

\footnotetext{
${ }^{6}$ This theory was advanced by Arnold (1985), Gunsenheimer and Zaikin (1994), Averin and Bardas (1995), Bratus' et al. (1995), and Martin-Rodero et al. (1996). Multiple Andreev reflection was observed in various types of nanoscale contacts by Kleinsasser et al. (1994), van der Post et al. (1994), Vleeming et al. (1994), Takayanagi et al. (1995a, 1995b), Mur et al. (1996), Scheer et al. (1997, 2001), Ludoph et al. (2000), Naveh et al. (2000), Buitelaar et al. (2002, 2003), and Agrait et al. (2003).
} 


$$
\frac{e I_{S}(\varphi) R_{N}}{2 \pi T_{c}}=\frac{T}{T_{c}} \sum_{\omega>0} \frac{\Delta_{1} \Delta_{2}}{\sqrt{\left(\omega^{2}+\Delta_{1}^{2}\right)\left(\omega^{2}+\Delta_{2}^{2}\right)}} \sin \varphi .
$$

This result can be also obtained directly from Eq. (19) in the limit $D \ll 1$.

The Ambegaokar-Baratoff formula (23) is a particular case of the general theory formulated by Larkin and Ovchinnikov (1966) and Werthamer (1966) for an SIS tunnel junction at arbitrary constant bias voltage $V$,

$$
\begin{aligned}
I_{S}= & \frac{\sin \varphi}{2 e R_{N}} \int_{-\infty}^{\infty}\left\{\operatorname{Re} F_{1}(E) \operatorname{Im} F_{2}(E+e V)\right. \\
& \left.+\operatorname{Im} F_{1}(E) \operatorname{Re} F_{2}(E+e V)\right\} \tanh (E) d E,
\end{aligned}
$$

where the time-dependent phase difference $\varphi=2 \mathrm{eVt}$ $+\varphi_{0}$ and $F_{1,2}$ are the quasiclassical Green's functions in superconductors, $F_{1,2}=\Delta_{1} / \sqrt{\Delta_{1,2}^{2}-E^{2}}$ [compare with Eq. (A15) for $\omega=-i E]$. In the limit $V \rightarrow 0$, Eq. (24) is reduced to Eq. (23).

The physical mechanism of a supercurrent transfer across the tunnel barrier can be understood in terms of Andreev bound states. Comparing Eq. (24) with Eq. (2), one can see that in an asymmetric tunnel junction with $\left|\Delta_{1}\right| \leqslant\left|\Delta_{2}\right|$ all states with energies in the interval $\Delta_{1}$ $\leqslant|E| \leqslant \Delta_{2}$ contribute to the spectral current. That means that the Andreev bound states are distributed over the whole energy range between $\left|\Delta_{1}\right|$ and $\left|\Delta_{2}\right|$. In a symmetric tunnel junction $\left(\Delta_{1}=\Delta_{2}=\Delta\right)$ the bound-state energies are $E(\varphi)= \pm \Delta$ (see Fig. 6) and, in accordance with Eq. (23), the supercurrent

$$
I_{S}(\varphi)=\frac{e \Delta}{2} D \tanh \frac{\Delta}{2 T} \sin (\varphi)
$$

is proportional to the normal electron transparency of the junction.

In tunnel junctions with extended barriers, supercurrent transport is more complex due to the existence of a resonant coupling between superconducting surface states, situated at the two SI interfaces of the SIS junction (Furusaki and Tsukada, 1991; Wendin and Shumeiko, 1996a, 1996b) and the appearance of localized states inside the barrier. ${ }^{7}$ The physics of resonant tunneling will be discussed below.

\section{B. Tunnel junctions with extended barriers}

In junctions with a thick tunnel barrier having a small transparency $D \ll 1$, the structure of the bound-state spectrum is determined by the coupling of the supercon-

\footnotetext{
${ }^{7}$ See Knauer, 1977; Itskovich and Shekhter, 1981; Aslamazov and Fistul', 1982; Larkin and Matveev, 1987; Glazman and Matveev, 1988, 1989; Tartakovsky and Fistul', 1988; Beenakker and van Houten, 1991a, 1991b, 1991c; Devyatov and Kupriyanov, 1993, 1994a, 1994b, 1997a, 1997b, 1998; Devyatov, Goncharov, et al., 1995, 2001a, 2001b; Aleiner, 1996; Devyatov, Kupriyanov, and Wendin, 1997; Naveh et al., 2000; Halbritter, 2002.
}

ducting surface states situated at the two SI interfaces of the SIS junction (Wendin and Shumeiko, 1996a, 1996b):

$$
\begin{aligned}
& E^{2}(\varphi)=\Delta^{2}\left[1-\Omega^{2}\right], \quad \Omega=\left(\frac{|\beta|}{2} \pm \sqrt{D}\left|\sin \frac{\varphi}{2}\right|\right), \\
& \varphi \gg d \delta k, \\
& \delta k=\{\sqrt{2 m[W-(\mu+E)]}-\sqrt{2 m[W-(\mu-E)]}, \\
& \beta \approx-2 k_{s} \delta k /\left(k_{s}^{2}+k_{b}^{2}\right), \quad|\beta| \ll 1 .
\end{aligned}
$$

Here $k_{s}=\sqrt{2 m \mu}, \quad k_{b}=\sqrt{2 m W}, m$ is the effective mass, $W$ is the height of the barrier, $\mu$ is the Fermi energy, and $E$ is the quasiparticle energy.

Each bound state carries a large current,

$$
\begin{aligned}
I_{S}\left[E_{ \pm}(\varphi)\right]= & \mp n_{F}[E(\varphi)] \frac{e \Delta D}{2} \sin (\varphi) \\
& \times\left(1 \pm \frac{|\beta|}{2 \sqrt{D}} \frac{1}{\sqrt{\sin ^{2}(\varphi / 2)+(\delta k d / 2)^{2}}}\right) .
\end{aligned}
$$

The magnitude of $I\left[E_{ \pm}(\varphi)\right]$ is proportional to the amplitude rather than the probability of normal electron tunneling. Nevertheless, the currents are distributed among the bound states in such a way that they almost cancel each other in equilibrium, giving rise to a comparatively small residual current, including the contribution from the continuum. This current coincides with the Ambegaokar-Baratoff expression (26).

The large current of a single bound state as well as a nonsinusoidal CФR can be revealed under nonequilibrium conditions, when the bound level population is unbalanced by means of microwave pumping or tunnel injection.

\section{Tunneling via localized states}

The problem of supercurrent transfer across a Josephson tunnel junction containing resonant impurity levels in the tunnel barrier, the so-called localized electronic states, was first considered by Aslamazov and Fistul' (1982). They showed that resonant propagation of coherent electrons along trajectories made up of periodically arranged impurity atoms results in a slower decrease of the critical current with increasing barrier thickness than occurs with direct tunneling. The CФR remains sinusoidal; in this model the localized states form long metallic channels so that the junction is similar to a long SNS junction that has a sinusoidal CФR.

Deviations from the $\sin (\varphi)$ law may occur if the charge transport is via a single localized state (Larkin and Matveev, 1987; Glazman and Matveev, 1989; Beenakker and van Houten, 1991a, 1991b, 1991c; Devyatov and Kupriyanov, 1997b, 1998; Devyatov et al., 1997; Naveh et al., 2000).

Devyatov and Kupriyanov (1997b) have shown that the properties of the structure depend on the relation of the barrier thickness $d$ and the decay lengths $\xi_{i}$ and $\xi_{b}$, 


$$
\xi_{i}=\frac{\sqrt{W-\mu}}{2 m E}, \quad \xi_{b}=\frac{m}{\sqrt{W-\mu}} \ln \frac{W-\mu}{E} .
$$

If the thickness of the tunnel barrier $d \ll \xi_{i}, \xi_{b}$, then the Andreev bound-state spectrum is determined by the equation (Devyatov and Kupriyanov, 1997b)

$$
\begin{aligned}
& \sqrt{\Delta^{2}-E^{2}}\left(E^{2}-E_{R}^{2}-\frac{\Gamma^{2}}{4}\right)+\frac{\Delta^{2} \Gamma_{1} \Gamma_{2}}{\sqrt{\Delta^{2}-E^{2}}} \sin ^{2}\left(\frac{\varphi}{2}\right)+\Gamma E^{2} \\
& \quad=0 \\
& \Gamma=\Gamma_{1}+\Gamma_{2}, \quad \Gamma_{1}=\Gamma_{0} \exp \left(-\frac{x_{0}}{\alpha}\right), \\
& \Gamma_{2}=\Gamma_{0} \exp \left(\frac{x_{0}}{\alpha}\right),
\end{aligned}
$$

where $\alpha=[2 m(W-\mu)]^{-1 / 2}$ is the inverse radius of the localized state, $\Gamma_{0}=2(W-\mu) \sqrt{D}$ is the width of the state's resonant level, $E_{R}$ is the renormalized energy, and $x_{0}$ is the position of the state inside the barrier. Equation (30) was obtained previously by Beenakker and van Houten (1991a, 1991b, 1991c) for the problem of tunneling via a quantum dot that connects two superconductors.

In the limit of a broad resonance $\Gamma_{0} \gg \Delta$, i.e., in the range of barrier thickness

$$
\alpha \ll d \ll \alpha \ln \left[8 \frac{W-\mu}{\Delta} \sqrt{\frac{W-\mu}{\mu}}\right],
$$

the dispersion relation (30) yields an expression for the energy of the Andreev bound state similar to Eq. (20) in constrictions,

$$
E(\varphi)= \pm \Delta \sqrt{1-D\left(E_{R}\right) \sin ^{2}\left(\frac{\varphi}{2}\right)}
$$

with the coefficient $D$ replaced by $D\left(E_{R}\right)$,

$$
D\left(E_{R}\right)=\frac{\Gamma_{0}^{2}}{\left(E-E_{R}\right)^{2}+\Gamma_{0}^{2} \cosh ^{2}\left(x_{0} / \alpha\right)},
$$

describing the Breit-Wigner resonance for a localized state with an energy $E_{R}$.

Equations (21) and (32) immediately provide the Josephson current through the structure (Glazman and Matveev, 1989),

$$
I_{S}(\varphi)=\frac{e \Delta^{2}}{2} D\left(E_{R}\right) \frac{\sin (\varphi)}{|E(\varphi)|} \tanh \frac{|E(\varphi)|}{2 T},
$$

which is formally the same as the CФR for a singlemode ScS constriction (Haberkorn et al., 1978).

In the opposite case of a narrow resonance, $\Gamma_{0} \ll \Delta$, both $E(\varphi)$ and $I_{S}(\varphi)$ have the same dependence on $\varphi$ as in Eqs. (32) and (34), respectively, but with a strongly suppressed prefactor,

$$
E(\varphi)= \pm \frac{\Delta \Gamma_{0} \sqrt{1-D\left(E_{R}\right) \sin ^{2}(\varphi / 2)}}{\sqrt{D\left(E_{R}\right)\left\{E_{R}^{2}+\left[\Delta+\Gamma_{0} \cosh \left(x_{0} / \alpha\right)\right]^{2}\right\}}},
$$

$$
I_{S}(\varphi)=\frac{e \Delta^{2} \Gamma_{0} \sqrt{D\left(E_{R}\right)} \sin (\varphi) \tanh [|E(\varphi)| / 2 T]}{2|E(\varphi)| \sqrt{\left\{E_{R}^{2}+\left[\Delta+\Gamma_{0} \cosh \left(x_{0} / \alpha\right)\right]^{2}\right\}}} .
$$

Equations (34) and (36) imply that in both limiting cases $I_{S}(\varphi)$ differs from sinusoidal for localized-state energies $E_{R}<\Gamma_{0}$. In particular, for $E_{R}=0$ and $x_{0}=0$ the structure is fully transparent and $I_{S}(\varphi) \propto \sin (\varphi / 2)$ at $T=0$ in the two cases of broad or narrow resonance.

To calculate the supercurrent one has to average Eq. (34) over the coordinates and energies of the localized state,

$$
\left\langle I_{S}(\varphi)\right\rangle=\int I_{S}(\varphi) \rho\left(E_{R}, x_{0}\right) d x_{0} d E_{R},
$$

where $\rho\left(E_{R}, x_{0}\right)$ is the distribution function.

Naveh et al. (2000) have shown that if $\Gamma_{0}$ in Eq. (33) is so large that the first term in the denominator is unimportant, then $D\left(E_{R}\right)$ depends only on the position of the localized state inside the barrier, $x_{0}$. To calculate a current it is enough to average Eq. (34) only over $x_{0}$. Then $D\left(E_{R}, x_{0}\right) \approx \cosh \left(x_{0} / \alpha\right)^{-2}$ and, for a system with a uniform spatial distribution of localized states, $\rho\left(E_{R}, x_{0}\right)=\rho\left(x_{0}\right)=$ const. Averaging over the positions of the localized states,

$$
d x_{0}=\left(\frac{d D}{d x_{0}}\right)^{-1} d D \equiv \rho(D) d D,
$$

is equivalent to averaging the supercurrent in Eq. (34) with Dorokhov's distribution function $\rho(D)$ given by Eq. (18). This provides exactly the result of the KO-1 theory, Eq. (11).

If, on the other hand, the localized states are distributed uniformly in energy and are located in the middle of the barrier, so that $x_{0} \approx 0$ and $D\left(E_{R}, x_{0}\right) \approx\{1+[(E$ $\left.\left.\left.-E_{R}\right)^{2} / \Gamma_{0}^{2}\right]\right\}^{-1}$, then

$$
d E_{R}=\left(\frac{d D}{d E_{R}}\right)^{-1} d D \equiv \rho(D) d D
$$

with the distribution function

$$
\rho(D)=\left(\hbar / e^{2} R_{N}\right) D^{-3 / 2}(1-D)^{-1 / 2},
$$

as derived by Schep and Bayer (1997a, 1997b) for disordered interfaces. This averaging provides the temperature dependence of the supercurrent, is a good fit with the experimental data of Naveh et al. (2000) for high- $J_{C}$ SIS junctions. The same distribution is also valid for double-barrier junctions (Beenakker, 1997), for which the averaging gives exactly the result of Kupriyanov and Lukichev (1988a, 1998b; see Sec. VI below) for SINIS junctions in the coherent regime, Eq. (118). Therefore the form of the supercurrents in high- $J_{C}$ SIS and SINIS junctions in the coherent regime are identical, though the corresponding physical mechanisms are different.

If $\rho\left(E_{R}, x_{0}\right)$ is a function of both arguments, then averaging the supercurrent of Eq. (34) over the coordinates and energies of the localized-state results in $I_{S}(\varphi) \propto \sin (\varphi)$ over almost the entire temperature range. 


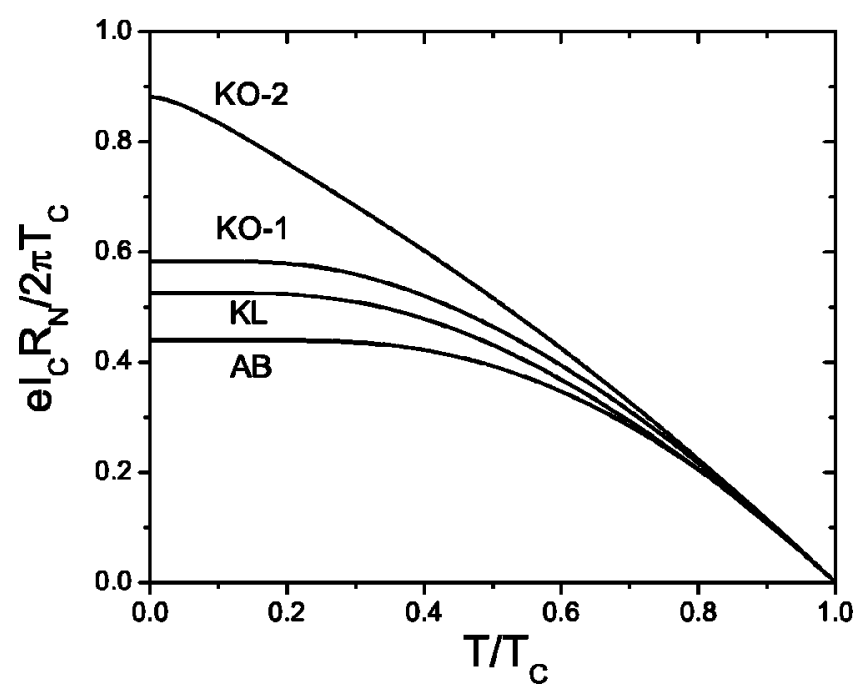

FIG. 7. Temperature dependencies of the critical current for various types of short weak links.

An averaging procedure carried out in the threedimensional case using the Green's function formalism yields the same result (Glazman and Matveev, 1989; Devyatov and Kupriyanov, 1994a).

A comparison of the temperature dependencies of the critical current for different types of short weak links and tunnel junctions is shown in Fig. 7. At $T=0$, the $I_{C} R_{N}$ product for a ballistic $\mathrm{ScS}$ point contact (KO-2) is twice as large as for a tunnel junction (Ambegaokar and Baratoff, 1963). The $I_{C} R_{N}$ product for a diffusive point contact (KO-1) and a double-barrier junction in the coherent (Kupriyanov and Lukichev) regime have intermediate values between KO-1 and the Ambegaokar and Baratoff equation (23).

It can be shown that a further increase of the barrier thickness $\left(\xi_{b} \ll d \ll \xi_{i},\right)$ leads to the appearance of two additional Andreev levels located in the vicinity of $E$ $=\Delta$ (Devyatov and Kupriyanov, 1997b). These two new solutions are direct analogs of the split Andreev levels examined by Wendin and Shumeiko (1996a, 1996b) for the case of a long SIS junction and discussed earlier. The expressions for $E(\varphi)$ and $I_{S}(\varphi)$ obtained in this limit have the same form as Eqs. (27) and (28) with the factors $D$ and $\sin (\varphi / 2)$ replaced by the resonance transparency in Eq. (33) and $\cos (\varphi / 2)$, respectively. Similarly, the large currents carried by the individual levels flow in opposite directions and effectively cancel each other. The resulting current appears to be exponentially small as compared to the contribution from the lower energy levels in Eq. (36) and does not affect resonant transport.

A further increase in the junction thickness does not affect the expression for the Andreev level [Eq. (35)] near the chemical potential $\left(E_{R} \approx \Gamma_{0}\right)$. A numerical analysis (Devyatov and Kupriyanov, 1997b) has shown that, as before, two split roots exist near $E=\Delta$, but they become flatter and, as before, their presence does not contribute to the full equilibrium resonant Josephson current determined by Eq. (36).

The CФR associated with tunneling via individual localized states has the same form as for a geometrical constriction. Therefore a localized state can be considered to be a constriction in energy space. The contribution of an individual localized state as a function of $\Gamma_{0}$ saturates at large $\Gamma_{0} \gg \Delta$, while at small $\Gamma_{0}$ it scales as $\Gamma_{0} / \Delta$.

The experimental observation of deviations of $I_{S}(\varphi)$ from $\sin \varphi$ in tunnel structures is difficult since, in real SIS junctions, the resonant contributions to the full supercurrent are effectively averaged over the positions and energies of the localized state inside the barrier. Together with the contribution from the direct tunneling channel, this results in a $\sin (\varphi)$ dependence with a critical current larger than prediction of the AmbegaokarBaratoff theory [Eq. (23)].

Nevertheless, nonsinusoidal $I_{S}(\varphi)$ can be observed in high- $J_{C}$ tunnel junctions (Naveh et al., 2000) or as a result of depairing by the supercurrent in the electrodes, which may occur for an increased barrier transparency in in-line Josephson junctions with a very narrow electrode width.

\section{SIS structures with high transparency: Depairing by current}

Depairing by current generally occurs when the barrier transparency is high. Due to self-consistency (coupling across the barrier), the pair potential near the interface is suppressed more strongly than the bulk pair potential. As a result, with increasing current, depairing at the interface is achieved faster than the depairing in the bulk. Such a crossover from the Josephson effect to bulk superconducting flow was first analyzed quantitatively by Kupriyanov (1992) for SIS junctions with high barrier transparency. Similar results were obtained by Sols and Ferrer (1994) and Freericks et al. (2002) for a wider class of junctions and using different models.

The supercurrent across an SIS structure with arbitrary transparency $D$ can be obtained from the Usadel equation (A23), with boundary conditions Eqs. (A25) and (A26) at the barrier going to a spatially homogeneous current-carrying state far from the SIS interface (Kupriyanov, 1992). The problem is simplified by the fact that, in symmetric junctions, the boundary conditions (A25) and (A26) do not contain Matsubara frequencies and, as in the depairing problem (Kupriyanov and Lukichev, 1980), the phases of all Usadel functions can be chosen equal to the phase of $\Delta$ in the electrodes.

With this simplification, it can be shown that at $T$ $\lesssim T_{c}$ the CФR can be found from the first integral of the Ginzburg-Landau equation,

$$
\begin{aligned}
& \left(\frac{2 \alpha \sin ^{2}(\varphi / 2)}{\Gamma_{B}}\right)^{2}+(a-1) \\
& \quad \times\left[1-\frac{I^{2} \Delta_{0}^{4}}{a I_{0}^{2} \Delta^{4}(\infty)}-\frac{\Delta^{2}(\infty)(1+a)}{2 \Delta_{0}^{2}}\right]=0,
\end{aligned}
$$

where $a$ is a parameter defined in Eq. (41). The supercurrent is given by

$$
I=I_{0} v_{S}\left(1-v_{S}^{2}\right), \quad I_{0}=\frac{\pi \Delta_{0}^{2}}{4 e \rho T_{c} \xi(T)},
$$




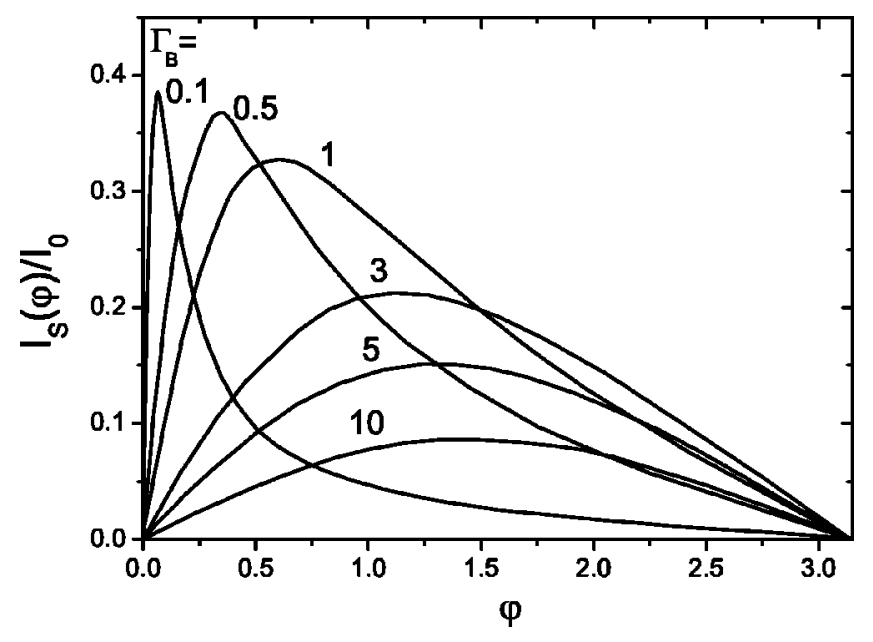

FIG. 8. The CФR for a superconductor-insulatorsuperconductor (SIS) junction for various values of the barrier transparency parameter $\Gamma_{B}$. Adapted from Kupriyanov, 1992.

where $\Delta(\infty)$ is the magnitude of the pair potential far from the interface,

$$
\Delta^{2}(\infty)=\Delta_{0}^{2}\left(1-v_{S}^{2}\right), \quad v_{S}=a \frac{\sin (\varphi)}{\Gamma_{B}}, a=\frac{\Delta(0)}{\Delta(\infty)} .
$$

Here $\Delta_{0}^{2}=\left[8 \pi^{2} / 7 \zeta(3)\right] T_{c}\left(T_{c}-T\right), \Gamma_{B}=\gamma_{B} \xi(T) / \xi$ is the suppression parameter, and $\Delta(0)$ is the magnitude of the pair potential at the interface.

In the limit of small transparency $\left(\Gamma_{B} \gg 1\right)$ in a firstorder approximation on $\Gamma_{B}^{-1}$, it follows from Eq. (39) that parameter $a=1$ and the result of the AmbegaokarBaratoff equation (26) for the supercurrent is reproduced. Corrections to this result are caused by the suppression of $\Delta(\infty)$ and $\Delta(0)$ by supercurrent. The last of these factors is more essential at $\Gamma_{B} \gg 1$ and leads to corrections to the critical current,

$$
I_{C}=I_{0} \Gamma_{B}^{-1}\left(1-\sqrt{2} \Gamma_{B}^{-1}\right)
$$

and small deformations of $I_{S}(\varphi)$ having a maximum at $\varphi_{\max }=\pi / 2-\sqrt{2} \Gamma_{B}^{-1}<\pi / 2$.

In the opposite limit $\left(\Gamma_{B} \ll 1\right)$ the $I_{S}(\varphi)$ is mainly controlled by depairing in the electrodes. The critical state should be achieved at $I \approx I_{0}$ and it follows from Eqs. (40) and (41) that $I \approx I_{0} \approx I_{0} \Gamma_{B}^{-1} \sin (\varphi)$. Therefore in this limit the position of the maximum of $I_{S}(\varphi)$ must be shifted to the value $\varphi_{\max } \approx \Gamma_{B}^{-1} \ll 1$. The calculations provide the result

$$
I_{S}(\varphi)=\left\{\begin{array}{l}
\beta\left(1-\beta^{2}\right), \quad 0 \leqslant \varphi \leqslant \varphi_{\max } \\
q\left(1-q^{2}\right), \quad \varphi_{\max } \leqslant \varphi \ll 1 \\
\Gamma_{B} / 4 \cot (\varphi / 2), \quad \varphi_{\max } \ll \varphi \leqslant \pi,
\end{array}\right.
$$

where $\beta=\varphi / \Gamma_{B}$ and $q^{-1}=\beta+\sqrt{1+\beta^{2}}$. The maximum $I_{S}(\varphi)$ is achieved at $\varphi_{\max }=\Gamma_{B} / \sqrt{3} \ll 1$ and the critical current equals the depairing current $I_{C}=(2 / 3 \sqrt{3}) I_{0}$.

The $I_{S}(\varphi)$ dependencies for arbitrary $\Gamma_{B}$ have been obtained numerically by Kupriyanov (1992) and are shown in Fig. 8 [see also Sols and Ferrer (1994) and Freericks et al. (2002)]. In full agreement with the above analysis, a decrease of $\Gamma_{B}$ leads to a transition from the $\sin (\varphi)$ law to the $I_{S}(\varphi)$ defined by Eq. (43). At $\Gamma_{B} \approx 10$ (corresponding to the specific barrier resistance $R_{B}$ $\approx 10 \rho \xi)$, the $\varphi_{\max }$ shifts more than $10 \%$ from $\pi / 2$.

For typical low-temperature superconducting materials, this value $R_{B} \approx 10 \rho \xi$ is $\sim 10^{-11} \Omega \mathrm{cm}^{2}$. Such values have been found experimentally for $\mathrm{Nb} / \mathrm{Cu}$ (Baxter et al., 1999; Park et al., 2000), Nb/Al (Dmitriev, 1999; Zehnder et al., 1999; Brammertz et al., 2001; den Hartog et al., 2001), and $\mathrm{Pd} / \mathrm{Cu}$ and $\mathrm{Pt} / \mathrm{Cu}$ interfaces (Kurt et al., 2002).

\section{SNS JUNCTIONS}

SNS Josephson junctions are structures composed of two superconducting electrodes connected by a normal metal, which may be a superconductor with a smaller critical temperature $T_{c}^{\prime}$. These structures are further classified as "clean" $\left(\ell \gg \xi_{0}, d\right)$ and "dirty" $\left(\ell \ll \xi_{0}, d\right)$ depending on the relationship between the electron mean free path $\ell$, the order parameter decay length in the interlayer material $\xi_{0}$, and the distance $d$ between electrodes.

Various types of SNS junction geometries are described by Likharev (1979) and include SNS sandwiches, variable-thickness bridges, and ramp junctions. The theory presented below deals with one-dimensional geometry, when depairing by supercurrent flow and selfconsistency of the pair potential across the structure should be taken into account. In structures with current concentration (variable-thickness bridges and ramp junctions), depairing does not play an essential role.

The shape of $I_{S}(\varphi)$ in SNS junctions depends on a variety of parameters: the ratio of the decay length in the interlayer material and distance between electrodes, the ratio of the critical temperatures $T_{c}$ and $T_{c}^{\prime}$, and the transport properties of the interfaces. We shall see that there are several practically important limits in which $I_{S}(\varphi) \propto \sin (\varphi)$. Deviations resulting in a shift of $\varphi_{\max }$ to the region $\varphi_{\max } \geqslant \pi / 2$ are controlled mainly by processes in the interlayer material, while $\varphi_{\max } \leqslant \pi / 2$ occurs due to processes in the electrodes.

To demonstrate this, let us start with an analysis of the simplest situation: electrodes with equal critical temperatures, $T_{c}^{\prime}=0, T \leq T_{c}$, both $\mathrm{S}$ and $\mathrm{N}$ in the dirty limit, and a junction width smaller than the Josephson penetration depth $\lambda_{J}$.

\section{A. The limit of high temperature, $T \approx T_{c}$}

An analysis of the shape of the CФR at temperatures close to $T_{c}$ was carried out by Ivanov et al. $(1978,1981)$, who showed that, in the practically interesting limit

$$
\gamma \ll \gamma_{B}+\min \left\{1, d / \xi_{2}\right\},
$$

where $\xi_{2}$ is the coherence length in the normal metal, the shape of $I_{S}(\varphi)$ may be found from the solution of two algebraic equations [see the definitions of $\gamma, \gamma_{B}$ in Eqs. (A21) and (A16)]. One of them follows from the first integral of the Ginzburg-Landau equations, 


$$
\begin{aligned}
\Gamma^{2} a^{2} & {\left[p \cos ^{2}(\varphi / 2)+q \sin ^{2}(\varphi / 2)\right]^{2}+(a-1) } \\
\times & {\left[1-\left(\frac{I_{S}^{2}(\varphi) \Delta_{0}^{4}}{a I_{0}^{2} \Delta^{4}(\infty)}\right)-\frac{\Delta^{2}(\infty)(1+a)}{2 \Delta_{0}^{2}}\right]=0 . }
\end{aligned}
$$

The other equation follows from the expression for the supercurrent,

$$
I_{S}(\varphi)=\frac{a}{2} I_{0} \Gamma(q-p) \frac{\Delta^{2}(\infty)}{\Delta_{0}^{2}} \sin (\varphi) .
$$

Here $a=\Delta(d / 2) / \Delta(\infty)$ and $\Delta(d / 2), \Delta(\infty)$ are the magnitudes of the pair potentials at the NS interface and in the bulk, respectively, $\Gamma=\gamma \xi(T) / \xi_{2}$ is the suppression parameter, and the functions $p\left(\gamma_{B}, d\right)$ and $q\left(\gamma_{B}, d\right)$ are defined by

$$
\begin{aligned}
& p \sum_{\omega>0} \omega^{-2}=\sum_{\omega} \frac{\omega^{-2} \beta}{\beta \gamma_{B}+\operatorname{coth}\left(\beta d / 2 \xi_{2}\right)}, \\
& q \sum_{\omega>0} \omega^{-2}=\sum_{\omega} \frac{\omega^{-2} \beta}{\beta \gamma_{B}+\tanh \left(\beta d / 2 \xi_{2}\right)},
\end{aligned}
$$

where $\beta=\sqrt{\pi T_{c} / \omega}$.

1. Weak depairing in the electrodes (rigid boundary conditions)

It follows from Eq. (45) that the critical current is smaller than $I_{0}$ and, in first approximation, $\Delta(d / 2)$ $=\Delta(\infty)=\Delta_{0}$ under the conditions $\Gamma q \ll 1$ and $\Gamma p \ll 1$, i.e., when

$$
\Gamma \ll \gamma_{B}+\min \left\{1, d / \xi_{2}\right\} .
$$

Hence, in the considered limit, the C $\Phi \mathrm{R}$ is sinusoidal (see Kupriyanov and Lukichev, 1988a, 1988b; Heslinga and Klapwijk, 1993), with $I_{C}$ given by

$$
I_{C}=I_{0} \frac{8 \Gamma}{\pi^{2}} \sum_{\omega>0} \frac{\beta^{-3} \sinh ^{-1}\left(\beta d / \xi_{2}\right)}{\left[1+\beta^{2} \gamma_{B}^{2}+2 \beta \gamma_{B} \operatorname{coth}\left(\beta d / \xi_{2}\right)\right]} .
$$

In the limit of small $\gamma_{B}$, Eq. (50) coincides with the result of Likharev (1976) for variable-thickness SNS bridges.

The normal resistance of the junction is the sum of the resistances of the weak-link material, $\widetilde{R}_{N}=\rho_{2} d / S$, and the boundary resistances,

$$
R_{N}=\frac{2 R_{B}+\rho_{2} d}{S}=\widetilde{R}_{N}\left(1+2 \Gamma_{B}\right), \quad \widetilde{\Gamma}_{B}=\gamma_{B} \frac{\xi_{2}}{d},
$$

where $S$ is the cross-section area of the interlayer, which is assumed to be equal to the area of the interfaces.

The $I_{C} R_{N}$ product decreases with increasing $\gamma_{B}$. In particular, for $d \ll \xi_{2}$ it follows from Eqs. (50) and (51) that

$$
\begin{aligned}
& I_{C} R_{N}=V_{0}\left\{1-\frac{4}{a \pi^{2}}\left[\psi\left(\frac{1+\vartheta}{2}\right)-\psi\left(\frac{1}{2}\right)\right]\right\}, \\
& V_{0}=\frac{\pi \Delta_{0}^{2}}{4 e T_{c}}, \quad \vartheta=\frac{1+\widetilde{\Gamma}_{B}}{\gamma_{B}^{2}}
\end{aligned}
$$

where $\psi(x)$ is the digamma function. It follows from Eq. (52) that decreasing transparency leads to a decrease in the slope of $I_{C} R_{N}(T)$ for $T \approx T_{c}$,

$R_{N} \frac{d I_{C}}{d T}= \begin{cases}635\left(1-\frac{4}{\vartheta \pi^{2}} \ln \frac{\vartheta}{2}\right)\left[\frac{\mu V}{K}\right], & \gamma_{B}^{2} \ll 1+\frac{\gamma_{B} \xi_{2}}{d} \\ 1082 \vartheta\left[\frac{\mu V}{K}\right], & \gamma_{B} \gg \frac{\xi_{2}}{d} .\end{cases}$

Equations (52) and (53) allow one to estimate the interface resistance from $I_{C}(T)$ data.

In the limit of large thickness, $d \gg \xi_{2}$, both the critical current and the $I_{C} R_{N}$ product are exponentially small,

$$
I_{C} R_{N}=V_{0} \frac{16}{\pi^{2}} \frac{1+2 \widetilde{\Gamma}_{B}}{\left(1+\gamma_{B}\right)^{2}} \frac{d}{\xi_{2}} \exp \left\{-\frac{d}{\xi_{2}}\right\}, \quad d \gg \xi_{2} .
$$

It follows from Eqs. (51), (53), and (54) that the $I_{C} R_{N}$ product is independent of weak-link transport parameters only if $\gamma_{B}$ is relatively small,

$$
\gamma_{B} \ll \max \left\{1, \xi_{2} / d\right\} .
$$

In the opposite parameter range, $I_{C}$ is proportional to $\gamma_{B}^{-2}$ and $R_{N} \propto \gamma_{B}$ for large $\gamma_{B}$, leading to $I_{C} R_{N} \propto R_{N}^{-1}$.

2. Depairing in superconducting electrodes by the proximity effect

It follows from Eqs. (47) and (48) that $\Gamma p$ and $\Gamma q$ are greater than 1 under the condition

$$
\Gamma \gg \gamma_{B}+\min \left\{1, d / \xi_{2}\right\} .
$$

In this regime one can neglect the magnitude of $\Delta(d / 2) \propto \Delta_{0} / \max (\Gamma p, \Gamma q)$ in comparison with $\Delta(\infty)$ in Eq. (46). Further, $\Delta(\infty) \simeq \Delta_{0}$ due to the smallness of the current through the superconductor $I \propto I_{0} /(\Gamma p, \Gamma q)$. With the simplifications made, we obtain directly from Eqs. (45) and (46) that

$$
I_{S}(\varphi)=I_{C} \frac{q p \sin (\varphi)}{p \cos ^{2}(\varphi / 2)+q \sin ^{2}(\varphi / 2)}, \quad \frac{I_{C}}{I_{0}}=\frac{q-p}{4 \Gamma q p} .
$$

The critical current $I_{C}$ is proportional to $\left(T-T_{c}\right)^{2}$, achieved at $\varphi=\arctan (q / p)$. Its asymptotic behavior at small and large values of $d$ is given by the expressions

$\frac{I_{C}}{I_{0}}=\frac{\pi^{2}}{8 \Gamma\left(1+\gamma_{B}\right)^{2}}\left[\sum_{\omega} \frac{1}{\beta^{3}\left(1+\beta \gamma_{B}\right)}\right]^{-2}, \quad d \gg \xi_{2}$,

$\frac{I_{C}}{I_{0}}=\frac{\pi^{2} \xi_{2}}{16 \Gamma d} \frac{1-\left(4 / \vartheta \pi^{2}\right) \Psi[(1+\vartheta) / 2]-\Psi(1 / 2)}{\Psi\left[\left(1 / 2+\Gamma / \gamma_{B}^{2}\right)\right]-\Psi(1 / 2)}, \quad d \ll \xi_{2}$.

Note that at fixed $\Gamma \gg 1$ the reduction of the weak-link thickness deforms the CФR, shifting its maximum into the region $\varphi_{\max }<\pi / 2$.

\section{Depairing in superconducting electrodes by supercurrent}

It follows from Eqs. (47) and (48) that, if the N-layer thickness is small enough compared to $\xi_{2}$, the parameter range 


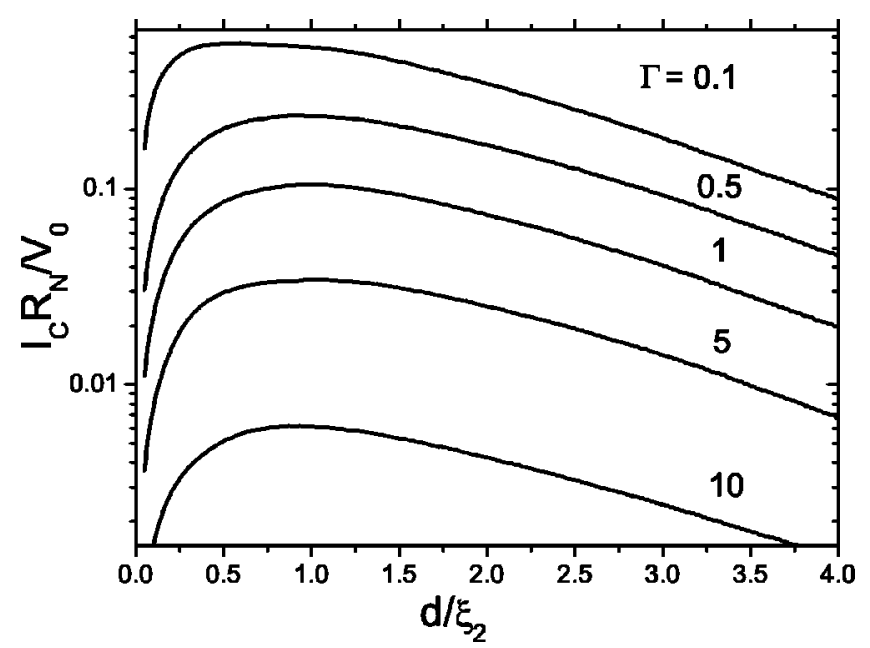

FIG. 9. Thickness dependence of the critical current in an SNS junction. Adapted from Ivanov et al., 1981.

$$
\gamma_{B}+\min \left\{1, d / \xi_{2}\right\} \ll \Gamma \ll \gamma_{B}+\max \left\{1, \xi_{2} / d\right\}
$$

exists with $\Gamma q \gg 1$ and $\Gamma p \ll 1$. Neglecting the term proportional to $\Gamma p$ in Eqs. (45) and (46), one arrives at

$$
\frac{I_{S}(\varphi)}{I_{0}}=\left\{\begin{array}{l}
(2 \Gamma q)^{-1} \cot (\varphi / 2), \quad \varphi \gg \frac{1}{\sqrt{\Gamma q}}, \\
\Gamma q \varphi\left[1-(\Gamma q \varphi / 2)^{2}\right], \quad 0 \leqslant \varphi \ll 1 \leqslant \frac{2}{\sqrt{3} \Gamma q}, \\
4 \Gamma q \varphi /\left[\Gamma q \varphi+\sqrt{4+(\Gamma q \varphi)^{2}}\right], \quad \frac{2}{\sqrt{3} \Gamma q} \leqslant \varphi \ll 1 .
\end{array}\right.
$$

The last two expressions in Eq. (60) merge asymptotically in the interval $(\Gamma q)^{-1 / 2} \ll \varphi \ll 1$. The supercurrent reaches its critical value at $\varphi=2(\sqrt{3} \Gamma q)^{-1}$ and is equal to the pair-breaking current of the superconducting electrodes. In this regime $I_{C} \propto\left(T_{c}-T\right)^{3 / 2}$.

Figure 9 shows the thickness dependence of $I_{C} R_{N}$ as numerically determined by Ivanov et al. $(1978,1981)$ for various values of parameter $\Gamma$. From the shape of this dependence one may conclude that, for reasonably small $\Gamma$, there is a broad range of parameters,

$$
\Gamma \ll \gamma_{B}+d / \xi_{2}, \quad \gamma_{B} \ll \gamma_{B}^{-1}+\xi_{2} / d, \quad d \leqslant \xi_{2},
$$

for which $I_{C} R_{N}$ is close to its maximum value $V_{0}$. Violation of the first inequality in Eq. (61) leads to a transition into the depairing current regime, with a linear decrease of $I_{C} R_{N}$ with decreasing $d$. The physical reason for the decrease of $I_{C} R_{N}$ with decreasing $d$ is that in this limit $I_{C}$ does not depend on $d$, while $R_{N}$ is proportional to $d$.

The violation of the last two inequalities in Eq. (61) results in a suppression of the critical current, with either the form of $I_{C} R_{N} \propto V_{0} / \gamma_{B}$ or exponential with thickness $I_{C} R_{N} \propto V_{0} \exp \left\{-d / \xi_{2}\right\}$. Since $\Gamma=\gamma / \sqrt{1-T / T_{c}}$, the smaller the $\gamma$, i.e., the higher the resistivity of the normal interlayer, the larger the interval of $d$ when the $I_{C} R_{N}$ product is large.

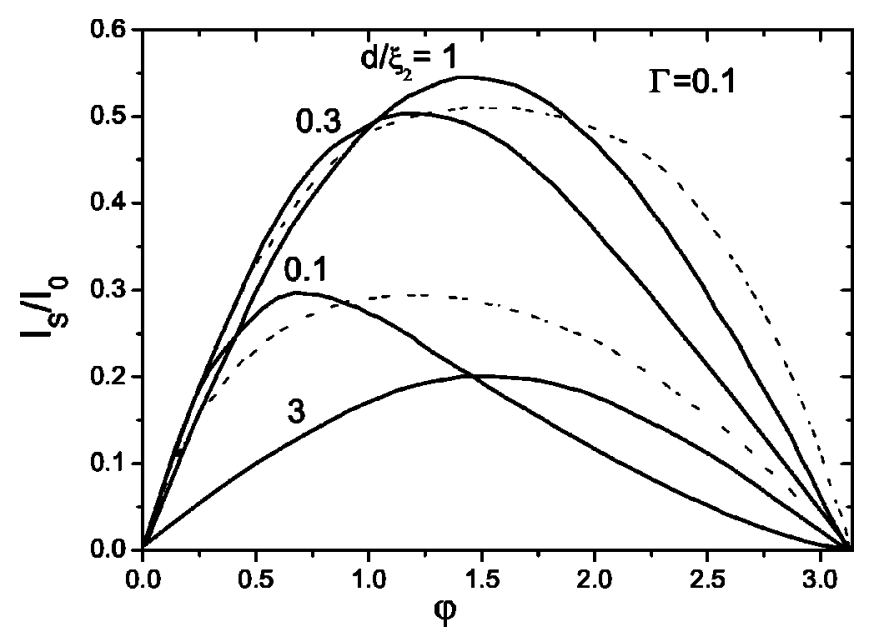

FIG. 10. The CФR for a SNS junction in the depairing regime. Adapted from Ivanov et al., 1981.

Note that increasing $T$ causes an increase of $\Gamma$ for fixed values of the parameters $\gamma_{B}, \gamma$, and $d / \xi_{2}$. There is therefore a crossover from the linear temperature dependence $I_{C} R_{N} \propto T_{c}-T$, predicted by the AslamazovLarkin theory, to the quadratic dependence $I_{C} R_{N} \propto\left(T_{c}\right.$ $-T)^{2}$, first suggested by de Gennes (1964).

\section{Nonlinear phase shift in the electrodes}

Figure 10 shows a set of $I_{S}(\varphi)$ curves calculated by Ivanov et al. $(1978,1981)$ for $\gamma_{B}=0, \Gamma=0.1$, and various ratios of $d / \xi_{2}$. It can be clearly seen that the reduction of $d / \xi_{2}$ deforms the CФR in such a way that their maximum shifts into the region $\varphi<\pi / 2$.

The CФR's shown in Fig. 10 by solid lines correspond to experimentally observed relations only at relatively low critical currents, when the kinetic inductance of the superconducting electrodes $L_{K}$ can be neglected in comparison with the Josephson-junction inductance $L_{J}$ $=\Phi_{0} / 2 \pi I_{C}$. In this case the phase difference across the junction increases linearly with the coordinate $x$ in the electrodes and is either negligibly small or can be subtracted by using the compensating measuring scheme

$$
\varphi(x)=\varphi(d / 2)+2 \frac{I_{S}(\varphi)}{I_{0}} \frac{\Delta_{0}^{2}}{\Delta^{2}(\infty)} \frac{x}{\xi(T)},
$$

resulting in $\varphi(\infty)=\varphi(d / 2)$. However, at sufficiently small $d$, namely, in the limit of large supercurrents, $L_{K}$ $\gg L_{J}$, the order parameter is suppressed by a supercurrent near the interfaces, and, in addition to a large linear team, there is also a nonlinear contribution to $\varphi$,

$$
\varphi(x)=\varphi(d / 2)+\int_{d / 2}^{\infty} \frac{I_{S}(\varphi)}{I_{0}}\left[\frac{\Delta_{0}^{2}}{\Delta^{2}(y)}\right] d y .
$$

The phase difference increases linearly with distance from the weak-link region only far from the interfaces, 


$$
\varphi(x)=\varphi(\infty)+2 \frac{I_{S}(\varphi)}{I_{0}} \frac{\Delta_{0}^{2}}{\Delta^{2}(\infty)} \frac{x}{\xi(T)} .
$$

Here $\varphi(\infty)$ is the phase difference that could be measured by a compensating measuring scheme and is given by the expression

$$
\varphi(\infty)=\varphi(d / 2)+\int_{d / 2}^{\infty} \frac{I_{S}(\varphi)}{I_{0}}\left[\frac{\Delta_{0}^{2}}{\Delta^{2}(y)}-\frac{\Delta_{0}^{2}}{\Delta^{2}(\infty)}\right] d y,
$$

which takes into account the effective increase in geometrical size of the weak-link region caused by destruction of the superconducting state in the electrodes in the vicinity of the $\mathrm{SN}$ interfaces.

The curves $I_{S}[\varphi(\infty)]$ (dashed lines in Fig. 10) also have maxima at $\varphi_{\max } \leqslant \pi / 2$. The smaller the $d / \xi_{2}$, the closer the system to the depairing limit and the larger the deviation of $\varphi_{\max }$ from $\pi / 2$.

5. Influence of the critical temperature of weak-link material on $I_{C} R_{N}$

The influence of the critical temperature of the weaklink material on $I_{C} R_{N}$ at $T \approx T_{c}$ has been discussed by Likharev and Yakobson (1975) and by Barone and Ovchinnikov (1979).

Likharev and Yakobson (1975) considered the situation in which the critical temperatures of both the weaklink material $T_{c}^{\prime}$ and the electrode material $T_{c}$ are close to the physical temperature, and consequently $T_{c}^{\prime} \approx T_{c}$. Assuming further that the pair potential at the interfaces is equal to its bulk value (the so-called rigid-boundary condition), Likharev and Yakobson (1975) reduced the problem of calculating the CФR to the solution of the Ginzburg-Landau equations in the interlayer. They found that if the junction is sufficiently short,

$$
d \ll \min \{\xi(T), \xi(T) / A\}, \quad A^{2}=\frac{T_{c}-T}{\left|T_{c}^{\prime}-T\right|},
$$

then in the first approximation on $d$ the CФR is given by

$$
\begin{aligned}
\frac{I_{S}(\varphi)}{I_{0}}= & {\left[\frac{\xi(T)}{d}+\left(\frac{1}{6}-\frac{A^{2}}{10}\right) \frac{d}{\xi(T)}\right] } \\
& \times \sin \varphi-\frac{1}{30} \frac{d}{\xi(T)} \sin 2 \varphi .
\end{aligned}
$$

According to Eq. (67), the corrections to a sinusoidal form of the CФR are small even for small $d$ of the order of $\xi(T)$ or $\xi(T) / A$. A further increase in $d$ results in effects that are different for $T>T_{c}^{\prime}$ and $T<T_{c}^{\prime}$.

For $T>T_{c}^{\prime}$, the weak-link material is in the normal state, superconducting correlations induced from the electrodes decay exponentially with increasing $d$, and the function $I_{S}(\varphi)$ remains almost sinusoidal. Maximum deviations from $\sin \varphi$ occur at $d \approx \xi(T)$, and, according to Eq. (67), they are of the order of a few percent.

For $T<T_{c}^{\prime}$, the weak-link material is in the superconducting state and, with increasing $d$, a transition from the Josephson effect to the depairing effect takes place. As a result, the critical current first decreases with $d$ (see

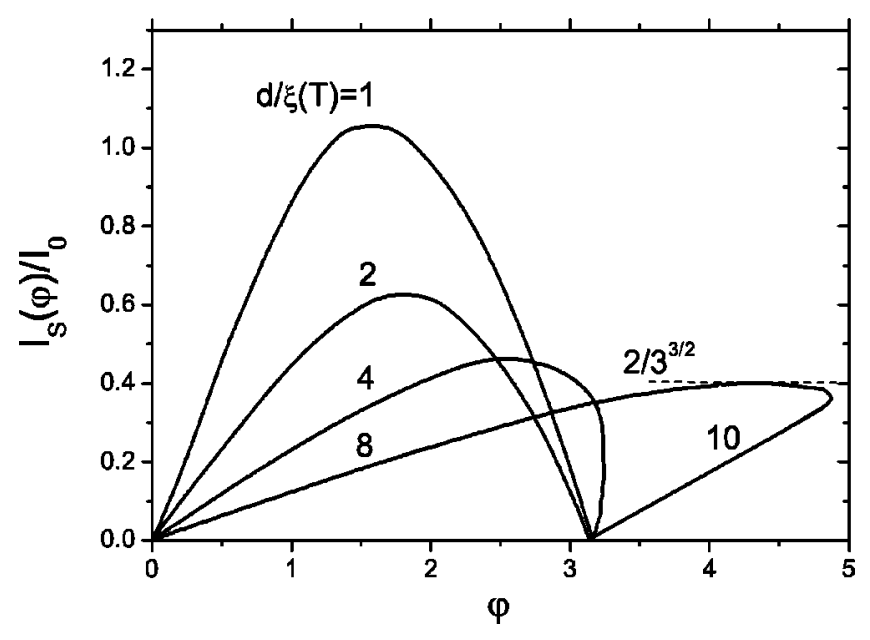

FIG. 11. Variation of the CФR with the length of a weak link at $T<T_{c}^{\prime}$. Adapted from Likharev and Yakobson, 1975.

Fig. 11) and then saturates at a constant value when $d$ exceeds approximately $10 \xi(T)$. Simultaneously, the shape of $I_{S}(\varphi)$ is radically changed. At $d \gtrsim 3.6 \xi(T)$, the $\mathrm{C} \Phi \mathrm{R}$ becomes multivalued, with a shape close to that given by the curve

$$
I_{S}(\varphi)=I_{C} \frac{\varphi \xi(T)}{d}\left[1-\left(\frac{\varphi \xi(T)}{d}\right)^{2}\right], \quad \frac{I_{C}}{I_{0}}=\frac{2}{3 \sqrt{3} A^{2}}
$$

The unstable reverse branch of the $I_{S}(\varphi)$ function corresponds to a sharp drop of $\Delta$ in the middle of the junction, i.e., to the nucleation of the phase-slip centers (see Ivlev and Kopnin, 1984; Sols and Ferrer, 1994).

Summarizing the discussion above, we conclude that, even at high temperatures, the CФR takes a variety of shapes. Deviations from $\sin (\varphi)$ depend on the relation between parameters characterizing either the properties of the weak-link material, like $d / \xi_{2}, T_{c}$, and $T_{c}^{\prime}$, or the influence of processes in the electrodes upon the junction's characteristics [e.g., the suppression parameters $\gamma$, $\gamma_{B}, T / T_{c}$, and $\left.d / \xi(T)\right]$.

To estimate the range of validity of the different regimes discussed above at arbitrary $T$, we shall subdivide the CФR problem into two parts: (1) influence of the processes in the weak link and (2) influence of the processes in the electrodes.

\section{B. Dirty limit, arbitrary temperature}

To calculate the CФR for dirty SNS junctions at arbitrary temperatures, the general approach is to solve the Usadel equations (A23) and (A24) in S and $\mathrm{S}^{\prime}$ and match them with the boundary conditions (A25) and (A26) at the interfaces. Far from the interfaces the solution tends to a uniform current-carrying superconducting state,

$$
\operatorname{Re} \Phi_{1}(\mp \infty)=\Phi_{\infty} \cos \varphi, \quad \operatorname{Im} \Phi_{1}(\mp \infty)=\mp \Phi_{\infty} \sin \varphi,
$$




$$
\begin{aligned}
& \Phi_{\infty}=\frac{\left|\Delta_{1}(\infty)\right|}{1+u^{2} \pi T_{c} / \sqrt{\omega^{2}+\Phi_{\infty}^{2}}}, \\
& \varphi=\varphi(\infty)-2 u x / \xi_{1},
\end{aligned}
$$

where $\varphi(\infty)$ is the asymptotic phase difference across the junction, $u=2 m v_{s} \xi_{1}, m$ is the electron mass, and $v_{s}$ is the superfluid velocity.

Note that, since the boundary conditions [Eqs. (A25) and (A26)] include the Matsubara frequency $\omega$, the phases of the $\Phi_{1}$ functions depend on $\omega$ and, in general, differ from the phase of the pair potential $\Delta$ at the $\mathrm{SN}$ interfaces, $\varphi( \pm d / 2)$. Therefore it is the value $\varphi(\infty)$ rather than $\varphi(d / 2)$ that can be measured experimentally, using a scheme that compensates for the part of Eq. (71) that is linear in $x$ (see also the related discussion in Sec. V.A).

We now consider several important limiting cases of the solution of the general problem.

\section{Rigid boundary conditions}

In this approximation it is assumed that suppression of superconductivity in the S layer is weak and the solution of the Usadel equations in the superconductor has the form

$$
\Phi_{1}\left( \pm \frac{d}{2}\right)=\Delta_{1} \exp \left\{ \pm \frac{\varphi}{2}\right\}, G_{S}=\frac{\omega}{\sqrt{\omega^{2}+\Delta_{1}^{2}}},
$$

where $\Delta_{1}$ is the modulus of the electrode order parameter. As discussed in Sec. III, these conditions are valid for the constriction weak-link geometry. The quantitative criteria for the validity of this approximation for planar SS'S sandwiches, where $S^{\prime}$ is a weak superconductor, and variable-thickness bridges will be discussed below for different parameter ranges.

In this approximation, the CФR depends on the reduced temperature $T / T_{c}$, the reduced weak-link thickness $d / \xi_{2}$, and the ratio $T_{c}^{\prime} / T_{c}$ (see Kulik and Omelyanchuk, 1975a; Likharev, 1976; Kupriyanov and Lukichev, 1981; Kupriyanov et al., 1981, 1982; Zaikin and Zharkov, 1981; Dubos et al., 2001).

In the limit of small interlayer thickness, $d \ll \xi_{2}$, the $\mathrm{KO}-1$ theory is valid and, as discussed in Sec. III.C, the C $\Phi \mathrm{R}$ is independent of $T_{c}^{\prime} / T_{c}$, and the maximum is $\varphi_{\text {max }} \geqslant \pi / 2$, tending to 1.86 at low $T$. Increasing $d$ leads to different effects, depending on the relationship between $T, T_{c}$, and $T_{c}^{\prime}$.

\section{a. Structures made from one material $\left(T_{c}=T_{c}^{\prime}\right)$}

The supercurrent across a junction consisting of bulk electrodes connected by a one-dimensional filament of the same material $\left(T_{c}=T_{c}^{\prime}\right)$ was analyzed by Kupriyanov and Lukichev (1981). The main effect predicted here is the crossover from Josephson to depairing effects with decreasing temperature for fixed values of the filament length. This is clearly demonstrated in Figs. 12-14

Figure 12 shows the CФR calculated numerically for structures with $d=10 \xi_{2}$ and $T / T_{c}=0.28,0.53$, and 0.8 .

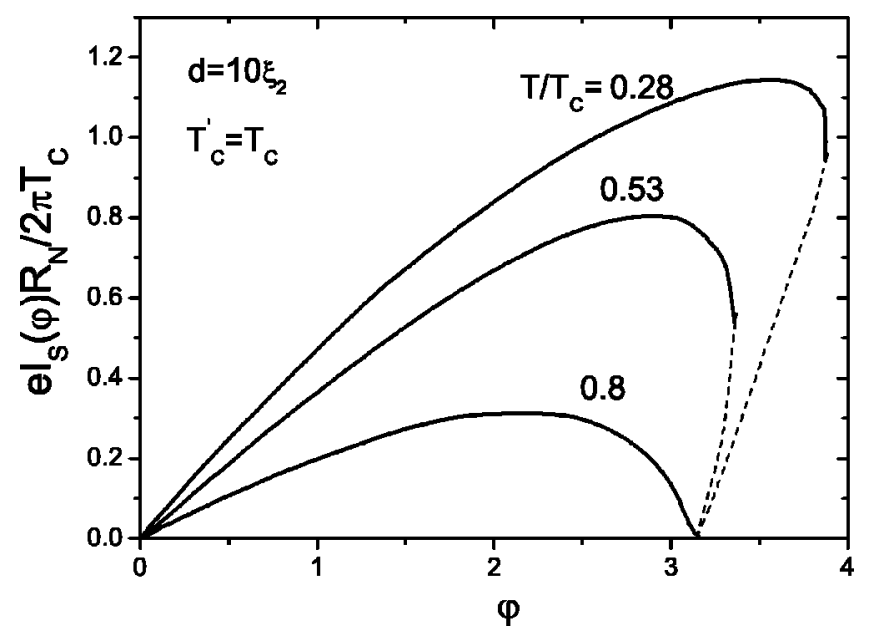

FIG. 12. Transformation from Josephson to depairing effect in a long SS'S junction with $T_{c}^{\prime}=T_{c}$. Adapted from Kupriyanov and Lukichev, 1981.

The deformation of the CФR from $\sin \varphi$ to a multivalued function of $\varphi$ at low $T$ can be clearly seen.

The physical reason for this transformation becomes clear if we take into account that the characteristic length over which the order parameter and Usadel functions vary is temperature dependent. For $T \lesssim T_{c}$, the properties of the junction depend on the relation between $d$ and the Ginzburg-Landau coherence length $\xi(T)$ given by Eq. (A29). In the vicinity of the critical temperature $\xi(T) \gg d$ and the C $\mathrm{CR}$ is sinusoidal, according to the Aslamazov-Larkin theory (Aslamazov and Larkin, 1969). With decreasing temperature, the coherence length $\xi(T)$ also decreases, which is equivalent to increasing the effective length of the structure, leading to a transition to the depairing regime, most clearly visible for $T \leqslant 0.5 T_{c}$. In this temperature range, the characteristic length of variation of the function $\Phi_{2}$ is close to $\xi_{2}$, and the effective length of the junction is large on this scale. As a result, for $T=0.28 T_{c}$ the Josephson critical current becomes rather close to the depairing current calculated for the same temperature by Kupriyanov and Lukichev (1980).

Figure 13 shows the temperature dependences of the $I_{C} R_{N}$ product calculated numerically for various $d / \xi_{2}$ ratios. The thin line in Fig. 13 is the $I_{C}(T) R_{N}$ dependence in the depairing limit (Kupriyanov and Lukichev, 1980); $R_{N}$ is the normal resistance calculated for $d$ $=10 \xi_{2} . I_{C}(T) R_{N}$ is smaller in this limit than in a junction of the same length $\left(d=10 \xi_{2}\right)$. The Josephson $I_{C}$ exceeds the depairing current because the electrodes additionally support superconductivity in the line.

For $T$ close to $T_{c}$, all of the curves have a similar temperature dependence: $I_{C} R_{N} \propto T_{c}-T$, as predicted by the Aslamazov-Larkin theory. (For the depairing limit, indicated by the thin line, $\left[I_{C}(T) R_{N} \propto\left(T_{c}-T\right)^{3 / 2}\right]$.) Deviations from this linear law depend on the length of the structure. The open circles in Fig. 13 mark the temperatures at which the CФR becomes multivalued. The locus of these positions determines the boundary between Josephson and depairing effects, shown in Fig. 13 by the 


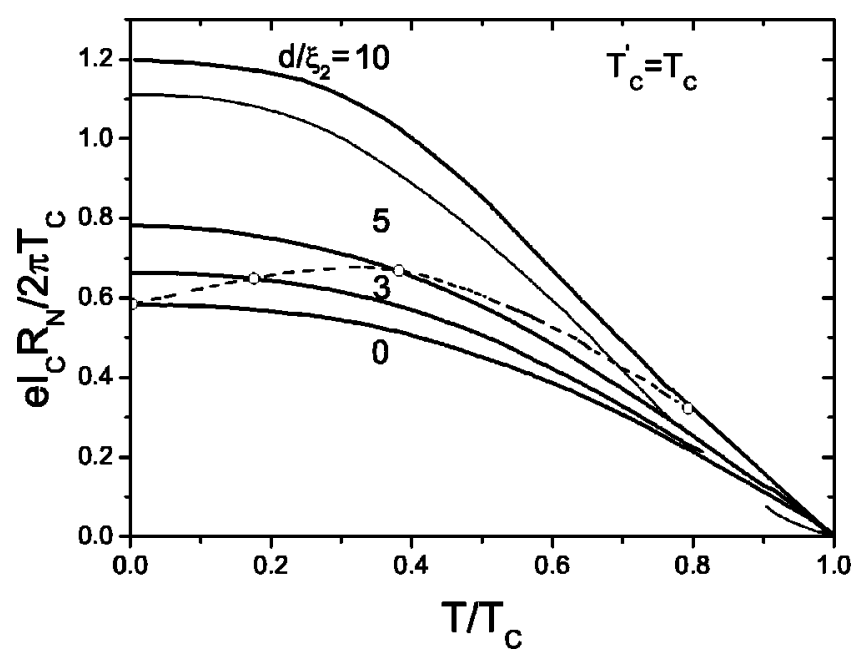

FIG. 13. Temperature dependence of the critical current in a SS'S junction with $T_{c}^{\prime}=T_{c}$. The open circles mark the temperatures when the C $\Phi \mathrm{R}$ starts to be multivalued. Adapted from Kupriyanov and Lukichev, 1981.

dash-dotted line. It is interesting that this curve has a maximum at $T \approx 0.5 T_{c}$, which corresponds to $d$ $\approx(4-6) \xi_{2}$, and that the maximum value of $I_{C} R_{N}$ in this region is even larger than $I_{C} R_{N}$ for a dirty point contact at $T=0$, predicted by the KO- 1 theory.

\section{b. $S S^{\prime} S$ junctions $\left(T_{c} \neq T_{c}^{\prime}\right)$}

The Josephson effect in SS'S junctions at arbitrary temperatures was analyzed by Kupriyanov et al. (1981, 1982) in the framework of the Usadel equations.

The results of this study are summarized in Fig. 14, which demonstrates the $I_{C} R_{N}$ products at $T_{c}^{\prime} / T_{c}=0.2$ for various ratios of $d / \xi_{2}$. As in Fig. 13, the open circles mark the temperatures at which the CФR starts to be multivalued at low $T$ when the depairing regime occurs.

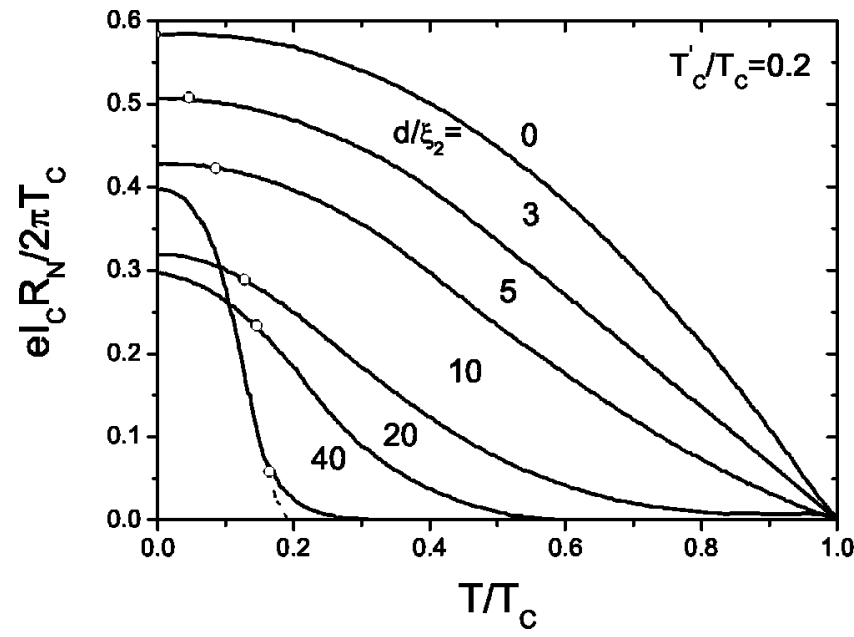

FIG. 14. Temperature dependence of the critical current in a SS'S junction with $T_{c}^{\prime}<T_{c}$. Adapted from Kupriyanov and Lukichev, 1981.
The smaller $T_{c}^{\prime} / T_{c}$ and $d / \xi_{2}$, the faster is the transformation from depairing to the Josephson effect with increasing $T$.

Note that at low temperatures, $T<T_{c}^{\prime}$, the $I_{C} R_{N}$ product first decreases with increasing $d$, but then increases (see the curve for $d / \xi_{2}$ in Fig. 14). The reason is that at $T<T_{c}^{\prime}$ the critical current $I_{C}$ is limited by the depairing effect and does not depend on $d$ for large $d$, while $R_{N}$ is proportional to $d$ and thus $I_{C} R_{N} \propto d$. Therefore these large $I_{C} R_{N}$ values do not correspond to the Josephson effect. The proportionality $I_{C} R_{N} \propto d$ is natural for the depairing of long one-dimensional superconducting filaments. It occurs in the parameter range corresponding to the left side from the line, which can be drawn via open circles in Fig. 14 and which marks the boundary between the depairing and the Josephson effects.

In the limit of large $d$ and $T \geqslant T_{c}^{\prime}$ the solution of the Usadel equations in the interlayer is a linear superposition of the functions $\widetilde{\Phi}_{2}(x)$ describing the penetration of superconductivity from the $\mathrm{S}$ electrode into the semiinfinite $S^{\prime}$,

$$
\begin{aligned}
\Phi_{2}(x)= & \widetilde{\Phi}_{2}(x-d / 2) \exp \{i \varphi / 2\} \\
& +\widetilde{\Phi}_{2}(x+d / 2) \exp \{-i \varphi / 2\} .
\end{aligned}
$$

Substitution of Eq. (73) into the expression for the supercurrent in Eq. (A27) leads to a sinusoidal CФR and exponential thickness dependence of the $I_{C} R_{N}$ product (Likharev, 1976):

$$
\frac{e I_{C} R_{N}}{2 \pi T_{c}}=V^{*} \frac{d}{\xi^{*}} \exp \left\{-\frac{d}{\xi^{*}}\right\}, \quad d \gg \xi^{*} .
$$

An exponential decrease in $I_{C} R_{N}$ as $d / \xi^{*} \rightarrow \infty$ is typical for SNS weak links (see de Gennes, 1964; Aslamazov and Larkin, 1969; Clarke, 1969), but the exact values of the prefactor $V^{*}$ and the effective decay length $\xi^{*}$ in Eq. (74) depend on $T_{c}^{\prime} / T_{c}$ and $T / T_{c}$. Analytic expressions for $V^{*}$ and $\xi^{*}$ for a symmetric SNS junction with $T_{c}^{\prime}=0$ were derived by Zaikin and Zharkov (1981):

$$
\begin{aligned}
& \xi^{*}=\xi_{2} \sqrt{\frac{T_{c}}{T}}, \\
& V^{*}=\frac{32 T \Delta^{2}}{T_{c}\left[\pi T+\Delta^{* 2}+\sqrt{2 \Delta^{*}\left(\pi T+\Delta^{* 2}\right)}\right]^{2}},
\end{aligned}
$$

where $\Delta^{*}=\sqrt{(\pi T)^{2}+\Delta^{2}}$. For arbitrary $T_{c}^{\prime}$ the determination of $V^{*}$ and $\xi^{*}$ is simplified by the existence of two different decay lengths for the function $\Phi_{2}$ and the pair potential $\Delta_{2}$ in the weak superconductor $\left(\mathrm{S}^{\prime}\right)$ material at low $T$. In the vicinity of the interfaces the decay is described by the nonlinear Usadel equations and occurs over distances of the order of $\xi_{2}$. Further decay of both $\Phi_{2}$ and $\Delta_{2}$ takes place on the scale $\xi^{*}>\xi_{2}$, with $\xi^{*}$ given to an accuracy better then $3 \%$ by the expression (Kupriyanov et al., 1982)

$$
\xi^{*}=\xi_{2} \sqrt{\frac{T_{c}}{T}\left[1+\frac{\pi^{2}}{4} \ln ^{-1} \frac{T}{T_{c}^{\prime}}\right]}, \quad T \geqslant T_{c}^{\prime} .
$$




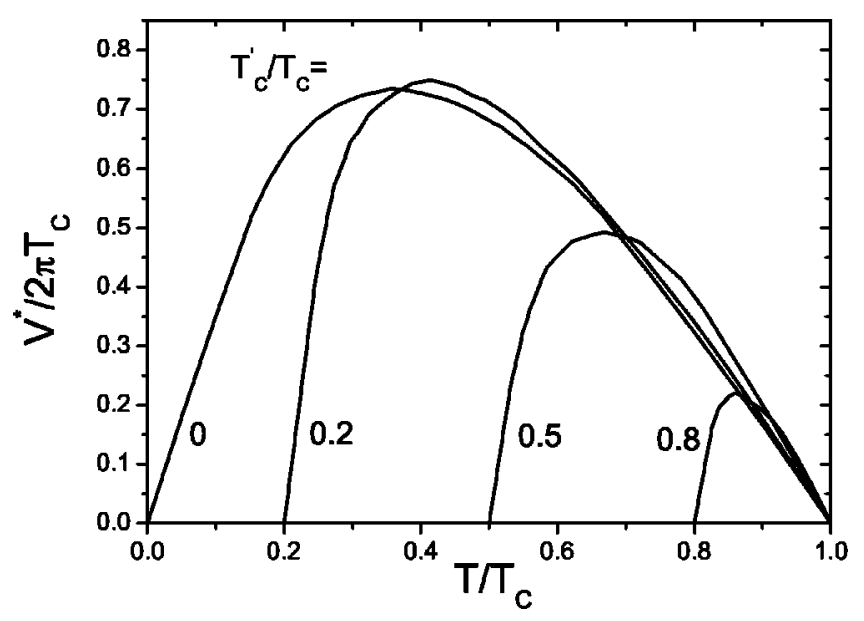

FIG. 15. Temperature dependence of the characteristic voltage $V^{*}$ for a SS'S junction for various $T_{c}^{\prime}$. Adapted from Kupriyanov et al., 1982.

The $V^{*}(T)$ dependencies calculated numerically by $\mathrm{Ku}-$ priyanov et al. (1982) are shown in Fig. 15. The curve for $T_{c}^{\prime}=0$ is close to the envelope of the curves corresponding to different values of $T_{c}^{\prime}$. This means that SS'S junctions with $T_{c}^{\prime} \neq 0$ can be described by Eq. (74) with the parameters $V^{*}$ and $\xi^{*}$ defined by Eqs. (75) and (76) over a wide temperature interval $T \geq T_{c}^{\prime}$.

It follows from Eq. (76) that, for a fixed $d$, there is a temperature interval with $T \approx T_{c}^{\prime}$ over which $\xi^{*} \geqslant d$ and Eq. (74) are violated. Instead, in the limit of $d \gg \xi_{2}$ the $I_{C} R_{N}$ product falls as $I_{C} R_{N} \propto\left(\xi_{2} / d\right)^{2}$. The CФR in this limit is close to that in the KO-1 theory.

The case of SNS junctions with $T_{c}^{\prime}=0$ was first analyzed by Likharev (1976). More extensive calculations were performed by Dubos et al. (2001), who demonstrated that, in a long-junction limit $\left(d \gg \xi_{2}\right)$ at $T=0$,

$$
e I_{C} R_{N}=20.64 \pi T_{c}\left[\frac{\xi_{2}}{d}\right]^{2}=10.82 E_{T h} .
$$

Here $E_{T h}=\hbar D / d^{2}$ is the Thouless energy, the natural scale for the proximity effect, which provides the magnitude of the induced energy gap in a diffusive normal metal attached to a superconductor (Golubov and $\mathrm{Ku}-$ priyanov, 1988; Aminov et al., 1996; Courtois et al., 1996; Melsen et al., 1996; Zhou et al., 1998; Belzig et al., 1999; Pannetier and Courtois, 2000). In contrast to the pair potential $\Delta$ set by the interactions in the superconducting electrodes, the Thouless energy $E_{T h}=\hbar D / d^{2}$ is a single-electron quantity: it provides the diffusion rate for a single electron across a sample of length $d$. The condition of a long junction length, $d \gg \xi_{2}$, is equivalent to $\Delta \gg E_{T h}$.

The CФR in a long junction, Eq. (77), has $\varphi_{\max }$ $\approx 1.27 \pi / 2$ (for comparison, the KO-1 theory valid for $d$ $\lesssim \xi_{2}$ provides $\varphi_{\max } \approx 1.25 \pi / 2$ ).

Studies of the temperature dependences of $I_{C} R_{N}$ for SNS junctions with $T_{c}^{\prime}=0$ are summarized by Delin and Kleinsasser (1996) and Dubos et al. (2001).

The Josephson effect can take place in SNS weak links even if the effective electron-phonon interaction in the $\mathrm{N}$ layer is repulsive $\left(\lambda_{N}<0\right)$. The repulsion suppresses the ability of the weak-link material to carry supercurrent and leads to a decrease in $I_{C} R_{N}$. This effect was analyzed by Kupriyanov et al. (1982), who showed that the main influence of a negative $\lambda_{N}$ is a decrease of the decay length from $\xi^{*}=\xi_{2}$ for $\lambda_{N}=0$ to $\xi^{*} \approx 0.65 \xi_{2}$ in the limit of strong repulsion. Even for $\left|\lambda_{N}\right|=0.9 \lambda_{S}$ (where $\lambda_{S}$ is the BCS coupling constant in the superconducting electrodes) the temperature dependencies of $I_{C} R_{N}$ for $d / \xi_{2} \leqslant 5$ differ only slightly from those for $\lambda_{N}$ $=0$.

Besides negative $\lambda_{N}$, there are several other physical mechanisms that lead to suppression of the $I_{C} R_{N}$ product and a deformation of the shape of the C $\Phi R$ in weak links. They are

- suppression of superconductivity in the electrodes due to the proximity effect at $S^{\prime} S$ interfaces,

- depairing in the electrodes by the current across the junction,

- potential barriers at $S^{\prime} S$ interfaces.

Fortunately, the first mechanism is most effective for $d>\xi_{2}$, while the second is important in the opposite limit, $d<\xi_{2}$. This allows for an independent analysis of the influences of these factors on weak-link properties. The influence of the transparency of S'S interfaces on SINIS junctions will be discussed in Sec. VI.

\section{SNS junction at arbitrary $T$ : Depairing due to the proximity} effect

The proximity of normal and superconducting materials results not only in the penetration of superconducting correlations into the normal metal, as studied above for rigid boundary conditions. It also causes quasiparticle excitations, which can diffuse into the superconductor, resulting in suppression of superconductivity near the interface. The influence of this effect on long one-dimensional planar-type SNS junctions $\left(d>\xi_{2}\right)$ was analyzed by Kupriyanov and Lukichev (1982). They supposed that both interfaces are transparent and that $T_{c}^{\prime}$ $=0$. Their calculations lead to a sinusoidal CФR and an exponential dependence of $I_{C} R_{N}$. Equation (74), with $\xi^{*}=\xi_{2}$ and a temperature-dependent $V^{*}$, is shown in Fig. 16 for various values of $\gamma$, defined in Eq. (A21).

Clearly, for $\gamma \lesssim 0.1$ the $I_{C} R_{N}$ values are close to those calculated using rigid boundary conditions, Eq. (75), over a broad temperature range. An increase of $\gamma$ leads to suppression of $I_{C} R_{N}$. If normal metals like $\mathrm{Au}, \mathrm{Ag}$, $\mathrm{Cu}$ are used, then $\gamma \geqslant 1$, and the magnitude of the suppression exceeds two orders of magnitude at $T \approx 0.5 T_{c}$.

Thus to get high values of $I_{C} R_{N}$ it is necessary to use highly resistive weak-link materials.

In variable-thickness bridges composed of two superconducting-banks placed on the top of a thin normal film of thickness $d_{n}$, the situation is close to that described above (see Golubov et al., 1983; Kupriyanov, 1989) and the $I_{C} R_{N}$ product is weakly suppressed if $\gamma$ $\leqslant 0.3 \max \left\{1, \xi_{2} / d_{n}\right\}$. 


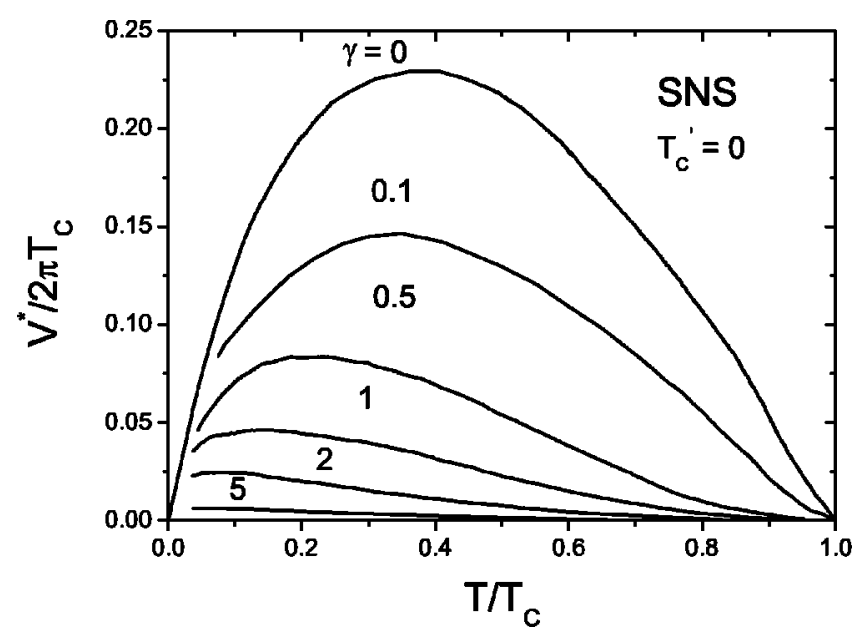

FIG. 16. Temperature dependence of the critical voltage for a $\mathrm{SS}^{\prime} \mathrm{S}$ junction for various values of the proximity-effect parameter $\gamma$. Adapted from Kupriyanov and Lukichev, 1982.

\section{SS'S junction at arbitrary $T$ : Depairing by the supercurrent}

The suppression of superconductivity in electrodes by a supercurrent across an $\mathrm{SS}^{\prime} \mathrm{S}$ junction may become essential if

$$
d \ll \xi_{2}
$$

and the transparency of $\mathrm{S}^{\prime} \mathrm{S}$ interfaces is high. This situation has been studied by Zubkov and Kupriyanov (1983) within the framework of the Usadel equation. Based on Eq. (78) they reduced the problem to the solution of Eqs. (A23) and (A24) with the boundary conditions at the $\mathrm{S}^{\prime} \mathrm{S}$ interface

$$
\begin{aligned}
& \xi_{1} G_{1} \partial_{x} \operatorname{Im} \Phi_{1}=2 \omega \gamma_{J} \arctan \left[\frac{G_{1} \operatorname{Im} \Phi_{1}}{\omega}\right], \\
& G_{1}=\frac{\omega}{\sqrt{\omega^{2}+\operatorname{Re} \Phi_{1}^{2}}}, \quad \gamma_{J}=\gamma \frac{\xi_{2}}{d}
\end{aligned}
$$

with the conditions given by Eqs. (69)-(71) in the bulk.

Figure 17 shows the CФR calculated for $T=0.1 T_{c}$ by Zubkov and Kupriyanov (1983). Here, $\varphi(\infty)$ is the asymptotic phase difference defined by Eq. (71). At $\gamma_{J}$ $=0$ the KO- 1 theory is valid and $\varphi_{\max }$ is $\approx 1.25 \pi / 2$, as shown in Fig. 17. An increase of $\gamma_{J}$ makes $I_{S}[\varphi(\infty)]$ close to the $\sin (\varphi)$ form at $\gamma_{J} \approx 2.5$ and shifts $\varphi_{\max }$ to a value smaller than $\pi / 2$ for larger $\gamma_{J}$. At high temperatures in the absence of the depairing effect $\left(\gamma_{J}=0\right.$, rigid boundary conditions), the shape of $I_{S}(\varphi)$ is close to the sinusoidal form. In complete agreement with the situation discussed above in Sec. V, an increase of $\gamma_{J}$ leads to deformation of the shape of $I_{S}[\varphi(\infty)]$ so that the position of its maximum shifts to $\varphi_{\max }<\pi / 2$.

Numerical calculations made by Zubkov and Kupriyanov (1983) have shown that at

$$
\gamma_{J} \lesssim 0.1
$$

there is a large temperature interval where the suppression of superconductivity in the $\mathrm{S}$ electrodes is small. At larger $\gamma_{J}$ and $T \lesssim 0.4 T_{c}$ the ratio decays exponentially with $\gamma_{J}$,

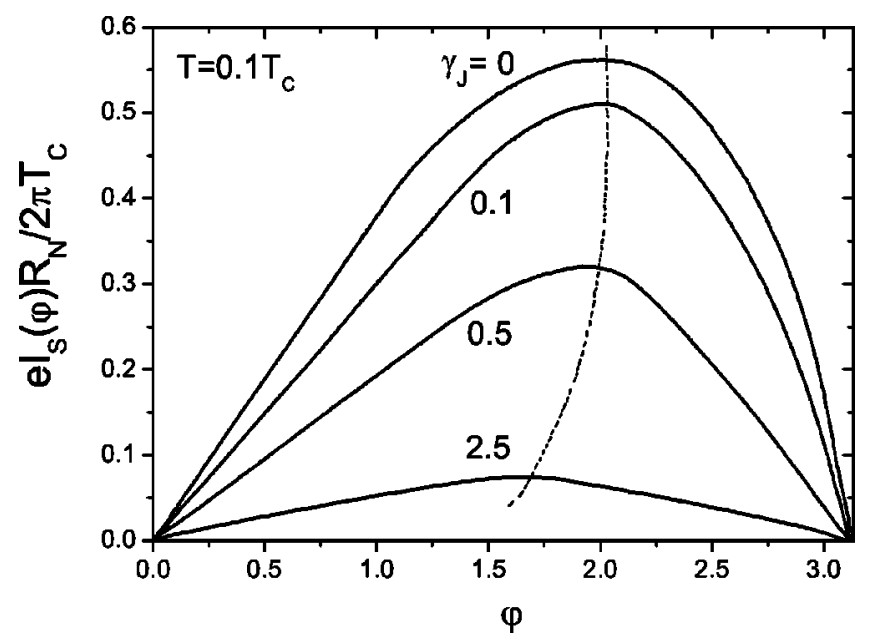

FIG. 17. The CФR in a SS'S junction for various values of the depairing current parameter $\gamma_{J}$. Adapted from Zubkov and Kupriyanov, 1983.

$$
\frac{I_{C} R_{N}\left(T, \gamma_{J}\right)}{I_{C} R_{N}(T, 0)} \approx 0.8 \exp \left\{-\frac{\gamma_{J}}{1.45}\right\}, \quad \gamma_{J} \gtrsim 0.2 .
$$

At $T \approx T_{c}$ an additional temperature-dependent factor should be taken into account and the parameter $\Gamma_{J}$ $=\gamma_{J} / \sqrt{1-T / T_{c}}$ should be used instead of $\gamma_{J}$. This means that under fixed $\gamma_{J}$ there will be a transition to the depairing regime similar to that discussed above.

We can conclude that the processes in the electrodes are not essential if

$$
\gamma \leqslant 0.1\left(\gamma_{B}+\min \left\{1, d / \xi_{2}\right\}\right) \sqrt{1-T / T_{c}} .
$$

The violation of this inequality leads to a suppression of the $I_{C} R_{N}$ product and deformation of the shape of $I_{S}(\varphi)$, calculated on the base of rigid boundary conditions. The deviations from Eq. (83) make the dependence $I_{S}[\varphi(\infty)]$ closer to the sinusoidal form. In some cases $\varphi_{\max }<\pi / 2$ may be expected. It is important that, due to nonlinear effects in the electrodes, the phase difference be considered in the sense of Eq. (71). Strictly speaking, $\varphi(\infty) \approx \varphi$ only if the condition

$$
\gamma \leqslant 0.1\left(\gamma_{B}+d / \xi_{2}\right) \sqrt{1-T / T_{c}}
$$

is not violated.

\section{Clean SNS junctions}

Now we shall briefly examine the properties of clean weak links in which the mean free path of an electron $\ell_{2}$ is larger than the distance between the superconducting electrodes $d$ and weak-link coherence length $\xi_{02}$ $=v_{F 2} / 2 \pi T_{c}$ and in which the condition of the clean limit $\ell_{1} \gg \xi_{01}=v_{F 1} / 2 \pi T_{c}$ is also fulfilled in the superconducting electrodes.

The Josephson coupling and the shape of the CФR in these structures have been studied by Kulik (1969), Ishii (1970), Svidzinskii et al. (1971), and Bardeen and Johnson (1972). In these models it was supposed that the superconductor and normal metal had the same 


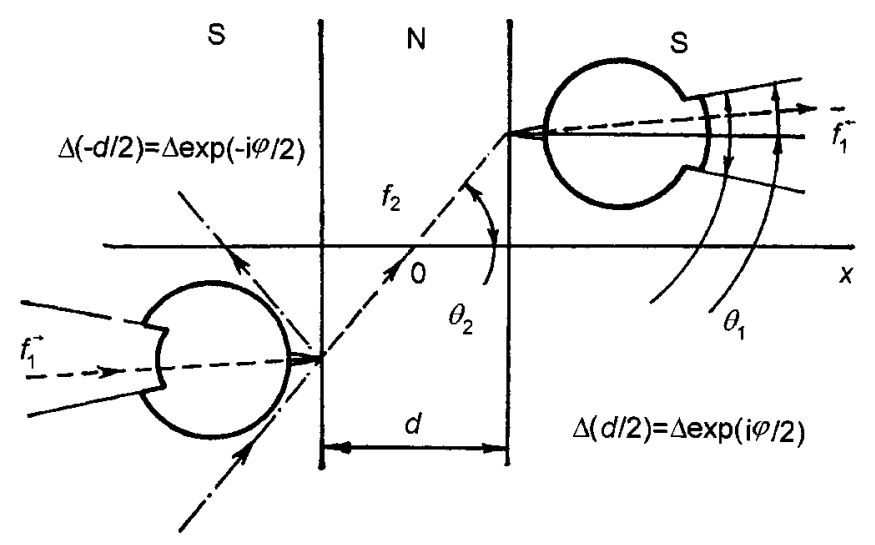

FIG. 18. Processes of current transport in a clean SNS junction.

transport parameters, that $T_{c}^{\prime}=0$ in the normal material, that the distance between superconducting electrodes was large $\left(d \gg \xi_{02}\right)$, and that $T \ll T_{c}$. The suppression of superconductivity in the electrodes was neglected and rigid boundary conditions were used for the calculation of $I_{S}(\varphi)$.

Zaikin and Zharkov (1983) found that under the above conditions the pair potential was suppressed at the interface and its magnitude at $T \ll T_{c}$ was twice as small as in the bulk. Thus the rigid boundary conditions were not strictly valid.

On the other hand, it was shown by Kupriyanov (1981) that the rigid boundary conditions were still applicable if there was a large difference between Fermi velocities of the metals, namely,

$$
v_{F 2} \ll v_{F 1} \text {. }
$$

Under this condition, only a small number of electrons from the $\mathrm{S}$ metals moving almost perpendicular to the $\mathrm{SN}$ interface could penetrate from $\mathrm{S}$ into $\mathrm{N}$ and finally from $\mathrm{N}$ into the second electrode, thus providing a Josephson coupling in the structure (see Fig. 18). Smooth transparent interfaces were assumed in this model. The electrons in $\mathrm{S}$ with the momentum component parallel to the interface larger than $v_{F 2}$ were reflected back into $\mathrm{S}$. This meant that, exactly as in the KO-2 model (Kulik and Omelyanchuk, 1977, 1978), the electron distribution function in the superconductor momentum space deviated from its bulk value only in the small-angle range

$$
\left|\theta_{1}\right| \leqslant v_{F 2} / v_{F 1},
$$

where $\theta_{1}$ is the angle between the $x$ axis and direction of $\mathbf{v}_{F 1}$. Therefore, to solve the Eilenberger equations (A6) and (A7), one may assume that the pair potential $\Delta$ and the isotropic parts of functions $\left\langle f_{1}\right\rangle,\left\langle f_{1}^{+}\right\rangle$, and $\left\langle g_{1}\right\rangle$ in the electrodes are spatially homogeneous and equal to their bulk values, which in the symmetrical case are given by

$$
\begin{aligned}
& \left\langle f_{1}\left( \pm \frac{d}{2}\right)\right\rangle=\frac{\Delta\left( \pm \frac{d}{2}\right)}{E_{0}}, \\
& \left\langle f_{1}^{+}\left( \pm \frac{d}{2}\right)\right\rangle=\frac{\Delta^{*}\left( \pm \frac{d}{2}\right)}{E_{0}},
\end{aligned}
$$

$$
\begin{aligned}
& \left\langle g_{1}\right\rangle=\frac{\omega}{E_{0}}, \\
& E_{0}=\sqrt{\omega^{2}+\Delta^{2}}, \quad \Delta\left( \pm \frac{d}{2}\right)=\Delta_{1} \exp \left\{ \pm i \frac{\varphi}{2}\right\},
\end{aligned}
$$

where $\Delta_{1}$ is the magnitude of the pair potential in the superconducting electrodes. As a result, Eq. (A6) is reduced to a linear differential equation similar to that solved in the KO-2 model. The solutions for the outgoing $(\rightarrow)$ and the incoming $(\leftarrow)$ trajectories have the form

$$
\begin{aligned}
& f_{1}^{\leftrightarrows}=\frac{\Delta}{E_{0}}+f^{\leftrightarrows} \exp \left\{-\frac{|d / 2-x|}{\xi^{*} \cos \theta_{1}}\right\}, \\
& g_{1}^{\leftrightarrows}=\frac{\omega}{\xi}+g^{\leftrightarrows} \exp \left\{-\frac{|d / 2-x|}{\xi^{*} \cos \theta_{1}}\right\},
\end{aligned}
$$

where

$$
\begin{aligned}
f_{1} & =g^{\rightarrow} \frac{\Delta}{\omega-E_{0}}, \quad f_{1}^{\leftarrow}=g^{\star} \frac{\Delta}{\omega+E_{0}}, \\
\xi^{*} & =\xi_{01}\left[\frac{E_{0}}{\pi T_{c}}+\frac{\xi_{01}}{\ell_{1}}\right]^{-1} .
\end{aligned}
$$

In the range of angles $\theta_{1} \geqslant\left(v_{F 2} / v_{F 1}\right) \sin \theta_{2}$, where $\theta_{2}$ is the angle between the $x$ axis and the direction of electron Fermi momentum $\mathbf{v}_{F 2}$ in the $\mathrm{N}$ metal, the functions $f_{1}=\left\langle f_{1}\right\rangle$ and $g_{1}=\left\langle g_{1}\right\rangle$ are equal to their bulk values as given in Eqs. (87) and (88).

In the normal metal $\Delta_{2}=0$, and the solutions of Eq. (A6) in the limit $\ell_{1} \rightarrow \infty$ have the simple form

$$
f_{2}=C \exp \left\{-\frac{x}{\xi_{02} \cos \theta_{2}} \frac{\omega}{\pi T_{c}}\right\}, \quad g_{2}=\sqrt{1-C C^{*}} .
$$

The integration constants $C, g^{\rightarrow}$, and $g^{\sharp}$ are defined by requiring that the solutions $(89)-(92)$ be continuous at $x= \pm d / 2$. This provides the supercurrent (see also Zagoskin, 1997)

$I_{S}(\varphi)$

$$
=\sum_{\omega>0} \int_{0}^{1} \frac{4 \pi T e N_{2} v_{F 2} \Delta_{1}^{2} \sin (\varphi) z d z}{\left(\omega^{2}+E_{0}^{2}\right) \cosh \varkappa+2 \omega E_{0} \sinh \varkappa+\Delta_{1}^{2} \cos (\varphi)},
$$

$$
\varkappa=\frac{d}{\xi_{02} z} \frac{\omega}{\pi T_{c}}, \quad z=\cos \theta_{2} .
$$

Equation (93) was first derived by Svidzinskii et al. (1971). The spectral current can be obtained by the analytical continuation $\omega=-i E+0$ in Eq. (93) and yields the equation for an Andreev bound state in a clean SNS contact. For $d \gg \xi_{0 T}$, the energies of the low-lying bound states, $E_{n} \ll \Delta_{1}$, are given by the well-known simple expression (Kulik, 1969)

$$
E_{n}=\frac{\hbar v_{F} z}{d} \pi\left(n+\frac{1}{2}-\frac{\varphi}{2}\right) \text {. }
$$

Kupriyanov (1981) generalized Eq. (93) to the case of different superconducting electrodes. A more general 
expression, taking into account the full asymmetry in the structure, has been obtained by Galaktionov and Zaikin (2002).

In the limit of small N-layer thickness, $d \ll \xi_{02}$, the sum in Eq. (93) converges at $\omega$ under which $x \ll 1$. Substitution of $\cosh x \approx 1$ and $\sinh x \approx 0$ in Eq. (93) provides the CФR of the KO-2 model.

In the opposite case of $d \gg \xi_{0 T}=\xi_{02} T_{c} / T$ the CФR is sinusoidal with an exponentially small critical current,

$$
I_{S}(\varphi)=\frac{8 \pi T e N_{2} v_{F 2} \Delta_{1}^{2} \sin (\varphi)}{\left[\pi T+\sqrt{(\pi T)^{2}+\Delta_{1}^{2}}\right]^{2}} \frac{\xi_{0 T}}{d} \exp \left\{-\frac{d}{\xi_{0 T}}\right\} .
$$

For a long SNS junction $\left(\xi_{02} \ll d \ll \xi_{0 T}\right)$ at low $T$ one can transform the sum over $\omega$ in Eq. (93) to the integral

$$
2 \pi \sum(\cdots) \rightarrow \int(\cdots) d \omega \rightarrow \int(\cdots) \Delta_{1} \cosh u d u,
$$

where $\omega=\Delta \sinh u$. The main contribution to the integral comes from small $u$ and the result is (Ishii, 1970)

$$
I_{S}(\varphi)=\frac{1}{2} e N_{2} v_{2} \Delta_{1} \sum_{k=1}^{\infty}(-1)^{k-1} \frac{\sin k \varphi}{k} .
$$

The Fourier series, Eq. (96), determines the periodic $I_{S}(\varphi)$ function in the form

$$
I_{S}(\varphi)=e N_{2} v_{2} \Delta_{1}\left\{\frac{\varphi}{2}-\pi\left[\frac{\varphi}{2 \pi}+\frac{1}{2}\right]\right\},
$$

where $[x]$ is the integer part of $x$. Details of these calculations can be found in the textbook by Svidzinskii (1982).

Thus, in clean SNS junctions, the CФR transforms from the sinusoidal form at $T \approx T_{c}$ to the saw-toothed curve of Eq. (97) at low $T$ if the suppression of superconductivity by current or proximity effect is negligibly small and rigid boundary conditions are valid.

The range over which rigid boundary conditions are valid at a clean NS interface was analyzed by Kupriyanov and Lukichev (1988b). They considered thick ( $d$ $\gg \xi_{0 T}$ ) SNS junctions with fully transparent interfaces under arbitrary ratio between Fermi velocities of normal and superconducting materials. Starting with the analytical solution of the Eilenberger equations for a normal metal (92), they derived the general expression for the supercurrent

$$
\begin{aligned}
I_{S}(\varphi)= & \frac{8 \pi T e N_{2} v_{F 2} \Delta_{1} \sin (\varphi)}{\gamma_{C}^{2}\left[\pi T+\sqrt{(\pi T)^{2}+\Delta_{1}^{2}}\right]} \int_{0}^{\theta_{p}} \Phi_{+}(\theta) \\
& \times \exp \left\{-\frac{d}{\xi_{0 T} \sqrt{1-\gamma_{C}^{-2} \sin ^{2}(\theta)}}\right\} \sin (\theta) \cos (\theta) d \theta \\
\gamma_{C}= & \frac{v_{F 2}}{v_{F 1}}, \quad \Phi_{+}(\theta)=\frac{1}{2}\left[f_{1}\left(\frac{d}{2}, \theta\right)+f_{1}^{+}\left(\frac{d}{2}, \theta\right)\right] .
\end{aligned}
$$

Here $\theta_{p}=\pi / 2$, if $\gamma_{C} \geqslant 1$, and $\theta_{p}=\arcsin \gamma_{C}$, if $\gamma_{C} \leqslant 1$, and $f_{1}(d / 2, \theta)$ is the Eilenberger function on the supercon- ducting side of the NS interface. Thus the problem is reduced to a calculation of the function $\Phi_{+}(\theta)$ by a numerical solution of the Eilenberger equations in the superconducting electrodes.

In the limit of small $\gamma_{C} \ll 1$, the integration in Eq. (98) takes place only in a very small cone. As discussed above, the supercurrent is defined by the function $\Phi_{+}(\theta)=\Delta_{1} /\left[\pi T+\sqrt{(\pi T)^{2}+\Delta_{1}^{2}}\right]$ on the trajectory perpendicular to the interface, and Eq. (98) is reduced to Eq. (95).

In the opposite case of $\gamma_{C} \gg 1$, the supercurrent falls as $\gamma_{C}^{-2}$ and has a slightly different dependence on $d$. Note that the function $\Phi_{+}(\theta)$ depends on $\gamma_{C}$ only for $\gamma_{C}$ $\leqslant 1$. Moreover, the suppression of $\Phi_{+}(\theta)$ with an increase of $\gamma_{C}$ is essentially weaker than in the dirty limit, when for $\gamma \approx 1$ the $I_{C} R_{N}$ is reduced by two orders of magnitude (see Sec. V.B.2).

As a result, for $\gamma_{C} \leqslant 1$ and over a wide temperature range $T \leqq 0.8 T_{c}$ the suppression of superconductivity in the electrodes does not essentially modify the results obtained in the framework of the rigid boundary conditions.

Let us now consider briefly the opposite limiting case of $T_{c}^{\prime}=T_{c}$, when two superconductors are connected by a narrow wire of length $d$ made from the same material. In a short contact, $d \ll \xi_{0 T}, I_{S}(\varphi)$ is described by the KO-2 model. However, strong modifications take place as one moves to the opposite case, $d \gg \xi_{0 T}$. Starting from a critical value $d_{C} \sim \xi_{0 T}$, the function $I_{S}(\varphi)$ becomes multivalued (Kulik and Omelyanchuk, 1975b; Likharev, 1979; Martin-Rodero et al., 1994; Sols and Ferrer, 1994). This situation corresponds to the appearance of the solution with a phase-slip center inside the contact and is similar to that discussed above in Sec. V.B.3 for the dirty limit.

Interesting peculiarities in the CФR of ballistic and symmetrically stacked double SNSNS junctions were predicted by Chang and Chu (1997).

\section{DOUBLE-BARRIER SINIS JUNCTIONS}

Double-barrier junctions have recently attracted considerable interest both in experiments and in theory. A supercurrent in a tunnel SIS contact depends linearly on the barrier transparency, since Cooper pairs tunnel coherently (Josephson, 1962). Supercurrent transport in an SINIS structure, having two barriers, is of a more complicated nature. It is well known from the theory of Blonder, Tinkham, and Klapwijk (1982) that the subgap resistance of a ballistic SIN junction has a quadratic dependence on the transparency of the interface, since Andreev reflection is a two-particle process. The disorder in a normal region enhances the Andreev current due to opening of some fraction of the tunneling channels, and the resistance has a linear dependence on the transparency. This effect is known as reflectionless tunneling in SIN junctions [see Volkov et al. (1993), Nazarov (1994b), and further references in the review article by Beenakker (1997)]. Interestingly, tunnel channels may be 
opened in a ballistic $N I_{1} N I_{2} S$ junction, as well, by placing a second tunnel barrier [Melsen and Beenakker (1994)]. Therefore it is important to understand how barrier transparency and disorder influence coherence in SINIS junctions and what the corresponding modifications of the CФR are.

Coherent effects in SINIS junctions are of significant practical importance. Recent experiments have demonstrated the possibility of engineering Josephson junctions with desired properties using existing multilayered techniques. As already mentioned in Sec. II.B, the present interest in SINIS junctions is related to their possible application in superconducting electronics. References to experiments are given in Sec. II.B.

An overview of the early experimental work on supercurrent flow in superconductor-semiconductorsuperconductor junctions was given by van Huffelen et al. (1993). Carrier transport through these structures was found to be dominated by interfaces; therefore they are of the SINIS type. Several types of weak links using various semiconducting materials that carry large supercurrents were reported by Klapwijk et al. (1989) and Kleinsasser et al. (1989). The operation of a superconducting field-effect transistor, with a gate electrode controlling the supercurrent, has been demonstrated. More recently, structures using two-dimensional electron gas (2DEG) as a weak-link material were successfully fabricated and investigated by Marsh et al. (1994), Takayanagi et al. (1995a), Mur et al. (1996), Chrestin et al. (1997, 1999), Heida et al. (1999), Lehnert et al. (1999), Neurohr et al. (1999), Grajcar, Ebel, et al. (2002), and Schäpers et al. (2003). A qubit device based on manipulating Andreev bound states in SINIS junctions was recently proposed by Shafranjuk et al. (2002).

A theory of long SINIS junctions with low barrier transparency was developed by Aslamazov et al. (1968; see also discussion in the previous section). Nikolić et al. (2002) recently reported on a theoretical study of double-screened dipole barrier SINIS junctions, which are of relevance to high- $T_{c}$ superconducting grainboundary junctions.

In this section, recent developments in the theory of SINIS structures with low-transparency barriers are discussed. We shall concentrate on SINIS junctions with thin metallic interlayers, which are of the most practical importance.

\section{A. SINIS junctions, clean limit}

\section{The general case}

Supercurrent in ballistic SINIS structures is controlled by an interplay between Andreev bound states and transmission (Breit-Wigner) resonances. Much theoretical work has concentrated on studying resonant supercurrents in low-dimensional structures, in particular in S-2DEG-S (where 2DEG is a two-dimensional electron gas).
Supercurrents in such structures were calculated using various theoretical approaches. ${ }^{8}$ Much of this work was devoted to the physics of supercurrent flow through Breit-Wigner resonances in ballistic structures. The common conclusion from these studies is that the physics of supercurrent via a single Breit-Wigner resonance is similar to that of resonant tunneling via a localized state in SIS tunnel junctions, discussed above in Sec. III. The CФR depends on the width of the resonance and its position with respect to the Fermi level. An important consequence is that supercurrent is influenced significantly by phase-coherent normal reflections, which may lead to an oscillation of supercurrent as a function of interlayer thickness or electronic momentum. These effects are strongest in low dimensions. Supercurrent oscillations as a function of a gate voltage in S-2DEG-S junctions were observed by Takayanagi et al. (1995a).

For most practical applications the universal features of supercurrent in a three-dimensional SINIS junction are of interest, where proper averaging over the resonances is important. The theory of stationary properties in such structures was developed by Brinkman and Golubov (2000), Galaktionov and Zaikin (2002), and Ozana et al. (2002). For thin interlayers, the existence of the coherent regime was demonstrated when supercurrent averaged over the transmission resonances was proportional to the barrier transparency $D$. With increasing thickness, the supercurrent became incoherent, of the order of $D^{2}$, as expected for two uncorrelated sequential tunneling processes. Quantitative calculations were carried out by Brinkman and Golubov (2000) and Galaktionov and Zaikin (2002) in order to study the crossover between these two regimes. The results are briefly summarized below.

In a clean SINIS junction, the Eilenberger equations are in general not applicable because of interference between the two interfaces. Starting from a set of Gor'kov equations, Brinkman and Golubov (2000) found an expression for the Green's functions in a clean threedimensional SINIS structure. Here $\mathrm{N}$ is either a normal metal or a weak superconductor $\left(\mathrm{S}^{\prime}\right)$ with $T_{c}^{\prime}<T_{c}$. In the case of symmetric low-transparency barriers the supercurrent is given by

$$
\begin{aligned}
I_{s}= & \frac{e}{\hbar} \int \frac{d^{2} k_{\|}}{(2 \pi)^{2}} T \sum_{\omega>0} \\
& \times \frac{\Delta_{1}^{2} \sin \varphi+\Delta_{1} \Delta_{2} \sqrt{E_{1} / E_{2}} d / \xi_{x} W^{2} \sin \frac{\varphi}{2}}{2 W^{4} E_{1}^{2}\left(\cosh d / \xi_{x}-\cos 2 k_{x} d\right)+E_{3}^{2}} .
\end{aligned}
$$

\footnotetext{
${ }^{8}$ These include those of Kresin (1986), Gudkov et al. (1988), Zaitsev (1990), Furusaki et al. (1991, 1992), Schüssler and Kümmel (1993), Chrestin et al. (1994), Golub and Horovitz (1994), Rittenhouse and Graybeal (1994), Volkov et al. (1995), Riedel et al. (1996), Tang, Wang, and Zhang (1996), Tang, Wang, and Zhu (1996), Wendin and Shumeiko (1996a, 1996b), Buttcher and Kopp (1997), Gogadze and Kosevich (1998), Johansson et al. (1999), Nevirkovets and Shafranjuk (1999), Samuelsson et al. (2000), Shchelkachev (2000), Kuhn et al. (2001), Lodder and Nazarov (2002), and Otadoy and Lodder (2002).
} 
Here $k_{\|}$is the wave-vector component parallel to the interfaces, $\quad E_{1}=\sqrt{\omega^{2}+\Delta_{1}^{2}}, \quad E_{2}=\sqrt{\omega^{2}+\Delta_{2}^{2}}, \quad E_{3}$ $=\sqrt{\omega^{2}+\Delta_{1}^{2} \cos ^{2} \varphi / 2}$, and $W=W_{1,2} / \hbar v_{x} \gg 1$, where $W_{1,2}$ is the strength of the interface potential $V(x)=W_{1} \delta(x)$ $+W_{2} \delta(x-d)$, and $\Delta_{1}, \Delta_{2}$ are the magnitudes of the pair potentials in the $\mathrm{S}$ and $\mathrm{N}$ layers, respectively. Further, $v_{x}=v_{F} \cos \theta, k_{x}=k_{F} \cos \theta$, and $\xi_{x}=\hbar v_{F} \cos \theta / 2 \omega$, where $v_{F}$ is the Fermi velocity and $k_{F}$ the Fermi momentum in the interlayer. For the case of a normal interlayer, $\Delta_{2}=0$, a more general expression for the supercurrent for arbitrary barrier transparency was obtained by Galaktionov and Zaikin (2002), which agrees with Eq. (99) in the relevant limits.

Equation (99) describes the interplay between the quasiparticle Breit-Wigner and Andreev resonances for arbitrary values of the parameter $k_{F} d$. It is instructive to consider this crossover in terms of the width of the transmission resonances in a double-barrier junction, which in the symmetrical case is given by $\Gamma$ $=\hbar v_{F}\langle x D(x)\rangle / 2 d=\hbar v_{F} / 8 d \widetilde{W}^{2}$, where $\langle x D(x)\rangle$ is the angle-averaged transparency of a single barrier $(x$ $=\cos \theta$ ). The transmission resonance occurs as a result of interference between normally reflected electrons inside the double-barrier structure, and the width $\Gamma$ can be interpreted as the inverse drag time of an electron inside the structure due to multiple normal reflections.

It was proven by Galaktionov and Zaikin (2002) that the supercurrent given by Eq. (99) for large $k_{F} d$ is equivalent to that obtained directly from the quasiclassical Eilenberger equations, while in the case of more than two interfaces (e.g., a SININIS junction) the quasiclassical approach does not work due to quantummechanical interference from multiple interfaces. For a detailed discussion of transport through multiple interfaces, see also Ozana et al. (2002).

Nonquasiclassical techniques, in particular, the dynamical mean-field theory, have recently been applied to theoretical descriptions of SINIS junctions [see the review article by Freericks et al. (2002) and references therein].

The supercurrent of a SINIS junction is controlled by a single parameter $\gamma_{\mathrm{eff}}$,

$$
\gamma_{\mathrm{eff}}=\frac{\pi T_{c}}{\Gamma}=\frac{2 \pi T_{c} d}{\hbar v_{F}\langle x D(x)\rangle},
$$

which is equal to the ratio of the widths of Andreev and Breit-Wigner resonances. Since the ratio $2 \pi T_{c} / \hbar v_{F}$ $\sim k_{e}-k_{h}$, where $k_{e, h}$ are the wave vectors of the electrons and holes forming the Andreev bound states, and since $d /\langle x D(x)\rangle$ is the average path $L$ of the particles inside the $\mathrm{N}$ layer, the parameter $\gamma_{\mathrm{eff}}$ determines the dephasing of the Andreev bound states. We shall call the regime of small $\gamma_{\mathrm{eff}}$, when there is no dephasing, the coherent regime.

\section{Coherent regime (broad resonances)}

As follows from Eq. (99), the coherent regime applies for a thin interlayer when the transmission resonances are broad, $\Gamma \gg \pi T_{c}$. The supercurrent is then given by

$$
I_{S}(\varphi)=\frac{e}{\hbar} \int \frac{d^{2} k_{\|}}{(2 \pi)^{2}} T \sum_{\omega} \frac{\Delta_{1}^{2} \sin \varphi}{E_{1}^{2} D_{2}^{-1}-\Delta_{1}^{2} \sin ^{2} \varphi / 2},
$$

where $D_{2}$ is the transparency of a double barrier NININ contact, which has a resonant structure, $D_{2}^{-1}$ $=1+\left(2 W \cos k_{x} d+2 W^{2} \sin k_{x} d\right)^{2}$. Integration over the directions of $k_{\|}$(over the resonances) yields the supercurrent

$$
e I_{S}(\varphi) R_{N}=2 \pi T \sum_{\omega>0} \frac{\Delta_{1}^{2} \sin \varphi}{\sqrt{\omega^{2}+\Delta_{1}^{2}} \sqrt{\omega^{2}+\Delta_{1}^{2} \cos ^{2} \varphi / 2}},
$$

which does not depend on the properties of the interlayer and coincides with the dirty-limit result (Kupriyanov and Lukichev, 1988a). Here $R_{N}^{-1}$ $=e^{2} k_{F}^{2} \gamma / 4 \pi^{2} \hbar$ is the normal-state contact resistance per square, where $\gamma=\langle x D(x)\rangle=1 / 4 W^{2}$. This expression can be generalized to the asymmetric case: $e I_{S}(\varphi) R_{N}=2 \pi T \Sigma_{\omega}\left|\Delta_{1}\right|^{2} \sin \varphi / E_{1} E_{3}^{\prime}, \quad$ with $E_{3}^{\prime}$ $=\sqrt{\omega^{2}+\left|\Delta_{1}\right|^{2}\left(\cos ^{2} \varphi / 2+\gamma_{-}^{2} \sin ^{2} \varphi / 2\right)}, \quad \gamma_{-}=\left(\gamma_{1}-\gamma_{2}\right) /\left(\gamma_{1}\right.$ $\left.+\gamma_{2}\right), \gamma_{1,2}=\left\langle x D_{1,2}(x)\right\rangle$, where $D_{1,2}$ are the individual barrier transparencies. In this case, $R_{N}^{-1}=e^{2} k_{F}^{2} \gamma_{c} / 2 \pi^{2} \hbar$, where $\gamma_{c}=\gamma_{1} \gamma_{2} /\left(\gamma_{1}+\gamma_{2}\right)$.

For $T=0$ the maximum value of $e I_{S}(\varphi) R_{N}$ is achieved at $\varphi \approx 1.86$ and exceeds the $e I_{C} R_{N}$ value of $(\pi / 2)\left|\Delta_{1}\right|$ for a tunnel SIS contact (see Fig. 7).

Equation (102) is identical to that found by Kupriyanov and Lukichev (1988a) for a SINIS junction with a diffusive interlayer in the same limit of $\gamma_{\mathrm{eff}} \ll 1$ [see Eq. (118) below]. This means that Eq. (118) is more general than the model used to derive it, since it holds even when the dirty-limit condition is violated in the interlayer.

Note that in the coherent regime of $\gamma_{\mathrm{eff}} \ll 1$ the supercurrent in a SINIS junction scales with $\langle x D(x)\rangle$ (similar to the SIS case) and not with $\langle x D(x)\rangle^{2}$. The reason is that the transmission resonances are broader than $\Delta$ for $\gamma_{\text {eff }} \ll 1$.

Brinkman and Golubov (2000) have shown that Eq. (102) can be derived by integrating the supercurrent per single channel over the transmission eigenvalue density for a normal double-barrier contact. This is proven by integrating $\int_{0}^{1} I_{C}(D) \rho(D) d D$ with $I_{C}(D)$ from Eq. (15) and with the density function $\rho(D)=\left(\hbar / e^{2} R_{N}\right) D^{-3 / 2}(1$ $-D)^{-1 / 2}$ for a double-barrier contact (Beenakker, 1997). Note that the same function $\rho(D)$ describes a disordered barrier [see Eq. (38)], which was applied by Naveh et al. (2000) to high- $J_{c}$ SIS junctions.

An analytical continuation of Eq. (102) provides the spectral supercurrent in the coherent regime (Brinkman and Golubov, 2000):

$$
\operatorname{Im} I_{E}=\operatorname{Im} \frac{\Delta_{1}^{2} \sin \varphi}{\sqrt{\Delta_{1}^{2}-E^{2}} \sqrt{\Delta_{1}^{2} \cos ^{2}(\varphi / 2)-E^{2}}} .
$$

It follows from Eq. (103) that supercurrent is carried by Andreev bound states in the energy range $\Delta_{1} \cos \varphi / 2<E<\Delta_{1}$. The reason for this broad energy 
distribution is that all channels with transmission coefficients $0 \leqslant D \leqslant 1$ contribute to the supercurrent.

\section{Incoherent regime (narrow resonances)}

In this case, $\Gamma \ll \pi T_{c}$ or, equivalently, $\gamma_{\mathrm{eff}} \gg 1$. With an increase in interlayer thickness, the coherent regime breaks down due to dephasing of the transmission resonances. After performing the angle averaging in Eq. (99) we obtain the general expression for a double-barrier junction

$$
\begin{aligned}
e I_{S}(\varphi) R_{N}= & 2 \pi T \sum_{\omega>0} \frac{\Delta_{1}^{2} \sin \varphi}{E_{1}^{2}} \int_{0}^{1} \frac{4 x^{5} d x}{\tilde{W}^{2} \sqrt{a^{2}-1}} \\
& +\frac{\Delta_{1} \Delta_{2} \sin \frac{\varphi}{2}}{E_{1} E_{2}} \frac{d}{\xi_{s^{\prime}}} \int_{0}^{1} \frac{8 x^{2} d x}{\sqrt{a^{2}-1}},
\end{aligned}
$$

where $a=\cosh \left(d / \xi_{x}\right)+\left(1 / 2 W^{4}\right) E_{3} / E_{1}$. The pair potential in $\mathrm{S}^{\prime}$ is determined self-consistently with

$$
-\Delta_{2} \ln \frac{T}{T_{c}^{\prime}}=2 \pi T \sum_{\omega>0}\left(\frac{\Delta_{2}}{\omega}-\left\langle F_{2}\right\rangle\right),
$$

where $\left\langle F_{2}\right\rangle$ is the angle-averaged anomalous Green's function in the interlayer,

$$
\left\langle F_{2}\right\rangle=4 \int_{0}^{1}\left(\frac{\Delta_{2}}{E_{2}} \sinh \left(\frac{d}{\xi_{x}}\right)+\frac{2 \Delta_{1}}{E_{1}} x^{4} \cos \frac{\varphi}{2}\right) \frac{x d x}{\sqrt{a^{2}-1}} .
$$

It follows from Eq. (104) that at low temperatures the CФR has two components, determined by first and second terms on the right-hand side of Eq. (104). The sign of the second term is determined by the sign of the interlayer pair potential $\Delta_{2}$, which is determined selfconsistently from Eq. (105) and depends on the sign of the effective electron-electron interaction in a weak superconducting $\left(\mathrm{S}^{\prime}\right)$ material. Due to the proximity effect, the interlayer pair potential $\Delta_{2}$ does not vanish at temperatures $T>T_{c}^{\prime}$. If the effective electron-electron interaction is attractive, $\Delta_{2}$ has a positive sign, while for a repulsive interaction $\Delta_{2}$ is negative at $T>T_{c}^{\prime}$ (where $T_{c}^{\prime}$ is defined using the absolute value of the coupling constant). Thus the measurements of a CФR in a SIS' IS junction can in principle be used for measuring the sign of the electron-electron interaction in metallic films (Brinkman and Golubov, 2000). A quantitative study of the corresponding corrections to the C $\Phi \mathrm{R}$ in various limits and, in particular, of the influence of nonequilibrium effects on these corrections has not yet been performed.

As follows from Eq. (104), for $T>T_{c}^{\prime}$ and $\gamma_{\mathrm{eff}} \gg 1$ the supercurrent is second order in $\langle x D(x)\rangle$,

$$
e I_{S}(\varphi) R_{N}=\frac{32 \pi T}{7 \gamma_{\text {eff }}} \sum_{\omega>0} \frac{\Delta_{1}^{2} \sin \varphi}{E_{1}^{2}} .
$$

Therefore, with increasing $\gamma_{\text {eff }}$, a crossover occurs from direct tunneling with a nonsinusoidal C $\Phi R$ and large $I_{C} R_{N}$ to sequential tunneling with sinusoidal CФR and $I_{C} R_{N} \propto 1 / \gamma_{\mathrm{eff}}$.

\section{B. SINIS junctions, dirty limit}

\section{The general case}

Now we consider the situation in which the interlayer is in the dirty limit $\ell \ll\left(d, \xi_{2}\right)$ with $\xi_{2}=\sqrt{D_{N} / 2 \pi T_{c}}$. In this case the stationary Josephson effect in a SINIS structure was analyzed in the framework of the Usadel equations by Kupriyanov and Lukichev (1988a), Zaitsev (1991), Golubov and Kupriyanov (1994), and Kupriyanov et al. (1999), using various approximations.

Kupriyanov et al. (1999) performed a detailed analysis in the practically interesting regime of small interlayer thickness $d \ll \xi_{2}$ and obtained the following general expression for the CФR:

$$
\begin{aligned}
\frac{I_{S}(\varphi) R_{N}}{2 \pi T_{c}}= & \frac{\pi T}{\gamma_{\mathrm{eff}}} \sum_{\omega>0} \frac{\Delta_{1}^{2} G^{2} \sin (\varphi)}{\omega^{2} \widetilde{\Omega}} \\
& +\frac{T}{T_{c}} \sum_{\omega>0} \frac{G \Delta_{1} \Delta_{2}}{\widetilde{\Omega} \omega} \frac{\sin (\varphi)}{\eta(\varphi)},
\end{aligned}
$$

where

$$
\begin{aligned}
& \gamma_{\mathrm{eff}}=\frac{\gamma_{B 1} \gamma_{B 2}}{\gamma_{B 2}+\gamma_{B 1}} d, \quad \gamma_{-}=\frac{\gamma_{B 1}-\gamma_{B 2}}{\gamma_{B 2}+\gamma_{B 1}}, \\
& q=g \frac{\pi T_{c}}{\gamma_{\mathrm{eff}} \omega}, \quad \eta(\varphi)=\sqrt{\cos ^{2}\left(\frac{\varphi}{2}\right)+\gamma_{-}^{2} \sin ^{2}\left(\frac{\varphi}{2}\right)},
\end{aligned}
$$

and

$$
\begin{aligned}
& G=\omega / \sqrt{\omega^{2}+\Delta_{1}^{2}}, \\
& \widetilde{\Omega}=\sqrt{\omega^{2}(1+q)^{2}+\left[q \Delta_{1} \eta(\varphi)+\Delta_{2}\right]^{2}} .
\end{aligned}
$$

The magnitude of the pair potential in the interlayer $\Delta_{2}$ is determined by the self-consistency equation,

$$
\Delta_{2}\left\{\ln \frac{T}{T_{c}^{\prime}}+2 \pi T \sum_{\omega>0}\left[\frac{1}{\omega}-\frac{1}{\widetilde{\Omega}}\right]\right\}=2 \pi T \sum_{\omega>0}\left[\frac{q \Delta_{1} \eta(\varphi)}{\widetilde{\Omega}}\right] .
$$

The first term in Eq. (108) describes the direct coupling of the superconducting electrodes, while the second term describes the contribution to the supercurrent due to sequential tunneling in two SIS' junctions connected in series. The interplay between these two channels depends on the barrier parameters $\gamma_{B 1,2}$, temperature, and the ratio between $T_{c}^{\prime}$ and $T_{c}$.

Figure 19 shows the results of a calculation of the critical current in a SINIS junction with a selfconsistently determined pair potential in both the clean and dirty limits. The coherent (I) and incoherent (II) regimes are indicated. III is the regime of two SIS' tunnel junctions connected in series for $T<T_{c}^{\prime}$. In the coherent regime I the supercurrent does not depend on whether the interlayer is in the clean or dirty limit. 


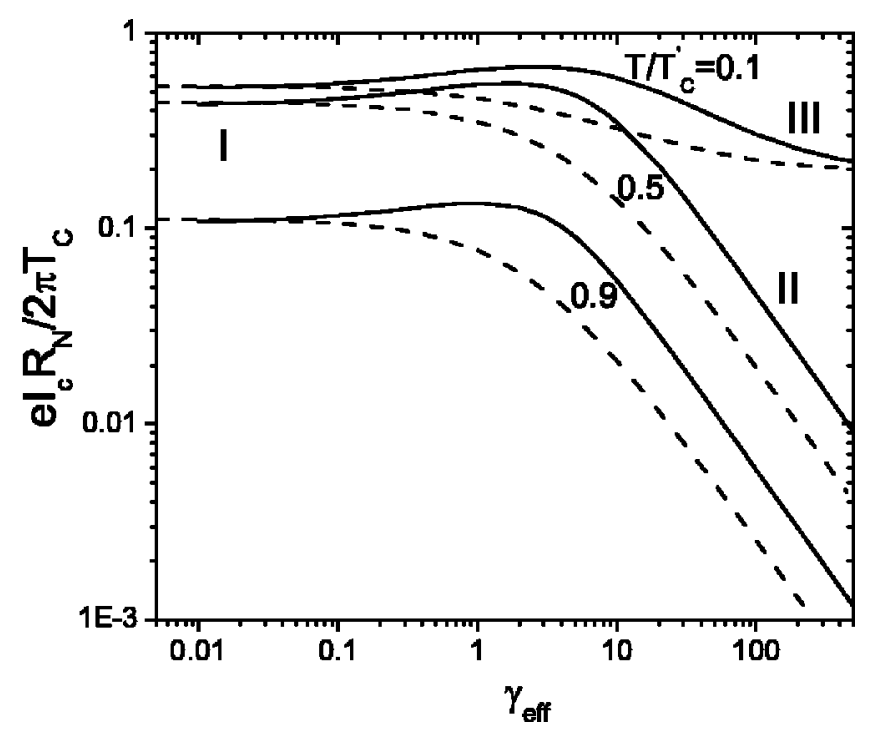

FIG. 19. $I_{C} R_{N}$ product in a SINIS junction in the clean limit (solid lines) and the dirty limit (dashed lines) for the ratio $T_{c} / T_{c}^{\prime}=7.4$ corresponding to the $\mathrm{Nb} / \mathrm{Al}$ combination. Adapted from Brinkman and Golubov, 2000.

Below we shall consider several limiting cases in which the CФR can be calculated analytically.

\section{Limit of high temperatures}

If the temperature is high enough to guarantee the inequality

$$
\pi T \gg \frac{\gamma_{\mathrm{eff}} \Delta_{2}}{1+\gamma_{\mathrm{eff}}}+\frac{\Delta_{1}}{1+\gamma_{\mathrm{eff}}},
$$

the magnitude of the interlayer order parameter $\Delta$ $\propto \eta(\varphi)$ and the CФR is sinusoidal. For $T$ close to $T_{c}$, the self-consistency equation $\Delta_{2}$ can be solved analytically in two limits of small and large values of $\gamma_{\text {eff }}$. For $\gamma_{\text {eff }} \ll 1$ the supercurrent is given by (Kupriyanov et al., 1999)

$$
I_{S}(\varphi) R_{N}=\frac{\Delta_{1}^{2}}{\pi T_{c}}\left\{\frac{\pi^{2}}{4}+\frac{\gamma_{\mathrm{eff}} \ln ^{2}\left(1 / 2 \gamma_{\mathrm{eff}}\right)}{\ln \left(T_{c} / 2 \gamma_{\mathrm{eff}} T_{c}^{\prime}\right)}\right\} \sin (\varphi),
$$

while for $\gamma_{\mathrm{eff}} \gg 1$

$I_{S}(\varphi) R_{N}=\frac{\Delta_{1}^{2} \sin (\varphi)}{4 \pi T_{c} \gamma_{\mathrm{eff}}}\left\{7 \zeta(3)+\frac{\gamma_{\mathrm{eff}} \pi^{4} / 4}{\pi^{2} / 4+\gamma_{\mathrm{eff}} \ln \left(T_{c} / T_{c}^{\prime}\right)}\right\}$.

For small $\gamma_{\text {eff }}$ Eq. (112) coincides with the AslamazovLarkin result, Eq. (10). For large $\gamma_{\text {eff }}$ it follows from Eq. (113) that the $I_{C} R_{N}$ product is enhanced by an increase of the $T_{c}^{\prime}$.

\section{Limit of low temperatures}

If $T \ll T_{c}^{\prime}$ and $\gamma_{\mathrm{eff}} \gg T_{c} / T_{c}^{\prime}$, the maximum value of the supercurrent flowing through the boundaries $I$ $\sim \sigma_{s^{\prime}} \Delta_{1} \Delta_{2} / \xi_{s^{\prime}} \gamma_{B}$ is much smaller than the depairing cur- rent in the interlayer material. In this regime the structure is equivalent to two junctions in series and the CФR is given by

$$
I_{S}(\varphi) R_{N}=2 \pi T \sum_{\omega>0} \frac{\Delta_{1} \Delta_{2}}{\sqrt{\left(\omega^{2}+\Delta_{1}^{2}\right)\left[\omega^{2}+\Delta_{2}^{2}(T)\right]}} \frac{\sin (\varphi)}{\eta(\varphi)} .
$$

The critical current is achieved at

$$
\cos (\varphi)=\min \left\{\frac{\gamma_{B 1}}{\gamma_{B 2}}, \frac{\gamma_{B 2}}{\gamma_{B 1}}\right\}
$$

and is equal to

$$
\begin{aligned}
I_{C} R_{N}= & 2 \pi T \sum_{\omega>0} \frac{\Delta_{1} \Delta_{2}}{\sqrt{\left(\omega^{2}+\Delta_{1}^{2}\right)\left[\omega^{2}+\Delta_{2}^{2}(T)\right]}} \\
& \times \frac{\gamma_{B 1}+\gamma_{B 2}}{\max \left\{\gamma_{B 1}, \gamma_{B 2}\right\}} .
\end{aligned}
$$

At $T=0$, Eq. (114) yields

$$
I_{S}(\varphi)=\frac{I_{C 1} I_{C 2} \sin (\varphi)}{\sqrt{I_{C 1}^{2}+I_{C 2}^{2}+2 I_{C 1} I_{C 2} \cos (\varphi)}},
$$

where $I_{C 1}$ and $I_{C 2}$ are the critical currents of each of two consecutive Josephson junctions. For a symmetric structure Eq. (117) yields the CФR $\sin (\varphi / 2)$.

\section{Limit of small $\gamma_{\text {eff }}$}

As follows from Eqs. (108) and (110), direct coupling is dominant for $\gamma_{\text {eff }} \ll 1$ and the CФR is determined by

$$
I_{S}(\varphi) R_{N}=2 \pi \sum_{\omega>0} \frac{\Delta_{1}^{2} \sin (\varphi)}{\sqrt{\omega^{2}+\Delta_{1}^{2}} \sqrt{\omega^{2}+\left[\Delta_{1} \eta(\varphi)\right]^{2}}} .
$$

As soon as $\eta(\varphi) \leqslant 1$ at any $\varphi$, the $I_{C} R_{N}$ product of a SINIS junction in this limit is larger than that for a symmetric SIS junction with a gap $\Delta_{1}$ in both electrodes. At $T=0$, Eq. (118) yields

$$
I_{S}(\varphi) R_{N}=\Delta(0) K\left(\sqrt{1-\gamma_{-}^{2}} \sin \frac{\varphi}{2}\right) \sin \frac{\varphi}{2},
$$

where $K(x)$ is the elliptic integral of the first kind. At $\gamma_{-}=0$, critical current is achieved at $\varphi=1.86$ with an $I_{C} R_{N}$ product $22 \%$ larger than in an SIS junction. In the symmetric case Eq. (118) coincides with Eq. (102) for a clean SINIS junction.

\section{Limit of large $\gamma_{\text {eff }}$}

In the limit of large $\gamma_{\text {eff }}$ there are three characteristic temperature intervals in the $I_{C}(T)$ dependence.

At temperatures larger than the critical temperature of the interlayer material

$$
T / T_{c}^{\prime} \gtrsim 1+\left[\sqrt{T_{c}\left(T_{c}-T_{c}^{\prime}\right)} /\left(\gamma_{\mathrm{eff}} T_{c}^{\prime}\right)\right]^{2 / 3},
$$




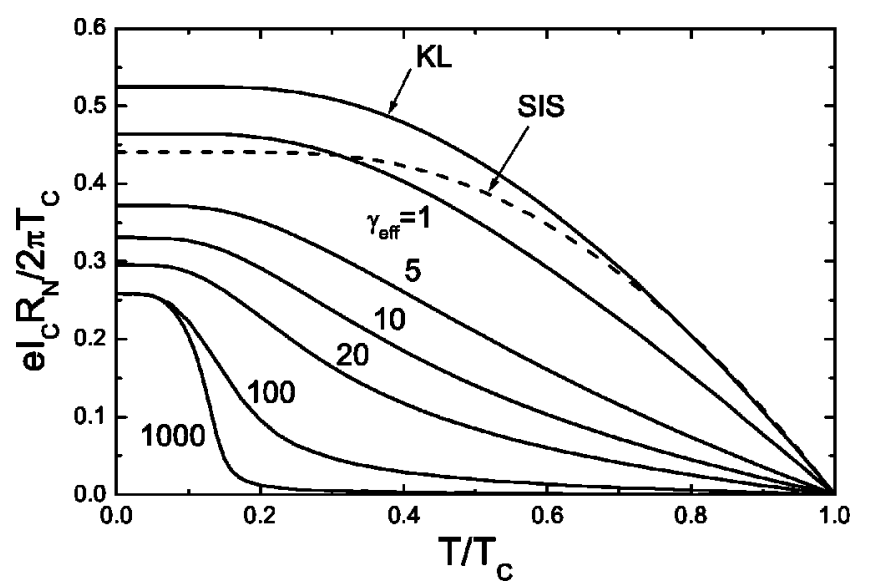

FIG. 20. Temperature dependence of critical current in a symmetric SINIS junction in the dirty limit: KL, KupriyanovLukichev curve, described by Eq. (102); SIS, junction that corresponds to the Ambegaokar-Baratoff curve. Adapted from Kupriyanov et al., 1999.

the CФR is sinusoidal. A general expression for the CФR was derived by Kupriyanov et al. (1999). The $I_{C} R_{N}$ product in this case is proportional to $\gamma_{\mathrm{eff}}^{-1}$.

In the rather narrow temperature interval

$$
\left|T / T_{c}^{\prime}-1\right| \lesssim T / T_{c}^{\prime} \lesssim 1+\left[\sqrt{T_{c}\left(T_{c}-T_{c}^{\prime}\right)} /\left(\gamma_{\mathrm{eff}} T_{c}^{\prime}\right)\right]^{2 / 3},
$$

$I_{S}(\varphi)$ is modified

$$
\frac{I_{S}(\varphi) R_{N}}{2 \pi T_{c}} \propto \frac{\sin (\varphi)}{[\eta(\varphi)]^{2 / 3}} .
$$

Finally, for $T \ll T_{c}^{\prime}$, the C $\Phi \mathrm{R}$ corresponds to two tunnel junctions in series and is given by Eq. (114).

Figure 20 summarizes the temperature dependencies of $I_{C} R_{N}$ in a symmetric SINIS junction in the dirty limit for various values of $\gamma_{\mathrm{eff}}$ and $T_{c}^{\prime}$. The sinusoidal CФR is realized at large $\gamma_{\text {eff }}$ and high temperatures. The CФR for small $\gamma_{\mathrm{eff}}$ is described by Eq. (118), for low temperatures by Eq. (114) and for close to $T_{c}^{\prime}$ by Eq. (119).

\section{SFS JOSEPHSON JUNCTIONS}

There is a continuously growing interest in charge and spin transport in contacts between superconductors and ferromagnets. For a long time, the observation of Josephson coupling in SFS junctions was a serious challenge. The solution to the problem of measuring supercurrents in SFS Josephson junctions was found by employing dilute ferromagnetic alloys. The first experimental observation of supercurrents in SFS junctions and the crossover from 0 to $\pi$ state in $\mathrm{Nb} / \mathrm{Cu}_{x} \mathrm{Ni}_{1-x} / \mathrm{Nb}$ Josephson junctions was reported by Ryazanov et al. (2000, 2001a, 2001b) and Veretennikov et al. (2000), and further phase-sensitive measurements were reported by Ryazanov et al. (2002). According to Eq. (8), the $\pi$ state is characterized by a phase shift of $\pi$ in the ground state of a junction and is formally described by the negative critical current $I_{C}$ in the $\operatorname{C\Phi R~} I_{S}(\varphi)=I_{C} \sin (\varphi)$.
The possibility of a $\pi$ state was first predicted by Bulaevskii et al. (1977) in a Josephson tunnel junction with magnetic impurities localized in the barrier. Bulaevskii et al. (1977) also predicted that a superconducting ring containing a $\pi$ junction could generate a spontaneous current and magnetic flux.

The discovery of a $\pi$ junction by Ryazanov et al. (2000, 2001a, 2001b) and Veretennikov et al. (2000) stimulated further experimental activity in this field and led to the observation of new phenomena in Josephson junctions with interlayers made from various ferromagnetic alloys (Kontos et al., 2001, 2002; Blum et al. 2002; Surgers et al., 2002).

At the same time, the theory of SFS $\pi$ junctions also attracted widespread interest. The physics of the 0-to- $\pi$ crossover in SFS junctions was studied theoretically from several different approaches. ${ }^{9}$ In addition to the study of $\pi$ transitions, new intriguing predictions have been made concerning a complex CФR.

Recently, the generation of a long-range triplet order parameter was predicted in structures with inhomogeneous magnetization or with noncollinear orientations of magnetization in different $\mathrm{F}$ layers (Bergeret et al., 2001c; Kadigrobov et al., 2001; Volkov et al., 2003). The Josephson effect in junctions between unconventional superconductors across different types of magnetic barriers was studied theoretically by Tanaka and Kashiwaya (1999, 2000).

The variety of systems exhibiting $\pi$ states include planar SFS proximity-effect structures, tunnel junctions with a magnetic insulator or magnetically active interfaces, and structures with barriers containing more than one magnetic layer. In this section, we shall concentrate on the basic features of the Josephson effect in junctions containing weak homogeneous metallic ferromagnets with collinear magnetization directions.

\section{A. Proximity effect in SF bilayer}

\section{The formalism}

Consider a bilayer consisting of a semi-infinite superconductor and a ferromagnet, separated by an interface with arbitrary transparency. Superconducting correlations induced in a ferromagnet differ qualitatively from those in SN proximity systems. Generally, the proximity effect can be understood as a penetration of a Cooperpair amplitude into a nonsuperconducting material. An electron and a hole having opposite spins and momenta are correlated via Andreev reflection (see Fig. 1), thus

\footnotetext{
${ }^{9}$ See, for example, Buzdin et al. (1982, 1992), Buzdin and Kupriyanov (1991), Radović et al. (1991), Tanaka and Kashiwaya (1997a), Procić et al. (1999), Dobrosavljević-Grujić et al. (2000), Fogelström (2000), Bergeret et al. (2001a, 2001b), Chtchelkatchev et al. (2001, 2002), Krivoruchko and Koshina (2001a, 2001b), Kulić and Kulić (2001), Radović et al. (2001), Barash and Bobkova (2002), Barash, Bobkova, and Kopp (2002), Buzdin and Baladie (2002), Golubov et al. (2002a, 2002b).
} 
providing an extension of superconductivity into a nonsuperconducting region. In the NS bilayer at a nonzero temperature these correlations decay exponentially with distance from the interface to the normal metal due to a dephasing between the wave functions of electrons and holes. In an SF bilayer the correlated electrons and holes, having opposite spin directions, are under the exchange field of a ferromagnet. This results in an energy shift between these quasiparticles and the creation of a nonzero momentum $Q$ of Cooper pairs (Demler et al., 1997).

As a result, the amplitude of superconducting correlations oscillates spatially in the ferromagnetic metal as $\cos Q x$. The sign change of this amplitude is equivalent to periodic $0-\pi$ phase jumps at certain points in the ferromagnet. Such an oscillating Cooper-pair amplitude is an analog of the so-called Larkin-Ovchinnikov-FuldeFerrel (LOFF) state in magnetic superconductors (Fulde and Ferrel, 1964; Larkin and Ovchinnikov, 1964).

The oscillations in the ferromagnet decay with distance from the SF interface. There is a quantitative difference between the decay lengths of clean and diffusive ferromagnets. At $T=0$, the decay length in the dirty limit exactly coincides with the oscillation period (Buzdin and Kupriyanov, 1991; Radović et al., 1991). In the clean limit the decay length is infinite at $T=0$ (Buzdin et al., 1982) and is limited only by elastic impurity scattering (Bergeret et al., 2002) or spin-orbit scattering (Demler et al., 1997) and typically exceeds the oscillation period. Therefore spatial oscillations are easier to observe in cleaner systems, but there is no qualitative difference between these two regimes. Keeping in mind that most of the practical thin-film junctions are in the dirty limit, we shall concentrate on this regime below.

We consider a SF bilayer in which the dirty-limit conditions are fulfilled in the S and $\mathrm{F}$ metals. The SF interface is not magnetically active and can be described by the spin-independent parameters $\gamma$ and $\gamma_{B}$,

$$
\gamma_{B}=R_{B} / \rho_{F} \xi_{F}, \quad \gamma=\rho_{S} \xi_{S} / \rho_{F} \xi_{F},
$$

where $R_{B}$ is the specific resistance of the SF interface, and $\rho_{S(F)}$ is the resistivity of the $\mathrm{S}(\mathrm{F})$ layer, while the coherence lengths are related to the diffusion constants $D_{S(F)}$ as $\xi_{S(F)}=\sqrt{D_{S(F)} / 2 \pi T_{c}}$. This approach is valid for relatively weak ferromagnetic materials-the case we are mostly interested in, when the exchange integral is smaller than $0.1 \mathrm{eV}$ and spin-dependent corrections to resistivity can be neglected.

The problem is described by the Usadel equations, Eq. (A11) (see the Appendix). In a ferromagnet, the Matsubara frequency $\omega$ is replaced by the complex frequency $\widetilde{\omega}=\omega+i H$, where $H$ is the exchange energy. Thus the following parametrization can be used in a ferromagnet:

$$
G_{\omega}=\frac{\widetilde{\omega}}{\sqrt{\widetilde{\omega}^{2}+\Phi_{\omega} \Phi_{-\omega}^{*}}}, \quad F_{\omega}=\frac{\Phi_{\omega}}{\sqrt{\widetilde{\omega}^{2}+\Phi_{\omega} \Phi_{-\omega}^{*}}} .
$$

The Usadel equation for the ferromagnet has the form

$$
\xi_{F}^{2} \frac{\pi T_{c}}{\widetilde{\omega} G_{F}} \frac{\partial}{\partial x}\left[G_{F}^{2} \frac{\partial}{\partial x} \Phi_{F}\right]-\Phi_{F}=0,
$$

while in the S layer $\widetilde{\omega}_{k}=\omega$. The boundary conditions at the SF interface $\left(x=d_{F}\right)$ are

$$
\begin{aligned}
& \frac{\xi_{S} G_{S}^{2}}{\omega} \frac{\partial}{\partial x} \Phi_{S}=\gamma \frac{\xi_{F} G_{F}^{2}}{\widetilde{\omega}} \frac{\partial}{\partial x} \Phi_{F}, \\
& \gamma_{B} \frac{\xi_{F} G_{F}}{\widetilde{\omega}} \frac{\partial}{\partial x} \Phi_{F}=G_{S}\left(\frac{\Phi_{S}}{\omega}-\frac{\Phi_{F}}{\widetilde{\omega}}\right) .
\end{aligned}
$$

One can directly see from Eq. (122) that the effective coherence length in a ferromagnet is complex. This point will be discussed in detail in the following subsection. Another interesting fact is that $\Phi_{S}$ near the SF interface is a complex function. This is a consequence of the nonlocal nature of the proximity effect: the influence of the exchange field on Cooper pairs penetrates into a superconductor at the distance $\xi_{S}$ (Buzdin and Kupriyanov, 1991; Krivoruchko and Koshina, 2002).

\section{Complex coherence length}

Far from the SF interface in a ferromagnet, the Usadel equations can be linearized $\left(G_{F}=1\right)$. Then it follows from Eq. (122) that the solution in a ferromagnet has the form

$$
\Phi(x) \propto e^{-x / \tilde{\xi}_{F}} e^{-x / \xi_{F 1}} e^{-i x / \xi_{F 2}},
$$

with the complex coherence length $\widetilde{\xi}_{F}$ given by

$$
\widetilde{\xi}_{F}=\sqrt{\frac{\hbar D_{F}}{2(\omega+i H)}} .
$$

The main consequence of the complex coherence length $\widetilde{\xi}_{F}$ is that $\Phi(x)$ oscillates with a period defined by $\xi_{F 2}$ and the oscillations decay on the scale of $\xi_{F 1}$. Further, in a strong ferromagnet, when $T \ll H$, one can neglect $\omega$ in the denominator of Eq. (126) and get $\xi_{F 1}=\xi_{F 2}$ $=\sqrt{\hbar D_{F} / H}$, i.e., in a strong diffusive ferromagnet both scales exactly coincide. On the other hand, for a weak ferromagnet the exchange field and the temperature are equally important, which yields (Ryazanov et al., 2001a, 2001b)

$$
\xi_{F 1,2}=\sqrt{\frac{\hbar D_{F}}{\left[H^{2}+(\pi T)^{2}\right]^{1 / 2} \pm \pi T}} .
$$

Here, the Matsubara frequency $\omega=\pi T$ for $n=0$ was taken for simplicity (an exact treatment will be presented below).

For $H \gg T$, Eq. (127) yields $\xi_{F 1}=\xi_{F 2}=\sqrt{\hbar D_{F} / H}$ as encountered with classical ferromagnets $(\mathrm{Fe}, \mathrm{Co}, \mathrm{Ni})$ where $H \sim 1 \mathrm{eV}$ is much higher than superconducting $T_{c}$.

On the other hand, in a weak ferromagnet $T \approx H$. In that case the decay length $\xi_{F 1}$ increases with decreasing temperature whereas $\xi_{F 2}$ decreases. As a result, a temperature variation changes the oscillation period and therefore is able to change the phase of the superconducting order parameter at a given point in the ferro- 
magnet from a 0 to a $\pi$ value (Heikkilä et al., 2000; Ryazanov et al., 2001a, 2001b). This fact, together with the large decay length $\xi_{F 1}$ for a small value of $H$ (long-range proximity effect), makes weak ferromagnets very interesting for experimental investigation.

Let us compare these results with those for a clean ferromagnet. In this case, as follows directly from the Eilenberger equations (Buzdin et al., 1982), the coherence length is also complex and $\widetilde{\xi}_{F}=\hbar v_{F} / 2(\omega+i H)$, where $v_{F}$ is the Fermi velocity in a ferromagnet. In a strong ferromagnet, $T \ll H, \quad \widetilde{\xi}_{F} \approx \hbar v_{F} / 2 i H$ is purely imaginary, which corresponds to an oscillating order parameter without decay. A detailed treatment (Bergeret et al., 2002) has revealed that the order parameter decays at the scale of the mean free path $l \gg \xi_{0}$, where $\xi_{0}$ $=\hbar v_{F} / 2 \pi T_{c}$ is the standard clean-limit coherence length.

\section{Phase variation at the SF interface}

A jump of the superconducting phase is possible at the SF interface if a relatively low transparent barrier is present (Krivoruchko and Koshina, 2001a, 2001b; Golubov et al., 2002a).

In the limit of small F-layer thickness,

$$
d_{F} \ll \min \left(\xi_{F}, \sqrt{\frac{D_{F}}{2 H}}\right)
$$

the gradients in Eq. (122) are small and in first approximation on $d_{F} / \xi_{F}$ the solution of Eq. (122) has the form

$$
\Phi_{F}=\frac{\widetilde{\omega} \Phi_{S} G_{S}}{\omega\left(G_{S}+\widetilde{\omega} \gamma_{B M} / \pi T_{c}\right)}, \quad \gamma_{B M}=\gamma_{B} \frac{d_{F}}{\xi_{F}} .
$$

Further, if rigid boundary conditions hold $\left[\gamma /\left(1+\gamma_{B M}\right)\right.$ $\ll 1]$, then $G_{S}=\omega / \sqrt{\omega^{2}+\Delta^{2}}$, and one can calculate the anomalous Green's function $F_{F, \omega}=\Phi_{F, \omega} /$ $\sqrt{\widetilde{\omega}^{2}+\Phi_{F \omega} \Phi_{F,-\omega}^{*}}$, which defines the superconducting order parameter. The function $F_{F}$ is complex and the phase shift $\chi$ between $F_{F}$ and the pair potential $\Delta$ in a superconductor equals

$$
\chi=\frac{1}{2} \arctan \frac{q}{p}+\frac{\pi}{4}(1-\operatorname{sgn} p) \operatorname{sgn} H,
$$

where

$$
\begin{aligned}
& p=1+\frac{\omega^{2}-H^{2}}{\left(\pi T_{c}\right)^{2}} \gamma_{B M}^{2}+2 \frac{\omega^{2} \gamma_{B M}}{\pi T_{c} \sqrt{\omega^{2}+\Delta^{2}}}, \\
& q=2 \gamma_{B M} \frac{H \omega}{\pi T_{c}}\left(\frac{\gamma_{B M}}{\pi T_{c}}+\frac{1}{\sqrt{\omega^{2}+\Delta^{2}}}\right) .
\end{aligned}
$$

At low $\omega$, the phase shift $\chi$ monotonically increases with $H$, achieving its maximum value

$$
\chi_{\max }=\frac{\pi}{2}
$$

at $H^{*} \sim \pi T_{c} / \gamma_{B M}$. The characteristic exchange field $H^{*}$ increases with increasing interface transparency or de- creasing thickness $d_{F}$. Thus the phase rotation occurs at the interface between a superconductor and a ferromagnet.

\section{B. Current-phase relation in SFS junctions: Simple geometry}

The most prominent feature of the CФR of an SFS junction is the possibility of realizing the $\pi$ state. Below we shall discuss several mechanisms of $0-\pi$ transitions and their quantitative criteria in terms of S, F material parameters and the SF interface resistance. Further, under certain conditions new types of nontrivial CФR's can be realized, having two energy minima for $\varphi=0$ and $\varphi$ $=\pi$. We shall discuss the corresponding physical mechanisms in terms of the spin splitting of Andreev bound states.

\section{1. $0-\pi$ transitions due to oscillating order parameter}

We start from the simplest model of an SFS junction with a metallic ferromagnet: a one-dimensional geometry and a resistivity of the ferromagnet high enough so that rigid boundary conditions are fulfilled at the SF interface. This model was studied by Buzdin et al. (1982), who predicted the $\pi$ states for the first time. Buzdin et al. (1982) assumed that clean-limit conditions were fulfilled in a ferromagnet. Later Buzdin and co-workers (Buzdin and Kupriyanov, 1991; Buzdin et al., 1992) considered the experimentally more relevant case in which both $\mathrm{S}$ and $\mathrm{F}$ metals were in the dirty limit. Strong ferromagnetism was assumed such that $H \gg \pi T_{c}$. At temperatures near $T_{c}$ the Ginzburg-Landau equations were solved for this structure, leading to a sinusoidal CФR with critical current (Buzdin and Kupriyanov, 1991; Buzdin et al., 1992)

$$
\begin{aligned}
& I_{C} R_{N}=\frac{\pi \Delta^{2}}{4 e T_{c}} y \frac{\sinh y \cos y+\cosh y \sin y}{\sinh ^{2} y \cos ^{2} y+\cosh ^{2} y \sin ^{2} y}, \\
& y=\frac{d_{F}}{\xi_{F}} \sqrt{\frac{H}{2 \pi T_{c}}} .
\end{aligned}
$$

Here, $d_{F}$ is the thickness of the $\mathrm{F}$ layer, $R_{N}$ is the resistance of the junction, and $\Delta$ is the value of the pair potential in a superconductor near the SF interface. Equation (134) describes damped oscillations of the critical current as a function of $d_{F}$, where the negative values of $I_{C}$ correspond to a $\pi$ junction. At large thickness the critical current decays as $I_{C} \propto \exp \left(-d_{F} / \xi_{F 1}\right)$, while the oscillation period is given by $2 \pi \xi_{F 2}$, with $\xi_{F 1,2}$ $=\sqrt{\hbar D_{F} / H}$ in accordance with Eq. (127). The critical current vanishes at $y \approx 3 \pi / 4+\pi n$.

At arbitrary $T$, analytical results can be obtained in the limit of large thicknesses, $y \gg 1$, and under rigid boundary conditions. The CФR is sinusoidal, with the critical current given by (Buzdin and Kupriyanov, 1991; Buzdin et al., 1992)

$$
I_{C} R_{N}=32 \sqrt{2} \frac{\Delta}{e} \mathcal{F}(\Delta / T) y \exp (-y) \sin (y+\pi / 4),
$$


where $\mathcal{F}(\Delta / T)$ is a monotonic function of temperature, $\mathcal{F}(\Delta / T)=(\pi / 128) \Delta / T_{c}$ at $T \approx T_{c}$, and $\mathcal{F}(\Delta / T) \approx 0.071$ at $T \ll T_{c}$. Near the transition temperature Eq. (135) coincides with Eq. (134). Thus the damped oscillatory behavior of $I_{C}$ vs $d_{F}$ holds over the whole temperature range.

Let us now consider the case of a weak ferromagnet, in which thermal energy $T$ is not negligible as compared to the exchange energy $H$ and in which the complex coherence length $\xi_{F 1,2}$ is given by Eq. (127).

If the interface transparency is low $\left(\gamma_{B} \gg 1\right)$, the Usadel equations in the $\mathrm{F}$ layer can be linearized and the supercurrent is given by (Ryazanov et al., 2001b)

$$
I_{S}=\frac{4 \pi T}{e R_{N}} \frac{d_{F}}{\gamma_{B} \xi_{F}} \operatorname{Re} \sum_{\omega>0} \frac{\Delta^{2} \sin (\varphi)}{\left(\omega^{2}+\Delta^{2}\right) \widetilde{d}_{F} \sinh \widetilde{d}_{F}}
$$

with $\widetilde{d}_{F}=d_{F} / \widetilde{\xi}_{F}$. Equation (136) is valid for sufficiently high temperatures, $T / T_{c} \gg d_{F} /\left(\xi_{F} \gamma_{B}\right)$. In this regime, the temperature dependence of $I_{C}$ is not sensitive to the value of $\gamma_{B}$, while the magnitude of the $I_{C} R_{N}$ product is suppressed as this parameter rises. It also follows from Eq. (136) that $I_{C}$ oscillates vs $d_{F}$ with the temperaturedependent period $\xi_{F 2}$ given by Eq. (127). This provides the possibility of $0-\pi$ crossover as temperature decreases, if $H \sim \pi T_{c}$ and $d_{F} \sim \xi_{F 2}$.

Such a crossover was observed by Ryazanov et al. (2001a, 2001b) in $\mathrm{Nb} / \mathrm{Cu}_{1-x} \mathrm{Ni}_{x} / \mathrm{Nb}$ Josephson junctions and explained using Eq. (136). Figure 21 shows the experimental data for $\mathrm{Nb} / \mathrm{Cu}_{1-x} \mathrm{Ni}_{x} / \mathrm{Nb}$ junctions for $x$ $=0.52$ (left panel). This crossover is reasonably well reproduced by the simple calculation using Eq. (136) (right panel).

At low $T$, Eq. (136) is not applicable, and nonlinear Usadel equations must be solved. The results of selfconsistent numerical calculations of the critical current in SFS junctions are presented in Fig. 22. Here, the more general case is considered when the F layer is split into two parts with parallel or antiparallel magnetization directions. $I_{C}$ decreases monotonically with $H$ for antiparallel magnetizations of the F layers, and the $0-\pi$ crossover takes place for the parallel case. Thus switching of the supercurrent between 0 and $\pi$ states may be achieved by changing the magnetization directions of the layers. A thermally induced $0-\pi$ crossover is shown in the inset of Fig. 22 using parameters that are relevant to the junctions studied by Ryazanov et al. (2001a, 2001b).

Therefore the theory based on the Usadel equations accounts reasonably well for the observed thermally induced crossover to the $\pi$ state in SFS junctions. Phasesensitive proof of the $\pi$ shift below the crossover temperature in $\mathrm{Nb} / \mathrm{Cu}_{1-x} \mathrm{Ni}_{x} / \mathrm{Nb}$ junctions was reported by Ryazanov et al. (2002).

\section{2. $0-\pi$ transitions due to phase jumps at the SF interfaces}

As was shown above, the phase of the order parameter $\chi$ is discontinuous at the SF interface if a potential barrier exists between the two metals see [Eq. (130)].

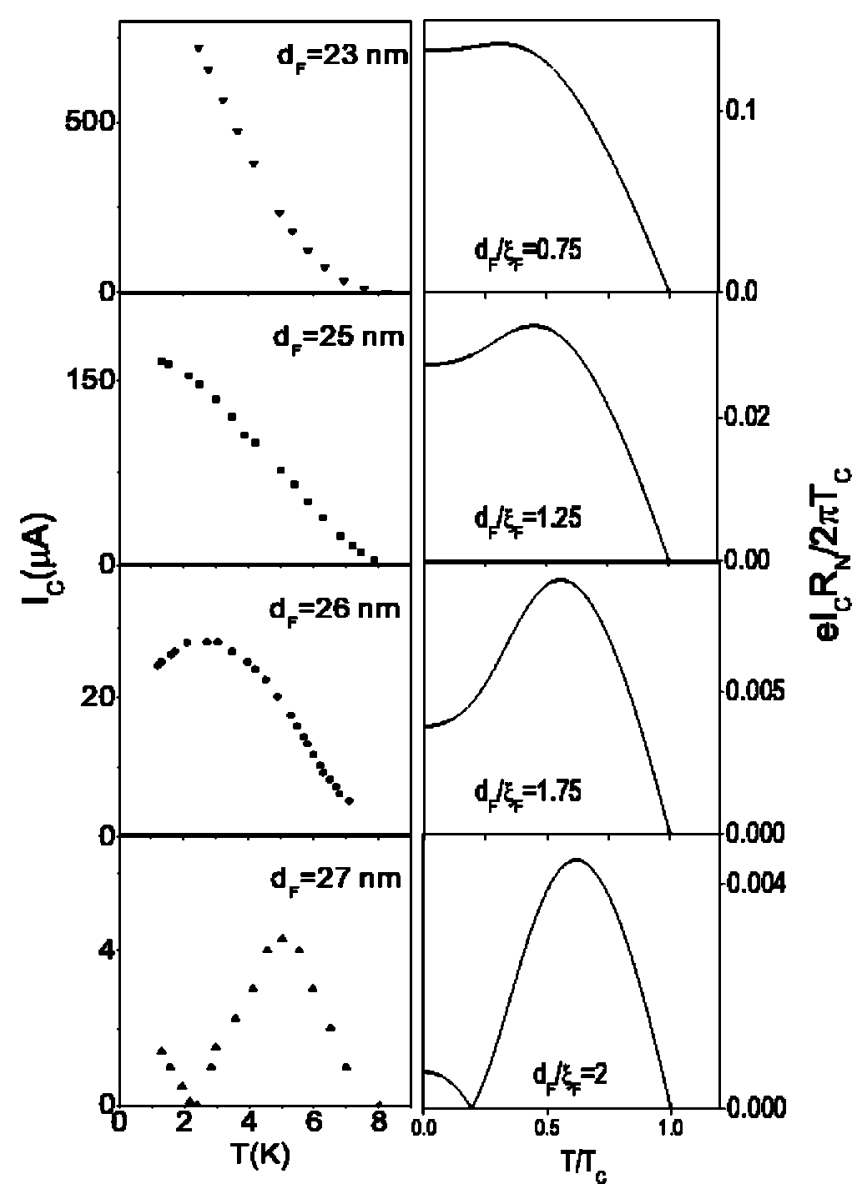

FIG. 21. Temperature dependence of critical current $I_{C}$ : left panel, $I_{C}$ as a function of temperature for $\mathrm{Nb}-\mathrm{Cu}_{0.48} \mathrm{Ni}_{0.52}-\mathrm{Nb}$ junctions with different F-layer thicknesses between 23 and 27 $\mathrm{nm}$ as indicated: right panel, model calculations of the temperature dependence of $I_{C}$ in a SFS junction from Eq. (136) for exchange energy $H=0.8 \pi T_{c}, \gamma_{B}=10$, and different values of the ratio $d_{F} / \xi_{F}$. Adapted from Ryazanov et al., 2001a.

Such phase jumps may lead to a $\pi$ transition even if the $F$-layer thickness is smaller than $\xi_{F 2}$ so that the order parameter in the ferromagnet does not oscillate (Krivoruchko and Koshina, 2001a, 2001b; Golubov et al., 2002a).

To illustrate this, consider a tunnel junction formed by two SF bilayers (SFIFS structure) with thin diffusive $\mathrm{F}$ layers, $d_{F} \ll \xi_{F}$. We also assume rigid boundary conditions to hold at both the SF interfaces. In this case, the general expression for the supercurrent has the form

$$
I=\frac{\pi T}{e R_{N}} \sum_{\omega} \operatorname{Im}\left[F_{F, L}^{*}\left(-H_{L}\right) F_{F, R}\left(H_{R}\right)\right],
$$

where $F_{F, L(R)}$ are solutions of the Usadel equation in the left (right) ferromagnetic layers [(Eq. 129)] and $R_{N}$ is the barrier resistance.

Let us consider for simplicity a symmetric structure in which the SF bilayers may differ only by the magnetization directions in the $\mathrm{F}$ layers and $\left|H_{L}\right|=\left|H_{R}\right|$. In this case, the CФR is sinusoidal, $I_{S}(\varphi)=I_{C} \sin (\varphi)$, with the critical current given by

$$
I_{C}=\frac{\pi T}{e R_{B, I}} \sum_{\omega} \frac{\left|\Phi_{S}\right|^{2}}{\omega^{2}+\left|\Phi_{S}\right|^{2}} \frac{\cos \Psi}{\left(p^{2}+q^{2}\right)^{1 / 2}},
$$




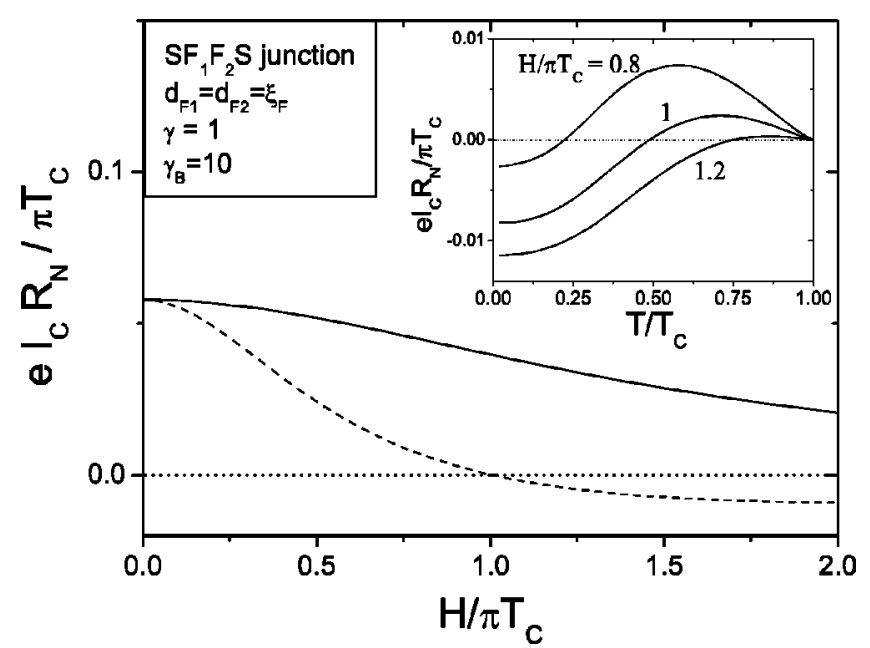

FIG. 22. Critical current in a SFS junction calculated selfconsistently at $T / T_{c}=0.5$ : solid line, antiparallel orientation of magnetizations; dashed lines, parallel magnetization; inset, thermally induced $0-\pi$ crossover in the parallel case. Adapted from Golubov et al., 2002a.

with $\Psi=\frac{1}{2}\left[\chi_{R}\left(H_{R}\right)-\chi_{L}\left(-H_{L}\right)\right]$, where the constants $p, q$ are given by Eqs. (131), (132), and the phases $\chi_{L, R}$ by Eq. (130).

For antiparallel orientation of the magnetizations, $H_{R}=-H_{L}$, the phase shift across the junction is $\Psi_{a}$ $=0$, since the phase drops at the two SF interfaces are subtracted from each other. For parallel orientation, $H_{R}=H_{L}$, we have

$$
\Psi_{p}=\arctan \frac{q}{p}+\frac{\pi}{2}(1-\operatorname{sgn} p) .
$$

In this case, the phase shifts at the two SF interfaces are added and provide the possibility of a $\pi$ state. Such a crossover happens for sufficiently large exchange fields. For $\left|H_{L, R}\right|<\pi T_{c} / \gamma_{B M}$, the parameter $p$ is positive, so that $\Psi_{p}<\pi / 2$ and the junction is always in the 0 state $\left[I_{S}(\varphi)=I_{C} \sin (\varphi)\right]$. However, with an increase of exchange field above the threshold value $\pi T_{c} / \gamma_{B M}$, the parameter $p$ changes sign at small $\omega$. As a result, a sharp transition to the $\pi$ state occurs at $H=\pi T_{c} / \gamma_{B M}$ and low $T$.

We emphasize that the $\mathrm{F}$ layers are thin, so that there are no spatial oscillations of the order parameter in these layers. There is no phase variation in the $\mathrm{S}$ layers either. Therefore the considered scenario of a 0- $\pi$ transition layer is totally related to the phase jumps at the SF interfaces. Indeed, according to Eq. (130), the phase drop $\chi$ between the Usadel functions $F_{S} \propto \Delta$ and $F_{F}$ $\propto \Delta \exp \{i \chi\}$ at each SF interface is equal to $\pi / 2$ in the limit of large $H$, providing the total $\pi$ shift between $F_{F, L}\left(-H_{L}\right)$ and $F_{F, R}\left(H_{R}\right)$ [it is the phase difference between these two functions that determines the supercurrent according to Eq. (137)].

At $H=\pi T_{c} / \gamma_{B M}$ and low temperatures, the critical current is enhanced logarithmically, $I_{c}^{(a)} \propto \ln \left(T_{c} / T\right)$ for antiparallel directions of the magnetizations. Such an enhancement of $I_{C}$ was demonstrated by Bergeret et al.

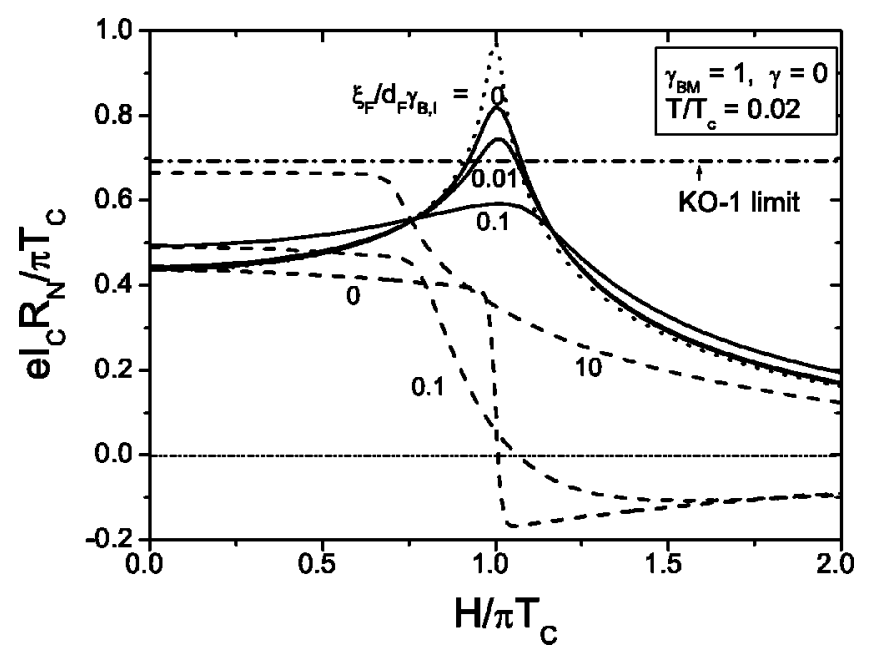

FIG. 23. Influence of temperature and barrier transparency on enhancement of the critical current (antiparallel magnetizations, solid lines) and the $0-\pi$ transition at which $I_{c}$ changes its sign (parallel magnetizations, dashed lines) in a SFIFS junction: dotted line, $T / T_{c}=0.01$ and $\xi_{F} / d_{F} \gamma_{B, I}=0$. Adapted from Golubov et al., 2002a.

(2001b) in a simple model. More detailed calculations were performed by Krivoruchko and Koshina (2001a, 2000b), Barash et al. (2002b), Chtchelkatchev et al. (2002), and Golubov et al. (2002a). It was shown that the origin of this effect is the exchange splitting of the proximity-induced density of states and the Andreev bound states in the $\mathrm{F}$ layers, which becomes equal to $\Delta$ at $H=\pi T_{c} / \gamma_{B M}$.

The above-mentioned results are illustrated in Fig. 23, where the critical current of an SFIFS junction is shown for parallel and antiparallel configurations. For arbitrary F-layer thicknesses and interface parameters, the boundary problem (121)-(122) can be solved by using a fully self-consistent numerical procedure (Golubov et al., 2002a), which includes self-consistency with the superfluid velocity $v_{s}$, essential (contrary to the constriction case) in the quasi-one-dimensional geometry.

Figure 23 demonstrates a sharp $0-\pi$ transition in a SFIFS junction in the parallel case at low temperature and a small-barrier transparency (described by the parameter $\gamma_{B, I} \gg 1$, which characterizes the barrier $I$ ). The transition broadens with decreasing $\gamma_{B, I}$ and shifts to large values of $H$. The same holds true for the magnitude of the $I_{C}$ enhancement in the antiparallel case: the peak magnitude drops with a decrease of the barrier strength, when the ratio $d_{F} \gamma_{B, I} / \xi_{F}$ becomes comparable to $T / T_{c}$.

Figure 24 illustrates the supercurrent flow in a SFIFS tunnel junction with two antiparallel-oriented ferromagnetic layers. Electrons and holes shift their energies upon crossing the barrier. Due to the spin flip under Andreev reflection, the reflected hole gains exactly the same energy as is lost by the electron. The corresponding resonance transmission leads to an $I_{C}$ enhancement in the antiparallel case (Chtchelkatchev et al., 2002; Golubov et al., 2002a). 


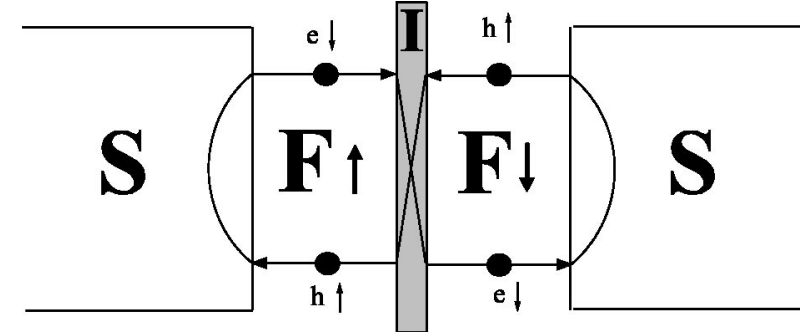

FIG. 24. Processes leading to critical current enhancement in SFIFS junction at antiparallel orientatons of the magnetizations at $H=\pi T_{c} / \gamma_{B M}$.

\section{Asymmetric case: SIFS tunnel junction}

A practically interesting geometry is the onedimensional SIFS tunnel junction, in which the superconducting phase drops at the tunnel barrier between the $\mathrm{S}$ and $\mathrm{F}$ materials. The CФR is sinusoidal in this case, but supercurrent oscillates as a function of the F-layer thickness $d_{F}$. Such a junction was realized recently by Kontos et al. (2002), who reported an oscillating dependence of $I_{C}$ on $d_{F}$.

The critical current is given by

$$
I_{C}=\frac{2 \pi T}{e R_{N}} \sum_{\omega>0} \frac{\Delta^{2}}{\omega^{2}+\Delta^{2}} \operatorname{Re}\left\{\frac{1}{\tilde{\gamma}_{B} \sinh \widetilde{d}_{F}+G_{S} \cosh \widetilde{d}_{F}}\right\},
$$

where $\tilde{\gamma}_{B}=\gamma_{B} \sqrt{\widetilde{\omega} / \pi T_{c}}, \widetilde{d}_{F}=d_{F} \sqrt{\widetilde{\omega} / \pi T_{c}}$. This expression is valid for arbitrary thickness $d_{F}$ and exchange-field $H$ assuming rigid boundary conditions at the FS interface. For large $d_{F}$ this expression describes damped oscillation behavior of $I_{C}$ vs $d$.

In the case of a strong ferromagnet, $H \gg \pi T_{c}$, a simple analytical expression for the critical current can be obtained. In the vicinity of the transition temperature and for highly transparent SF interfaces Buzdin and Baladie (2002) have obtained the critical current

$$
I_{C} R_{B, I}=\frac{\pi \Delta^{2}}{2 T_{c}} \frac{\cos y \cosh y}{\cos 2 y+\cosh 2 y}
$$

where $y=\left(d_{F} / \xi_{F}\right) \sqrt{H / 2 \pi T_{c}}$.

For arbitrary $T$ and low-transparency SF interfaces, when

$$
\gamma_{B} \sqrt{\frac{H}{2 \pi T_{c}}} \gg \operatorname{coth}\left(\frac{d_{F}}{\xi_{F}} \sqrt{\frac{H}{2 \pi T_{c}}}\right),
$$

one arrives at the expression

$$
I_{C} R_{B, I}=\frac{\pi \Delta}{4 e} \frac{(\sinh y \cos y-\cosh y \sin y)}{z\left(\sinh ^{2} y+\sin ^{2} y\right)} \tanh \left(\frac{\Delta}{2 T}\right),
$$

where $z=\left(\gamma_{B} / \sqrt{H / 2 \pi T_{c}}\right)$. A similar expression was used at low $T$ by Kontos et al. (2002) to fit their experimental data on $I_{C}\left(d_{F}\right)$ in SIFS junctions, and an excellent agreement with theory was reported.

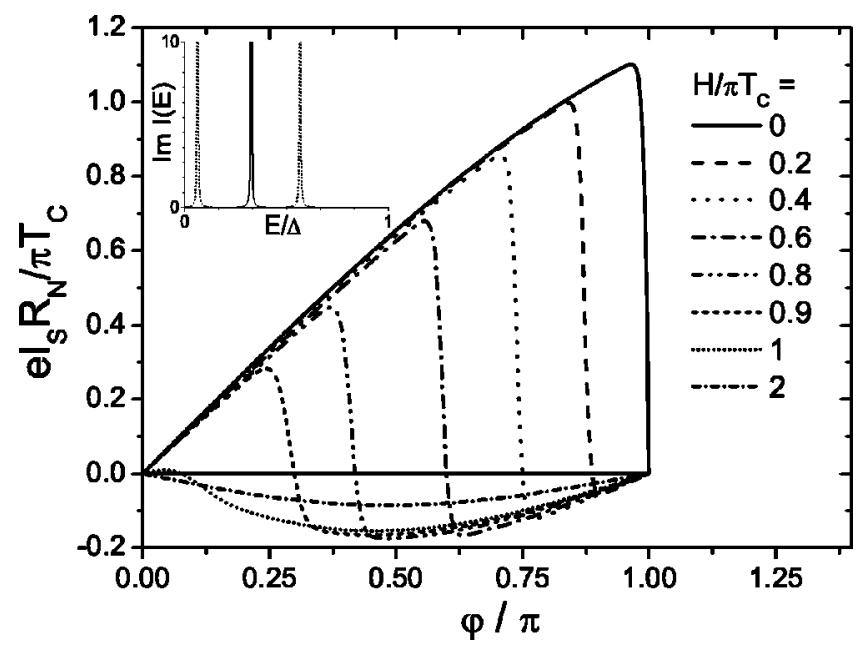

FIG. 25. Current-phase relation in a clean SFcFS junction with ideally transparent constriction $(D=1)$ at $T / T_{c}=0.01, \gamma_{B M}$ $=1$ for different values of the normalized exchange field $H$. Inset, spectral supercurrent density at $\varphi=2 \pi / 3$ for $H=0$ (solid line) and $H=0.4$ (dashed lines). Adapted from Golubov et al., $2002 b$.

\section{Clean SFcFS point contact}

A clean point contact, or constriction, connecting two superconducting SF bilayers (SFcFS) may exhibit rather peculiar $I_{S}(\varphi)$ dependences (Golubov et al., 2002b).

Assume that the constriction has a transparency $D$ and its size is much smaller than the mean free path, $a$ $\ll l$. For illustration, we consider a generic system in which both the $\mathrm{F}$ layers are thin, $d_{F} \ll \xi_{F}$, and their magnetization directions are parallel.

The supercurrent can be calculated by the general expression (19) with functions $f_{1,2}$ and $g_{1,2}$ in the $F$ layers. Since $f_{1,2}(-\omega) \neq f_{1,2}(\omega)$, the summation over $\omega$ should be extended from $-\infty$ to $\infty$.

In the symmetric junction, $I_{S}(\varphi)$ is determined by the expression

$$
\begin{aligned}
& I_{S}(\varphi)=\frac{4 \pi T}{e R_{N}} \sum_{\omega>0} \operatorname{Re} \frac{\Delta^{2} \sin \varphi}{W^{2}+\Delta^{2}\left[1-D \sin ^{2}(\varphi / 2)\right]}, \\
& W=\omega+\gamma_{B M} \widetilde{\omega} \sqrt{\omega^{2}+\Delta^{2}} / \pi T_{c} .
\end{aligned}
$$

At small $\omega$, the function $I_{S}(\varphi)$ changes its sign at the finite phase difference

$$
\varphi_{c}=2 \arcsin \sqrt{\left[1-\left(\gamma_{B M} H / \pi T_{c}\right)^{2}\right] / D}
$$

if the exchange field is in the range $1-D$ $<\left(\gamma_{B M} H / \pi T_{c}\right)^{2}<1$. The resulting CФR is shown in Fig. 25 .

In order to understand this result, we note that Eq. (142) has a form similar to the expression for supercurrent in a clean $\mathrm{ScS}$ point contact, with renormalized frequency $\omega \rightarrow W$. Going to the spectral current $\operatorname{Im} I(\varepsilon)$ by analytical continuation $\omega \rightarrow-i(\varepsilon+i 0)$, we see that $\operatorname{Im} J(E) \propto \Sigma_{i} \delta\left(E-E_{B i}\right)$. The Andreev bound-state energies $E_{B i}$ are the roots of the equation 


$$
E_{B}+\gamma_{B M}\left(E_{B}-H\right) \frac{\sqrt{\Delta^{2}-E_{B}^{2}}}{\pi T_{c}}= \pm \Delta \sqrt{1-D \sin ^{2} \frac{\varphi}{2}}
$$

For $\gamma_{B M}=0$ the standard result $E_{B}$ $= \pm \Delta \sqrt{1-D \sin ^{2}(\varphi / 2)}$ for an ScS contact is recovered, while in the presence of an exchange field this bound state splits into two (see inset in Fig. 25). At $\varphi=\varphi_{c}$ one of these split (positive) peaks crosses zero, leaving the domain $E>0$, and simultaneously a negative peak moves from the region $E<0$ into $E>0$, thus reversing the sign of the supercurrent.

The splitting of Andreev bound states in SFS junctions was first predicted by Kuplevakhskii and Fal'ko $(1990,1993)$ for an SFS junction either with a clean metallic ferromagnet with fully transparent interfaces or with a paramagnetic tunnel barrier. The splitting of the Andreev bound states was later demonstrated in various types of weak links involving ferromagnets by Tanaka and Kashiwaya (1997a), Dobrosavljević-Grujić et al. (2000), Fogelström (2000), Chtchelkatchev et al. (2001), Radović et al. (2001), Barash and Bobkova (2002), Golubov et al. (2002b).

The sign reversal of the supercurrent in SFS junctions can also be achieved at fixed $\mathrm{H}$ due to the nonequilibrium population of levels as discussed by Heikkilä et al. (2000) and Yip (2000).

For an antiferromagnetic orientation in the $F$ layers a 0 - $\pi$ transition does not occur due to a compensation of the phase shifts in both electrodes.

\section{E. Diffusive SFcFS point contact}

Let us now consider the case of a diffusive point contact connecting two SF bilayers. In a symmetric structure with rigid boundary conditions at the SF interfaces and thin $\mathrm{F}$ layers supercurrent can be derived from the Usadel equations (Golubov et al., 2002a, 2002b),

$$
\begin{aligned}
I_{S}(\varphi)= & \frac{4 \pi T}{e R_{N}} \operatorname{Re} \sum_{\omega>0} \frac{\Delta \cos (\varphi / 2)}{\sqrt{W^{2}+\Delta^{2} \cos ^{2}(\varphi / 2)}} \\
& \times \arctan \left(\frac{\Delta \sin (\varphi / 2)}{\sqrt{W^{2}+\Delta^{2} \cos ^{2}(\varphi / 2)}}\right),
\end{aligned}
$$

where $W$ is given by Eq. (143).

At $\gamma_{B M}=0$, Eq. (145) reproduces the KO-1 formula for diffusive $\mathrm{ScS}$ constrictions, in which case the supercurrent does not depend on the properties of the interlayer. Thus the exchange field does not influence $I_{S}(\varphi)$ if the SF interfaces are transparent, $\gamma_{B M}=0$. Still, for finite $\gamma_{B M}$, a stronger modification of the CФR takes place in an SFcFS contact than in an ScS contact. The calculation of $I_{S}(\varphi)$ using the Eq. (145) yields results similar to those for a clean point contact, with a less sharp transition from the 0 to the $\pi$ state (see Fig. 26).

The temperature dependence of the critical current in this case shows a thermally induced $0-\pi$ crossover with nonzero critical current at the transition point, in agree-

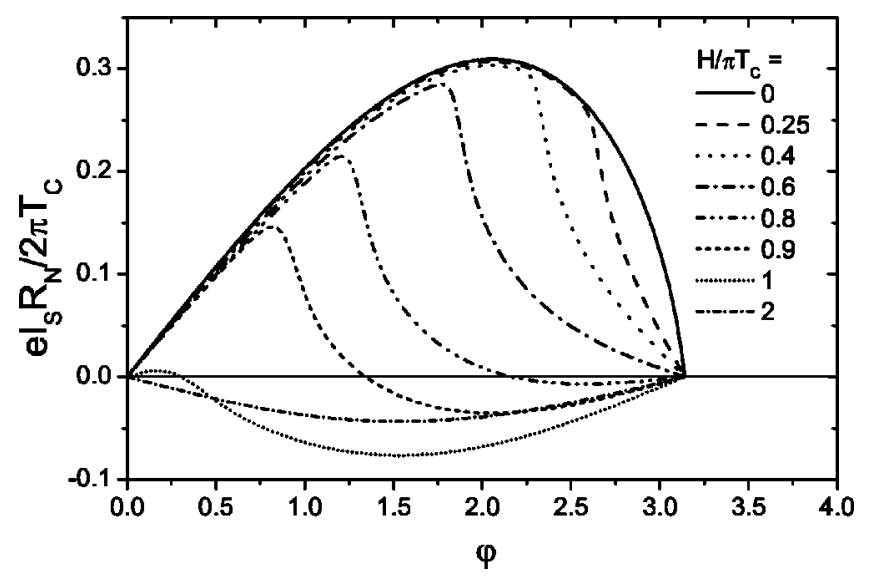

FIG. 26. The CФR in a diffusive SFcFS point contact at $T / T_{c}=0.01, \gamma_{B M}=1$ for different values of the exchange field $h$. Adapted from Golubov et al., 2002b.

ment with the results of Chtchelkatchev et al. (2001). The $I_{C}(T)$ curves are presented in Fig. 27. The nonvanishing supercurrent at the $0-\pi$ transition point is generally realized when higher harmonics are present in $I_{S}(\varphi)$ (a similar situation takes place in $d$-wave junctions; see Sec. X). The zero value of $I_{C}$ at the $0-\pi$ transition by Ryazanov et al. (2001a, 2001b; see Fig. 21) is due to the fact that the low-transparency regime (and hence a sinusoidal C(TR) was realized in that experiment.

Note that Eq. (145) can also be derived by an integration over the transmission distribution $\int_{0}^{1} \rho(D) I(D) d D$, where $I(D)$ is given by Eq. (142) and $\rho(D)$ is Dorokhov's distribution function, Eq. (18).

A C $\Phi \mathrm{R}$ of the type shown in Figs. 26 and 27 corresponds to the coexisting stable and metastable 0 and $\pi$ states. The consequences for a SQUID with ferromagnetic junctions were discussed theoretically by Radovic et al. (2001), who predicted new modes of the magneticflux penetration in such SQUID's. It was shown that the coexistence of integer and half-integer fluxoid configurations appears in both rf and dc SQUID's and generates two flux jumps per one external flux quantum.

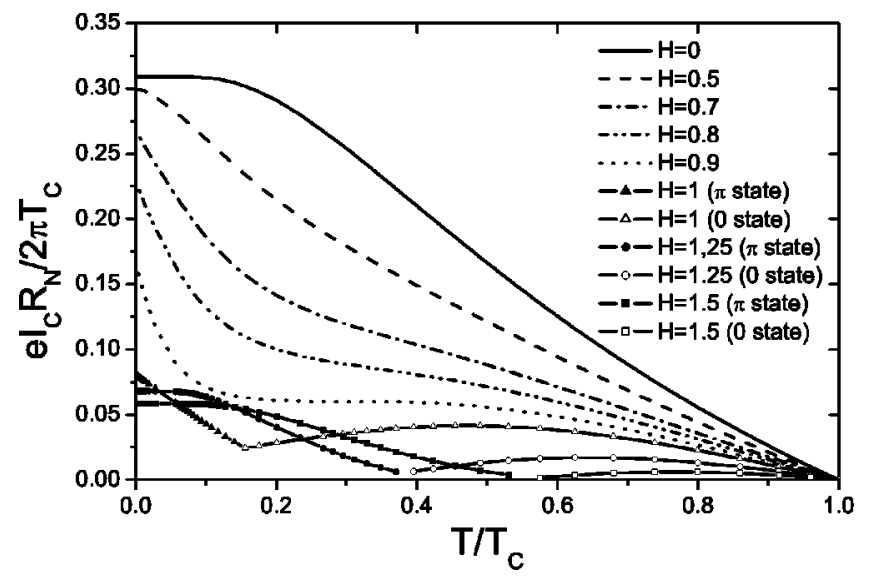

FIG. 27. Temperature dependence of the critical current in a diffusive SFcFS point contact at $\gamma_{B M}=1$ for different values of the exchange field $H$. 


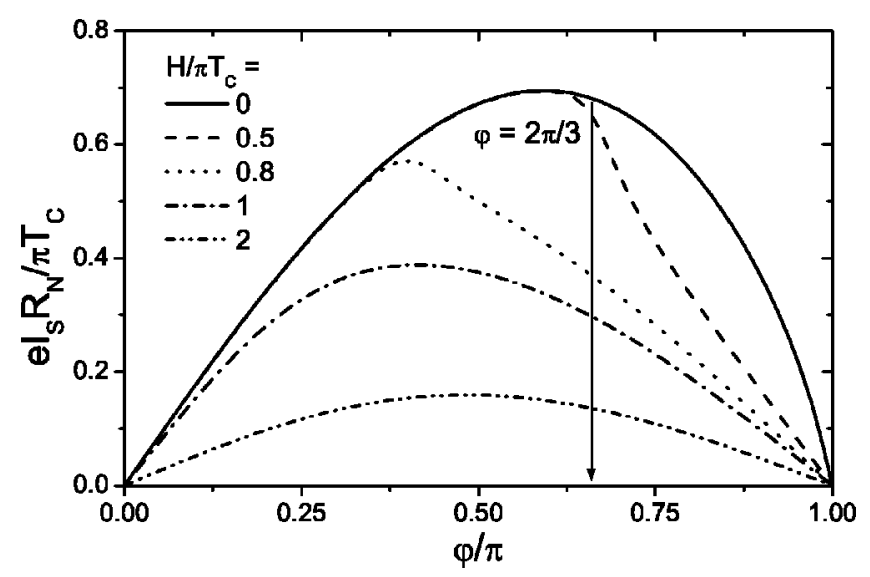

FIG. 28. The CФR in a double-barrier SIFIS junction at $T / T_{c}=0.02, \gamma_{B M}=1$ for different values of the exchange field $H$. Adapted from Golubov et al., 2002b.

\section{F. Double-barrier SIFIS junction}

A double-barrier SIFIS junction with a diffusive $\mathrm{F}$ interlayer is easier to implement experimentally than a SFcFS junction. Therefore we consider this case separately.

We assume that the condition $\gamma \ll \max \left(1, \gamma_{B}\right)$ is satisfied. In that case, rigid boundary conditions can be used. For low-transparency barriers, $\gamma_{B} \gg d_{F} / \xi_{F}$, the expression for the supercurrent is given by (Golubov et al., 2002b)

$$
I_{S}(\varphi)=\frac{2 \pi T}{e R_{N}} \operatorname{Re} \sum_{\omega>0} \frac{\Delta^{2} \sin \varphi}{\sqrt{\omega^{2}+\Delta^{2}} \sqrt{W^{2}+\Delta^{2} \cos ^{2}(\varphi / 2)}},
$$

where $W$ is given by Eq. (143). $I_{S}(\varphi)$ is strongly modified by the finite $H$ (see Fig. 28), especially at low temperatures. An increase in $H$ results in a shift of the $I_{S}(\varphi)$ maximum from $\varphi_{\max } \approx 1.86$ at $H=0$ to values smaller than $\pi / 2$. In the limit of large exchange fields, $H / \pi T_{c} \gg \gamma_{B M}^{-1}, I_{S}(\varphi)$ returns to the sinusoidal form.

The physical origin of these results can be clarified in a real-energy representation. Making an analytical continuation in Eq. (147), we obtain the spectral supercurrent [compare with Eq. (103) for a SINIS junction]

$$
\begin{aligned}
& \operatorname{Im} I_{E}=\operatorname{Im} \frac{\Delta_{0}^{2} \sin \varphi}{\sqrt{\Delta^{2}-E^{2}} \sqrt{\Delta^{2} \cos ^{2}(\varphi / 2)-\widetilde{E}^{2}}}, \\
& \widetilde{E}=E+\gamma_{B M}(E-H) \sqrt{\Delta^{2}-E^{2}} / \pi T_{c} .
\end{aligned}
$$

Equation (148) implies that at $\varphi_{c}=2 \arccos \left(\gamma_{B M} H / \pi T_{c}\right)$ singularities in $\operatorname{Im} I(E)$ are shifted to the Fermi level. At $\varphi>\varphi_{c}$ the negative singularity in $\operatorname{Im} I(E)$ for one spin projection crosses the Fermi level and appears in the positive energy domain, whereas the positive peak for the other projection leaves the domain $E>0$ (this process is illustrated in Fig. 29). As a result, the supercurrent starts to decrease at $\varphi>\varphi_{c}$ (see Fig. 28).

Figure 30 shows the temperature dependencies of the $I_{C} R_{N}$ product for various $H$ and $\gamma_{B M}=1$. For $H=0$, the

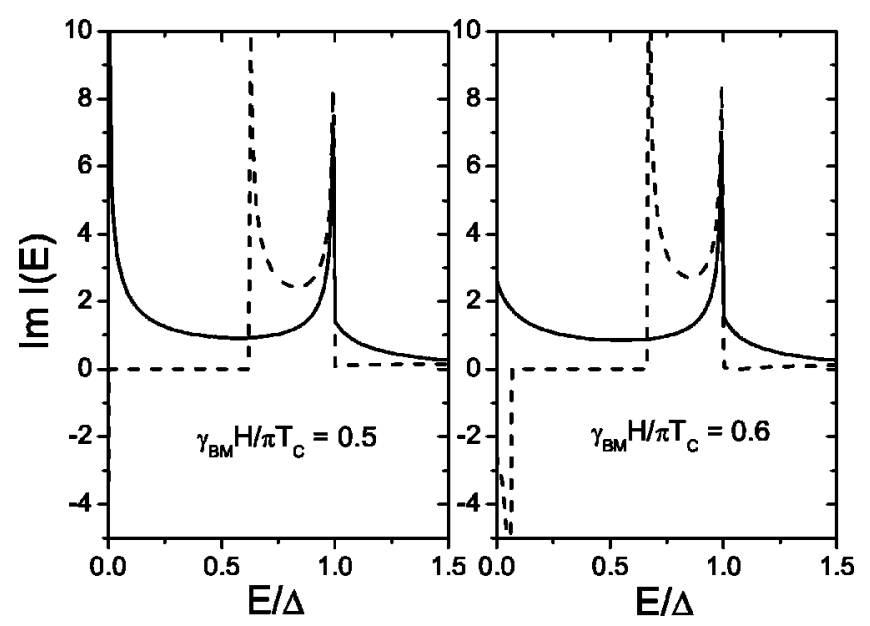

FIG. 29. Spectral supercurrent in a diffusive double-barrier junction with a thin ferromagnetic interlayer at $\gamma_{B M}=1, \varphi$ $=2 \pi / 3$ for two different values of the exchange field $H$. The chosen value of $\varphi$ corresponds to $\varphi_{c}$ at $\gamma_{B M} H / \pi T_{c}=0.5$. Adapted from Golubov et al., 2002 b.

Kupriyanov and Lukichev curve is reproduced with a maximum in $I_{S}(\varphi)$ at $\varphi_{m} \approx 1.86$. For larger $H$, thermal effects suppress $I_{C}$ at low $T$ due to the presence of low energy peaks of $\operatorname{Im} I(E)$. The strongest modifications of $I_{C}(T)$ take place for $H / \pi T_{c}=1$. In contrast to the SFIFS case considered above, there is no transition to the $\pi$ state in a double-barrier SIFIS structure. As was shown recently by Buzdin (2003), such a transition is possible in the case of very low-transparent SF interfaces.

The Josephson effect in ballistic double-barrier SIFIS planar junctions was studied theoretically by Radovic et al. (2003), who showed that in the case when the F metal is in the clean limit, coherent geometrical oscillations of the maximum Josephson current are superimposed on oscillations related to the crossovers between 0 and $\pi$ states.

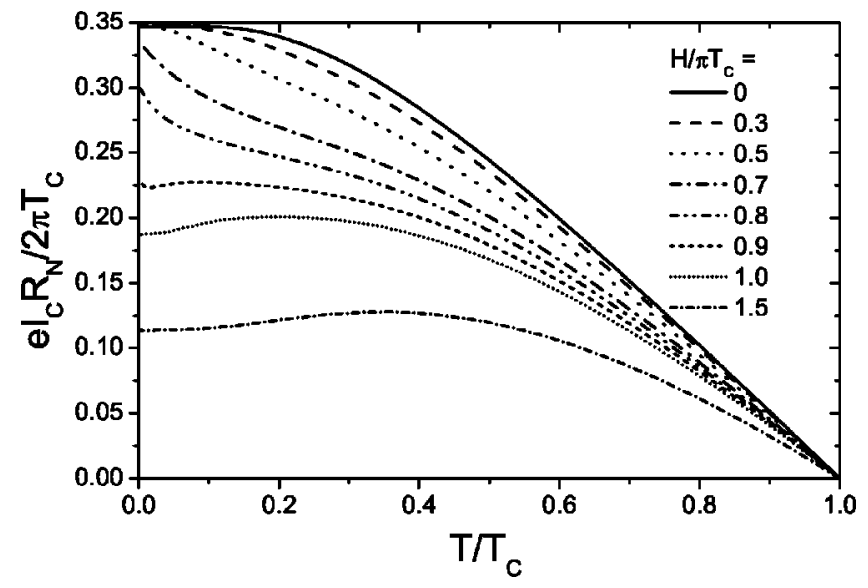

FIG. 30. The nonmonotonic temperature dependence of the critical current in a diffusive double-barrier junction with a thin ferromagnetic interlayer at $\gamma_{B M}=1$ and various values of the exchange field $H$. 


\section{EXPERIMENTAL METHOD OF MEASURING $I_{S}(\varphi)$ AND ITS APPLICATION}

From the above-mentioned consideration we can see that a study of the CФR may provide important information about a junction's parameters. Below, we shall describe the method for CФR measurement and some of its practical applications.

\section{A. Description of the experimental method}

Most commonly for the experimental investigation of the CФR, the weak link of interest is incorporated in a superconducting ring with a sufficiently small inductance $L$. This circuit is usually called a single-junction interferometer. When an external flux $\Phi_{e}$ is applied to a superconducting ring, circulating current is induced and the flux $\Phi_{i}$ through the ring is given by

$$
\Phi_{i}=\Phi_{e}-I L .
$$

Here $I$ is the full current in the loop. In this case the phase difference $\varphi$ across the weak link is

$$
\varphi=2 \pi \frac{\Phi_{i}}{\Phi_{0}}+2 \pi n
$$

where $\Phi_{0}$ is the flux quantum and $n$ is an integer number. In general, the total current may consist of several components. We consider below the situations in which the superconducting part $I_{S}(\varphi)=I_{C} f(\varphi)$ of the current essentially exceeds all other components, namely,

$$
\omega L / R_{N} \equiv \omega \tau \ll 1, \quad L C \omega^{2} \ll 1,
$$

where $R_{N}$ is the normal junction resistance and $C$ is the capacitance. Under this limitation we get from Eqs. (149) and (150)

$$
\varphi=\varphi_{e}-\beta f(\varphi)
$$

where $\varphi_{e}=2 \pi \Phi_{e} / \Phi_{0}$ is the normalized external flux, and the normalized critical current $\beta$ is defined as

$$
\beta=2 \pi L I_{c} / \Phi_{0} \text {. }
$$

Jackel et al. (1974, 1976) and Waldram and Lumley (1975) demonstrated that in this case the CФR can be obtained by monitoring $\varphi$ with a flux detector as a function of $\varphi_{e}$. In practice it is difficult to determine the flux $\Phi_{i}$ with the required sensitivity.

A more elegant method based on radio frequency, the rf technique, was proposed by Silver and Zimmerman (1967). The method was further developed by Rifkin and Deaver (1976), who showed that the CФR and the phase-dependent conductance could be extracted from experimental data without any fitting parameters.

In the $\mathrm{rf}$ method the interferometer and the weak link of interest are coupled through a mutual inductance $M$,

$$
M=k \sqrt{L L_{T}},
$$

to a tank circuit with known inductance $L_{T}$, capacitance $C_{T}$, and quality factor $Q$. Here $k$ is the coupling coefficient between the tank circuit and the interferometer. The tank circuit is driven by a dc bias current $I_{d c}$ and an rf current $I_{r f}$ of a frequency $\omega$ close to the resonant frequency $\omega_{0}=1 / \sqrt{L_{T} C_{T}}$ of the tank circuit. Usually in the experiment $Q \gg 1$ and $k \ll 1$ are chosen and the rf current in the tank circuit is sinusoidal. The total magnetic flux applied to the interferometer is $\Phi_{e}=\Phi_{d c}+\Phi_{r f}$. If the amplitude of $\Phi_{r f}$ is small, $\Phi_{r f} \ll \Phi_{0}$, then $\varphi_{e} \cong \varphi_{d c}$ and the C $\Phi \mathrm{R}$ can be determined by monitoring the effective impedance of the tank circuit-interferometer combination as a function of the externally applied magnetic flux.

Rifkin and Deaver (1976) and Il'ichev and co-workers (Il'ichev, Hoenig, et al., 2001; Il'ichev, Zakosarenko, Fritzsch, et al., 2001) have shown that if in addition to the restrictions given by Eq. (151) the condition of a small signal limit $\varphi_{r f} \ll 1+\beta f^{\prime}(\varphi)$ is valid, then the back influence of the superconducting loop on the tank circuit can be expressed via the effective tank circuit inductance $L_{\text {eff }}$ and the resistance $R_{\text {eff }}$,

$$
\begin{aligned}
& L_{\mathrm{eff}}=L_{T}\left(1-\frac{k^{2} \beta f^{\prime}(\varphi)}{1+\beta f^{\prime}(\varphi)}\right), \\
& R_{\mathrm{eff}}=R_{T}\left(1+\frac{k^{2} Q \omega \tau}{\left[1+\beta f^{\prime}(\varphi)\right]^{2}} \frac{\omega}{\omega_{0}}\right) .
\end{aligned}
$$

Here, $R_{T}$ determines the damping of the rf oscillations when the interferometer is decoupled. The phase angle $\alpha$ between the drive current $I_{r f}$ and the tank voltage $U$ is given by

$$
\tan \alpha=\frac{1}{R_{\text {eff }}\left(\varphi_{e}\right)} \cdot\left(\frac{1}{\omega C_{T}}-\omega L_{\text {eff }}\left(\varphi_{e}\right)\right) .
$$

It follows from Eqs. (155), (156), and (157) that at $\omega$ $=\omega_{0}$ and $\omega_{0} \tau \ll 1$ the phase angle is

$$
\tan \alpha=\frac{k^{2} Q \beta f^{\prime}(\varphi)}{1+\beta f^{\prime}(\varphi)}
$$

By combining

$$
d \varphi=\frac{d \varphi_{d c}}{1+\beta f^{\prime}(\varphi)}
$$

and Eq. (158) one can easily obtain

$$
I_{S}\left(\varphi_{d c}\right)=\frac{I_{c}}{k^{2} Q \beta} \int_{0}^{\varphi_{d c}} \tan \alpha\left(\varphi_{d c}\right) d \varphi_{d c} .
$$

The numerical factor in Eq. (160) can be obtained from experiment. Indeed, the mutual inductance $M$ is extracted from the period $\Delta I_{d c}$ of the $\alpha-I_{d c}$ dependence according to $M \Delta I_{d c}=\Phi_{0}$. Combining Eqs. (153) and (154) this factor can be rewritten as

$$
\frac{I_{c}}{k^{2} Q \beta}=\frac{L_{T}\left(\Delta I_{d c}\right)^{2}}{2 \pi Q \Phi_{0}} \text {. }
$$

The inductance $L_{T}$ can be measured in a conventional setup. The quality factor $Q$ can be determined from the resonant response curve of the tank circuit. Thus all the parameters in Eq. (160) are known. Note that for an analysis of experimental data no fitting parameter is needed.

If the parameter $\beta f^{\prime}(\varphi)$ in Eq. (159) is small, then the 
difference between $\varphi$ and $\varphi_{d c}$ is also negligible, $\varphi \simeq \varphi_{e}$, and Eq. (157) is simplified to

$$
\tan \alpha=k^{2} Q \beta f^{\prime}(\varphi), \quad \beta f^{\prime}(\varphi) \ll 1 .
$$

Moreover, since in experiments the typical value of $k^{2} Q \lesssim 1$, then $\tan \alpha \simeq \alpha$ and the measured dependence $\alpha\left(\varphi_{d c}\right)$ is proportional to the derivative of the C $\Phi \mathrm{R}$ $f^{\prime}(\varphi)$ with respect to the phase difference across the junction of interest.

\section{B. The range of validity of the method}

Let us now briefly discuss the physical limitations on the key parameters. First of all it is important to note that, in order to reconstruct the C $\Phi \mathrm{R}$ over the complete phase range $-\pi<\varphi<\pi$, the inequality

$$
\left|\beta f^{\prime}(\varphi)\right|<1
$$

should be fulfilled. Otherwise the C $\Phi \mathrm{R}$ becomes multivalued in the vicinity of $\varphi_{e}=\pi+2 \pi n$ [see Eq. (152)] and certain values of $\varphi$ cannot be fixed by applying an external magnetic flux.

For a given critical current of the Josephson element of interest, an upper limit for the loop inductance $L$ is set by the inequality (163). For moderate critical currents of the order of $20 \mu \mathrm{A}$, the inductance $L$ is $\simeq 10 \mathrm{pH}$, which is close to the practical geometrical limitations on $L$.

On the other hand, if the critical current is much smaller, say of the order of $10 \mathrm{nA}$, inequality (163) does not limit the loop inductance. Instead the fluctuation inductance $L_{f}$ restricts the value of $L$ [see Eq. (165) below]. That is why measurements of very small supercurrents, which become accessible by the method described here, are typically realized within the limit $\beta \ll 1$.

Il'ichev and co-workers (Il'ichev, Hoenig, et al., 2001; Il'ichev, Zakosarenko, Fritzsch, et al., 2001) have also shown that the condition $\beta \ll 1$ can be achieved by reducing the critical current even to the limit when the Josephson coupling energy $E_{J}=I_{C} \Phi_{0} / 2 \pi$ becomes smaller than the thermal energy $k_{B} T$. According to Khlus and Kulik (1975), an exponential reduction of the observable critical current with temperature occurs in this limit,

$$
I_{C} \rightarrow I_{C} \exp \left(-L / 2 L_{f}\right),
$$

where

$$
L_{f}=\left(\Phi_{0} / 2 \pi\right)^{2} / k_{B} T
$$

is the so-called fluctuation-threshold inductance. At $T$ $=4.2 \mathrm{~K}$ the value of $L_{f} \approx 1.7 \mathrm{nH}$ exceeds the geometrical inductance limit $(10 \mathrm{pH})$ by several orders of magnitude. Therefore the required interferometer inductance is $L \ll L_{f}$, permitting us to neglect the reduction of $I_{C}$, which can be easily realized experimentally.

If $L \sim L_{f}$, thermal noise modifies the internal flux in the ring and therefore the measured (apparent) C $\Phi R$ is also modified compared to the real CФR (see Jackel, et al., 1974; de Bruyn Ouboter and Omelyanchouk, 1995; Il'ichev, Zakosarenko, IJsselstein, et al., 1998a; Il'ichev, Zakosarenko, Schultze, et al., 2000). For instance, in a junction with a sinusoidal C $\Phi R$, the apparent CФR is deformed in such way that its maximum shift to a phase $\varphi_{\max }<\pi / 2$, and the slope of its curve at $\varphi=0$ become larger than unity. Nevertheless, Il'ichev, Zakosarenko, Schultze, et al. (2000) have shown that the real CФR can be reconstructed from the data, and thus the method is still applicable even for the case $L \sim L_{f}$.

Therefore, if the inequality (163) is fulfilled, then quite generally the CФR can be extracted from the data over the complete phase range by using Eqs. (152) and (160) without any fitting parameters. This method, being differential with respect to $\varphi$, provides a high sensitivity of the CФR measurement. In practice, however, this procedure works perfectly well when $\beta f^{\prime}(\varphi)$ is not close to unity. Indeed, even small thermal fluctuations and the applied rf flux $\varphi_{r f}$ can distort the $\varphi\left(\varphi_{e}\right)$ dependence and hence the apparent CФR, because for $\beta f^{\prime}(\varphi)=1$ the derivation $d \varphi / d \varphi_{e}$ diverges [see Eq. (159)].

\section{Measuring technique and calibration}

Several parameters of the measurement setup have been tested and applied for study of the current-phase relation. The inductance of the tank circuits was in the range of $10^{-2}-1 \mu \mathrm{H}$, providing resonant frequencies of 5-40 MHz. The unloaded quality factor measured for all tank circuits at different temperatures was in the range of $10^{2}<Q<10^{3}$. Usually the inductive coupling between an interferometer and a tank circuit was provided by a flip-chip configuration. In some particular cases the interferometer of interest was integrated with the coil on the same chip.

The interferometer inductances were in the range of $10-100 \mathrm{pH}$, providing $L \ll L_{f}$ for liquid-helium temperatures, where the deviation of the CФR from conventional sinusoidal form is expected. In earlier studies the tank voltage was detected by a room-temperature amplifier with high input impedance. In order to increase the sensitivity of the setup, Oukhanski et al. (2003) recently developed a cold amplifier with low noise temperature, which operates down to $300 \mathrm{mK}$. To satisfy the small signal limit, the measurements were carried out with an amplitude of $I_{r f}$, providing a flux of $\left(10^{-3}-10^{-1}\right) \Phi_{0}$ in the interferometer loop.

At present, several setups for C $\Phi \mathrm{R}$ measurements are available which work in the temperature range from $T_{c}$ down to $10 \mathrm{mK}$. External magnetic field is shielded by a magnetic shield or by a combination of magnetic and superconducting shields.

From the periodicity of $I_{S}(\varphi)$ it follows that $\int_{0}^{\pi} \tan \alpha\left(\varphi_{d c}\right) d \varphi_{d c}=0$. This condition permits subtraction of a constant phase shift coming from electronics and cables. Since the only sample characteristic entering Eq. (160) is $I_{S}(\varphi)$, the measurement system can be calibrated using samples with known critical current and 
(a)
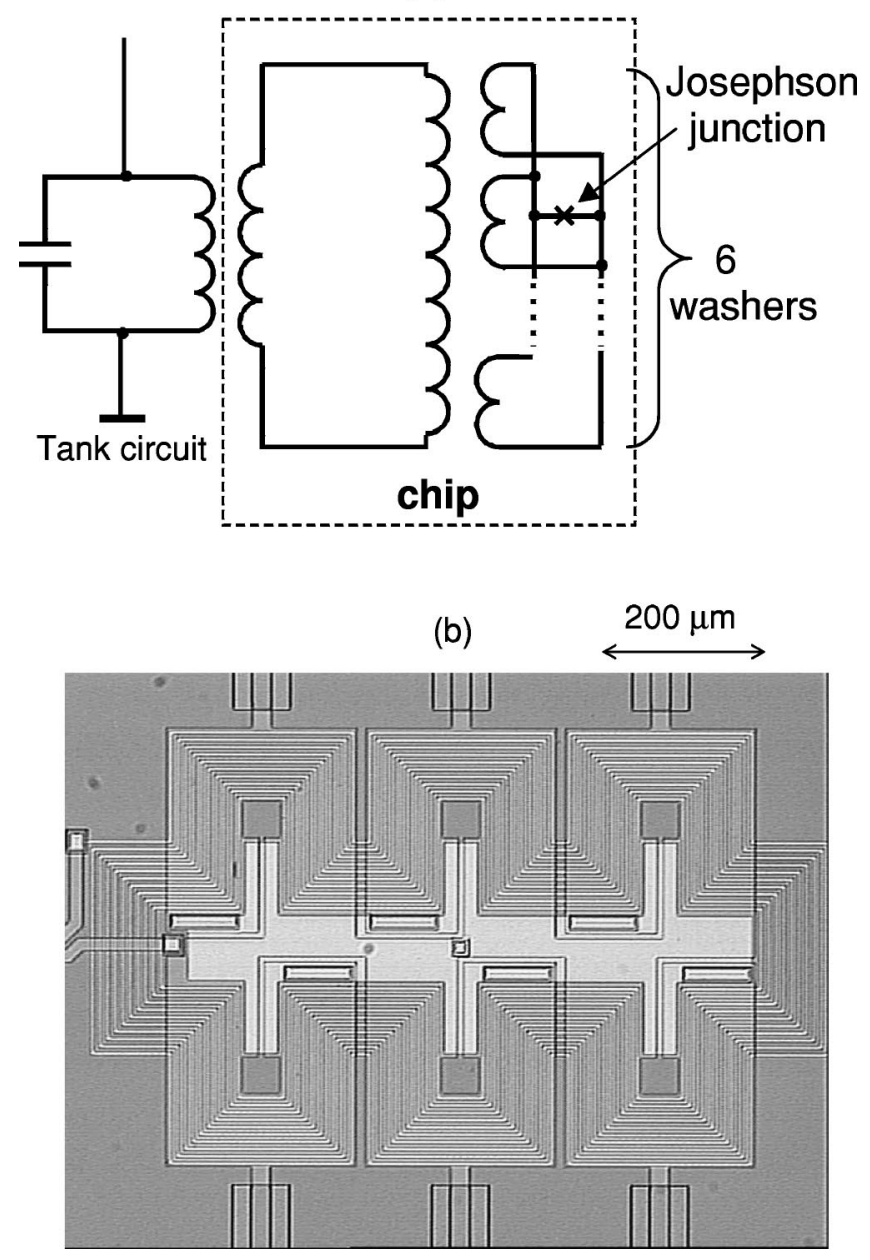

FIG. 31. Nb tunnel junction: (a) circuit and coupling scheme: (b) photo of the six-washer structure. Adapted from Il'ichev, Zakosarenko, Fritzsch, et al., 2001.

$I_{S}(\varphi)$. For this purpose $\mathrm{Nb}$ interferometers with conventional $\mathrm{Nb} / \mathrm{AlO}_{x} / \mathrm{Nb}$ tunnel junctions are used for the calibration.

As follows from the inequality (163), in order to obtain the CФR in the complete phase range for the $f(\varphi)$ $=\sin \varphi$ the regime $\beta<1$ is required. For a junction with critical current of the order of $20 \mu \mathrm{A}$ this leads to a restriction on the interferometer inductance of $L$ $<16 \mathrm{pH}$. Such a small value of $L$ limits the size of the interferometer loop to below $10 \times 10 \mu \mathrm{m}^{2}$. For conventional tank coils with diameter of about $1 \mathrm{~mm}$ it is difficult to get reasonable $M$ values between a tank circuit and an interferometer for a flip-chip arrangement. Moreover, the external magnetic field, producing one flux quantum in the interferometer loop, simultaneously produces the finite value of the magnetic flux in the junction (the size of the tunnel junction is 3.5 $\times 3.5 \mu \mathrm{m}^{2}$ ), suppressing the critical current value due to $I_{C}(H)$ dependence (Il'ichev, Schultze, et al., 2000). In order to avoid these difficulties, the value of $L$ was decreased by using a series of samples with the interferometer loop consisting of six washers, each having an inductance of $55 \mathrm{pH}$, and all connected in parallel. The
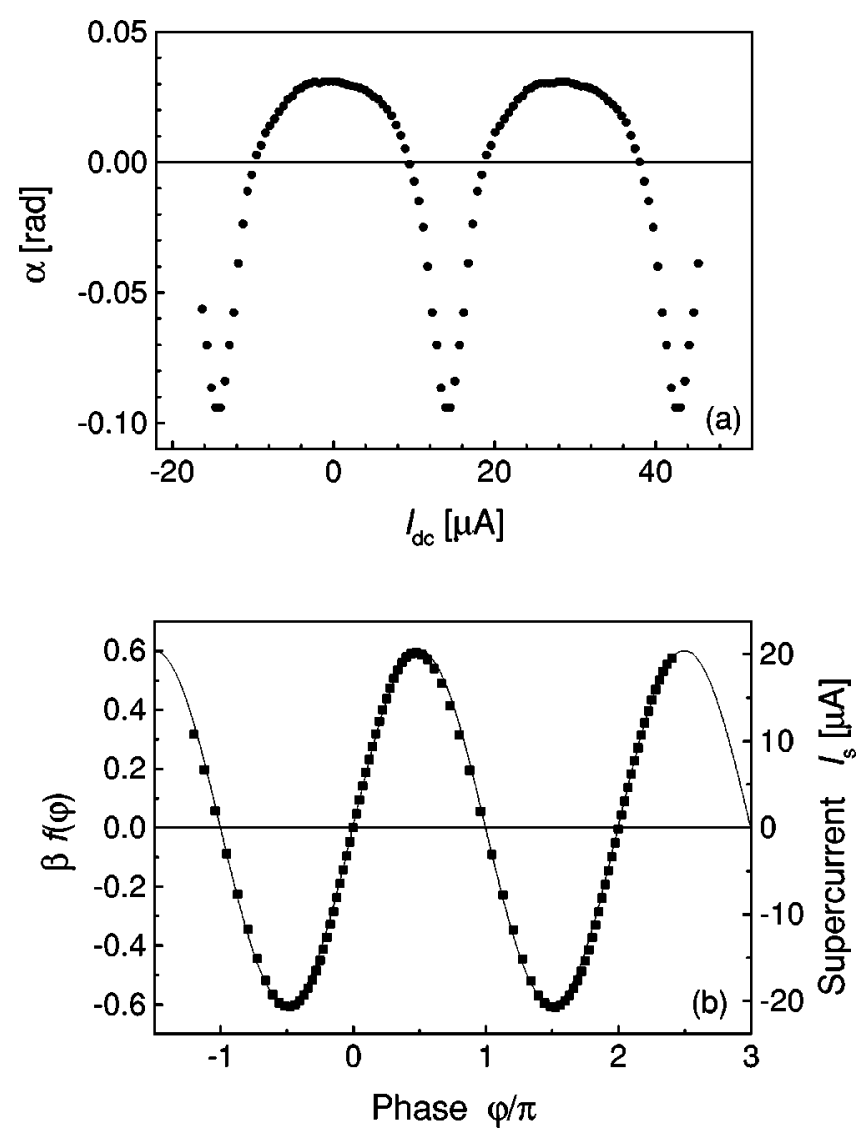

FIG. 32. Current-phase relation and dc current: (a) phase angle between the driving current and the output voltage as a function of dc current $I_{d c}$; (b) the C $\Phi$ R, determined from the upper curve. Adapted from Il'ichev, Zakosarenko, Fritzsch, et al., 2001.

ten-turn coupling coils were placed on each washer and connected in series (see Fig. 31). The coupling coil was connected to a large $5 \times 5-\mathrm{mm}^{2}$ loop integrated on the chip. In this configuration the total inductance of the interferometer was evaluated to be $9 \mathrm{pH}$.

An example of the experimental data obtained for the structure presented in Fig. 31 is shown in Fig. 32. From the measured $\alpha\left(I_{d c}\right)$ dependence (see Fig. 32, upper curve), the corresponding CФR was reconstructed (see Fig. 32, bottom curve). As expected, the sinusoidal CФR was obtained and the critical current was in a good agreement with $I_{C}$ of a similar shunted tunnel junction from the same chip obtained by standard transport measurements.

\section{Measurements of small critical currents}

The properties of supercurrent in submicron-size Josephson junctions are of considerable fundamental and practical interest. Josephson-junction circuits have been recently proposed as qubits (the elementary units in quantum information processing) based either on the flux (Bosco et al., 1997; Ioffe et al., 1999; Mooij et al., 1999) or charge (Averin, 1998; Makhlin et al., 1999) degrees of freedom. Such circuits have recently been suc- 
cessfully tested by Nakamura et al. (1999), Friedman et al. (2000), van der Waals et al. (2000), Yu et al. (2000), Martinis et al. (2002), Vion et al. (2002), and Wallraff et al. (2003). Further practical realization of quantum networks requires comprehensive investigation of quantum coherence in small Josephson junctions.

In SIS tunnel junctions with submicron dimensions the capacitance is reduced to less than $100 \mathrm{fF}$ and $I_{C}$ is typically below $1 \mu \mathrm{A}$, while the normal-state resistance $R_{N}$ is of the order of a few $\mathrm{k} \Omega$. Under these parameters the Josephson coupling energy is smaller than or comparable to the thermal energy $k_{B} T$, and it becomes difficult to distinguish the critical current from the switching current on the $I-V$ curve. The routine solution requires drastically increased cooling efforts as well as careful electromagnetic filtering (Vion et al., 1995).

Il'ichev et al. (Il'ichev, Hoenig, et al., 2001; Il'ichev, Zakosarenko, Fritzsch, et al., 2001) demonstrated that CФR measurements are much less sensitive to thermal fluctuations. In this work, in particular, double-junction samples consisting of two SIS contacts in series were investigated. The values of critical currents were chosen in the range $E_{J} / k_{B} T=0.3-20$. In this case thermal noise substantially affects the switching current measured by the standard $I-V$ method.

For these samples, the experimentally measured CФR's have the shape given by Eq. (117). For highly asymmetric double junctions the shape was close to $\sin \varphi$ as expected. The most remarkable result of this experiment was the demonstration of the ability to resolve the CФR even though the thermal energy exceeded the Josephson coupling energy by a factor of 3.3. Further, in accordance with Eq. (117), the CФR in the symmetric case was far from $\sin \varphi$ and was fitted well by $\kappa \approx 0.4$. From this fitting parameter and the overall critical current of the sample, the individual critical currents 1.1 and $2.8 \mu \mathrm{A}$ were found.

Besides the high resolution, another promising feature of the rf-readout technique was the demonstration that a broad range of supercurrents could be investigated. At the present level, it is possible to reconstruct the CФR from samples characterized by $0.005<\beta<1$, i.e., for fixed loop inductance the CФR can be studied over a range of critical currents that varies by more than two orders of magnitude.

\section{E. Measurement of barrier transparency asymmetry in SINIS junctions}

Cassel et al. (2001), Nevirkovets, Ketterson, and Rowell (2001), and Tolpygo, Brinkman, et al. (2003) have recently shown that one of the hidden difficulties for implementation of SINIS double-barrier junction technology is asymmetry in the barrier transparency of interfaces.

For junctions with relatively small critical current density, e.g., for Josephson voltage standards, intrinsically shunted junctions with low asymmetry have been

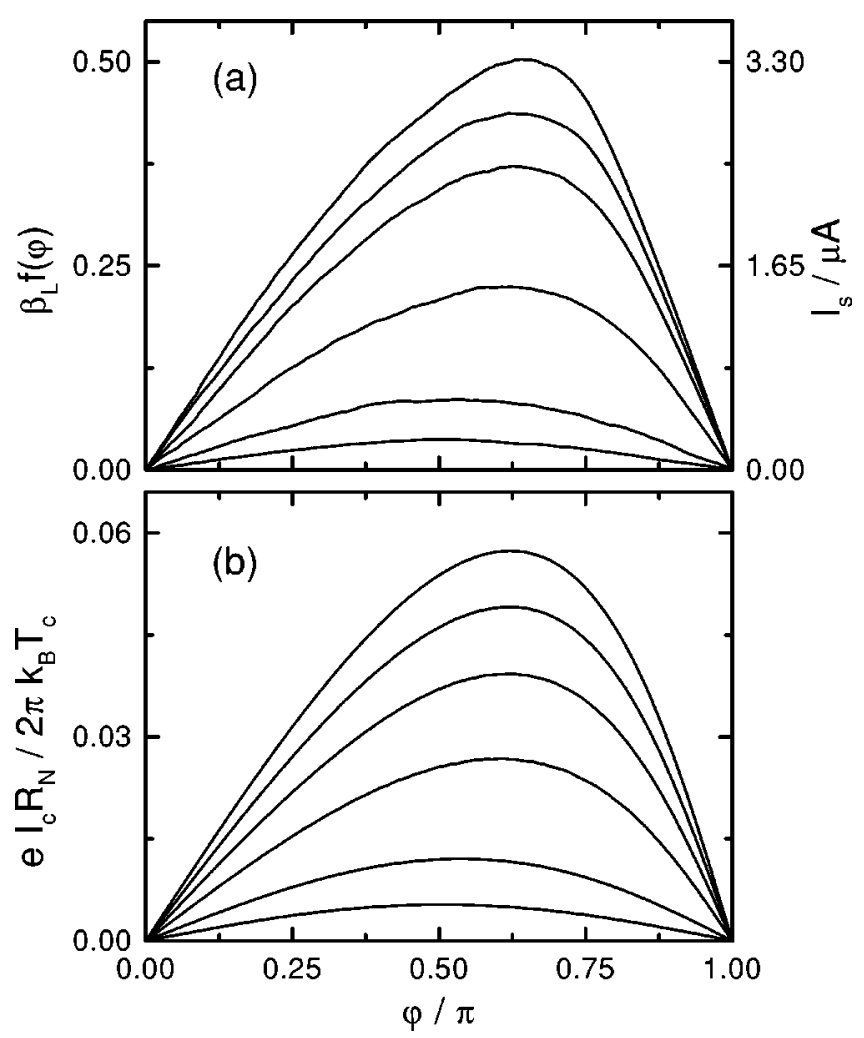

FIG. 33. The CФR of a SINIS junction in the temperature range 1.90-1.95 $\mathrm{K}$ (from top to bottom increasing in steps of $0.01 \mathrm{~K}$ ): (a) experimental results; (b) calculated curves. Adapted from Götz, Grajcar, et al., 2000.

fabricated. ${ }^{10}$ However, for digital applications, junctions with critical current density in the range $1-10 \mathrm{kA} / \mathrm{cm}^{2}$ are required. This may be achieved by shorter oxidation of both $\mathrm{Al}$ layers in a $\mathrm{NbAl} / \mathrm{AlO}_{x} / \mathrm{Al} / \mathrm{AlO}_{x} / \mathrm{Nb}$ structure. The inherently different deposition conditions for the first and second $\mathrm{Al}$ films in multilayer fabrication result in different morphologies of the two Al films. Therefore oxidation performed under the same oxidation conditions should lead to the formation of $\mathrm{AlO}_{x}$ barriers having different transparency (see Cassel et al., 2001; Tolpygo, Brinkman, et al., 2003). The barrier asymmetry reduces the suppressed $I_{C} R_{N}$ product of SINIS junctions and strongly limits their applicability in digital curcuits.

Cassel et al. (2001) have shown that among the different methods for monitoring asymmetry, measurement of the CФR should provide the most accurate results. In accordance with Eqs. (108) and (110), the CФR in SINIS junctions depends on the asymmetry parameter $\gamma_{-}$and the ratio $T_{c}^{\prime} / T$, where $T_{c}^{\prime}$ is the critical temperature of an $\mathrm{Al}$ interlayer. At $T>T_{c}^{\prime} \mathrm{Al}$ is in the normal state, and $I_{S}(\varphi)$ is close to $\sin \varphi$ if $\gamma_{\text {eff }}$ is large. At $T<T_{c}^{\prime} \mathrm{Al}$ is in

\footnotetext{
${ }^{10}$ See Maezawa and Shoji, 1997; Sugiyama et al., 1997, 1999; Balashov et al., 1998, 1999, 2000, 2001; Schulze et al., 1998, 2000; Behr et al., 1999; Buchholz and Kessel, 1999; Khabipov et al., 1999, 2002a, 2002b; Buchholz et al., 2001; Cassel et al., 2001; Kohlmann et al., 2001, 2002; Kieler et al., 2002.
} 


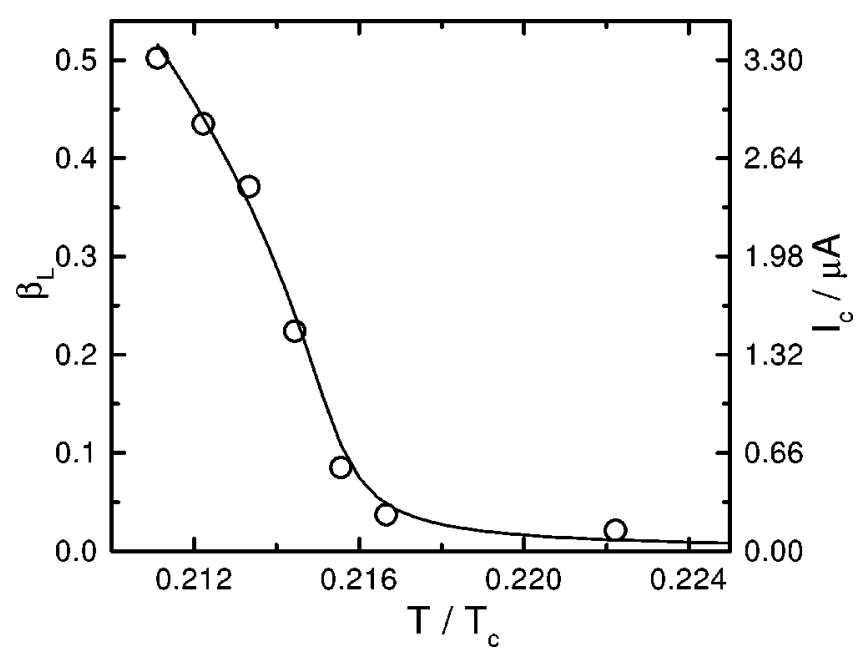

FIG. 34. The fit of the temperature dependence of the critical current. The solid line represents the calculation and symbols are the experimental data. Adapted from Götz, Grajcar, et al., 2000.

the superconducting state, sequential tunneling dominates the supercurrent flow, and the CФR transforms to that given by Eq. (117).

Götz, Grajcar, et al. (2000) confirmed the above considerations by measuring the CФR in SINIS junctions, fitting the data by curves calculated from Eqs. (108) and (110). The set of measured CФRs is presented in Fig. 33(a). The corresponding theoretical curves are shown in Fig. 33(b). As was reported in detail by Götz, Grajcar, et al. (2000), the CФR shown in Fig. 33(a) as well as the $I_{C}(T)$ dependence shown in Fig. 34 can be fitted with a single set of parameters. This procedure allows one to extract all the junction characteristics for this series of samples, namely, $T_{c}^{\prime} \approx 1.935, \gamma_{\mathrm{eff}} \approx 10^{5}, R_{N} \approx 83 \Omega$, and $\gamma_{-} \approx 0.45$.

This method opens the way for calibration of the second oxidation process in SINIS junction technology, based on accurate measurement of the barrier asymmetry parameter $\left(\gamma_{-}\right)$.

\section{F. Current-phase relation in hybrid S-2DEG-S junctions}

For low-temperature electronics it is quite natural to combine the technology of modern superconductors with that of semiconductors. One of the practical realizations of such a combined approach is to couple two superconducting electrodes by a 2D electron gas (Marsh et al., 1994; Takayanagi et al., 1995a; Chrestin et al., 1997, 1999; Heida et al., 1999; Lehnert et al., 1999; Neurohr et al., 1999; Grajcar, Ebel, et al., 2002; Schäpers et al., 2003).

For practical applications it is important to estimate the degree to which the critical current is limited by the S-2DEG interfaces. Grajcar, Ebel, et al. (2002) have demonstrated that the CФR measurements may provide this information.

Grajcar and co-workers (Grajcar, Ebel, et al., 2002; Grajcar, Hlubina, et al., 2002) studied samples consisting

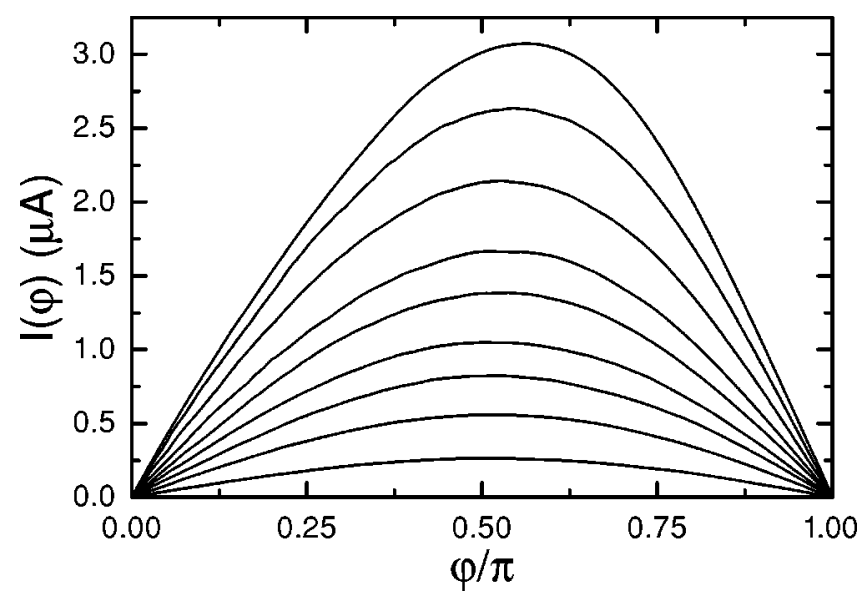

FIG. 35. The current-phase relation in a $\mathrm{Nb} / \mathrm{InAs}(2 \mathrm{DEG}) / \mathrm{Nb}$ Josephson junction. The curves from top to bottom correspond to $T=2.75,3,3.25,3.5,3.75,4,4.2,4.5$, and $5 \mathrm{~K}$. Adapted from Grajcar, Ebel, et al., 2002.

of two $\mathrm{Nb}$ electrodes coupled by a $2 \mathrm{DEG}$ formed in the native inversion layer at the surface of $p$-type bulk InAs (see Chrestin et al., 1997 for details). The electron density and the Fermi velocity of the 2DEG in the channel could be adjusted via the field effect using a gate electrode. At a typical electron density of $n_{s}=1.2$ $\times 10^{12} \mathrm{~cm}^{-2}$ and mobility $\mu \approx 10000 \mathrm{~cm}^{2} / \mathrm{V} \mathrm{s}$, the electron mean free path, $\ell \approx 240 \mathrm{~nm}$, was comparable to the coherence length $\xi_{2} \approx 145 \mathrm{~nm}$ at $1.8 \mathrm{~K}$ and the channel length $d \approx 120 \mathrm{~nm}$. Thus the studied quasiballistic junction was in the intermediate regime between short and long weak-link regimes. Due to proximity effect, a thin InAs layer underneath the $\mathrm{Nb}$ electrodes is superconducting as well, resulting in formation of a SIN-2DEGNIS junction.

To describe theoretically the Josephson effect in such a structure, a more general expression than that discussed in Secs. III and IV is needed. It should take into account both the scattering of electrons inside the 2DEG and the proximity effect in the NS electrodes. The expression for the supercurrent was derived by Grajcar, Ebel, et al. (2002):

$$
\begin{aligned}
\frac{e I R_{0}}{2 \pi T_{c}} & \\
= & \frac{8 T}{T_{c}} \sum_{n=0}^{N-1} \sum_{\omega}-a^{2}\left|S_{12}\right|^{2} \sin (\varphi) \\
& \times \frac{\left.S_{11}^{2}\right]+a^{4}\left|S_{11}^{2}+S_{12}^{2}\right|^{2}}{1-2 a^{2}\left[\left|S_{12}\right|^{2} \cos (\varphi)+\mid S^{2}\right.}
\end{aligned}
$$

and combines the result of Brouwer and Beenakker (1997) for $I_{S}(\varphi)$ in chaotic Josephson junctions with the constriction model developed by Aminov et al. (1996) and Golubov et al. (1997). Here $R_{0}=h / e^{2}$ is the resistance quantum, $a$ is the amplitude of the Andreev reflection at the InAs(2DEG)/InAsNb NIS interface, $S_{i j}$ are the elements of the scattering matrix for electron 
propagation across the structure, and $N$ is the number of open transmission channels.

Experimental CФR's are shown in Fig. 35. Gradual deviations from sinusoidal behavior with decreasing temperature can be clearly seen. Reasonable agreement between experimental and theoretical data was obtained [for details see Grajcar, Ebel, et al. (2002)].

We should point out that a relatively large inductance $(80 \mathrm{pH})$ for the interferometers was used in this set of experiments. In this case the inequality (163) requires that the current be smaller than $4 \mu \mathrm{A}$. Usually the critical current of a $\mathrm{Nb} / \mathrm{InAs}(2 \mathrm{DEG}) / \mathrm{Nb}$ Josephson junction exceeds this value considerably. The junctions measured by Grajcar, Ebel, et al. (2002) had reduced critical current and lower interface transparency. Still, a shift of the maximum of the CФR towards $\pi$ was observed. The investigation of the CФR of junctions with higher critical current requires a more sophisticated technique.

\section{G. Current-phase relation anomalies in high- $T_{C}$ superconducting junctions}

\section{1. $d$-wave effects in high- $T_{c}$ superconducting}

The most important phenomenological difference between the high- $T_{c}$ superconducting cuprates and conventional superconductors is the orbital symmetry of the superconducting order parameter. In high- $T_{c}$ superconductors the pair potential changes sign depending on the direction in momentum space according to $\Delta(\theta)$ $=\Delta_{0} \cos (\theta-\alpha)$, where $\theta$ is the angle between the $x$ axis and the wave vector and $\alpha$ is the $\mathrm{Cu}-\mathrm{Cu}$ bond direction of the superconductor. As a result, the $\pi$ shift of the superconducting phase occurs between the $a$ and $b$ crystallographic directions, The unconventional symmetry in cuprates was experimentally confirmed by phasesensitive experiments (see van Harlingen, 1995; Tsuei and Kirtley, 2000).

Several comprehensive review articles exist on the Josephson effect in high- $T_{c}$ superconducting junctions, both on fundamental aspects and an applications [see van Harlingen (1995), Sigrist and Rice (1995), Delin and Kleinsasser (1996), Kashiwaya and Tanaka (2000), Tsuei and Kirtley (2000), Hilgenkamp and Mannhart (2002)]. The theory of the effects of $d$-wave symmetry on the CФR was discussed in detail in review papers by Kashiwaya and Tanaka (2000) and Löfwander et al. (2001).

There are several important consequences of an unconventional order-parameter symmetry in high- $T_{c}$ superconducting junctions. First, in high- $T_{c}$ electrodes the superconducting state in the vicinity of the interfaces differs essentially from that in the bulk. Due to the angular dependence of the pair potential, incoming and reflected quasiparticles at certain trajectories through the junction probe a sign change of the pair potential upon reflection. This sign change has fundamental consequences-it leads to the appearance of robust midgap states at zero energy ( $\mathrm{Hu}, 1994)$. The midgap states do not occur at the sign-conserving trajectories, but in both cases the interface is pair breaking, and the self- consistently determined magnitude of the pair potential near the interface is reduced in comparison with its bulk value. The nucleation of an order parameter with different symmetry is possible, as well.

Different types of high- $T_{c}$ Josephson junctions have been fabricated, including ramp-type junctions (Gao et al., 1990), grain-boundary junctions (see reviews by Tsuei and Kirtley, 2000; Hilgenkamp and Mannhart, 2002), intrinsic junctions (Kleiner and Müller, 1994; Krasnov et al., 2000, 2001; Latyshev et al., 2001), combination high- $T_{c}$ /low- $T_{c}$ junctions (van Harlingen, 1995; Smilde et al., 2002), and SNS-type junctions (Delin and Kleinsasser, 1996).

The physics of the Josephson effect in high- $T_{c}$ junctions is strongly influenced by a number of physical processes. They are

(a) robust midgap states at zero energy, which may contribute to the supercurrent (see $\mathrm{Hu}, 1994$; Tanaka and Kashiwaya, 1995, 1996; Barash et al., 1996; Fogelström et al., 1997; Riedel and Bagwell, 1998; Barash, 2000);

(b) bound states with finite energy due to suppression of the pair potential at the interface (see Barash et al., 1997);

(c) a subdominant order parameter with different symmetry from that in the bulk (see Fogelström et al., 1997);

(d) gapless singlet superconductivity nucleated at rough interfaces (see Golubov and Kupriyanov, 1998, 1999a, 1999b);

(e) spontaneous half-flux vortices (Mannhart et al., 1996; Tsuei and Kirtley, 2000; Hilgenkamp et al., 2003), which may have peculiar static and dynamic properties (Goldobin et al., 2002).

The possibility of broken time-reversal symmetry in cuprates, when the order parameter in the bulk has an imaginary subdominant component, was suggested by Sigrist and Rice (1995). The consequences for high- $T_{c}$ superconductor junctions have been discussed and some experimental findings have been interpreted as realizations of this state (for references see Tanaka and Kashiwaya, 2000; Tsuei and Kirtley, 2000; Löfwander et al., 2001). Further, it was predicted by Löfwander et al. (2000) and Amin et al. (2001) that spontaneous timereversal symmetry breaking at high- $T_{c}$ junctions may occur even in the absence of subdominant components in the bulk. Note that, according to Geshkenbein et al. $(1986,1987)$, the symmetry property of the CФR, Eq. (4), should be violated when time-reversal symmetry is broken.

So far, the experimental data, including the CФR measurements discussed below, have not been able to resolve unambiguously the issue of time-reversal symmetry breaking. Further study is needed to realize the full potential of the CФR measuring technique in this respect.

Here we shall concentrate on experimental measurements of the CФR in high- $T_{c}$ superconductor junctions, where the anomalies due to pure $d$-wave symmetry have 
been revealed by C $\Phi \mathrm{R}$ measurements: (1) detection of a strong second harmonic in the CФR, (2) manifestation of midgap states and (3) CФR anomalies in $c$-axis tunneling.

\section{Asymmetric $45^{\circ}$ grain-boundary junction}

Tanaka (1994) and Yip (1995) pointed out that the Josephson coupling energy may have minima neither at $\varphi=0$ nor at $\varphi=\pi$ in a junction between pure $d$-wave superconductors, even in the absence of time-reversal symmetry breaking. This may occur due to strong reduction of the first harmonic $I_{1}$ in Eq. (7) so that the second harmonic $I_{2}$ may become comparable to or larger than $I_{1}$.

Walker and Lüttmer-Strathmann (1996) took these arguments further. Since the order parameter is bound to the crystal lattice, the supercurrent of a junction between two $d$-wave superconductors depends on the orientation of the $d$-wave electrodes with respect to their boundary. Let $\theta_{1}$ and $\theta_{2}$ denote the angle between the normal to the junction interface and the $a$ axis in electrodes 1 and 2 . The symmetry of the problem dictates that (Walker and Lüttmer-Strathmann, 1996)

$$
I_{1}=I_{C} \cos 2 \theta_{1} \cos 2 \theta_{2}+I_{S} \sin 2 \theta_{1} \sin 2 \theta_{2} \text {. }
$$

The coefficients $I_{C}$ and $I_{S}$ are functions of the barrier strength and temperature. As follows from Eq. (167), the criterion for the observation of an anomalous $I_{S}(\varphi)$, $I_{1}=0$, is realized for an asymmetric $45^{\circ}$ junction, i.e., a junction with $\theta_{1}=45^{\circ}$ and $\theta_{2}=0$. In this case one expects double-periodic $I_{S}(\varphi)$ dependence. Note that such a type of CФR was discussed by Ioffe et al. (1999) in their proposal for a $d$-wave qubit.

Before discussing the detection of the second CФR harmonic, let us mention that in practice the properties of grain boundaries are strongly influenced by the effect of meandering (faceting) of the junction boundary (Copetti et al., 1995; Hilgenkamp et al., 1996). Within the $d$-wave scenario, faceting has a more significant influence on the electronic properties of boundaries with a misorientation close to $45^{\circ}$ than on boundaries with considerably smaller misorientation angles (Hilgenkamp et al., 1996). Grain-boundary junctions, fabricated on bicrystal substrates with the misorientation angle $45^{\circ}$, contain a higher density of facets which by themselves show anomalous behavior. Due to the sign difference of the adjacent lobes of the $d$-wave order parameter, many facets are biased with an additional $\pi$ phase shift ( $\pi$ facets). Therefore the supercurrent density $J_{S}$ is a random alternating function in the plane of the contact. This effect must be taken into account in the interpretation of the data.

Il'ichev and co-workers (Il'ichev, Zakosarenko, IJsselstein, et al., 1998a, 1998b, 1999b) measured the CФR in $45^{\circ}$ grain-boundary junctions with $\theta_{1,2}=22.5^{\circ}$ as well as $\theta_{1}=19^{\circ}$ and $\theta_{2}=26^{\circ}$. The junction size was between 1 and $3 \mu \mathrm{m}$. These junctions exhibit deviations of the CФR from the conventional sinusoidal form. The difference between first and second harmonics of the CФR

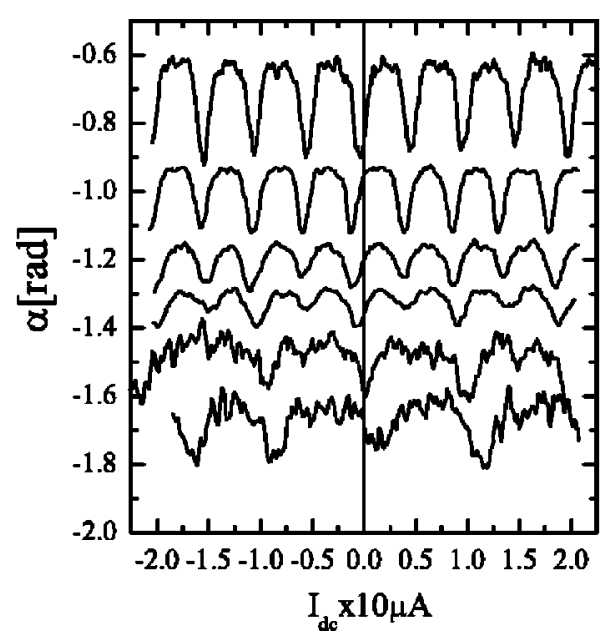

FIG. 36. Phase angle $\alpha$ as a function of the dc current flowing through the tank coil for an interferometer with an asymmetric $45^{\circ}$ grain-boundary junction at various temperatures. Adapted from Il'ichev et al., 1999a.

was not too large, $I_{2} / I_{1} \sim 0.1-0.2$. But surprisingly the second harmonic had a positive sign, $I_{2}>0$. This means that the supercurrent reaches its maximum value at $\varphi_{\max }<\pi / 2$. As discussed in Sec. IV.D, such a CФR may be realized in an $s$-wave SIS junction with high barrier transparency due to depairing by the current (Kupriyanov, 1992). However, high barrier transparency is hardly the case in $45^{\circ}$ grain-boundary junctions. To our knowledge, the observed CФR with $\varphi_{\max }<\pi / 2$ cannot be explained in the framework either of the $s$-wave or the $d$-wave scenario on the assumption that the junction has a flat interface.

Il'ichev, Zakosarenko, IJsselstein, et al. (1999a) applied the model developed by Mints (1998) for a faceted junction to derive the C $\Phi R$ of a Josephson junction in the presence of a randomly distributed alternating current density, assuming that the local C $\Phi R$ was sinusoidal. It was shown that the model was in good agreement with experimental results.

In the other set of measurements by Il'ichev et al. (1999b), the CФR of a grain-boundary junction $\left(\theta_{1}\right.$ $=0^{\circ}$ and $\theta_{2}=45^{\circ}$, the so-called asymmetric grainboundary junction) 1-2 $\mu \mathrm{m}$ size were measured. It was found that $\left|I_{2} / I_{1}\right|>1$ with a negative second harmonic. The results of measurements at different temperatures are shown in Fig. 36. At $T=15$ and $20 \mathrm{~K}$, the curves are $2 \pi$ periodic, but the local minima show the presence of the second harmonic of the CФR. At $T<10 \mathrm{~K}$ the amplitude of the second harmonic becomes larger than the first.

The CФR determined from the data in Fig. 36 is shown in Fig. 37. For all curves, a minimal necessary shift consistent with $I_{S}(\varphi=0)=0$ was performed. Thus it was assumed that at $\varphi_{d c}=0$ a minimum of $\alpha\left(\varphi_{d c}\right)$ was realized. For an interferometer with a conventional $s$-wave weak link, at $\varphi_{d c}=0$ a maximum of $\alpha\left(\varphi_{d c}\right)$ is realized. Note that the maximum of $\alpha\left(\varphi_{d c}\right)$ at $\varphi_{d c}=0$ implies a diamagnetic response, while a minimum there implies a paramagnetic response of the interferometer in the limit of small applied fields. Figure 38 shows the 


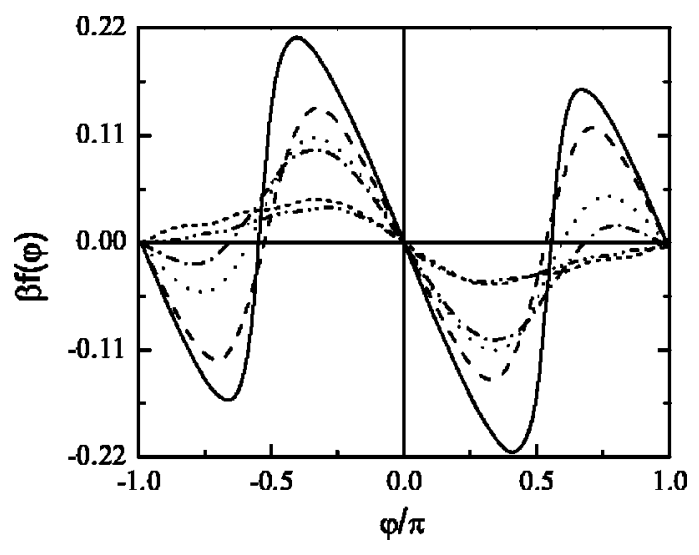

FIG. 37. Josephson current through a junction as a function of the phase difference $\varphi$, determined from the data in Fig. 36. Adapted from Il'ichev et al., 1999a.

coefficients $I_{1}$ and $I_{2}$ determined by Fourier analysis of the CФR at various temperatures. With decreasing $T$, $\left|I_{2}\right|$ grows monotonically down to $T=4.2 \mathrm{~K}$, while the $I_{1}$ component exhibits only a weak temperature dependence.

In order to explain the fact that $\left|I_{2} / I_{1}\right|>1$ within the faceted scenario, the alternating current density should average to zero (Hlubina et al., 2003). This is possible only for sufficiently long junctions, at least much longer than the Josephson penetration depth, whereas in the experiment of Il'ichev, Zakosarenko, IJsselstein, et al. (1999a) much shorter junctions with $L \sim 1 \mu \mathrm{m}$ were studied. Therefore it is impossible to explain the large measured $I_{2} / I_{1}$ ratio within the faceted scenario. One explanation might be that for some reason only a few facets carry the supercurrent, and therefore averaging does not occur. In this case the models of Tanaka (1994) and Walker and Lüttmer-Strathmann (1996) for the flat interface are applicable and can explain the results.

In order to avoid the influence of faceting, submicron junctions are required. These results will be discussed in the next section.

\section{Symmetric $45^{\circ}$ grain-boundary junction: Manifestation of} midgap states in the current-phase relation

The presence of midgap states is expected to influence in a spectacular way Josephson junctions between

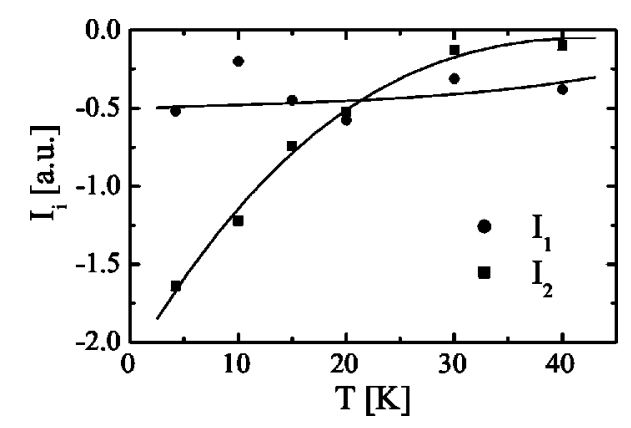

FIG. 38. Temperature dependence of the Fourier expansion of the CФR, determined from the data in Fig. 36. Adapted from Il'ichev et al., 1999a.

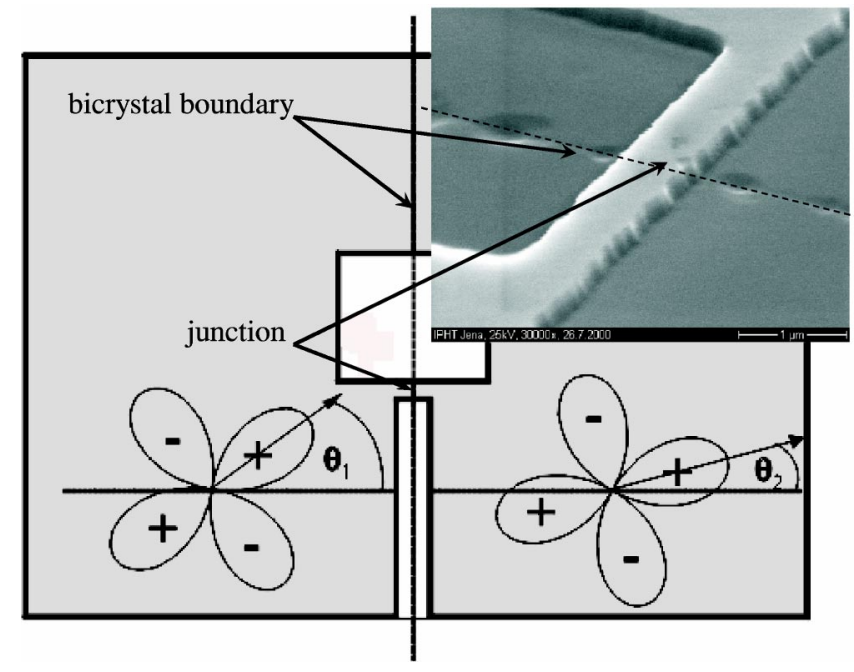

FIG. 39. Schematic picture of an interferometer. The YBCO thin film occupies the gray area. The inset shows an electron microscope image of the grain-boundary Josephson junction. Adapted from Il'ichev, Grajcar, et al., 2001.

$d$-wave superconductors with different crystallographic orientations. As shown by Tanaka and Kashiwaya (1995, 1996), Barash et al. (1996), Fogelström et al. (1997), Riedel and Bagwell (1998), and Barash (2000), the midgap states in symmetric $d$-wave junctions provide an anomalous contribution to the supercurrent with opposite sign to the contribution of the regular Andreev bound states. As a result, a sign change of the first CФR harmonic $I_{1}$, i.e., a crossover to a $\pi$ junction, should take place at low $T$.

Motivated by the search for the midgap states, Il'ichev, Grajcar, et al. (2001) have studied symmetric grain-boundary junctions $\left(\theta_{1}=\theta_{2}=22.5^{\circ}\right)$ of small submicron size. It is important to note that in this case standard transport measurements of $I_{C}$ are only possible at low temperatures, when the thermal energy $k_{B} T$ is smaller than the Josephson coupling energy. As was described above, the radio-frequency method offers the possibility of investigating $I_{S}(\varphi)$ at thermal energy much higher than the Josephson coupling energy.

A temperature-controlled crossover to a $\pi$ junction should manifest itself as a $\pi$ shift of the phase $\alpha$-vs- $I_{d c}$ oscillations. For a junction with nominal width of about $0.5 \mu \mathrm{m}$, as shown in Fig. 39, such behavior was observed experimentally (see Fig. 40). At high temperatures the curves are $2 \pi$ periodic, but the local minima show the presence of the second harmonic of the CФR. At $T$ $\approx 11 \mathrm{~K}$ the $\alpha$-vs- $I_{d c}$ oscillations are $\pi$ periodic, i.e., the amplitude of the first harmonic equals zero. At lower temperatures the local minima transform to the local maxima, hence the amplitude of the first harmonic changes sign. The corresponding CФR and $I_{C}(T)$ dependence are shown in Figs. 41 and 42, respectively.

Note also the remarkably large magnitude of the second harmonic $I_{2}$. As was argued by Il'ichev, Grajcar, et al. (2001), this enhancement may also be explained by the contribution of the midgap states. An alternative model which explains the large second harmonic by 


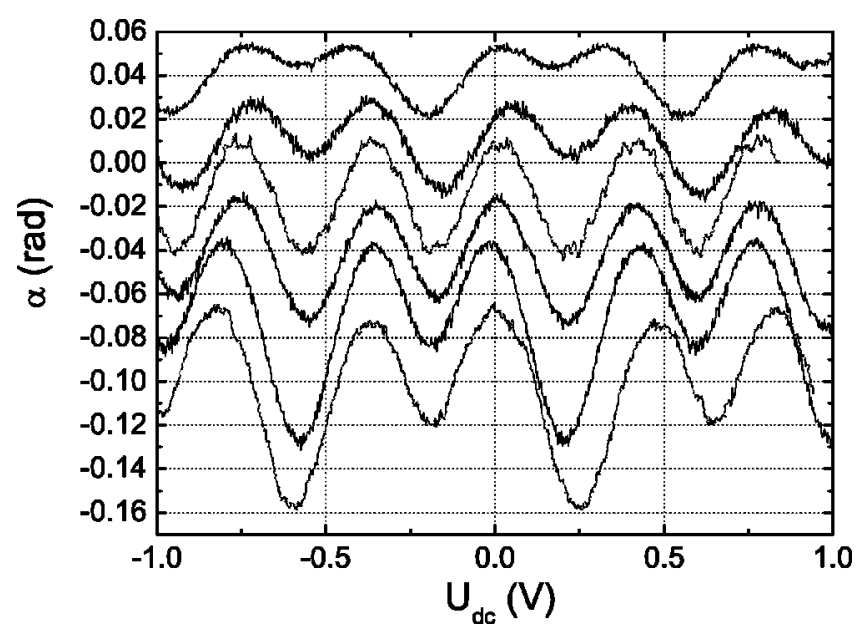

FIG. 40. The phase angle $\alpha$ as a function of the dc current flowing through the tank coil for an interferometer with a submicron symmetric $45^{\circ}$ grain-boundary Josephson junction at various temperatures. From top to the bottom the data correspond to $T=20,15,11,10,5$, and $1.6 \mathrm{~K}$. Adapted from Il'ichev, Grajcar, et al., 2001.

quantum fluctuations of the order parameter was proposed by Hlubina (2002; Hlubina et al., 2003).

We conclude that the data for submicron grainboundary junctions are in qualitative agreement with theoretical predictions for the CФR for a high- $T_{c}$ junction with ideally flat interfaces. Thus the experiment by Il'ichev, Grajcar, et al. (2001) has revealed the contribution of the midgap states to the Josephson effect in high$T_{c}$ superconducting junctions.

\section{4. $c$-axis high- $T_{C}$ superconducting junctions}

Recently, it has become clear that a finite admixture of the order-parameter component with $s$-wave symmetry should exist in addition to the dominant $d$-wave order parameter in YBCO materials. An elegant $c$-axis tunneling experiment by Kouznetsov et al. (1997) has directly shown that the $s$-wave component changes sign upon crossing the twin boundary in YBCO.

The above picture of the YBCO pairing state is challenged by the experimental observation of a finite $c$-axis Josephson current between heavily twinned YBCO and a $\mathrm{Pb}$ counterelectrode (Sun et al., 1996). The contribu-

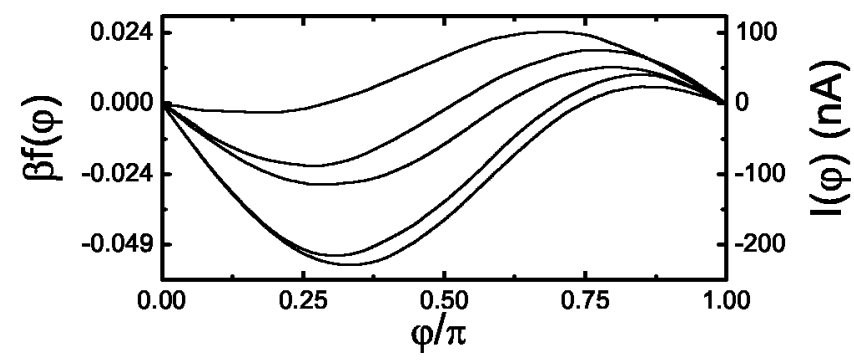

FIG. 41. The CФR for a submicron symmetric $45^{\circ}$ grainboundary Josephson junction, calculated from the data in Fig. 40. From top to the bottom the data correspond to $T=15,11$, 10, 5, and 1.6 K. Adapted from Il'ichev, Grajcar, et al., 2001.

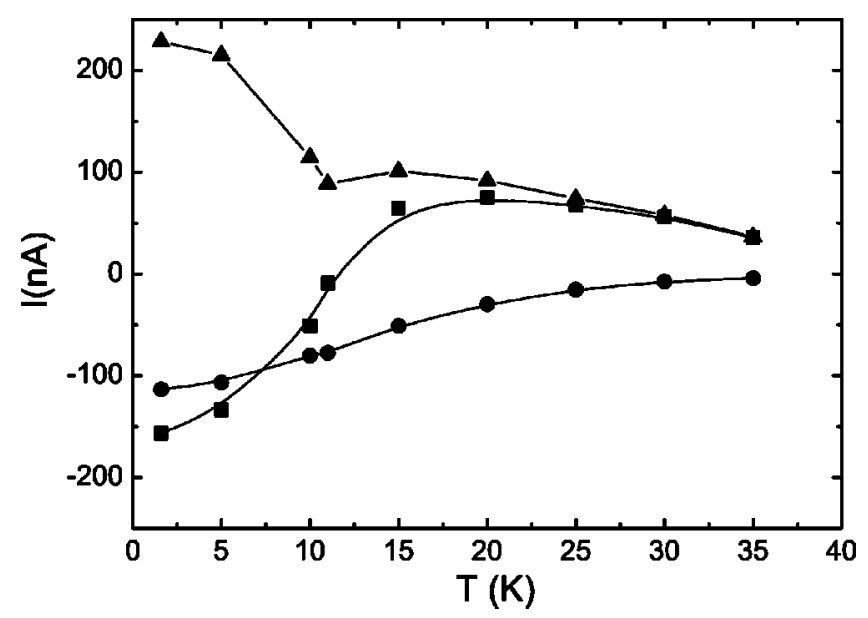

FIG. 42. Submicron $45^{\circ}$ grain-boundary Josephson junction: $\boldsymbol{\Delta}$, critical current; $\boldsymbol{\square}$, the harmonic component $I_{1} ; \boldsymbol{O}$, harmonic component $I_{2}$, all three as a function of temperature as calculated from the data in Fig. 40. Adapted from Il'ichev, Grajcar, et al., 2001.

tion of the $s$-wave part of the YBCO order parameter to the Josephson coupling between $\mathrm{Pb}$ and YBCO should average to zero for equal abundances of the two types of twins. That is, the macroscopic pairing symmetry of twinned YBCO samples should be a pure $d$ wave (Walker and Lüttmer-Strathmann, 1996). Tanaka (1994) has shown that a finite value of the Josephson current occurs for second-order tunneling between YBCO and $\mathrm{Pb}$. However, measurements of microwave-induced steps at multiples of $h f / 2 e$ on the $I-V$ curves of $\mathrm{Pb} / \mathrm{Ag} /$ YBCO tunnel junctions imply dominant first-order tunneling (Kleiner et al., 1996). Therefore the finite $c$-axis Josephson current has to result from a nonvanishing $s$-wave component of the macroscopic pairing state (Walker and Lüttmer-Strathmann, 1996). Two alternative explanations of how this can take place have been discussed in the literature:

(i) Sigrist et al. (1996) suggested that the phase of the $s$-wave component in YBCO does not simply jump from 0 to $\pi$ upon crossing the twin boundary, but rather changes in a smooth way, attaining the value of $\pi / 2$ right at the twin boundary. The twinned YBCO sample is thus assumed to exhibit a macroscopic $d+i$ s pairing symmetry. A related picture has been proposed by Haslinger and Joynt (2000), who propose a $d+i s$ surface state of YBCO.

(ii) O'Donovan et al. (1997) argued that a difference in the abundances of the two types of twins implies a $d$ $+s$ symmetry of the macroscopic pairing state. Let us point out that structural peculiarities of other types (such as a lamellar structure in a preferred direction) may also lead to the $d+s$ macroscopic pairing symmetry.

Komissinski, Il'ichev, Ovsyannikov, Kovtonyuk, et al. (2002) have measured the CФR in $c$-axis $\mathrm{Nb} / \mathrm{Au} /$ (001)YBCO heterojunctions and found a deviation from the sinusoidal shape, as shown in Fig. 43. The obtained results are consistent with the statement that the macroscopic pairing symmetry in YBCO films is of the $d+s$ type, most probably due to a difference in the abun- 


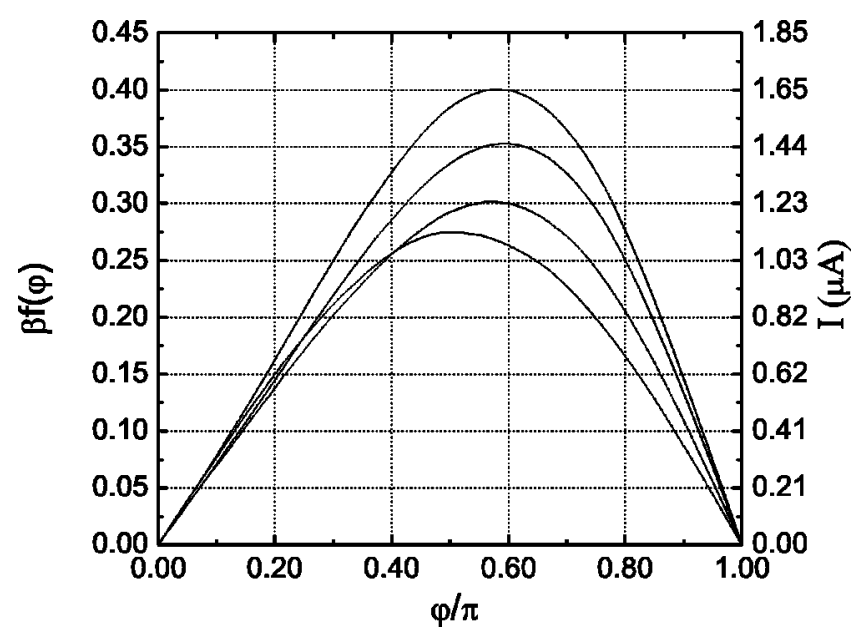

FIG. 43. The CФR for a Nb/Au/(001)YBCO heterojunction ( $c$ contact). From top to the bottom the data correspond to $T$ $=1.66,2.5,4.2$, and $6 \mathrm{~K}$.

dances of the two types of twins. Komissinski, Il'ichev, Ovsyannikov, Kovtonyuk, et al. (2002) used a single set of parameters to explain the measured values of the normal-state resistance $R_{N}$ together with $I_{1}$ and $I_{2}$ within the standard microscopic $d+s$ picture of the YBCO pairing state, under the additional assumption that the barrier fluctuates along the junction.

\section{General remarks}

Due to the relatively complicated structure of high- $T_{c}$ materials, there is no generally accepted picture of the nature of barriers in grain-boundary junctions, where many mechanisms have been suggested [see Hilgenkamp and Mannhart (2002), and references therein]. The CФR measurements of Il'ichev, Zakosarenko, IJsselstein, et al. (1999c) and by Grajcar, Hlubina, et al. (2002) for the $24^{\circ}$ and $36^{\circ}$ grain-boundary junctions are consistent with the tunnel limit prediction that $I_{S}(\varphi)$ $\propto \sin \varphi$. According to the data, a transition from lowangle boundaries to large-angle ones increases the grainboundary resistance (for a detailed theoretical analysis, see Gurevich and Pashitskii, 1998). The average transparency of $45^{\circ}$ is smaller than for low-angle grainboundary junctions. Therefore it is natural to try to explain the large amplitude of the second harmonic of the CФR in the tunnel limit. Recently Hlubina (2003) and Hlubina et al. (2003) proposed to take into account quantum fluctuations of the order parameter in order to explain the experimental data on the C $\Phi \mathrm{R}$ in grainboundary junctions.

The barrier in grain-boundary junctions may also be represented as a disordered normal metal, such that the structure is of the SNS type. This may occur, for example, due to a short coherence length in high- $T_{c}$ superconductors (Deutscher and Müller, 1987), deviations from stoichiometry (Gross and Mayer, 1991; Halbritter, 1993), or band bending (Hilgenkamp and Mannhart, 1998; Nikolic et al., 2002). The generation of the second harmonic in SNS junctions was discussed by Huck et al.
(1997) and Zagoskin (1997). Asano (2001) has studied theoretically the CФR in SNS junctions of unconventional superconductors, including the cases of spinsinglet, spin-triplet unitary, and spin-triplet nonunitary superconductors. This study generalized previous theories by Sigrist and Ueda (1991) and Millis et al. (1988) to the case in which midgap states and disorder are present in the junction. A challenging problem for future C $\Phi R$ measurements remains to verify the predictions of various models in order to shed light on the conductance mechanisms across the grain boundary in high- $T_{c}$ superconductors.

Let us close the experimental part of the review by a general remark concerning method. First measurements of the CФR for high- $T_{c}$ junctions were reported by Tinchev (1994) and Polushkin et al. (1995) using conventional rf technique at temperature close to $T_{c}$, and sinusoidal CФR was observed. Apart from methods for measurements of the CФR, described above, some conclusions about the behavior of the C $\Phi \mathrm{R}$ can be reached by measuring microwave-induced Shapiro steps (Kleiner et al., 1996; Ovsyannikov et al., 1999, 2000; Komissinski, Ovsyannikov, et al. 2001, 2002; Borisenko et al., 2002; Komissinski, Il'ichev, et al., 2002). In this case the junction of interest is biased by finite voltage, which can lead, for instance, to deviations of the electron energy distribution function $f(E)$ from equilibrium. This in turn, according to Eq. (2), generally leads to deviations of the CФR from equilibrium. Therefore in principle information on the CФR can be obtained from Shapiro steps. However, the experimental results should be carefully analyzed for each particular case.

\section{SUMMARY AND OUTLOOK}

We have discussed various possible forms of the CФR in different types of Josephson junctions. Due to space limitations, only a small fraction of the research work in this area was reviewed. We did not consider the possibility of controlling the CФR by high-frequency irradiation or dc current injection into a weak link, nor did we discuss Josephson transistors and multiterminal Josephson devices. Nevertheless, even under these limitations we have demonstrated the existence of a variety of current-phase relations as a result of complex fundamental processes in superconductors.

We have discussed resonant tunneling in SIS structures and the crossover from a classical tunnel junction to a set of parallel constrictions or to a double-barrier structure. In particular, the models described or referred to in this review may serve as a basis for theoretical description of junctions with ultrathin tunnel barriers, which are of great practical interest (Naveh et al., 2000; Rippard et al., 2002).

The type of CФR found in SNS junctions makes it possible to judge the degree of pair breaking in the $\mathrm{S}$ electrodes. This may provide information that is important for practical applications, since pair breaking by current or proximity effect may lead to a reduction in 
the high-frequency cutoff by orders of magnitude compared to a classical SNS junction.

The CФR in ballistic SINIS structures is controlled by the interplay between Andreev bound states and transmission resonances. Götz and co-workers (Götz, Grajcar, et al., 2000; Götz, Khanin, et al., 2000) introduced a procedure for extracting all the parameters of SINIS junctions from the measured CФR. This method opens the way for a calibration of the second oxidation process in SINIS junction technology.

In low-dimensional SINIS structures, the supercurrent oscillates as a function of interlayer thickness or electronic momentum. This effect may be used for the control of supercurrent, as demonstrated in S-2DEG-S junctions by Takayanagi et al. (1995a). On the other hand, this effect also has an uncontrollable influence on junction parameters, as was shown by Gudkov et al. (1988).

In Josephson junctions with a magnetoactive interlayer material it is possible to invert the sign of the classical CФR and realize a so-called $\pi$ junction. Moreover, modifications of the CФR are generally not reduced to the $0-\pi$ transition. The energy-phase relation in such junctions may have two minima, at $\varphi=0$ and $\varphi=\pi$. The effect is observable in a variety of structures: SFS sandwiches or SFcFS constrictions using metallic ferromagnets, tunnel junctions with a ferromagnetic insulator, or junctions with magnetically active interfaces. These phenomena may be used in the engineering of cryoelectronic devices manipulating spin-polarized electrons and in qubit structures.

For a long time, the real shape of the CФR was not considered as an important factor. Practical Josephsonjunction-based devices have been implemented on tunnel SIS structures with $I_{S}(\varphi) \propto \sin \varphi$. However, these devices have relatively low critical current densities and require external shunting by a normal resistor to avoid undesirable hysteresis on current-voltage characteristics. These drawbacks limit the integration scale of the devices and their application in the $\mathrm{mK}$ temperature range, e.g., in quantum-bit readout systems. The new concept of a programmable voltage standard (Hamilton et al., 1995) essentially employs junctions with a nonhysteretic current-voltage characteristic and large current densities. Thus the transition from classical $\mathrm{Nb} / \mathrm{Al} / \mathrm{AlO}_{x} / \mathrm{Nb}$ tunnel junctions to intrinsically shunted high- $J_{C}$ tunnel junctions, double-barrier structures, or SNS weak links seems to be very natural for the future development of basic elements in large-scale integrated superconducting circuits.

As was shown in this review, the CФR in all these structures differs from $I_{S}(\varphi) \propto \sin \varphi$ especially at low temperatures, $T \ll T_{c}$. This deviation does not essentially affect the response of the junctions on a steady magnetic field (Cassel et al., 2001) and may be taken into account in the circuit design as an additional intrinsic inductance (see Likharev, 1976, 1979; Zubkov et al., 1981; Schüssler and Kümmel, 1993), which has to be added to the geometrical one in the circuit simulation. The shape of the CФR, or more explicitly the energy and phase dependencies of spectral current density, which determine the CФR according to Eq. (2), as well as the phase dependence of the energy distribution function in Eq. (2) become important in an analysis of the dynamic properties of Josephson-junction circuits. In particular, a nonsinusoidal CФR results in the generation of subharmonic Shapiro steps (see, e.g., Cassel et al., 2001), which may lead to instabilities in modes of operation of Josephson voltage standards.

The study of the CФR is also important for understanding the fundamental properties of superconducting materials, such as the symmetry of the superconducting correlation and peculiarities of spin transport in multilayered systems based on superconducting and ferromagnetic materials. As we just saw from the examples described in Sec. VIII, the monitoring of the CФR may provide information that is either impossible to get by any other means, or that can be obtained only by a drastic increase in cooling efforts and careful electromagnetic filtering. CФR measurements are also of fundamental importance as a possible tool for direct detection of time-reversal symmetry breaking at the interfaces of unconventional superconductors.

Modern technology is focused on the fabrication of submicron-size Josephson junctions and will require developments in precise measuring technique. We have seen in this review that the measurement of the CФR permits one to determine experimentally the critical current even in the case of a Josephson energy smaller than that of a thermal fluctuation. Measurements of the C $\Phi R$ may also provide information on the transparency of interfaces, which is important for junction fabrication. These measurements may be used as routine controls in various technological steps and processes.

Finally, the $\varphi$ dependence of a particular Josephsonjunction's energy, which can be directly reconstructed from its experimental C $\Phi \mathrm{R}$, may be important in the design of superconducting quantum bits. Recently, Il'ichev, Wagner, et al. (2002) have applied a method developed for CФR measurements to the characterization of superconducting qubit circuits.

We believe that CФR spectroscopy may become a useful tool for novel fundamental and technological developments.

\section{ACKNOWLEDGMENTS}

We are grateful to our colleagues J. Aarts, M. H. S. Amin, D. Balashov, G. Brammertz, A. Brinkman, I.-M. Buchholz, N. M. Chtchelkatchev, I. A. Devyatov, Ya. V. Fominov, E. Goldobin, D. V. Goncharov, M. Götz, M. Grajcar, R. den Hartog, R. Hlubina, H. E. Hoenig, R. P. J. IJsselstein, Z. Ivanov, W. Kessel, V. M. Krasnov, K. K. Likharev, V. F. Lukichev, H.-G. Meyer, J. Niemeyer, V. A. Oboznov, A. N. Omelyanchouk, N. Oukhanski, A. Peacock, A. Poelaert, H. Rogalla, A. V. Rusanov, V. V. Ryazanov, P. Seidel, M. Siegel, Y. Tanaka, S. K. Tolpygo, J. S. Tsai, F. Wilhelm, J. Yoshida, V. Zakosarenko, A. M. Zagoskin, and A. A. Zubkov for their help and contribution to this work at different stages. Helpful discussions with D. V. Averin, Yu. S. Barash, A. Barone, W. 
Belzig, S. Benacka, A. I. Braginski, C. Bruder, A. I. Buzdin, M. Ebel, M. Feigel'man, M. V. Fistul, H. Hilgenkamp, J. B. Ketterson, V. Khanin, R. Kleiner, P. V. Komissinski, V. P. Koshelets, V. N. Krivoruchko, M. L. Kulić, J. Mannhart, R. Mints, P. Müller, Yu. V. Nazarov, I. P. Nevirkovets, G. A. Ovsyannikov, V. K. Semenov, S. E. Shafranjuk, H.-J. Smilde, O. V. Snigirev, F. Tafuri, S. Uchaikin, A. V. Ustinov, A. F. Volkov, A. D. Zaikin, A. V. Zaitsev, and A. B. Zorin are gratefully acknowledged. The authors acknowledge partial support from D-Wave Systems, Inc. E.I. acknowledges the Deutsche Forschungsgemeinschaft for partial support of this work.

\section{APPENDIX: MICROSCOPIC THEORY OF SUPERCONDUCTIVITY}

We describe below the formalisms that form the basis for description of the stationary Josephson effect in various types of Josephson junctions.

\section{Green's functions}

Quantum field-theoretical methods in terms of Green's functions are a powerful tool in all many-body problems (see, for example, Abrikosov et al., 1975; Rammer and Smith, 1986; Zagoskin, 1998).

The starting point for all problems in superconductivity is the Green's function in Nambu space, which combines the particle and hole space (Nambu, 1960). In a superconductor the Green's function has "anomalous" components, $F\left(x, x^{\prime}\right), F^{\dagger}\left(x, x^{\prime}\right)$, and a normal component $G\left(x, x^{\prime}\right)$, which obey a set of coupled Gor'kov equations (Gor'kov 1958, 1959a, 1959b):

$$
\begin{aligned}
& \left\{i \omega \tau_{3}+\frac{\hbar^{2}}{2 m} \frac{\partial^{2}}{\partial \mathbf{r}_{1}^{2}}+\mu+U\left(\mathbf{r}_{1}\right)+\hat{\Delta}\left(\mathbf{r}_{1}\right)\right\} \hat{G}\left(\mathbf{r}_{1}, \mathbf{r}_{2}\right) \\
& \quad=\delta\left(\mathbf{r}_{1}-\mathbf{r}_{2}\right), \\
& \hat{\Delta}=\left(\begin{array}{cc}
0 & \Delta \\
-\Delta^{*} & 0
\end{array}\right), \quad \hat{G}=\left(\begin{array}{cc}
G & F \\
F^{\dagger} & -G
\end{array}\right) .
\end{aligned}
$$

The pair potential $\Delta$ is determined by the selfconsistency equation

$$
\Delta(\mathbf{r})=-\frac{\lambda}{2} T \sum_{\omega} \operatorname{Tr}\left(\tau_{1}+i \tau_{2}\right) \hat{G}(\mathbf{r}, \mathbf{r}) .
$$

Here $\omega=\pi T(2 n+1)$ are the Matsubara frequency, $\tau_{i}$ are Pauli matrices, $U\left(\mathbf{r}_{1}\right)$ is the electric potential, and $\lambda$ is the strength of the attractive interaction. For a normal metal we have $\lambda=0$ and hence $\Delta=0$, but the pair amplitude $F$ can still be nonzero in various situations (e.g., due to the proximity effect in NS structures, as will be considered in detail below).

The solutions of the Gor'kov equations oscillate as a function of the relative coordinate $\mathbf{r}_{1}-\mathbf{r}_{2}$ on a scale of the Fermi wavelength $\lambda_{\mathrm{F}}$. However, characteristic length scales in the typical problems in superconductivity, $\xi_{0}=\hbar v_{\mathrm{F}} / \Delta$ and $\xi_{T}=\hbar v_{\mathrm{F}} / 2 \pi T$, are much larger than $\lambda_{\mathrm{F}}$. If additionally the characteristic spatial scale of a system exceeds $\lambda_{F}$, it is sufficient to know only the de- pendence of the Green's functions on the center-of-mass coordinate $\left(\mathbf{r}_{1}-\mathbf{r}_{2}\right) / 2$ and integrate out the dependence on the relative coordinate. This procedure leads to the quasiclassical equations of motion for the Green's functions, formulated by Eilenberger (1968) and by Larkin and Ovchinnikov (1968).

\section{The quasiclassical approximation}

\section{a. Eilenberger equations}

We shall now discuss the quasiclassical approximation in equilibrium and stationary situations. The formulation for nonequilibrium and time-dependent problems was introduced by Eliashberg (1971) and Larkin and Ovchinnikov $(1975,1977$; see also the review papers of Schmid, 1981; Larkin and Ovchinnikov, 1986; Rammer and Smith, 1986; Schön, 1986; Lambert and Raimondi, 1998; Belzig et al., 1999).

The derivation of the quasiclassical equations is based on a gradient expansion of the Gor'kov equations using the small parameter $\lambda_{\mathrm{F}} / \xi_{0}$. These equations are written for the functions $f(\mathbf{r}, \mathbf{n})$ and $g(\mathbf{r}, \mathbf{n})$, which are the energy-integrated Gor'kov functions:

$f(\mathbf{r}, \vec{n})=\frac{i}{\pi} \iint F_{p} d \xi, \quad g(\mathbf{r}, \mathbf{n})=\frac{i}{\pi} \iint G_{p} d \xi$.

Here $\xi=p^{2} / 2 m-\mu, \mathbf{r}=\left(\mathbf{r}_{1}+\mathbf{r}_{2}\right) / 2$, and $\vec{n}$ is the unit vector normal to the Fermi surface. The functions $F_{p}$ and $G_{p}$ are defined by the relations

$$
\begin{aligned}
F_{p}= & \frac{1}{(2 \pi)^{3}} \iint F\left(\mathbf{r}_{1}, \mathbf{r}_{2}\right) \\
& \times \exp \left\{i \mathbf{p}\left(\mathbf{r}_{1}-\mathbf{r}_{2}\right) / \hbar\right\} d\left(\mathbf{r}_{1}-\mathbf{r}_{2}\right), \\
G_{p}= & \frac{1}{(2 \pi)^{3}} \iint G\left(\mathbf{r}_{1}, \mathbf{r}_{2}\right) \\
& \times \exp \left\{i \mathbf{p}\left(\mathbf{r}_{1}-\mathbf{r}_{2}\right) / \hbar\right\} d\left(\mathbf{r}_{1}-\mathbf{r}_{2}\right) .
\end{aligned}
$$

The functions $f$ and $g$ satisfy the following normalization and symmetry relations:

$$
\begin{aligned}
& g^{2}(\mathbf{r}, \mathbf{n})+f^{\dagger}(\mathbf{r}, \mathbf{n}) f(\mathbf{r}, \mathbf{n})=1, \\
& f^{\dagger}(\mathbf{r}, \mathbf{n})=f(\mathbf{r},-\mathbf{n}),
\end{aligned}
$$

and obey the Eilenberger equations,

$$
\begin{aligned}
& \mathbf{v}_{F} \partial_{r} \hat{g}+\left[\omega \hat{\tau}_{3}+\hat{\Delta}-\frac{1}{\tau}\langle\hat{g}\rangle, \hat{g}\right]=0, \\
& \Delta \ln \frac{T}{T_{c}}-\pi T \sum_{\omega}\left(\frac{\Delta}{\omega}-\langle f\rangle\right)=0 .
\end{aligned}
$$

Here $\partial_{r}=\nabla-i(2 e / \hbar c) \mathbf{A}$, where $\mathbf{A}$ is a vector potential, $\mathbf{v}_{F}$ is the Fermi velocity, and $\tau$ is the impurity scattering time; square brackets denote commutator, angular brackets denote angle averaging. The matrices $\hat{\Delta}$ and $\hat{g}$ are defined as

$$
\hat{\Delta}=\left(\begin{array}{cc}
0 & \Delta \\
\Delta^{*} & 0
\end{array}\right), \quad \hat{g}=\left(\begin{array}{cc}
g & f \\
f^{\dagger} & -g
\end{array}\right) .
$$


The electrical current density is expressed through $g$ as

$$
J=2 e N(0) \pi T \sum_{\omega}\left\langle\mathbf{v}_{F} \operatorname{Im} g\right\rangle,
$$

where $N(0)$ is the density of states per spin at the Fermi level.

The quasiclassical approximation is not valid in the vicinity of atomically sharp interfaces and potential barriers. Therefore Eq. (A6) should be completed by effective boundary conditions. A derivation of such conditions valid at arbitrary transmission of the interface has been given by Zaitsev (1984) on the basis of the full theory by matching the solutions of the Gor'kov and Eilenberger equations along the classically transmitted and reflected trajectories. For the trajectories connecting two metals, the boundary conditions have the form

$$
\begin{aligned}
& \hat{g}_{1}^{a}=\hat{g}_{2}^{a} \equiv \hat{g}^{a}, \\
& \hat{g}^{a}\left\{R-R\left(\hat{g}^{a}\right)^{2}+D\left(\hat{g}_{-}^{c}\right)^{2}\right\}=D \hat{g}_{-}^{c} \hat{g}_{+}^{c},
\end{aligned}
$$

where $D$ is the transmission probability across the interface, $R=1-D$, and the functions $\hat{g}^{c(a)}$ are defined as

$$
\hat{g}_{ \pm}^{c}=\frac{1}{2}\left(\hat{g}_{1}^{c} \pm \hat{g}_{2}^{c}\right), \quad \hat{g}_{1,2}^{c(a)}=\frac{1}{2}\left\{\hat{g}_{1,2}\left(n_{z}\right) \pm \hat{g}_{1,2}\left(-n_{z}\right)\right\} .
$$

Here $n_{z}$ is a unit vector along the normal to the interface.

For fully reflected trajectories, when the momentum component $p_{\|}$is parallel to the interface and satisfies the condition $p_{F 1}<p_{\|}<p_{F 2}$, the following condition is satisfied:

$$
\hat{g}_{1}^{c}=\hat{g}_{2}^{c} .
$$

A different form of boundary condition, using the socalled Ricatti parametrization (Schopohl and Maki, 1995), was derived by Yip (1997), Eschrig (2000), and Fogelström (2000). This method is more convenient for stable numerical solution of the Eilenberger equations.

\section{b. The dirty limit: Usadel equations}

An important practical case is the superconducting material with strong impurity scattering. If the mean free path is much smaller than the coherence length (quantitative criteria will be given below), in the socalled dirty limit, the Eilenberger equations can be further simplified. In this limit the electron motion is diffusive and the Green's functions are nearly isotropic, i.e., in the main approximation they do not depend on the direction of the vector $\vec{n}$. In this case, expanding the Green's functions in spherical harmonics, it is possible to obtain the equations (see Usadel, 1970) for the isotropic parts $\hat{G}$ of the Green's functions:

$$
i \hbar D \partial_{r}\left[\hat{G}(r) \partial_{r} \hat{G}(r)\right]=\left[\omega \hat{\tau}_{3}+\hat{\Delta}(r), \hat{G}(r)\right],
$$

where

$$
\hat{\Delta}=\left(\begin{array}{cc}
0 & \Delta \\
\Delta^{*} & 0
\end{array}\right), \quad \hat{G}=\left(\begin{array}{cc}
G & F \\
F^{*} & -G
\end{array}\right)
$$

and $D=v_{F} l / 3$ is the diffusion coefficient. The matrix Green's function $\hat{G}$ satisfies the normalization condition

$$
\hat{G}^{2}=1, \quad G^{2}+F F^{*}=1,
$$

and the pair potential $\Delta$ is determined by the selfconsistency equation

$$
\Delta \ln \frac{T_{c}}{T}=\pi T \sum_{\omega}\left(\frac{\Delta}{\omega}-F\right) .
$$

Equations (A11)-(A13) are called in literature the Usadel equations.

The current density in the dirty limit is given by

$$
J(r)=-\frac{i \pi \sigma}{2 e} T \sum_{\omega} \operatorname{Tr}\left[\hat{\tau}_{3} \hat{G}(r) \partial_{r} \hat{G}(r)\right],
$$

where $\sigma=2 e^{2} N(0) D$ is the normal-state conductivity.

In a homogeneous bulk $s$-wave superconductor under equilibrium conditions, the solutions for $G$ and $F$ are

$$
G=\frac{\omega}{\sqrt{\Delta^{2}+\omega^{2}}}, \quad F=\frac{\Delta \exp (i \chi)}{\sqrt{\Delta^{2}+\omega^{2}}},
$$

where $\Delta$ and $\chi$ are the magnitude and phase, respectively, of the pair potential at a given temperature.

The boundary conditions for the Usadel equations at an interface between two superconductors $S_{1,2}$ were derived by Kupriyanov and Lukichev (1988a) as

$$
\begin{aligned}
& p_{F 1}^{2} l_{1} \hat{G}_{1} \frac{d}{d x} \hat{G}_{1}=p_{F 2}^{2} l_{2} \hat{G}_{2} \frac{d}{d x} \hat{G}_{2}, \\
& \gamma_{B} \xi_{1} \hat{G}_{1} \frac{d}{d x} \hat{G}_{1}=\left[\hat{G}_{1}, \hat{G}_{2}\right] .
\end{aligned}
$$

Here $x$ is the coordinate along the normal direction to the interface, $p_{F 1,2}$ and $l_{1,2}$ are the Fermi momenta, and $\xi_{1}$ is the dirty-limit coherence length in $S_{1}$,

$$
\xi_{1}=\sqrt{\frac{\hbar D_{1}}{2 \pi T_{c}}},
$$

where $D_{1}=v_{F 1} l_{1} / 3$ and $T_{c}$ is the transition temperature of $S_{1}$.

The dimensionless parameter $\gamma_{B}$ describes the effect of the interface barrier (Kupriyanov and Lukichev, 1988a),

$$
\gamma_{B}=\frac{2 l_{1}}{3 \xi_{1}}\left\langle\frac{1-D}{x D}\right\rangle=\frac{R_{B}}{\rho_{1} \xi_{1}},
$$

where angular brackets denote averaging over angles of trajectories, $x=p_{F 1 x} / p_{F 1}, D$ is the barrier-transmission coefficient, and $\rho_{1}$ is the normal-state resistivity of a superconductor $S_{1}$.

The boundary conditions (A16) and (A17) have a simple physical interpretation: Eq. (A16) manifests current conservation on both sides of the interface, while Eq. (A17) shows the current continuity across the interface.

According to the definition (A19), $\gamma_{B}=0$ for a fully transparent interface. It follows from Eq. (A17) that the Green's functions $\hat{G}_{1,2}$ are continuous at the interface in 
this case. In the regime of low-barrier transparency (tunnel junction), $\gamma_{B} \gg 1$ and $\hat{G}_{1,2}$ have discontinuities at the interface. Lambert et al. (1997) have shown that the condition (A17) is exact in two limits of high and low barrier transparency, $\gamma_{B}=0$ and $\gamma_{B} \gg 1$, and have also found corrections at the intermediate values of $\gamma_{B} \sim 1$ which, however, do not exceed $10 \%$.

It is useful to introduce another dimensionless parameter describing the proximity effect between $S_{1,2}$, which will be used extensively below. For this purpose we rewrite Eq. (A16) in the form

$$
\gamma \xi_{1} \hat{G}_{1} \partial_{x} \hat{G}_{1}=\xi_{2} \hat{G}_{2} \partial_{x} \hat{G}_{2}
$$

where

$$
\gamma=\frac{\rho_{2} \xi_{2}}{\rho_{1} \xi_{1}} .
$$

The parameter $\gamma$ determines the magnitude of $\hat{G}_{2}$ gradients near the interface: the higher the conductivity in $S_{1}$ compared to that in $S_{2}$, the larger the gradient of $\hat{G}_{2}$ and thus the stronger the deviation of the surface $\hat{G}_{2}$ value compared to the bulk one. In particular, if $S_{1}$ is a weak superconductor (or a normal metal) and $S_{2}$ is a strong superconductor, $\gamma$ determines the strength of suppression of superconductivity in $S_{2}$ near the interface compared to the bulk: no suppression occurs for $\gamma=0$, while strong suppression of $F_{2}$ and $\Delta_{2}$ takes place for $\gamma \gg 1$.

\section{c. $\Phi$ parametrization}

A new function $\Phi$ can be introduced in the following way, which fulfills automatically the normalization condition

$$
G=\frac{\omega}{\sqrt{\omega^{2}+\Phi \Phi^{*}}}, \quad F=\frac{\Phi}{\sqrt{\omega^{2}+\Phi \Phi^{*}}}=\frac{G \Phi}{\omega} .
$$

In the bulk superconductor, $\Phi=\Delta$.

The Usadel equation, the self-consistency equation, and the boundary conditions now take the form

$$
\begin{aligned}
& \Phi=\Delta+\frac{D}{2 \omega} \partial_{x}\left(G^{2} \partial_{x} \Phi\right), \\
& \Delta \ln \frac{T_{c}}{T}=\pi T \sum_{\omega} \frac{\Delta-\Phi G}{\omega}, \\
& \xi_{2} G_{2}^{2} \partial_{x} \Phi_{2}=\gamma \xi_{1} G_{1}^{2} \partial_{x} \Phi_{1}, \\
& \xi_{2} \gamma_{B} G_{2} \partial_{x} \Phi_{2}=G_{1}\left(\Phi_{1}-\Phi_{2}\right) .
\end{aligned}
$$

The current density is given by

$$
J(r)=\frac{\pi \sigma}{e} T \sum_{\omega} \operatorname{Im}\left(G^{2} \Phi^{*} \partial_{x} \Phi\right) .
$$

$\Phi$ parametrization simplifies the analytical treatment in the cases of small gradients (when $\Phi \simeq \Delta$ ) and large gradients [when nongradient terms in Eq. (A23) may be neglected]. Moreover, Eq. (A23) becomes linear when $\Phi$ is small compared to $\omega$.

\section{Ginzburg-Landau equations}

At temperatures near the critical temperature $T_{c}$, a superconductor is described by the Ginzburg-Landau equations,

$$
\xi^{2}(T) \partial_{x}^{2} \Delta+\Delta\left(1-|\Delta|^{2} / \Delta_{0}^{2}\right)=0 .
$$

Here $\Delta_{0}^{2}=\left[8 \pi^{2} / 7 \zeta(3)\right] T_{c}\left(T_{c}-T\right)$, and $\xi(T)$ is the Ginzburg-Landau coherence length, which in the dirty limit is given by

$$
\xi(T)=(\pi / 2) \xi\left(1-T / T_{c}\right)^{-1 / 2},
$$

where $\xi$ is given by Eq. (A18). Equation (A28) can be derived directly from the Usadel equations (A23) and (A24) using expansion of $\Phi$ over small gradients in Eq. (A23) and substituting it into the self-consistency equation (A24).

The supercurrent density in the Ginzburg-Landau theory is given by

$$
J=\frac{\pi}{4 e \rho T_{c} \xi(T)} \operatorname{Im}\left(\Delta^{*} \partial_{x} \Delta\right)
$$

Despite the fact that this theory is limited to temperatures close to $T_{c}$, it has proven very useful in describing current-carrying states in various types of Josephson junctions, due to its computational simplicity compared to the quasiclassical methods. Moreover, the GinzburgLandau method is physically more transparent, since a superconductor is described by a single function $\Delta$ which has the meaning of the superconducting order parameter. It is also commonly referred to as a condensate wave function.

The Ginzburg-Landau equations describe the spatial evolution of $\Delta$ at a length scale of the order of $\xi(T)$. They should be completed at the interfaces by corresponding boundary conditions, knowledge of which is crucial for application of the Ginzburg-Landau theory.

As was first shown by de Gennes $(1964,1966)$, the general form of the boundary condition is

$$
\mathbf{n} \partial_{r} \Delta=\frac{1}{b} \Delta
$$

where $\mathbf{n}$ is the unit vector along the direction normal to the interface and $b$ is the so-called extrapolation length. The quantity $\xi(T) / b$ determines the slope of the order parameter of a superconductor $S$ near the interface, i.e., the spatial scale of a penetration of the order parameter from $S$ into another weaker superconductor or to a normal metal brought into contact with $S$. At the contact with a dielectric or at a free surface of a superconductor, $b=\infty$. For a contact with a normal metal, the parameter $b$ was calculated by de Gennes with accuracy up to numerical prefactor

$$
b=\xi(T) / \gamma,
$$

where $\gamma$ is defined in Eq. (A21). The dependence of $b$ on the parameters of adjacent $S$ and $N$ metals was later studied quantitatively by Ivanov et al. (1978), Barone and Ovchinnikov (1979), and Ivanov et al. (1981). 
4. Critical current in a diffusive point contact: Derivation of the $\mathrm{KO}-1$ result

Kulik and Omelyanchuk (1975a) calculated the supercurrent in a diffusive point contact between two superconductors. Here we reproduce this derivation for the more general case of an asymmetric junction having left and right electrodes with the magnitudes of pair potentials $\Delta_{1}$ and $\Delta_{2}$, respectively. Due to the dirty-limit conditions and the smallness of the contact size, $d \ll \sqrt{\xi_{0} l}$, the supercurrent can be calculated from the Usadel equations (A11)-(A14), in which nongradient terms are neglected.

Let us denote $x$ as the direction normal to the junction plane and put the origin of the $x$ axis in the middle of a ScS structure. We have to find the solutions of the Usadel equations in the constriction region $(-d / 2<x$ $<d / 2$ ) and match them with the bulk solutions in the superconducting electrodes at $x= \pm d / 2$. It is convenient to use $\Phi$ parametrization to solve the problem.

Neglecting nongradient terms in Eq. (A23), we rewrite it in the form

$$
\begin{aligned}
& \frac{1}{\omega} G^{2} \frac{\partial}{\partial x} \Phi^{+}=A, \quad \frac{1}{\omega} G^{2} \frac{\partial}{\partial x} \Phi^{-}=B, \\
& G^{2}=\frac{\omega^{2}}{\omega^{2}+\Phi^{+2}-\Phi^{-2}},
\end{aligned}
$$

where $A$ and $B$ are integration constants and

$$
\Phi^{ \pm}=\frac{1}{2}\left(\Phi \pm \Phi^{*}\right) .
$$

From Eq. (A33) it follows that

$$
B \frac{\partial}{\partial x} \Phi^{+}=A \frac{\partial}{\partial x} \Phi^{-} .
$$

This means that $\Phi^{+}$can be expressed via $\Phi^{-}$as

$$
\Phi^{+}=\frac{A}{B} \Phi^{-}+\omega C=\eta \Phi^{-}+\omega C,
$$

where $C$ is the integration constant. After substitution of Eq. (A36) into Eq. (A33), we get the differential equation for $\Phi^{-}$, and further integration provides

$$
\begin{aligned}
& \frac{\Phi^{-}}{\omega}=\frac{\eta}{1-\eta^{2}} C+\frac{M}{1-\eta^{2}} \tan [M(B x+Q)], \\
& \frac{\Phi^{+}}{\omega}=\frac{1}{1-\eta^{2}} C+\frac{\eta M}{1-\eta^{2}} \tan [M(B x+Q)],
\end{aligned}
$$

where $Q$ is the integration constant, and $M$ $=\sqrt{\left(1-\eta^{2}\right)+C^{2}}$.

Matching these solutions with the Green's functions in the superconducting electrodes at $x= \pm d / 2$, we get the following equations for the integration constants:

$$
\begin{aligned}
& \frac{\Phi_{1,2}^{-}}{\omega}=\frac{\eta}{1-\eta^{2}} C+\frac{M}{1-\eta^{2}} \tan \left[M\left( \pm B \frac{d_{C}}{2}+Q\right)\right], \\
& \frac{\Phi_{1,2}^{+}}{\omega}=\frac{1}{1-\eta^{2}} C+\frac{\eta M}{1-\eta^{2}} \tan \left[M\left( \pm B \frac{d_{C}}{2}+Q\right)\right],
\end{aligned}
$$

where $\Phi_{1,2}^{-}$and $\Phi_{1,2}^{+}$are taken at the left and right sides of the constriction, respectively. The solutions of Eqs. (A39) and (A40) are

$$
\begin{aligned}
& \eta=\frac{\Phi_{2}^{+}-\Phi_{1}^{+}}{\left(\Phi_{2}^{-}-\Phi_{1}^{-}\right)}, \\
& C=\frac{\left(\Phi_{2}^{-} \Phi_{1}^{+}-\Phi_{1}^{-} \Phi_{2}^{+}\right)}{\omega\left(\Phi_{2}^{-}-\Phi_{1}^{-}\right)}, \\
& Q=\frac{1}{2 M}\left[\arctan \frac{\Phi_{2}^{-}-\eta \Phi_{2}^{+}}{\omega M}+\arctan \frac{\Phi_{1}^{-}-\eta \Phi_{1}^{+}}{\omega M}\right], \\
& B=\frac{1}{d M}\left[\arctan \frac{\Phi_{2}^{-}-\eta \Phi_{2}^{+}}{\omega M}-\arctan \frac{\Phi_{1}^{-}-\eta \Phi_{1}^{+}}{\omega M}\right] .
\end{aligned}
$$

Substitution of the solution (A39)-(A44) into the expression for the supercurrent (A27) leads to the formula

$$
\begin{aligned}
I_{S}(\varphi)= & \frac{\pi T}{e R_{N}} \sum_{\omega} \frac{C}{M}\left[\arctan \frac{\Phi_{2}^{-}-\eta \Phi_{2}^{+}}{\omega M}\right. \\
& \left.-\arctan \frac{\Phi_{1}^{-}-\eta \Phi_{1}^{+}}{\omega M}\right]
\end{aligned}
$$

which expresses the supercurrent through the values of the Usadel functions on both sides of the constriction. Here $R_{N}$ is the normal resistance of the junction.

The expression for the supercurrent, Eq. (A45), can be further rewritten in terms of the pair potentials $\Delta_{1}$ and $\Delta_{2}$ (Zubkov et al., 1981),

$$
\begin{aligned}
I_{S}(\varphi)= & \frac{2 \pi T}{e R_{N}} b \sum_{\omega>0} \frac{1}{p}\left\{\arctan \frac{\Delta_{1} d-a b}{p}\right. \\
& \left.+\arctan \frac{\Delta_{2} d+a b}{p}\right\},
\end{aligned}
$$

where

$$
\begin{aligned}
& a=\frac{\Delta_{2}-\Delta_{1}}{\Delta_{2}+\Delta_{1}}, \quad b=\frac{2 \Delta_{1} \Delta_{2}}{\Delta_{1}+\Delta_{2}} \cos (\varphi / 2), \\
& p=\left[b^{2}+\left(a^{2}+1\right) \omega^{2}\right]^{1 / 2}, \quad d=\left(a^{2}+1\right) \sin (\varphi / 2) .
\end{aligned}
$$

In a symmetric junction $\Delta_{1}=\Delta_{2}=\Delta$, the expression for the supercurrent reduces to

$$
\begin{aligned}
I_{S}(\varphi) & =\frac{4 \pi T}{e R_{N}} \sum_{\omega>0}\left[\frac{C}{\sqrt{1+C^{2}}} \arctan \left(\frac{C}{\sqrt{1+C^{2}}} \tan \frac{\varphi}{2}\right)\right] \\
& =\frac{4 \pi T}{e R_{N}} \sum_{\omega>0} \frac{\Delta \cos (\varphi / 2)}{\delta} \arctan \frac{\Delta \sin (\varphi / 2)}{\delta},
\end{aligned}
$$

with $\delta=\sqrt{\Delta^{2} \cos ^{2}(\varphi / 2)+\omega^{2}}$, which coincides with the $\mathrm{KO}-1$ result, Eq. (11).

The remarkable fact is that this result can be reproduced by integration of the supercurrent in a ballistic junction, Eq. (19), over the distribution of transmission eigenvalues $D$ [see Eq. (18)] according to 
$\int_{0}^{1} \rho(D) I(D) d D$, where $\rho(D)$ is the transmission eigenvalue density given by Eq. (18) and $I(D)$ is given by Eq. (19). Straightforward integration for a symmetric junction yields Eq. (A47). The physical reason for the applicability of such a scattering approach is the absence of dephasing of Andreev bound states in a point contact.

\section{REFERENCES}

Abrikosov, A. A., L. P. Gor'kov, and I. E. Dzyaloshinski, 1975, Methods of Quantum Field Theory in Statistical Physics (Dover, New York).

Agrait, N., A. Levy Yeyati, and J. M. van Ruitenbeek, 2003, Phys. Rep. 377, 81.

Aleiner, I. L., H. Clarke, and L. I. Glazman, 1996, Phys. Rev. B 53, R7630.

Amatuni, L. E., V. N. Gubankov, S. A. Kovtonyuk, V. P. Koshelets, G. A. Ovsyannikov, I. L. Serpuchenko, and A. N. Vystavkin, 1987, IEEE Trans. Magn. 23, 680.

Ambegaokar, V., and A. Baratoff, 1963, Phys. Rev. Lett. 10, 486.

Amin, H., M. G. Blamire, and J. E. Evetts, 1992, IEEE Trans. Appl. Supercond. 3, 2204.

Amin, M. H. S., A. N. Omelyanchouk, and A. M. Zagoskin, 2001, Phys. Rev. B 63, 212502.

Aminov, B. A., A. A. Golubov, and M. Yu. Kupriyanov, 1996, Phys. Rev. B 53, 365.

Anderson, P. W., and J. M. Rowell, 1963, Phys. Rev. Lett. 10, 230.

Andreev, A. F., 1964, Zh. Eksp. Teor. Fiz. 46, 1823 [Sov. Phys. JETP 19, 1228 (1964)].

Arnold, G., 1985, J. Low Temp. Phys. 59, 143.

Artemenko, S. N., A. F. Volkov, and A. V. Zaitsev, 1979, Solid

State Commun. 30, 771.

Asano, Y., 2001, Phys. Rev. B 64, 014511; 64, 224515(E).

Aslamazov, L. G., and M. V. Fistul', 1982, Zh. Eksp. Teor. Fiz. 83, 1170 [Sov. Phys. JETP 56, 666 (1982)].

Aslamazov, L. G., and A. I. Larkin, 1969, Pis'ma Zh. Eksp.

Teor. Fiz. 9, 150 [JETP Lett. 9, 87 (1969)].

Aslamazov, L. G., A. I. Larkin, and Yu. N. Ovchinnikov, 1968, Zh. Eksp. Teor. Fiz. 55, 323 [Sov. Phys. JETP 28, 171 (1968)]. Aslamazov, L. G., and S. V. Lempitskii, 1982, Zh. Eksp. Teor. Fiz. 82, 1671 [Sov. Phys. JETP 55, 967 (1982)].

Aslamazov, L. G., and A. F. Volkov, 1986, in Nonequilibrium Superconductivity, edited by D. N. Langenberg and A. I. Larkin (Elsevier, Amsterdam), p. 65.

Averin, D. V., 1998, Solid State Commun. 105, 659.

Averin, D. V., and A. Bardas, 1995, Phys. Rev. Lett. 75, 1834. Averin, D. V., and A. Bardas, 1998, Phys. Rev. B 53, R1705.

Bagwell, P. F., 1992, Phys. Rev. B 46, 12573.

Balashov, D., F.-Im. Buchholz, H. Schulze, M. I. Khabipov, R. Dolata, M. Yu. Kupriyanov, and J. Niemeyer, 2000, Supercond. Sci. Technol. 13, 244.

Balashov, D., F.-Im. Buchholz, H. Schulze, M. I. Khabipov, W. Kessel, and J. Niemeyer, 1998, Supercond. Sci. Technol. 11, 1401.

Balashov, D., M. I. Khabipov, F.-Im. Buchholz, W. Kessel, and J. Niemeyer, 1999, Supercond. Sci. Technol. 12, 864.

Balashov, D., M. Khabipov, F.-I. Buchholz, and J. Niemeyer, 2001, IEEE Trans. Appl. Supercond. 11, 1070.

Barash, Yu. S., 2000, Phys. Rev. B 61, 678.
Barash, Yu. S., and I. V. Bobkova, 2002, Phys. Rev. B 65, 144502.

Barash, Yu. S., I. V. Bobkova, and T. Kopp, 2002, Phys. Rev. B 66, 140503(R).

Barash, Yu. S., H. Burkhardt, and D. Rainer, 1996, Phys. Rev. Lett. 77, 4070.

Barash, Yu. S., A. A. Svidzinsky, and H. Burkhardt, 1997, Phys. Rev. B 55, 15282.

Bardas, A., and D. V. Averin, 1997, Phys. Rev. B 56, R8518.

Bardeen, J., and J. L. Johnson, 1972, Phys. Rev. B 5, 72.

Barone, A., and Yu. N. Ovchinnikov, 1979, Zh. Eksp. Teor. Fiz. 77, 1463 [Sov. Phys. JETP 50, 735 (1979)].

Barone, A., and G. Paternó, 1982, Physics and Application of the Josephson Effect (Wiley, New York).

Baselmans, J. J. A., T. T. Heikkilä, B. J. van Wees, and T. M. Klapwijk, 2002, Phys. Rev. Lett. 89, 207002.

Baselmans, J. J. A., A. F. Morpurgo, B. J. van Wees, and T. M. Klapwijk, 1999, Nature (London) 397, 43.

Baselmans, J. J. A., B. J. van Wees, and T. M. Klapwijk, 2001, Phys. Rev. B 63, 094504.

Baselmans, J. J. A., B. J. van Wees, and T. M. Klapwijk, 2002, Phys. Rev. B 65, 224513.

Baxter, D. V., S. D. Steenwyk, J. Bass, and W. P. Pratt, Jr., 1999, J. Appl. Phys. 85, 4545.

Beenakker, C. W. J., 1991, Phys. Rev. Lett. 67, 3836.

Beenakker, C. W. J., 1997, Rev. Mod. Phys. 69, 731.

Beenakker, C. W. J., and H. van Houten, 1991a, in Solid State Physics, edited by H. Ehrenreich and D. Turnbull (Academic, New York), Vol. 44, p. 1.

Beenakker, C. W. J., and H. van Houten, 1991b, Phys. Rev. Lett. 66, 3056.

Beenakker, C. W. J., and H. van Houten, 1991c, Single Electron Tunneling and Mesoscopic Devices (Springer, Berlin), p. 175.

Behr, R., H. Schulze, F. Müller, J. Kohlmann, and J. Niemeyer, 1999, IEEE Trans. Instrum. Meas. 48, 270.

Belzig, W., F. K. Wilhelm, C. Bruder, G. Schön, and A. D. Zaikin, 1999, Superlattices Microstruct. 25, 1251.

Benz, S. P., 1995, Appl. Phys. Lett. 67, 2714.

Benz, S. P., and C. J. Burroughs, 1997, IEEE Trans. Appl. Supercond. 7, 2434.

Bergeret, F. S., A. F. Volkov, and K. B. Efetov, 2001a, Phys. Rev. B 64, 134506.

Bergeret, F. S., A. F. Volkov, and K. B. Efetov, 2001b, Phys. Rev. Lett. 86, 3140.

Bergeret, F. S., A. F. Volkov, and K. B. Efetov, 2001c, Phys. Rev. Lett. 86, 4096.

Bergeret, F. S., A. F. Volkov, and K. B. Efetov, 2002, Phys. Rev. B 65, 134505.

Blais, A., and A. M. Zagoskin, 2000, Phys. Rev. A 61, 042308.

Blatter, G., V. B. Geshkenbein, and L. B. Ioffe, 2001, Phys. Rev. B 63, 174511.

Blonder, G. E., M. Tinkham, and T. M. Klapwijk, 1982, Phys. Rev. B 25, 4515.

Blum, Y., A. Tsukernik, M. Karpovski, and A. Palevskil, 2002, Phys. Rev. Lett. 89, 187004.

Borisenko, I. V., P. B. Mozhaev, G. A. Ovsyannikov, K. Y. Constantinian, and E. A. Stepantsov, 2002, Physica C 368, 328.

Born, F., D. Cassel, K. Ilin, A. M. Klushin, M. Siegel, A. Brinkman, A. A. Golubov, M. Yu. Kupriyanov, and H. Rogalla, 2003, IEEE Trans. Appl. Supercond. 13, 1079.

Bosco, M. F., A. M. Herr, and M. J. Feldman, 1997, IEEE Trans. Appl. Supercond. 7, 3638.

Böttcher, K., and T. Kopp, 1997, Phys. Rev. B 55, 11670. 
Braginski, A. I., 1992, in Superconducting Devices and their Applications, edited by H. Koch and H. Lubbig (Springer, Berlin), p. 3.

Brammertz, G., A. Poelaert, A. A. Golubov, P. Verhoeve, A. Peacock, and H. Rogalla, 2001, J. Appl. Phys. 90, 355.

Bratus, E. N., V. S. Shumeiko, and G. Wendin, 1995, Phys. Rev. Lett. 74, 2110.

Brinkman, A., D. Cassel, A. A. Golubov, M. Yu. Kupriyanov, M. Siegel, and H. Rogalla, 2001, IEEE Trans. Appl. Supercond. 11, 1146.

Brinkman, A., and A. A. Golubov, 2000, Phys. Rev. B 61, 11297.

Brinkman, A., A. A. Golubov, M. Yu. Kupriyanov, and H. Rogalla, 1999, Supercond. Sci. Technol. 12, 893.

Brinkman, A., A. A. Golubov, H. Rogalla, F. K. Wilhelm, and M. Yu. Kupriyanov, 2003, Phys. Rev. B 68, 224513.

Brouwer, P. W., and C. W. J. Beenakker, 1997, Chaos, Solitons Fractals 8, 1249.

Buchholz, F.-Im., D. Balashov, M. I. Khabipov, D. Hagedorn, R. Dolata, R. Pöpel, and J. Niemeyer, 2001, Physica C 350, 291.

Buchholz, F.-Im., and W. Kessel, 1999, Physica C 326-327, 111. Buitelaar, M. R., W. Belzig, T. Nussbaumer, B. Babi, C. Bruder, and C. Schönenberger, 2003, Phys. Rev. Lett. 91, 057005.

Buitelaar, M. R., T. Nussbaumer, and C. Schönenberger, 2002, Phys. Rev. Lett. 89, 256801.

Bulaevskii, L. N., V. V. Kuzii, and A. A. Sobyanin, 1977, Pis'ma Zh. Eksp. Teor. Phys. 25, 314 [JETP Lett. 25, 290 (1977)].

Buttcher, K., and T. Kopp, 1997, Phys. Rev. B 55, 11670.

Buzdin, A., 2003, Pis'ma, Zh. Eksp. Teor. Phys. 78, 1073 [JETP Lett. 78, 583 (2003)].

Buzdin, A. I., and I. Baladie, 2002, Phys. Rev. B 67, 184519.

Buzdin, A. I., L. N. Bulaevskii, and S. V. Panyukov, 1982, Pis'ma Zh. Eksp. Teor. Phys. 35, 147 [JETP Lett. 35, 178 (1982)].

Buzdin, A. I., and M. Yu. Kupriyanov, 1991, Pis'ma Zh. Eksp. Teor. Phys. 53, 308 [JETP Lett. 53, 321 (1991)].

Buzdin, A. I., B. Vujičic, and M. Yu. Kupriyanov, 1992, Zh. Eksp. Teor. Phys. 101, 231 [Sov. Phys. JETP 74, 124 (1992)]. Capogna, L., and M. G. Blamire, 1996, Phys. Rev. B 53, 5683. Capogna, L., G. Burnell, and M. G. Blamire, 1997, IEEE Trans. Appl. Supercond. 7, 2415.

Cassel, D., G. Pickartz, M. Siegel, E. Goldobin, H. H. Kohlstedt, A. Brinkman, A. A. Golubov, M. Yu. Kupriyanov, and H. Rogalla, 2001, Physica C 350, 276.

Chang, L., and P. F. Bagwell, 1997, Phys. Rev. B 55, 12678.

Chang, V. C. Y., and C. S. Chu, 1997, Phys. Rev. B 55, 6004.

Chrestin, A., R. Kürsten, K. Biedermann, T. Matsuyama, and U. Merkt, 1999, Superlattices Microstruct. 25, 711.

Chrestin, A., T. Matsuyama, and U. Merkt, 1994, Phys. Rev. B 49, 498.

Chrestin, A., T. Matsuyama, and U. Merkt, 1997, Phys. Rev. B 55, 8457.

Chtchelkatchev, N. M., W. Belzig, and C. Bruder, 2002, Pis'ma Zh. Eksp. Teor. Phys. 75, 772 [JETP Lett. 75, 646 (2002)].

Chtchelkatchev, N. M., W. Belzig, Yu. V. Nazarov, and C. Bruder, 2001, Pis'ma Zh. Eksp. Teor. Phys. 74, 357 [JETP Lett. 74, 323 (2001)].

Clarke, J., 1969, Proc. R. Soc. London, Ser. A 308, 447.

Copetti, C. A., F. Rüders, B. Oelze, Ch. Buchal, B. Kabius, and J. W. Seo, 1995, Physica C 253, 63.

Courtois, H., Ph. Gandit, D. Mailly, and B. Pannetier, 1996, Phys. Rev. Lett. 76, 130.
Cuevas, J. C., A. Martin-Rodero, and A. Levy Yeyati, 1996, Phys. Rev. B 54, 7366.

de Bruyn Ouboter, R., and A. N. Omelyanchouk, 1995, Physica B 216, 37.

de Gennes, P. G., 1964, Rev. Mod. Phys. 36, 225.

de Gennes, P. G., 1966, Superconductivity of Metals and Alloys (Benjamin, New York).

Delin, K. A., and A. W. Kleinsasser, 1996, Supercond. Sci. Technol. 9, 227.

Demler, E. A., G. B. Arnold, and M. R. Beasley, 1997, Phys. Rev. B 55, 15174.

den Hartog, R., A. Golubov, P. Verhoeve, A. Poelaert, D. Martin, A. Peacock, A. van Dordrecht, and D. J. Goldie, 2001, Phys. Rev. B 63, 214507.

Deutscher, G., and K. A. Müller, 1987, Phys. Rev. Lett. 59, 1745.

Devyatov, I. A., D. V. Goncharov, and M. Yu. Kupriyanov, 2001a, Zh. Eksp. Teor. Fiz. 119, 749 [Sov. Phys. JETP 92, 652 (2001)].

Devyatov, I. A., D. V. Goncharov, and M. Yu. Kupriyanov, 2001b, Physica C 350, 249.

Devyatov, I. A., D. V. Goncharov, M. Yu. Kupriyanov, and A. A. Golubov, 1995, IEEE Trans. Appl. Supercond. 9, 4300.

Devyatov, I. A., and M. Yu. Kupriyanov, 1993, Zh. Eksp. Teor. Fiz. 104, 3987 [Sov. Phys. JETP 77, 874 (1993)].

Devyatov, I. A., and M. Yu. Kupriyanov, 1994a, Pis'ma Zh. Eksp. Teor. Fiz. 59, 187 [JETP Lett. 59, 200 (1994)].

Devyatov, I. A., and M. Yu. Kupriyanov, 1994b, J. Phys. IV 4, 229.

Devyatov, I. A., and M. Yu. Kupriyanov, 1997a, Pis'ma Zh. Eksp. Teor. Fiz. 65, 159 [JETP Lett. 65, 171 (1997)].

Devyatov, I. A., and M. Yu. Kupriyanov, 1997b, Zh. Eksp.

Teor. Fiz. 112, 342 [Sov. Phys. JETP 85, 189 (1997)].

Devyatov, I. A., and M. Yu. Kupriyanov, 1998, Zh. Eksp. Teor. Fiz. 114, 687 [Sov. Phys. JETP 87, 375 (1998)].

Devyatov, I. A., M. Yu. Kupriyanov, and G. Wendin, 1997, IEEE Trans. Appl. Supercond. 7, 3021.

Dmitriev, P. N., A. B. Ermakov, A. G. Kovalenko, V. P. Koshelets, N. N. Iosad, A. A. Golubov, and M. Yu. Kupriyanov, 1999, IEEE Trans. Appl. Supercond. 9, 3970.

Dobrosavljević-Grujić, L., R. Zikić, and Z. Radović, 2000, Physica C 331, 254.

Dömmel, R., C. Horstmann, M. Siegel, A. I. Braginski, and M. Yu. Kupriyanov, 1995, Appl. Phys. Lett. 67, 1775.

Dorokhov, O. N., 1984, Solid State Commun. 51, 381.

Dresselhaus, P. D., Y. Chong, J. H. Plantenberg, and P. Benz, 2003, IEEE Trans. Appl. Supercond. 13, 930.

Dubos, P., H. Courtois, B. Pannetier, F. K. Wilhelm, A. D. Zaikin, and G. Schön, 2001, Phys. Rev. B 63, 064502.

Eilenberger, G., 1968, Z. Phys. 214, 196.

Eliashberg, G. M., 1971, Zh. Eksp. Teor. Fiz. 61, 1254 [JETP Lett. 34, 668 (1972)].

Eschrig, M., 2000, Phys. Rev. B 61, 9061.

Fogelström, M., 2000, Phys. Rev. B 62, 11812.

Fogelström, M., D. Rainer, and J. A. Sauls, 1997, Phys. Rev. Lett. 79, 281.

Freericks, J. K., B. K. Nikolić, and P. Miller, 2001, Phys. Rev. B 64, 054511.

Freericks, J. K., B. K. Nikolić, and P. Miller, 2002, Int. J. Mod. Phys. B 16, 531.

Freericks, J. K., B. K. Nikolic, and P. Miller, 2003, IEEE Trans. Appl. Supercond. 13, 1089. 
Friedman, J. R., V. Patel, W. Chen, S. K. Tolpygo, and J. E. Lukens, 2000, Nature (London) 406, 43.

Fritzsch, L., H. Elsner, M. Schubert, and H.-G. Meyer, 1999, Supercond. Sci. Technol. 12, 880.

Fritzsch, L., M. Schubert, G. Wende, and H.-G. Meyer, 1998, Appl. Phys. Lett. 73, 1583.

Fulde, P., and A. Ferrell, 1964, Phys. Rev. 135, A550.

Furusaki, A., H. Takayanagi, and M. Tsukada, 1991, Phys. Rev. Lett. 67, 132.

Furusaki, A., H. Takayanagi, and M. Tsukada, 1992, Phys. Rev. B 45, 10563.

Furusaki, A., and M. Tsukada, 1990, Physica B 165\&166B, 967. Furusaki, A., and M. Tsukada, 1991, Phys. Rev. B 43, 10164.

Galaktionov, A. V., and A. D. Zaikin, 2002, Phys. Rev. B 65, 184507.

Gallop, J. C., 1991, SQUIDs, the Josephson Effects and Superconducting Electronics (Hilger, Bristol).

Gao, J., W. A. M. Aarnink, G. J. Gerritsma, and H. Rogalla, 1990, Physica C 171, 126.

Gerritsma, G. J., M. A. J. Verhoeven, R. Moerman, D. H. A. Blank, and H. Rogalla, 1997, J. Alloys Compd. 251, 196.

Geshkenbein, V. B., and A. I. Larkin, 1986, Pis'ma Zh. Eksp. Teor. Fiz. 43, 306 [JETP Lett. 43, 395 (1986)].

Geshkenbein, V. B., A. I. Larkin, and A. Barone, 1987, Phys. Rev. B 36, 235.

Glazman, L. I., and K. A. Matveev, 1988, Zh. Eksp. Teor. Fiz. 94, 332 [JETP 67, 1276 (1989)].

Glazman, L. I., and K. A. Matveev, 1989, Pis'ma Zh. Eksp. Teor. Fiz. 49, 570 [JETP Lett. 49, 659 (1989)].

Gogadze, G. A., and A. M. Kosevich, 1998, Fiz. Nizk. Temp. 24, 716 [Sov. J. Low Temp. Phys. 24, 540 (1998)].

Goldobin, E., D. Koelle, and R. Kleiner, 2002, Phys. Rev. B 66, 100508.

Golub, A., and B. Horovitz, 1994, Phys. Rev. B 49, 4222.

Golubov, A. A., I. V. Devyatov, M. Yu. Kupriyanov, G. J. Gerritsma, and H. Rogalla, 1998, J. Phys. Chem. Solids 59, 2053.

Golubov, A. A., M. A. Gurvitch, M. Yu. Kupriyanov, and S. V. Polonski, 1993, Zh. Eksp. Teor. Fiz. 103, 112 [JETP 76, 915 (1993)].

Golubov, A. A., E. P. Houwman, J. G. Gijsbertsen, V. M. Krasnov, J. Flokstra, H. Rogalla, and M. Yu. Kupriyanov, 1995, Phys. Rev. B 51, 1073.

Golubov, A. A., V. M. Krasnov, and M. Yu. Kupriyanov, 1997, J. Low Temp. Phys. 106, 249.

Golubov, A. A., and M. Yu. Kupriyanov, 1988, J. Low Temp. Phys. 70, 83.

Golubov, A. A., and M. Yu. Kupriyanov, 1989, Zh. Eksp. Teor. Fiz. 96, 1420 [Sov. Phys. JETP 69, 805 (1989)].

Golubov, A. A., and M. Yu. Kupriyanov, 1994, Zh. Eksp. Teor. Fiz. 105, 1442 [JETP 78, 777].

Golubov, A. A., and M. Yu. Kupriyanov, 1998, Pis'ma Zh. Eksp. Teor. Fiz. 67, 478 [JETP Lett. 67, 478 (1998)].

Golubov, A. A., and M. Yu. Kupriyanov, 1999a, Pis'ma Zh. Eksp. Teor. Fiz. 69, 242 [JETP Lett. 69, 262 (1999)].

Golubov, A. A., and M. Yu. Kupriyanov, 1999b, Superlattices Microstruct. 25, 949.

Golubov, A. A., M. Yu. Kupriyanov, and Ya. V. Fominov, 2002a, Pis'ma Zh. Eksp. Teor. Fiz. 75, 223 [JETP Lett. 75, 190 (2002)].

Golubov, A. A., M. Yu. Kupriyanov, and Ya. V. Fominov, 2002b, Pis'ma Zh. Eksp. Teor. Fiz. 75, 588 [JETP Lett. 75, 709 (2002)].
Golubov, A. A., M. Yu. Kupriyanov, and V. F. Lukichev, 1983, Mikroelektronika 12, 342 [Soviet-Microelectronics 12, 180 (1983)].

Golubov, A. A., M. Yu. Kupriyanov, and V. F. Lukichev, 1984, Fiz. Nizk. Temp. 10, 799 [Sov. J. Low Temp. Phys. 10, 418 (1984)].

Golubov, A. A., M. A. J. Verhoeven, I. A. Devyatov, M. Yu. Kupriyanov, G. J. Gerritsma, and H. Rogalla, 1994, Physica C 235-240, 3261.

Gorelik, L. Y., V. S. Shumeiko, R. I. Shekhter, G. Wendin, and M. Jonson, 1995, Phys. Rev. Lett. 75, 1162.

Gor'kov, L. P., 1958, Zh. Eksp. Teor. Fiz. 34, 735 [Sov. Phys. JETP 7, 505 (1958)].

Gor'kov, L. P., 1959a, Zh. Eksp. Teor. Fiz. 361918 [Sov. Phys. JETP 9, 1364 (1959)].

Gor'kov, L. P., 1959b, Zh. Eksp. Teor. Fiz. 37, 833 [Sov. Phys. JETP 10, 593 (1959)].

Götz, M., M. Grajcar, E. Il'ichev, V. V. Khanin, A. B. Zorin, J. Niemeyer, and H.-G. Meyer, 2000, Phys. Rev. B 62, R14 645. Götz, M., V. V. Khanin, H. Schulze, A. B. Zorin, J. Niemeyer, E. Il'ichev, A. Chwala, H. E. Hoenig, and H.-G. Meyer, 2000, Appl. Phys. Lett. 77, 1354.

Grajcar, M., M. Ebel, E. Il'ichev, R. Kürsten, T. Matsuyama, and U. Merkt, 2002, Physica C 372-376, 27.

Grajcar, M., R. Hlubina, E. Il'ichev, and H.-G. Meyer, 2002, Physica C 368, 267.

Gross, R., and B. Mayer, 1991, Physica C 180, 235.

Gubankov, V. N., S. A. Kovtonyuk, V. P. Koshelets, 1985, Zh. Eksp. Teor. Fiz. 89, 1335 [Sov. Phys. JETP 62, 773 (1985)].

Gudkov, A. L., M. Yu. Kupriyanov, and K. K. Likharev, 1988,

Zh. Eksp. Teor. Fiz. 94, 319 [Sov. Phys. JETP 67, 1478 (1988)].

Gunsenheimer, U., U. Schüssler, and R. Kümmel, 1994, Phys.

Rev. B 49, 6111.

Gunsenheimer, U., and A. D. Zaikin, 1994, Phys. Rev. B 50, 6417.

Gurevich, A., and E. A. Pashitskii, 1998, Phys. Rev. B 57, 13878.

Gurvitch, M., W. A. Washington, and H. A. Huggins, 1983, Appl. Phys. Lett. 42, 472.

Haberkorn, W., H. Knauer, and J. Richter, 1978, Phys. Status Solidi A 47, K161.

Hadfield, R. H., G. Burnell, W. E. Booij, S. J. Lloyd, R. W. Moseley, and M. G. Blamire, 2001, IEEE Trans. Appl. Supercond. 11, 1126.

Hadfield, R. H., G. Burnell, Dae Joon Kang, M. G. Blamire, P. Dresselhaus, and S. P. Benz, 2001, Supercond. Sci. Technol. 14, 1086.

Hagedorn, D., R. Dolata, F.-I. Buchholz, and J. Niemeyer, 2002, Physica C 372-376, 7.

Hagedorn, D., R. Dolata, R. Popel, F.-I. Buchholz, and J. Niemeyer, 2001, IEEE Trans. Appl. Supercond. 11, 1134.

Hagedorn, D., M. Khabipov, R. Dolata, F.-I. Buchholz, and J. Niemeyer, 2003, IEEE Trans. Appl. Supercond. 13, 1096.

Halbritter, J., 1993, Phys. Rev. B 48, 9735.

Halbritter, J., 2002, in Advances in Cryogenic Engineering, AIP Conference Proceedings No. 614, edited by Balu Baluchandran, Donald Gubser, and K. Ted Hartwig (AIP, Melville, NY), p. 777.

Hamilton, C. A., S. P. Benz, C. J. Burroughs, and T. E. Harvey, 1997, IEEE Trans. Appl. Supercond. 7, 2472.

Hamilton, C. A., C. J. Burroughs, and R. L. Kauts, 1995, IEEE Trans. Instrum. Meas. 44, 233. 
Haslinger, R., and R. Joynt, 2000, J. Phys.: Condens. Matter 12, 8179.

Heida, J. P., B. J. van Wees, T. M. Klapwijk, and G. Borghs, 1999, Phys. Rev. B 60, 13135.

Heikkilä, T. T., J. Särkkä, and F. K. Wilhelm, 2002, Phys. Rev. B 66, 184513.

Heikkilä, T. T., F. K. Wilhelm, and G. Schön, 2000, Europhys. Lett. 51, 434.

Heslinga, D. R., and T. M. Klapwijk, 1993, Phys. Rev. B 47, 5157.

Hilgenkamp, H., H.-J. Ariando, D. H. A. Smilde, G. Blank, G. Rijnders, H. Rogalla, J. R. Kirtley, and C. C. Tsuei, 2003, Nature (London) 422, 50.

Hilgenkamp, H., and J. Mannhart, 1998, Appl. Phys. Lett. 73, 265.

Hilgenkamp, H., and J. Mannhart, 2002, Rev. Mod. Phys. 74, 485.

Hilgenkamp, H., J. Mannhart, and B. Mayer, 1996, Phys. Rev. B 53, 14586.

Hlubina, R., 2003, in Proceedings of the NATO Advanced Research Workshop Concepts in Electron Correlation, edited by A. C. Hewson and V. Zlatić, NATO Science Series II, Vol. 110 (Kluwer Academic, Dordrecht), p. 17.

Hlubina, R., M. Grajcar, and E. Il'ichev, 2003, in Studies of High Temperature Superconductors, edited by A. Narlikar, Vol. 45, p. 1.

Horstmann, C., P. Leinenbach, R. Dittmann, U. Memmert, U. Hartmann, and A. I. Braginski, 1997, IEEE Trans. Appl. Supercond. 7, 2844.

Horstmann, C., P. Leinenbach, A. Engelhardt, R. Dittmann, U. Memmert, A. I. Braginski, J. L. Jia, R. Gerber, and U. Hartmann, 1998, Physica C 302, 176.

Hu, C. R., 1994, Phys. Rev. Lett. 72, 1526.

Huang, J., F. Pierre, T. T. Heikkilä, F. K. Wilhelm, and N. O. Birge, 2002, Phys. Rev. B 66, 020507.

Huck, A., A. van Otterlo, and M. Sigrist, 1997, Phys. Rev. B 56, 14163.

Il'ichev, E., M. Grajcar, R. Hlubina, R. P. J. IJsselsteijn, H. E. Hoenig, H.-G. Meyer, A. Golubov, M. H. S. Amin, A. M. Zagoskin, A. N. Omelyanchouk, and M. Yu. Kupriyanov, 2001, Phys. Rev. Lett. 86, 5369.

Il'ichev, E., H. E. Hoenig, H.-G. Meyer, A. B. Zorin, V. V. Khanin, M. Götz, A. B. Pavolotsky, and J. Niemeyer, 2001, Physica C 352, 141.

Il'ichev, E., V. Schultze, R. P. J. IJsselsteijn, R. Stolz, V. Zakosarenko, H. E. Hoenig, H.-G. Meyer, and M. Siegel, 2000, Physica C 330, 155.

Il'ichev, E., Th. Wagner, L. Fritzsch, J. Kunert, V. Schultze, T. May, H. E. Hoenig, H.-G. Meyer, M. Grajcar, D. Born, W. Krech, M. Fistul, and A. Zagoskin, 2002, Appl. Phys. Lett. 80, 4184.

Il'ichev, E., V. Zakosarenko, L. Fritzsch, R. Stolz, H. E. Hoenig, H.-G. Meyer, A. B. Zorin, V. V. Khanin, M. Götz, A. B. Pavolotsky, and J. Niemeyer, 2001, Rev. Sci. Instrum. 72, 1882.

Il'ichev, E., V. Zakosarenko, R. P. J. IJsselsteijn, H. E. Hoenig, V. Schultze, H.-G. Meyer, M. Grajcar, and R. Hlubina, 1999a, Phys. Rev. B 60, 3096.

Il'ichev, E., V. Zakosarenko, R. P. J. IJsselsteijn, H. E. Hoenig, H.-G. Meyer, M. V. Fistul, and P. Müller, 1999b, Phys. Rev. B 59, 11502.
Il'ichev, E., V. Zakosarenko, R. P. J. IJsselsteijn, V. Schultze, H.-G. Meyer, H. E. Hoenig, V. N. Glyantsev, and A. Golubov, 1998a, Appl. Phys. Lett. 72, 731.

Il'ichev, E., V. Zakosarenko, R. P. J. IJsselsteijn, V. Schultze, H.-G. Meyer, H. E. Hoenig, H. Hilgenkamp, and J. Mannhart, 1998b, Phys. Rev. Lett. 81, 894.

Il'ichev, E., V. Zakosarenko, R. P. J. IJsselsteijn, V. Schultze, H.-G. Meyer, H. E. Hoenig, and H. Töpfer, 1999c, IEEE Trans. Appl. Supercond. 9, 3994.

Il'ichev, E., V. Zakosarenko, V. Schultze, H. E. Hoenig, H.-G. Meyer, K. O. Subke, H. Burkhard, and M. Schilling, 2000, Appl. Phys. Lett. 76, 100.

Imamura, T., and S. Hasio, 1989, J. Appl. Phys. 66, 2173.

Ioffe, L. B., V. B. Geshkenbein, M. V. Feigelman, A. L. Fauchere, and G. Blatter, 1999, Nature (London) 398, 679.

Iosad, N. N., M. Kroug, T. Zilstra, A. B. Ermakov, B. D. Jackson, M. Zuiddam, F. E. Meijer, and T. M. Klapwijk, 2003, IEEE Trans. Appl. Supercond. 13, 127.

Ishii, C., 1970, Prog. Theor. Phys. 44, 1525.

Ishizaki, M., H. Yamamory, A. Shoji, S. P. Benz, and P. D. Dresselhouse, 2003, IEEE Trans. Appl. Supercond. 13, 1093. Itskovich, I. F., and R. I. Shekhter, 1981, Fiz. Nizk. Temp. 7, 863 [Sov. J. Low Temp. Phys. 7, 418 (1981)].

Ivanov, Z. G., M. Yu. Kupriyanov, K. K. Likharev, S. V. Meriakri, and O. V. Snigirev, 1978, J. Phys. (Paris), Colloq. 39, C6-556.

Ivanov, Z. G., M. Yu. Kupriyanov, K. K. Likharev, S. V. Meriakri, and O. V. Snigirev, 1981, Fiz. Nizk. Temp. 7, 560 [Sov. J. Low Temp. Phys. 7, 274 (1981)].

Ivlev, B. I., and N. B. Kopnin, 1984, Sov. Phys. Usp. 27, 206.

Jackel, L. D., R. A. Buhrman, and W. W. Webb, 1974, Phys. Rev. B 10, 2782.

Jackel, L. D., M. Warlaumont, T. D. Clark, J. C. Brown, R. A. Buhrman, and M. T. Levinsen, 1976, Appl. Phys. Lett. 28, 353.

Jeanneret, B., A. Rufenacht, and C. J. Burroughs, Jr., 2001, IEEE Trans. Instrum. Meas. 50, 188.

Johansson, G., G. Wendin, K. N. Bratus, and V. S. Shumeiko, 1999, Superlattices Microstruct. 25, 905.

Johnson, M. W., B. J. Dalrymple, D. J. Durand, and J. A. Luine, 2003, IEEE Trans. Appl. Supercond. 13, 571.

Josephson, B. D., 1962, Phys. Lett. 1, 251.

Josephson, B. D., 1964, Rev. Mod. Phys. 36, 216.

Josephson, B. D., 1965, Adv. Phys. 14, 419.

Kadigrobov, A., R. I. Shekhter, and M. Jonson, 2001, Europhys. Lett. 54, 394.

Kadin, A. M., 1999, Introducton to Superconducting Circuits (Wiley, New York).

Kashiwaya, S., and Y. Tanaka, 2000, Rep. Prog. Phys. 63, 1641. Kaul, A. B., and T. van Duzer, 2001, IEEE Trans. Appl. Supercond. 11, 88 .

Kaul, A. B., S. R. Whiteley, T. Van Duzer, L. Yu, N. Newman, and J. M. Rowell, 2001, Appl. Phys. Lett. 78, 99.

Khabipov, M. I., D. V. Balashov, F.-Im. Buchholz, W. Kessel, and J. Niemeyer, 1999, IEEE Trans. Appl. Supercond. 9, 4682.

Khabipov, M., D. Balashov, F.-I. Buchholz, and J. Niemeyer, 2002a, Physica C 372-376, 136.

Khabipov, M., D. Balashov, F.-I. Buchholz, and J. Niemeyer, 2002b, IEEE Trans. Appl. Supercond. 11, 1074.

Khlus, V. A., and I. O. Kulik, 1975, Sov. Tech. Phys. Lett. 20, 283. 
Kieler, O., R. Behr, F. Müller, H. Schulze, J. Kohlmann, and J. Niemeyer, 2002, Physica C 372-376, 309.

Klapwijk, T. M., G. E. Blonder, and M. Tinkham, 1982, Physica $\mathrm{B} \& \mathrm{C}$ 109-110, 1657.

Klapwijk, T. M., D. R. Heslinga, and W. M. van Huffelen, 1989, in Superconducting Electronics, edited by H. Weinstock and M. Nisenoff (Springer, Berlin), p. 385.

Kleiner, R., and P. Müller, 1994, Phys. Rev. B 49, 1327.

Kleiner, R., A. S. Katz, A. G. Sun, R. Summer, D. A. Gajewski, S. H. Han, S. I. Woods, E. Dantsker, B. Chen, K. Char, M. B. Maple, R. C. Dynes, and J. Clarke, 1996, Phys. Rev. Lett. 76, 2161.

Kleinsasser, A. W., and W. J. Gallagher, 1989, in Modern Superconducting Devices, edited by D. Rudman and S. Ruggiero (Academic, Boston), p. 325.

Kleinsasser, A. W., R. E. Miller, W. H. Mallison, and G. B. Arnold, 1994, Phys. Rev. Lett. 72, 1738.

Knauer, H., J. Richer, and P. Seidel, 1977, Phys. Status Solidi A 44, 303.

Kohlmann, J., R. Behr, F. Müller, O. Kieler, H. Schulze, and J. Niemeyer, 2002, in Conference Digest. Conference on Precision Electromagnetic Measurements (IEEE, Piscataway, NJ), p. 386.

Kohlmann, J., H. Schulze, R. Behr, F. Müller, and J. Niemeyer, 2001, IEEE Trans. Instrum. Meas. 50, 192.

Komissinski, P. V., E. Il'ichev, G. A. Ovsyannikov, M. Grajcar, R. Hlubina, and Z. Ivanov, 2002, Physica C 368, 271.

Komissinski, P. V., E. Il'ichev, G. A. Ovsyannikov, S. A. Kovtonyuk, M. Grajcar, R. Hlubina, Z. Ivanov, Y. Tanaka, N. Yoshida, and S. Kashiwaya, 2002, Europhys. Lett. 57, 585.

Komissinski, P. V., G. A. Ovsyannikov, E. Il'ichev, and Z. Ivanov, 2001, Piz'ma Zh. Eksp. Teor. Fiz. 73, 405 [JETP Lett. 73, 361 (2001)].

Komissinski, P. V., G. A. Ovsyannikov, Yu. V. Kislinski, I. M. Kotelyanski, and Z. Ivanov, 2002, Zh. Eksp. Teor. Fiz. 122, 1247 [Sov. Phys. JETP 95, 1074 (2002)].

Kontos, T., M. Aprili, J. Lesueur, F. Genêt, B. Stephanidis, and R. Boursier, 2002, Phys. Rev. Lett. 89, 137007.

Kontos, T., M. Aprili, J. Lesueur, and X. Grison, 2001, Phys. Rev. Lett. 86, 304.

Kopnin, N. B., 2001, Theory of Nonequilibrium Superconductivity (Clarendon, Oxford, England).

Kouznetsov, K. A., A. G. Sun, B. Chen, A. S. Katz, S. R. Bahcall, J. Clarke, R. C. Dynes, D. A. Gajewski, S. H. Han, M. B. Maple, J. Giapintzakis, J.-T. Kim, and D. M. Ginsberg, 1997, Phys. Rev. Lett. 79, 3050.

Krasnov, V. M., A. E. Kovalev, A. Yurgens, and D. Winkler, 2001, Phys. Rev. Lett. 86, 2657.

Krasnov, V. M., A. Yurgens, D. Winkler, P. Delsing, and T. Claeson, 2000, Phys. Rev. Lett. 84, 5860.

Krawiec, M., B. L. Gyorffy, and J. F. Annett, 2002, Phys. Rev. B 66, 172505.

Kresin, V. Z., 1986, Phys. Rev. B 34, 7587.

Krivoruchko, V. N., and E. A. Koshina, 2001a, Phys. Rev. B 63, 224515.

Krivoruchko, V. N., and E. A. Koshina, 2001b, Phys. Rev. B 64, 172511.

Krivoruchko, V. N., and E. A. Koshina, 2002, Phys. Rev. B 66, 014521.

Kuhn, D. D., N. M. Chtchelkatchev, G. B. Lesovik, and G. Blatter, 2001, Phys. Rev. B 63, 054520.

Kulić, M. L., and I. M. Kulić, 2001, Phys. Rev. B 63, 104503.
Kulik, I. O., 1969, Zh. Eksp. Teor. Fiz. 57, 1745 [Sov. Phys. JETP 30, 944 (1969)].

Kulik, I. O., and A. N. Omelyanchuk, 1975a, Pis'ma Zh. Eksp. Teor. Fiz. 21, 216 [JETP Lett. 21, 96 (1975)].

Kulik, I. O., and A. N. Omelyanchuk, 1975b, Zh. Eksp. Teor. Fiz. 68, 2139 [Sov. Phys. JETP 41, 1071 (1975)].

Kulik, I. O., and A. N. Omelyanchuk, 1977, Fiz. Nizk. Temp. 3, 945 [Sov. J. Low Temp. Phys. 3, 459 (1977)].

Kulik, I. O., and A. N. Omelyanchuk, 1978, Fiz. Nizk. Temp. 4, 296 [Sov. J. Low Temp. Phys. 4, 142 (1978)].

Kuplevakhskii, S. V., and I. I. Fal'ko, 1990, Pis'ma Zh. Eksp. Teor. Fiz. 52, 957 [JETP Lett. 52, 340 (1990)].

Kuplevakhskii, S. V., and I. I. Fal'ko, 1993, Pis'ma Zh. Eksp. Teor. Fiz. 58, 193 [JETP Lett. 58, 195 (1993)].

Kupriyanov, M. Yu., 1981, Fiz. Nizk. Temp. 7, 700 [Sov. J. Low Temp. Phys. 7, 342 (1981)].

Kupriyanov, M. Yu., 1989, Supercond. Sci. Technol. $2,5$.

Kupriyanov, M. Yu., 1992, Pis'ma Zh. Eksp. Teor. Fiz. 56, 414 [JETP Lett. 56, 399 (1992)].

Kupriyanov, M. Yu., A. Brinkman, A. A. Golubov, M. Siegel, and H. Rogalla, 1999, Physica C 326-327, 16.

Kupriyanov, M. Yu., and K. K. Likharev, 1990, Usp. Fiz. Nauk 160, 49 [Sov. Phys. Usp. 33, 340 (1990)].

Kupriyanov, M. Yu., K. K. Likharev, and V. F. Lukichev, 1981, Physica B \& C 108B, 1001.

Kupriyanov, M. Yu., K. K. Likharev, and V. F. Lukichev, 1982, Zh. Eksp. Teor. Fiz. 83, 431 [Sov. Phys. JETP 56, 235 (1982)]. Kupriyanov, M. Yu., K. K. Likharev, and L. A. Maslova, 1975, in Proceedings of the 14th International Conference on Low Temperature Physics, Otaniemi, Finland (North-Holland, Amsterdam), p. 104.

Kupriyanov, M. Yu., and V. F. Lukichev, 1980, Fiz. Nizk. Temp. 6, 445 [Sov. J. Low Temp. Phys. 6, 210 (1980)].

Kupriyanov, M. Yu., and V. F. Lukichev, 1981, Fiz. Nizk. Temp. 7, 281 [Sov. J. Low Temp. Phys. 7, 137 (1981)].

Kupriyanov, M. Yu., and V. F. Lukichev, 1982, Fiz. Nizk. Temp. 8, 1045 [J. Low Temp. Phys. 8, 526 (1982)].

Kupriyanov, M. Yu., and V. F. Lukichev, 1988a, Zh. Eksp. Teor. Fiz. 94, 139 [Sov. Phys. JETP 67, 1163 (1988)].

Kupriyanov, M. Yu., and V. F. Lukichev, 1988b, Tr. Inst. Obshch. Fiz., Ross. Akad. Nauk 14, 160 [Proceedings of Institute of General Physics Academy of Science USSR (1988)]. Kupriyanov, M. Y., and J. S. Tsai, 1995, IEEE Trans. Appl. Supercond. 5, 2531.

Kurt, H., R. Loloee, K. Eid, W. P. Pratt, Jr., and J. Bass, 2002, Appl. Phys. Lett. 81, 4787.

Kutchinsky, J., R. Taboryski, C. B. Sorensen, J. B. Hansen, and P. E. Lindelof, 1999, Phys. Rev. Lett. 83, 4856.

Kutchinsky, J., R. Taboryski, C. B. Sorensen, J. B. Hansen, and P. E. Lindelof, 2000, Physica B 284-288, 1868.

Lacquaniti, V., S. Gonzini, S. Maggi, E. Monticone, A. Polcari, R. Steni, and D. Andreone, 2000, Int. J. Mod. Phys. B 14, 3044.

Lacquaniti, V., S. Gonzini, S. Maggi, E. Monticone, R. Steni, and D. Andreone, 1999, IEEE Trans. Appl. Supercond. 9, 4245.

Lacquaniti, V., S. Maggi, A. Polcari, R. Steni, and D. Andreone, 2001, IEEE Trans. Appl. Supercond. 11, 1130.

Lacquaniti, V., S. Maggi, R. Steni, C. Cagliero, D. Andreone, and R. Rossi, 2003, IEEE Trans. Appl. Supercond. 13, 146.

Lambert, C. J., and R. Raimondi, 1998, J. Phys.: Condens. Matter 10, 901. 
Lambert, C. J., R. Raimondi, V. Sweeney, and A. F. Volkov, 1997, Phys. Rev. B 55, 6015.

Lapitskaya, I. L., L. V. Filippenko, A. B. Ermakov, S. V. Shitov, G. V. Prokopenko, S. A. Kovtonyuk, and V. P. Koshelets, 2003, IEEE Trans. Appl. Supercond. 13, 107.

Larkin, A. I., and K. A. Matveev, 1987, Zh. Eksp. Teor. Fiz. 93, 1030 [Sov. Phys. JETP 66, 580 (1987)].

Larkin, A. I., and Yu. N. Ovchinnikov, 1964, Zh. Eksp. Teor. Fiz. 51, 1535 [Sov. Phys. JETP 24, 1035 (1964)].

Larkin, A. I., and Yu. N. Ovchinnikov, 1966, Zh. Eksp. Teor. Fiz. 55, 2262 [Sov. Phys. JETP 26, 1200 (1966)].

Larkin, A. I., and Yu. N. Ovchinnikov, 1968, Zh. Eksp. Teor. Fiz. 55, 2262 [Sov. Phys. JETP 26, 1200 (1968)].

Larkin, A. I., and Yu. N. Ovchinnikov, 1975, Zh. Eksp. Teor. Fiz. 68, 1915 [Sov. Phys. JETP 41, 960 (1975)].

Larkin, A. I., and Yu. N. Ovchinnikov, 1977, Zh. Eksp. Teor. Fiz. 73, 299 [Sov. Phys. JETP 46, 155 (1977)].

Larkin, A. I., and Yu. N. Ovchinnikov, 1986, in Nonequilibrium Superconductivity, edited by D. N. Langenberg and A. I. Larkin (Elsevier, Amsterdam), p. 493.

Latyshev, Yu. I., M. B. Gaifullin, T. Yamashita, M. Machida, and Y. Matsuda, 2001, Phys. Rev. Lett. 87, 247007.

Lehnert, K. W., N. Argaman, H.-R. Blank, K. C. Wong, S. J. Allen, E. L. Hu, and H. Kroemer, 1999, Phys. Rev. Lett. 82, 1265.

Lempitskii, S. V., 1983, Zh. Eksp. Teor. Fiz. 85, 1072 [Sov. Phys. JETP 58, 624 (1983)].

Levy Yeyati, A., A. Martin-Rodero, and F. J. Garcia-Vidal, 1995, Phys. Rev. B 51, 3743.

Likharev, K. K., 1976, Pis'ma Zh. Tekhn. Fiz. 2, 29 [Sov. Tech. Phys. Lett. 2, 12 (1976)].

Likharev, K. K., 1979, Rev. Mod. Phys. 51, 101.

Likharev, K. K., 1986, Dynamics of Josephson Junctions and Circuits (Gordon and Breach, New York).

Likharev, K., 1996, Czech. J. Phys. 46, 3331.

Likharev, K., 2000, in Applications of Superconductors, edited by H. Weinstock (Kluwer, Dordrecht), p. 247.

Likharev, K. K., and V. K. Semenov, 1991, IEEE Trans. Appl. Supercond. 1, 3.

Likharev, K. K., and L. A. Yakobson, 1975, Zh. Tekhn. Fiz. 45, 1503 [Sov. Phys. Tech. Phys. 20, 950 (1975)].

Lodder, A., and Yu. V. Nazarov, 2002, Physica C 377, 239.

Löfwander, T., V. S. Shumeiko, and G. Wendin, 2000, Phys. Rev. B 62, R14653.

Löfwander, T., V. S. Shumeiko, and G. Wendin, 2001, Supercond. Sci. Technol. 14, R53.

Ludoph, B., N. van der Post, E. N. Bratus', E. V. Bezuglyi, V. S. Shumeiko, G. Wendin, and J. M. van Ruitenbeek, 2000, Phys. Rev. B 61, 8561.

Maezawa, M., and A. Shoji, 1997, Appl. Phys. Lett. 70, 3603.

Makhlin, Yu., G. Schön, and A. Shnirman, 1999, Nature (London) 398, 305.

Makhlin, Yu., G. Schön, and A. Shnirman, 2001, Rev. Mod. Phys. 73, 357.

Mannhart, J., H. Hilgenkamp, B. Meyer, Ch. Gerber, J. R. Kirtley, K. A. Moler, and M. Sigrist, 1996, Phys. Rev. Lett. 77, 2782.

Marsh, A. M., D. A. Williams, and H. Ahmed, 1994, Phys. Rev. B 50, 8118 .

Martin, A. M., and J. F. Annett, 1998, Phys. Rev. B 57, 8709.

Martin, A. M., and J. F. Annett, 1999, Superlattices Microstruct. 25, 1019.
Martin-Rodero, A., F. J. Garcia-Vidal, and A. Levy Yeyati, 1994, Phys. Rev. Lett. 72, 554.

Martin-Rodero, A., A. Levy Yeyati, and J. C. Cuevas, 1996, Physica B 218, 126.

Martin-Rodero, A., A. Levy Yeyati, and J. C. Cuevas, 1999, Superlattices Microstruct. 25, 925.

Martinis, J. M., S. Nam, J. Aumentado, and C. Urbina, 2002, Phys. Rev. Lett. 89, 117901.

Melsen, J. A., and C. W. J. Beenakker, 1994, Physica B 203, 219.

Melsen, J. A., P. W. Brouwer, K. M. Frahm, and C. W. J. Beenakker, 1996, Europhys. Lett. 35, 7.

Millis, A., D. Rainer, and J. A. Sauls, 1988, Phys. Rev. B 38, 4504.

Mints, R., 1998, Phys. Rev. B 57, R3221.

Mooij, J. E., T. P. Orlando, L. Levitov, L. Tian, C. N. van der Wal, and S. Lloyd, 1999, Science 285, 1036.

Morpurgo, A., B. J. van Wees, and T. M. Klapwijk, 1998, Appl. Phys. Lett. 72, 966.

Muller, C. J., M. C. Koops, B. J. Vleeming, R. de Bruyn Ouboter, and A. N. Omelyanchouk, 1994, Physica C 220, 258.

Mur, L. C., C. J. P. M. Harmans, J. E. Mooij, J. F. Carlin, A.

Rudra, and M. Ilegems, 1996, Phys. Rev. B 54, 2327.

Nakamura, Y., Yu. A. Pashkin, and J. S. Tsai, 1999, Nature (London) 398, 786.

Nambu, Y., 1960, Phys. Rev. 117, 648.

Naveh, Y., V. Patel, D. V. Averin, K. K. Likharev, and J. E. Lukens, 2000, Phys. Rev. Lett. 85, 5404.

Nazarov, Yu. V., 1994a, Phys. Rev. Lett. 73, 134.

Nazarov, Yu. V., 1994b, Phys. Rev. Lett. 73, 1420.

Neurohr, K., Th. Schäpers, J. Malindretos, S. Lachenmann, A. I. Braginski, H. Luth, and A. A. Golubov, 1999, Phys. Rev. B 59, 11197.

Nevirkovets, I. P., 1995, Supercond. Sci. Technol. 8, 575.

Nevirkovets, I. P., 1997, Phys. Rev. B 56, 832.

Nevirkovets, I. P., T. Doderer, A. Laub, M. G. Blamire, and J.

E. Evetts, 1996, J. Appl. Phys. 80, 2321.

Nevirkovets, I. P., and J. B. Ketterson, 2002, Appl. Phys. Lett. 81, 1273.

Nevirkovets, I. P., J. B. Ketterson, and S. Lomatch, 1999, Appl. Phys. Lett. 74, 1624.

Nevirkovets, I. P., J. B. Ketterson, and J. M. Rowell, 2001, J. Appl. Phys. 89, 3980.

Nevirkovets, I. P., J. B. Ketterson, S. E. Shafranjuk, and S. Lomatch, 2000, Phys. Lett. A 269, 238.

Nevirkovets, I. P., J. B. Ketterson, and M. Siegel, 2001, IEEE Trans. Appl. Supercond. 11, 1138.

Nevirkovets, I. P., and S. E. Shafranjuk, 1999, Phys. Rev. B 59, 1311.

Niemeyer, J., 2000, Supercond. Sci. Technol. 13, 546.

Nikolić, B. K., J. K. Freericks, and P. Miller, 2001, Phys. Rev. B 64, 212507.

Nikolić, B. K., J. K. Freericks, and P. Miller, 2002, Phys. Rev. B 65, 064529.

O’Donovan, C., M. D. Lumsden, B. D. Gaulin, and J. P. Carbotte, 1997, Phys. Rev. B 55, 9088.

Ohta, H., T. Matsui, Y. Uchikawa, K. Kobayashi, and M. Aono, 2001, Physica C 352, 186.

Orlando, T. P., and K. A. Delin, 1991, Foundations of Applied Superconductivity (Addison-Wesley, Reading, MA).

Otadoy, R. E. S., and A. Lodder, 2002, Phys. Rev. B 65, 024521. Oukhanski, N., M. Grajcar, E. Il'ichev, and H.-G. Meyer, 2003, Rev. Sci. Instrum. 74, 1145. 
Ovsyannikov, G. A., I. V. Borisenko, and K. I. Konstantinyan, 2000, Vacuum 58, 149.

Ovsyannikov, G. A., I. V. Borisenko, K. I. Konstantinyan, A. D. Mashtakov, and E. A. Stepantsov, 1999, Tech. Phys. Lett. 25, 913.

Ozana, M., A. Shelankov, and J. Tobiska, 2002, Phys. Rev. B 66, 054508.

Pannetier, B., and H. Courtois, 2000, J. Low Temp. Phys. 118, 599.

Park, W., D. V. Baxter, S. Steenwyk, I. Moraru, W. P. Pratt, Jr., and J. Bass, 2000, Phys. Rev. B 62, 1178.

Polushkin, V., S. Uchaikin, S. Knappe, H. Koch, B. David, and D. Grundler, 1995, IEEE Trans. Appl. Supercond. 5, 2790.

Pöpel, R., D. Hagedorn, F.-I. Buchholz, and J. Niemeyer, 2000, in Proceedings of EUCAS 1999, the Fourth European Conference on Applied Superconductivity, edited by X. Obradors, F. Sandiumenge, and J. Fontcuberta (IOP, Bristol, UK), Vol. 2, p. 277.

Pöpel, R., D. Hagedorn, T. Weimann, F.-I. Buchholz, and J. Niemeyer, 2000, Supercond. Sci. Technol. 13, 148.

Procić, V., A. I. Buzdin, and L. Dobrosavljević-Grujić, 1999, Phys. Rev. B 59, 587.

Radović, Z., L. Dobrosavljević-Grujić, and V. Vujićić, 2001, Phys. Rev. B 63, 214512.

Radović, Z., N. Lazarides, and N. Flytzanis, 2003, Phys. Rev. B 68, 014501.

Radović, Z., M. Ledvij, L. Dobrosavljević-Grujić, A. I. Buzdin, and J. R. Clemm, 1991, Phys. Rev. B 44, 759.

Rammer, J., and H. Smith, 1986, Rev. Mod. Phys. 58, 323.

Riedel, R. A., and P. F. Bagwell, 1998, Phys. Rev. B 57, 6084.

Riedel, R. A., L. Chang, and P. F. Bagwell, 1996, Phys. Rev. B 54, 16082.

Rifkin, R., and B. S. Deaver, 1976, Phys. Rev. B 13, 3894.

Rippard, W. H., A. C. Perrella, F. J. Albert, and R. A. Buhrman, 2002, Phys. Rev. Lett. 88, 046805.

Rittenhouse, G. E., and J. M. Graybeal, 1994, Phys. Rev. B 49, 1182.

Rowell, J. M., 1963, Phys. Rev. Lett. 11, 200.

Ryazanov, V. V., V. A. Oboznov, A. Yu. Rusanov, A. V. Veretennikov, A. A. Golubov, and J. Aarts, 2001a, Phys. Rev. Lett. 86, 2427.

Ryazanov, V. V., V. A. Oboznov, A. Yu. Rusanov, A. V. Veretennikov, A. A. Golubov, and J. Aarts, 2001b, Sov. Phys. Usp. 44, 13.

Ryazanov, V. V., V. A. Oboznov, A. V. Veretennikov, and A. Yu. Rusanov, 2002, Phys. Rev. B 65, 020501(R).

Ryazanov, V. V., A. V. Veretennikov, V. A. Oboznov, A. Yu. Rusanov, V. A. Larkin, A. A. Golubov, and J. Aarts, 2000, Physica C 341-348, 1613.

Sachse, H., R. Pöpel, T. Weimann, F. Müller, G. Hein, and J. Niemeyer, 1997, in Proceedings of the Third European Conference on Applied Superconductivity, EUCAS 1997, Eindhoven, edited by H. Rogalla and A. D. H. A. Blank, Institute of Physics Conference Series No. 158 (IOP, Bristol), Vol. 1, p. 555.

Samuelsson, P., A. Ingerman, V. S. Shumeiko, and G. Wendin, 2001, Physica C 352, 82.

Samuelsson, P., J. Lantz, V. S. Shumeiko, and G. Wendin, 2000, Phys. Rev. B 62, 1319.

Samuelsson, P., V. S. Shumeiko, and G. Wendin, 1997, Phys. Rev. B 56, R5763.

Satoh, T., M. Hidaka, M. Yu. Kupriyanov, and J. S. Tsai, 1995, IEEE Trans. Appl. Supercond. 5, 2612.
Satoh, T., M. Hidaka, and S. Tahara, 1998, IEICE Trans. Electron. 81, 1532.

Schäpers, T., 2001, Superconductor/Semiconductor Junctions, Springer Tracts in Modern Physics No. 174 (Springer, Berlin/ Heidelberg).

Schäpers, T., V. A. Guzenko, R. P. Müller, A. A. Golubov, A. Brinkman, G. Crecelius, A. Kaluza, and H. Lüth, 2003, Phys. Rev. B 67, 014522.

Scheer, E., W. Belzig, Y. Naveh, M. H. Devoret, D. Esteve, and C. Urbina, 2001, Phys. Rev. Lett. 86, 284.

Scheer, E., P. Joyes, D. Esteve, C. Urbina, and M. H. Devoret, 1997, Phys. Rev. Lett. 78, 3535.

Schep, K. M., and G. E. W. Bayer, 1997a, Phys. Rev. Lett. 78, 3015.

Schep, K. M., and G. E. W. Bayer, 1997b, Phys. Rev. B 56, 15860.

Schmid, A., 1981, in Nonequilibrium Superconductivity, Phonons, and Kapitza Boundaries, edited by K. E. Gray (Plenum, New York), p. 423.

Schmidt, V. V., 1997, The Physics of Superconductor (Springer, Berlin).

Schön, G., 1986, in Nonequilibrium Superconductivity, edited by D. N. Langenberg and A. I. Larkin (Elsevier, Amsterdam), p. 589.

Schopohl, N., and K. Maki, 1995, Phys. Rev. B 52, 490.

Schubert, M., L. Fritzsch, G. Wende, and H.-G. Meyer, 2001, IEEE Trans. Appl. Supercond. 11, 1066.

Schulze, H., R. Behr, J. Kohlmann, F. Müller, and J. Niemeyer, 2000, Supercond. Sci. Technol. 13, 1293.

Schulze, H., R. Behr, F. Müller, and J. Niemeyer, 1998, Appl. Phys. Lett. 73, 996.

Schüssler, U., and R. Kümmel, 1993, Phys. Rev. B 47, 2754.

SEMATECH International Technology Roadmap for Semiconductors, 2001, http://www.sematech.org/public/index.htm Seviour, R., and A. F. Volkov, 2000, Phys. Rev. B 61, R9273.

Shafranjuk, S. E., I. P. Nevirkovets, and J. B. Ketterson, 2002, Solid State Commun. 121, 457.

Sharvin, Yu. V., 1965, Zh. Eksp. Teor. Fiz. 48, 984 [Sov. Phys. JETP 21, 655 (1965)].

Shaternik, V., M. Belogolovskii, A. Plecenik, S. Benachka, M. Grajcar, and E. Rudenko, 2001, Physica C 350, 187.

Shchelkachev, N. M., 2000, Pis'ma Zh. Eksp. Teor. Fiz. 71, 736 [JETP Lett. 71, 504 (2000)].

Shin, C.-S., Y.-W. Kim, D. Gall, J. E. Greene, and I. Petrov, 2002, Thin Solid Films 402, 172.

Shumeiko, V. S., G. Wendin, and E. N. Bratus', 1993, Phys. Rev. B 48, 13129.

Sigrist, M., 1998, Prog. Theor. Phys. 99, 899.

Sigrist, M., K. Kuboki, P. A. Lee, A. J. Millis, and T. M. Rice, 1996, Phys. Rev. B 53, 2835.

Sigrist, M., and T. M. Rice, 1995, Rev. Mod. Phys. 67, 503.

Sigrist, M., and K. Ueda, 1991, Rev. Mod. Phys. 63, 239.

Silver, A. H., and J. E. Zimmerman, 1967, Phys. Rev. 157, 317.

Smilde, H.-J., H. Hilgenkamp, G. Rijnders, H. Rogalla, and D.

H. Blank, 2002, Appl. Phys. Lett. 80, 4579.

Sols, F., and J. Ferrer, 1994, Phys. Rev. B 49, 15913.

Stampfl, C., and A. J. Freeman, 2003, Phys. Rev. B 67, 064108.

Sugiyama, H., K. Kobata, A. Fujimaki, and H. Hayakawa, 1999, Appl. Supercond. 6, 483.

Sugiyama, H., A. Yanada, M. Ota, A. Fujimaki, and H. Hayakawa, 1997, Jpn. J. Appl. Phys., Part 2 36, L1157.

Sun, A. G., A. Truscott, A. S. Katz, R. C. Dynes, B. W. Veal, and C. Gu, 1996, Phys. Rev. B 54, 6734. 
Sun, J. L., and J. Gao, 2000, Phys. Rev. B 62, 1457.

Sun, Q.-f., J. Wang, and T.-H. Lin, 2000, Phys. Rev. B 62, 648.

Surgers, C., T. Hoss, C. Schönenberger, and C. Strunk, 2002, J.

Magn. Magn. Mater. 240, 598.

Svidzinskii A. V., 1982, Spatially Inhomogeneous Problems in the Theory of Superconductivity (Nauka, Moscow).

Svidzinskii, A. V., T. N. Antsygina, and E. N. Bratus', 1971, Zh.

Eksp. Teor. Fiz. 61, 1612 [Sov. Phys. JETP 3, 860 (1972)].

Takayanagi, H., T. Akazaki, and J. Nitta, 1995a, Phys. Rev. B $\mathbf{5 1}, 1374$.

Takayanagi, H., T. Akazaki, and J. Nitta, 1995b, Phys. Rev. Lett. 75, 3533.

Takayanagi, H., and T. Kawakami, 1985, Phys. Rev. Lett. 54, 2449.

Takeda, M., U. Uzawa, Z. Wang, A. Saito, and A. Kamakami, 2003, IEEE Trans. Appl. Supercond. 13, 692.

Tanaka, Y., 1994, Phys. Rev. Lett. 72, 3871.

Tanaka, Y., and S. Kashiwaya, 1995, Phys. Rev. Lett. 74, 3451. Tanaka, Y., and S. Kashiwaya, 1996, Phys. Rev. B 53, 11957.

Tanaka, Y., and S. Kashiwaya, 1997a, Physica C 274, 357.

Tanaka, Y., and S. Kashiwaya, 1997b, Phys. Rev. B 56, 892.

Tanaka, Y., and S. Kashiwaya, 1999, J. Phys. Soc. Jpn. 68, 3485.

Tanaka, Y., and S. Kashiwaya, 2000, J. Phys. Soc. Jpn. 68, 3485.

Tang, H. X., Z. D. Wang, and Y. Zhang, 1996, Z. Phys. B: Condens. Matter 101, 359.

Tang, H. X., Z. D. Wang, and J. Zhu, 1996, Phys. Rev. B 54, 12509.

Tartakovsky, A. T., and V. M. Fistul', 1988, Zh. Eksp. Teor. Fiz. 94, 353 [Sov. Phys. JETP 67, 1935 (1988)].

Terzioglu, E., and M. R. Beasley, 1998, IEEE Trans. Appl. Supercond. 8, 48.

Tinchev, S. S., 1994, Physica C 222, 173.

Tinkham, M., 1996, Introduction to Superconductivity, 2nd ed. (McGraw-Hill, New York).

Tolga Ilhan, H., H. V. Demir, and P. F. Bagwell, 1998, Phys. Rev. B 58, 15120.

Tolpygo, S. K., A. Brinkman, A. A. Golubov, M. Yu. Kupriyanov, and J. Lukens, 2003, IEEE Trans. Appl. Supercond. 13, 138.

Tolpygo, S. K., E. Cimpoiasu, X. Li, N. Simonian, Yu. A. Polyakov, J. E. Lukens, and K. K. Likharev, 2003, IEEE Trans. Appl. Supercond. 13, 99.

Tsuei, C. C., and J. R. Kirtley, 2000, Rev. Mod. Phys. 72, 969. Usadel, K. D., 1970, Phys. Rev. Lett. 25, 507.

Uzawa, U., M. Takeda, A. Kawakami, and Z. Wang, 2003, IEEE Trans. Appl. Supercond. 13, 688.

van der Post, N., E. T. Peters, I. K. Yanson, and J. M. van Ruitenbeek, 1994, Phys. Rev. Lett. 72, 1738.

van der Wal, C. H., A. C. J. ter Haar, F. K. Wilhelm, R. N. Schouten, C. J. P. M. Harmans, T. P. Orlando, S. Lloyd, and J. E. Mooij, 2000, Science 290, 773.

van Duzer, T., and C. W. Turner, 1981, Principles of Superconducting Devices and Circuits (Elsevier/North-Holland, New York).

van Duzer, T., L. Zheng, X. Meng, C. Loyo, S. R. Whiteley, L. Yu, N. Newman, J. M. Rowell, and N. Yoshikawa, 2002, Physica C 372-376, 1.

van Harlingen, D. J., 1995, Rev. Mod. Phys. 67, 515.

van Huffelen, W. M., T. M. Klapwijk, D. R. Heslinga, M. J. de Boer, and N. van der Post, 1993, Phys. Rev. B 47, 5170.

van Wees, B. J., K.-M. H. Lenssen, and C. J. P. M. Harmans, 1991, Phys. Rev. B 44, 470. van Wees, B. J., H. van Houten, C. W. J. Beenakker, J. G. Willamson, L. P. Kouwenhoven, D. van der Marel, and C. T. Foxon, 1988, Phys. Rev. Lett. 60, 848.

Vengrus, I. I., M. Yu. Kupriyanov, O. V. Snigirev, A. G. Maresov, and S. I. Krasnosvobodtsev, 1994, Pis'ma Zh. Éksp. Teor. Fiz. 59, 372 [JETP Lett. 60, 381 (1994)].

Verbist, K., O. I. Lebedev, G. van Tendeloo, M. A. J. Verhoeven, A. J. H. M. Rijnders, D. H. A. Blank, and H. Rogalla, 1997, Appl. Phys. Lett. 70, 1167.

Verhoeven, M. A. J., G. J. Gerritsma, H. Rogalla, and A. A. Golubov, 1996, Appl. Phys. Lett. 69, 848.

Veretennikov, A. V., V. V. Ryazanov, V. A. Oboznov, A. Yu. Rusanov, V. A. Larkin, and J. Aarts, 2000, Physica B 284-288, 495.

Vion, D., A. Aassime, A. Cottet, P. Joyez, H. Pothier, C. Urbina, D. Esteve, and M. H. Devoret, 2002, Science 296, 886.

Vion, D., P. F. Orfila, P. Joyez, D. Esteve, and M. H. Devoret, 1995, J. Appl. Phys. 77, 2519.

Vleeming, B. J., C. J. Muller, M. C. Koops, and R. de Bruyn Ouboter, 1994, Phys. Rev. B 50, 16741.

Volkov, A. F., 1995, Phys. Rev. Lett. 74, 4730.

Volkov, A. F., F. S. Bergeret, and K. B. Efetov, 2003, Phys. Rev. Lett. 90, 117006.

Volkov, A. F., P. H. C. Magnee, B. J. van Wees, and T. M. Klapwijk, 1995, Physica C 242, 261.

Volkov, A. F., and H. Takayanagi, 1997, Phys. Rev. B 56, 11184.

Volkov, A. F., A. V. Zaitsev, and T. M. Klapwijk, 1993, Physica C 210, 21.

Waldram, J. R., and J. M. Lumley, 1975, Rev. Phys. Appl. 10, 7. Walker, M. B., and J. Lüttmer-Strathmann, 1996, Phys. Rev. B 54, 588 .

Wallraff, A., T. Duty, A. Lukashenko, and A. V. Ustinov, 2003, Phys. Rev. Lett. 90, 037003.

Wang, M.-J., H.-W. Cheng, S.-L. Wu, P.-K. Chuan, and C. C. Chi, 2003, IEEE Trans. Appl. Supercond. 13, 1100.

Wang, Z., A. Saito, A. Kawakami, and H. Hamasaki, 2003, IEEE Trans. Appl. Supercond. 13, 131.

Wen, J. G., N. Koshizuka, C. Traeholt, H. W. Zandbergen, E. M. C. M. Reuvekamp, and H. Rogalla, 1995, Physica C 255, 293.

Wender, G., M. Schubert, T. May, L. Fritzsch, H.-G. Meyer, G. Thummes, Y. Kucukkaplan, L. M. Qiu, J. Kohlmann, J. Niemeyer, H. Hofmeister, and J. Scheerer, 2003, IEEE Trans. Appl. Supercond. 13, 915.

Wendin, G., and V. S. Shumeiko, 1996a, Phys. Rev. B 53, R6006.

Wendin, G., and V. S. Shumeiko, 1996b, Superlattices Microstruct. 20, 569.

Werthamer, N. R., 1966, Phys. Rev. 147, 255.

Whiteley, L., M. Schubert, G. Wende, and H. G. Meyer, 1998, Appl. Phys. Lett. 73, 1583.

Wilhelm, F. K., G. Schön, and A. D. Zaikin, 1998, Phys. Rev. Lett. 81, 1682.

Wilhelm, F. K., G. Schön, and A. D. Zaikin, 2000, Physica B 280, 418.

Willmott, D., 1972, J. Appl. Phys. 43, 4865.

Xu, Y., A. Matsuda, and M. R. Beasley, 1990, Phys. Rev. B 42, 1492.

Yamamori, H., M. Ishizaki, M. Itoh, and A. Shoji, 2002, Appl. Phys. Lett. 80, 1415.

Yanson, I. K., V. M. Svistunov, and I. M. Dmitrenko, 1965, Zh. Éksp. Teor. Fiz. 48, 976 [Sov. Phys. JETP 21, 650 (1965)]. 
Yip, S. K., 1995, Phys. Rev. B 52, 3087.

Yip, S. K., 1997, J. Low Temp. Phys. 109, 547.

Yip, S. K., 1998, Phys. Rev. B 58, 5803.

Yip, S. K., 2000, Phys. Rev. B 62, R6127.

Yoshida, J., 2000, IEICE Trans. Electron. E83, 49.

Yoshida, J., T. Nagano, and T. Hashimoto, 1997, J. Low Temp.

Phys. 106, 327.

Yu, Y., S. Han, X. Chu, S.-I. Chu, and Y. Wang, 2002, Science 296, 889.

Zagoskin, A. M., 1997, J. Phys.: Condens. Matter 9, L419.

Zagoskin, A. M., 1998, Quantum Theory of Many-Body Systems. Technique and Applications (Springer, New York).

Zagoskin, A. M., 2002, Physica C 368, 305.

Zaikin, A. D., 1983, Zh. Eksp. Teor. Fiz. 84, 1560 [Sov. Phys. JETP 57, 910 (1983)].

Zaikin, A. D., 1988, in Nonequilibrium Superconductivity, edited by V. L. Ginzburg (Nova Science, Commack, NY), p. 57.

Zaikin, A. D., and G. F. Zharkov, 1981, Fiz. Nizk. Temp. 7, 375

[Sov. J. Low Temp. Phys. 7, 184 (1981)].

Zaikin, A. D., and G. F. Zharkov, 1981, Zh. Éksp. Teor. Fiz. 81, 1781 [Sov. Phys. JETP 54, 944 (1981)].

Zaikin, A. D., and G. F. Zharkov, 1983, Phys. Lett. 95A, 331.

Zaitsev, A. V., 1976, Fiz. Tverd. Tela 18, 2744 [Sov. Phys. Solid State 18, 1599 (1976)].
Zaitsev, A. V., 1980, Zh. Éksp. Teor. Fiz. 78, 221 [Sov. Phys. JETP 51, 111 (1980)].

Zaitsev, A. V., 1984, Zh. Éksp. Teor. Fiz. 86, 1742 [Sov. Phys. JETP 59, 1015 (1984)].

Zaitsev, A. V., 1990, Pis'ma Zh. Eksp. Teor. Fiz. 51, 35 [JETP Lett. 51, 41 (1990)].

Zaitsev, A. V., 1991, Physica C 185-189, 2539.

Zaitsev, A. V., 1993, Pis'ma Zh. Eksp. Teor. Fiz. 57, 242 [JETP Lett. 57, 253 (1993)].

Zaitsev, A. V., 1995, Pis'ma Zh. Eksp. Teor. Fiz. [JETP Lett. 61, 771 (1995)].

Zaitsev, A. V., and D. V. Averin, 1998, Phys. Rev. Lett. 80, 3602.

Zehnder, A., Ph. Lerch, S. P. Zhao, Th. Nussbaumer, and E. C. Kirk, 1999, Phys. Rev. B 59, 8875.

Zhou, F., P. Charlat, B. Spivak, and B. Pannetier, 1998, J. Low Temp. Phys. 110, 841.

Zhu, Y., W. Li, T.-H. Lin, and Q.-f. Sun, 2002, Phys. Rev. B 66, 134507.

Zubkov, A. A., and M. Yu. Kupriyanov, 1983, Fiz. Nizk. Temp. 9, 548 [Sov. J. Low Temp. Phys. 9, 279 (1983)].

Zubkov, A. A., M. Yu. Kupriyanov, and V. K. Semenov, 1981, Fiz. Nizk. Temp. 7, 1365 [Sov. J. Low Temp. Phys. 7, 661 (1981)]. 